Florida International University FIU Digital Commons

6-11-2018

\title{
Investigating Scale Effects on Analytical Methods of Predicting Peak Wind Loads on Buildings
}

Mohammadtaghi Moravej

Florida International University,mmora229@fiu.edu

DOI: 10.25148 /etd.FIDC006834

Follow this and additional works at: https://digitalcommons.fiu.edu/etd

Part of the Aerodynamics and Fluid Mechanics Commons, Civil Engineering Commons, Computational Engineering Commons, Structural Engineering Commons, and the Structures and Materials Commons

\section{Recommended Citation}

Moravej, Mohammadtaghi, "Investigating Scale Effects on Analytical Methods of Predicting Peak Wind Loads on Buildings" (2018). FIU Electronic Theses and Dissertations. 3799.

https://digitalcommons.fiu.edu/etd/3799 


\section{FLORIDA INTERNATIONAL UNIVERSITY}

Miami, Florida

\section{INVESTIGATING SCALE EFFECTS ON ANALYTICAL METHODS OF PREDICTING PEAK WIND LOADS ON BUILDINGS}

A dissertation submitted in partial fulfillment of the requirements for the degree of DOCTOR OF PHILOSOPHY

in

CIVIL ENGINEERING

by

Mohammadtaghi Moravej 
To: Dean John Volakis

College of Engineering and Computing

This dissertation, written by Mohammadtaghi Moravej, and entitled Investigating Scale Effects on Analytical Methods of Predicting Peak Wind Loads on Buildings, having been approved in respect to style and intellectual content, is referred to you for judgment.

We have read this dissertation and recommend that it be approved.

$\begin{array}{r}\hline \text { Irtishad Ahmad } \\ \hline \text { Seung Jae Lee } \\ \hline \text { Berrin Tansel } \\ \hline \text { Poannis Zisis } \\ \hline \text { Arindam Gan Chowdhury, Co-Major Professor }\end{array}$

Date of Defense: June 11,2018

The dissertation of Mohammadtaghi Moravej is approved.

Dean John Volakis

College of Engineering and Computing

Andrés G. Gil

Vice President for Research and Economic Development and Dean of the University Graduate School

Florida International University, 2018 
(C) Copyright 2018 by Mohammadtaghi Moravej

All rights reserved. 


\section{DEDICATION}

I want to dedicate this dissertation:

To my lovely wife, Hoda, for her love, support, and sacrifice and her invaluable help and encouragement during the last months of my work. This long journey wouldn't be possible without having her by my side.

To my beautiful little son, the light of our lives, Arvin! His birth opened a new window to the beauties of life and to hope for us.

To my dear parents Mrs. Atiyeh Haghdan and Mr. Kazem Moravej, whose wisdom, love and passion for science and nature have nourished my soul throughout the life. I learned my first lessons of commitment and perseverance from them.

To my very kind siblings and precious family - Yasaman, Mahmoud, my little Rasa and Diana, and my in-laws, especially Mr. Abdulreza Rajaei and Mrs. Farzaneh Amirzadeh for their continuous support and encouragement... and to my beloved grandma, uncle and aunt... who passed away while I was far away from home, their kind and heartening words are always with me. 


\section{ACKNOWLEDGMENTS}

I would like to express my sincere gratitude and appreciation to my major advisor Dr. Arindam Chowdhury for his valuable and priceless mentorship, advice and guidance through all the steps of my doctoral studies and research. Besides all what I have learned from him during the course of studies, I have also learned a great deal from his openmindedness, his facilitation of efficient teamwork, and his handling of tough professional situations.

Also, I should express my sincerest gratitude to my co-major advisor, Dr. Peter Irwin, who has always impressed and inspired me with his breadth of knowledge and wisdom. I have been so privileged for having the unique opportunity of working with him. I should thank Dr. Ioannis Zisis for the meticulous reviews that he always has provided to my papers, and for his support and encouragement at different stages of my work. And I wish to give a special thank you to Dr. Emil Simiu whose deep and vast knowledge has always been a huge source of motivation and inspiration for me, and I could rely on his help and advice whenever I encountered a problem.

Furthermore, I would like to thank my dissertation committee members Dr. Berrin Tansel, Dr. Irtishad Ahmad and Dr. Seung Jae Lee for their help, valuable input and accessibility.

It was an advantage for me to be part of an excellent and professional research team at the FIU Wall of Wind facility. Their technical proficiency, knowledge, and expertise made vital contributions toward the successful fulfillment of my experimental work, and I should sincerely thank them all: Walter Conklin, Roy Liu Marques, Raphael Greenbaum, Maryam 
Refan and Ashkan Rasouli. Also, I should thank the undergrad students who helped with the construction of the models.

The financial support provided by FM GLOBAL Company (Project\#WOW-2015-057) and also the National Science Foundation (NSF) Award (NSF Award No. CMMI1151003) under the project of "Full-Scale Simulation of Peak Responses to Reduce Hurricane Damage to Low Buildings and Use of Related Research to Develop HurricaneEngineering Expertise" is much acknowledged and appreciated. Furthermore, I should acknowledge the support from the International Hurricane Research Center (IHRC) at FIU throughout different stages of my work and also the university graduate school for offering me the Dissertation Year Fellowship (DYF) to support the writing stage of my dissertation. 


\title{
ABSTRACT OF THE DISSERTATION \\ INVESTIGATING SCALE EFFECTS ON ANALYTICAL METHODS OF PREDICTING PEAK WIND LOADS ON BUILDINGS
}

\author{
by \\ Mohammadtaghi Moravej \\ Florida International University, 2018 \\ Miami, Florida \\ Professor Arindam Gan Chowdhury, Co-Major Professor \\ Professor Peter Irwin, Co-Major Professor
}

Large-scale testing of low-rise buildings or components of tall buildings is essential as it provides more representative information about the realistic wind effects than the typical small-scale studies, but as the model size increases, relatively less large-scale turbulence in the upcoming flow can be generated. This results in a turbulence power spectrum lacking low-frequency turbulence content. This deficiency is known to have significant effects on the estimated peak wind loads.

To overcome these limitations, the method of Partial Turbulence Simulation (PTS) has been developed recently in the FIU Wall of Wind lab to analytically compensate for the effects of the missing low-frequency content of the spectrum. This method requires posttest analysis procedures and is based on the quasi-steady assumptions. The current study was an effort to enhance that technique by investigating the effect of different model scales and the range of applicability of the method by considering the limitations risen from the underlying theory, and to simplify the 2DPTS (includes both horizontal components of the 
turbulence) by proposing a weighted average method. Assessing the effect of Reynolds number on peak aerodynamic pressures was another objective of the study.

The results from five tested building models show as the model size was increased, PTS results showed a better agreement with the available field data from TTU building. Although for the smaller models (i.e., 1:100,1:50) almost a full range of turbulence spectrum was present, the highest peaks observed at full-scale were not reproduced, which apparently was because of the Reynolds number effect. The most accurate results were obtained when the PTS was used in the case with highest Reynolds number, which was the1:6 scale model with a less than $5 \%$ blockage and a ${ }^{x} \mathrm{Lum}_{\mathrm{um}} / \mathrm{b}_{\mathrm{m}}$ ratio of 0.78 . Besides that, the results showed that the weighted average PTS method can be used in lieu of the 2DPTS approach. So, to achieve the most accurate results, a large-scale test followed by a PTS peak estimation method deemed to be the desirable approach which also allows the ${ }^{\mathrm{x}} \mathrm{L}_{\mathrm{um}} / \mathrm{b}_{\mathrm{m}}$ values much smaller than the ASCE49-12 recommended ratios. 


\section{TABLE OF CONTENTS}

CHAPTER

PAGE

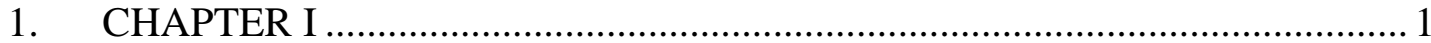

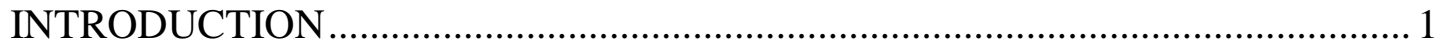

1.1. Wind Tunnel Modeling and Scaling Effects ................................................... 1

1.2. Research Objectives ........................................................................................ 7

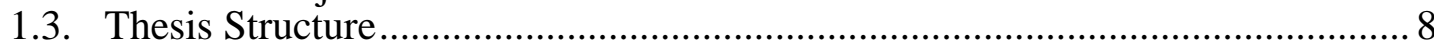

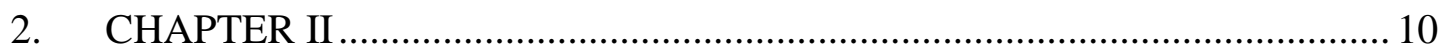

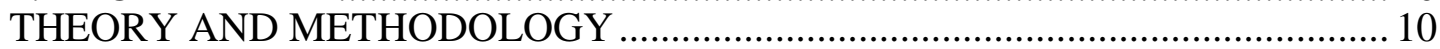

2.1. Introduction to the Partial Turbulence Simulation (PTS) Method ....................... 10

2.2. Challenges in the PTS Method...................................................................... 15

2.3. Proposed Advancements in PTS method ........................................................ 17

2.3.1 Development of the Weighted Average ......................................................... 17

2.3.2 Investigation of Scaling Effects .................................................................. 20

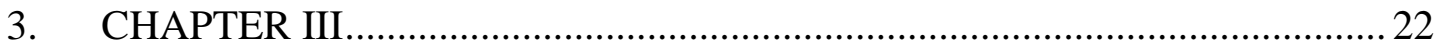

DESIGN OF EXPERIMENTS AND ANALYSIS PROCEDURES ……………..... 22

3.1. Test Buildings, Instrumentation, and Test Protocol ...........................................22

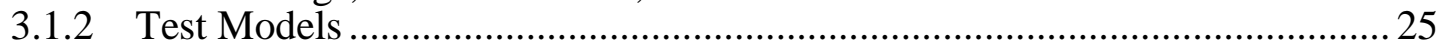

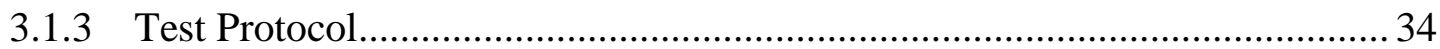

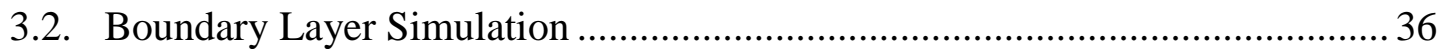

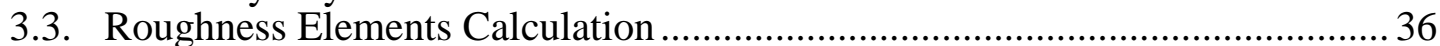

3.4. Wind Speed and Turbulence Intensity Profiles................................................ 40

3.5. Turbulence Power Spectra ……………………………………………….... 43

3.6. Time Scales and Calculation of the PTS Parameters ..........................................45

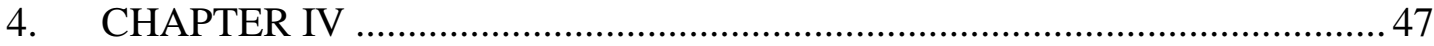

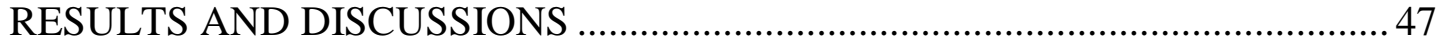

4.1. Comparison of Results of the Weighted Average PTS and the 2DPTS

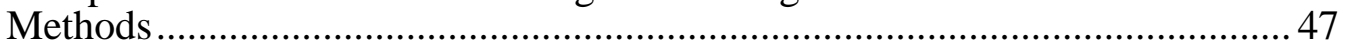

4.2. Comparison of Experimental Results to the Field Data........................................54

4.3. Evaluation of Scaling and Reynolds Number Effects ........................................ 83

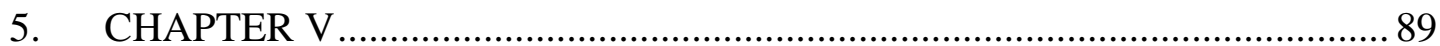

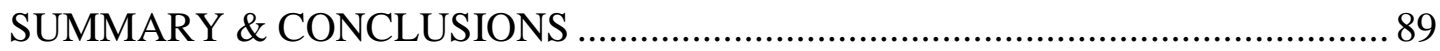

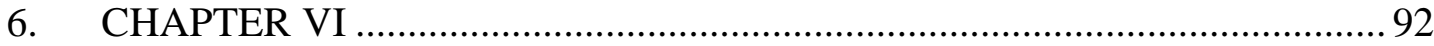

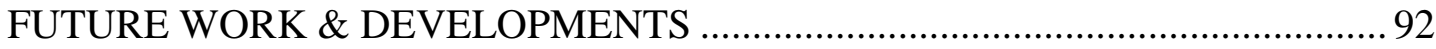

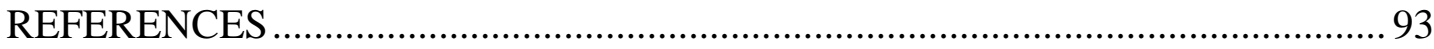

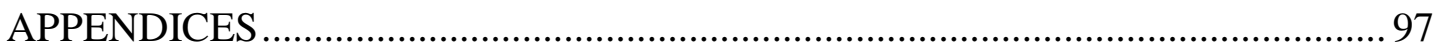

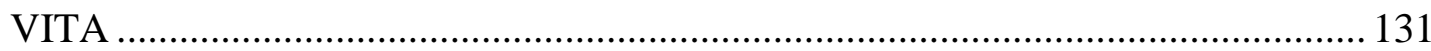




\section{LIST OF TABLES}

TABLE

PAGE

Table 1 - Prototype and model dimensions .......................................................... 25

Table 2 - Roughness element size calculations ........................................................... 37

Table 3 - Measured mean wind speed and turbulence intensity at model eave heights... 41

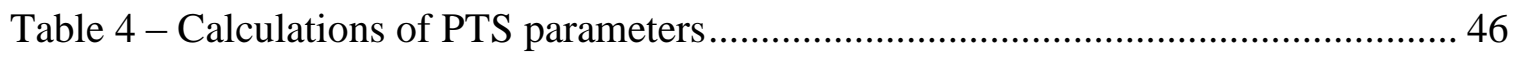

Table 5 - Scaling parameters for the models of building with balcony ....................... 130 


\section{LIST OF FIGURES}

FIGURE

PAGE

Figure 1 - Comparison of Turbulence Spectra between different model scales and fullscale for a tall building of $61 \mathrm{~m}$ height.

Figure 2 - Comparison of Turbulence Spectra between different model scales and fullscale for a low-rise building of $4 \mathrm{~m}$ height.

Figure 3 - Flowchart describing the PTS correction method [51] .....................................

Figure 4 - Numerical solution of the Eq. 14..............................................................14

Figure 5 - Range of Azimuth and tilt angles required for a 3DPTS analysis....................15

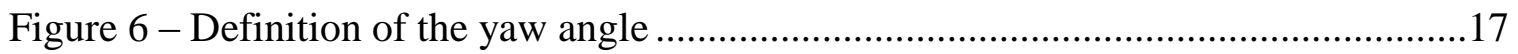

Figure 7 - Distribution of the lateral component of the turbulence.....................................18

Figure 8 - a: Wall of Wind, Florida International University; b: Spires and floor

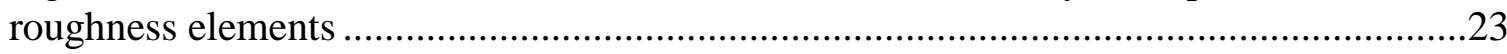

Figure 9 - Schematic drawing of ABL flow generation components for 12-fan WOW ...23

Figure 10 - Conventional notation for the 3 components of wind speed measured by

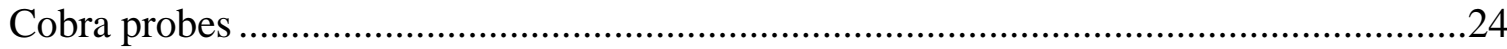

Figure 11 - Schematic of the TTU building model .......................................................26

Figure 12 - TTU field test structure ………………………………………………....27

Figure 13 - Pressure tap installation on the TTU full-scale building .................................27

Figure 14 - Pressure tap layout on the TTU full-scale building .......................................28

Figure 15 - Tap numbering and location map ………………….............................29

Figure $16-1: 100$ scale model built by the stereolithography technique ..........................30

Figure $17-1: 50$ scale model built by the stereolithography technique .............................31

Figure 18 - 1:20 scale model using Plexiglas sheets over wood framing .........................32

Figure 19 - 1:10 scale model using Plexiglas sheets over wood framing ..........................33

Figure 20 - 1:6 scale model using Plexiglas sheets over wood framing .............................34 
Figure 21 - Convention for the wind direction. Side walls are numbered from 1 to 4 while the roof is labeled as side 5 .

Figure 22 - Selected wind angles for Reynolds number tests .....................................35

Figure 23 - Extension of roughness elements for $1: 100$ model........................................38

Figure 24 - Extension of roughness elements for $1: 50$ model.......................................38

Figure 25 - Extension of roughness elements for $1: 20$ model......................................39

Figure 26 - Extension of roughness elements for $1: 10$ model....................................39

Figure 27 - Extension of roughness elements for $1: 6$ model.......................................40

Figure 17 - Normalized Mean wind speed and turbulence intensity profiles ...................42

Figure 29 - Turbulence power spectra ( $\mathrm{n}=$ frequency, $\mathrm{z}=$ model building height, $\mathrm{U}=$ mean wind speed at building eave height, $S=$ turbulence power spectra) ........................44

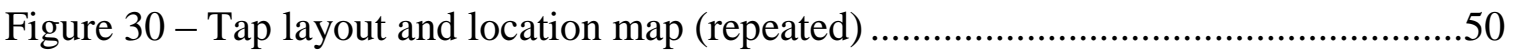

Figure $31-$ Evaluation of the weighted average PTS at roof taps, Dir $=135^{\circ} \ldots \ldots \ldots \ldots \ldots . . . . . . . . . .11$

Figure $32-$ Evaluation of the weighted average PTS at roof taps, Dir $=141^{\circ} \ldots \ldots \ldots \ldots \ldots . . . .51$

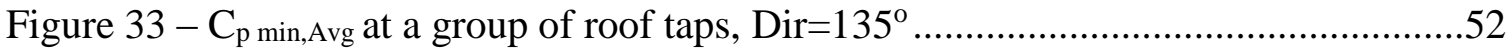

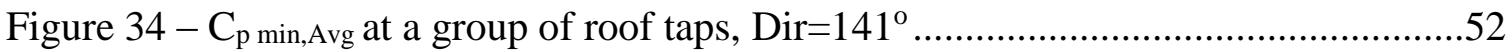

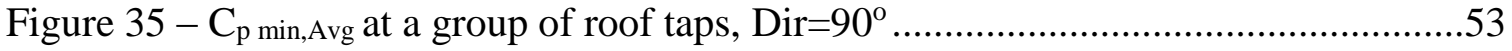

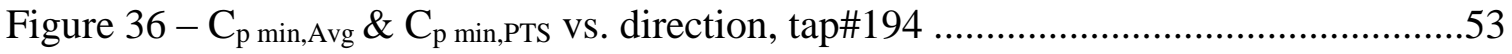

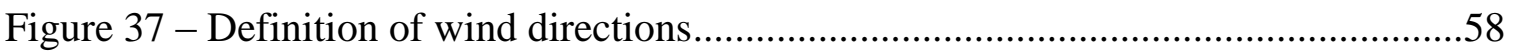

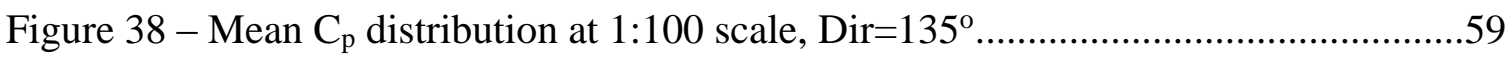

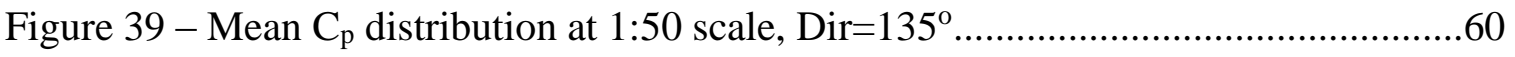

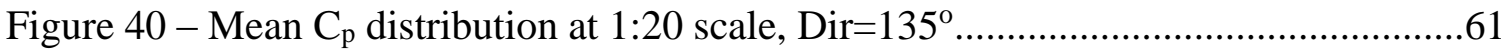

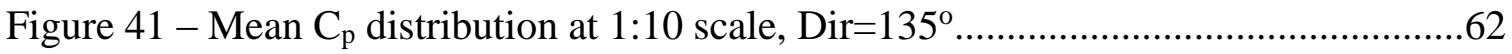

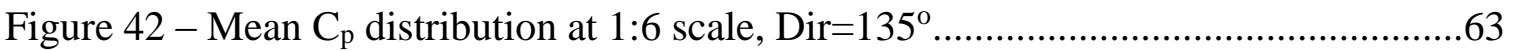

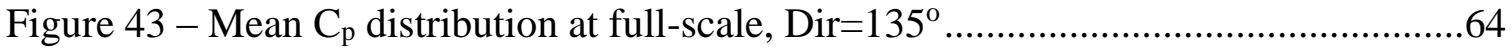


Figure $44-$ Mean $C_{p}$ at scale 1:6, tap-wise display

Figure $45-$ Mean $C_{p}$ at full-scale, tap-wise display ................................................66

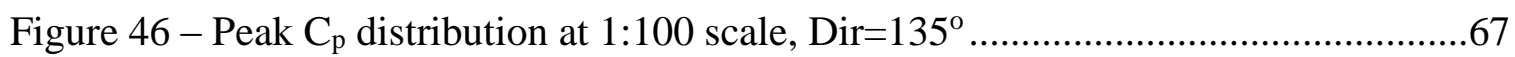

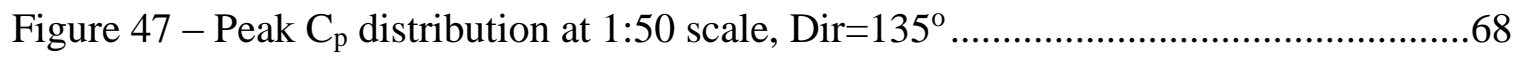

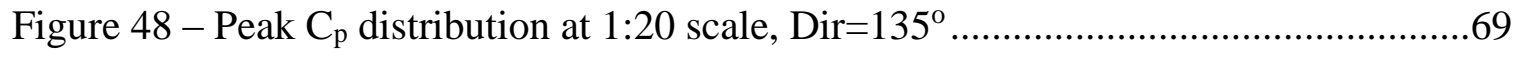

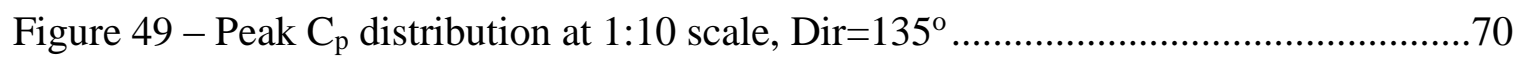

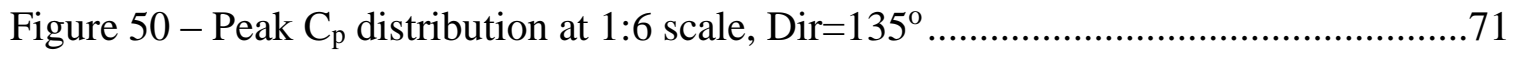

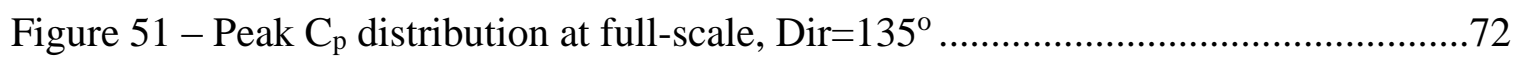

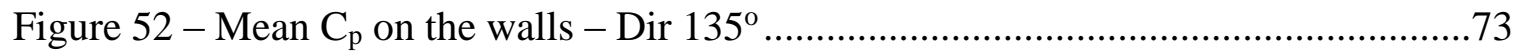

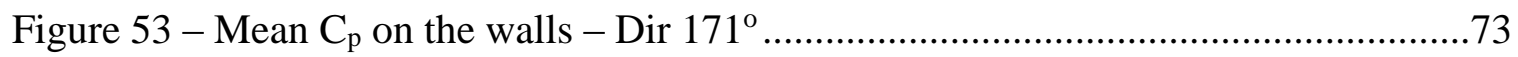

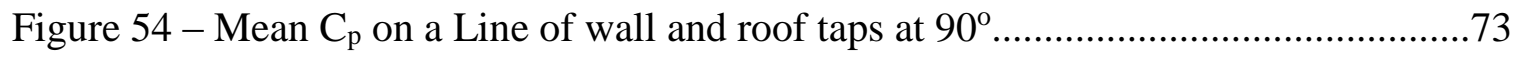

Figure 55 - Wall tap on TTU and a 1:10 model [13] ..............................................74

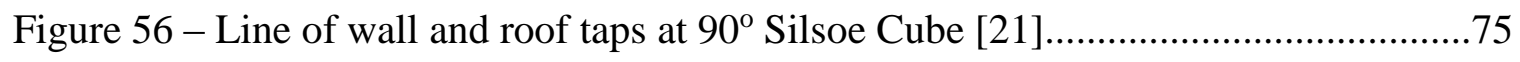

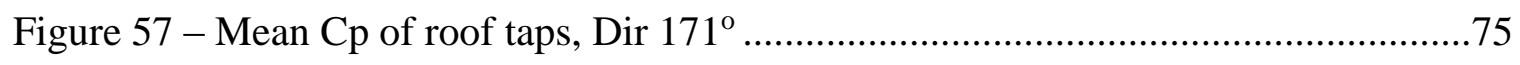

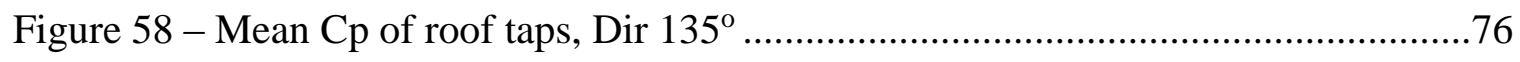

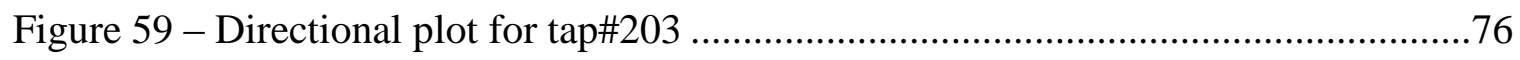

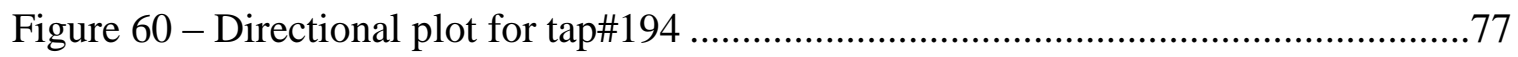

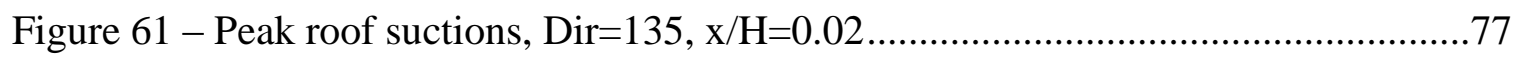

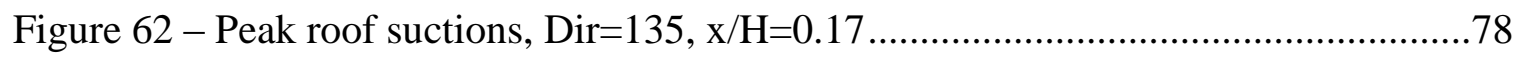

Figure $63-$ Peak roof suctions, Dir=135, y/H=0.04 .......................................... 78

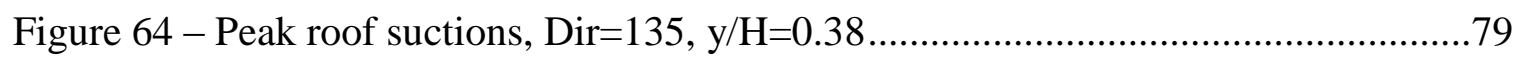

Figure 65 - Peak roof suctions, Dir=144, y/H=0.04 ........................................... 79

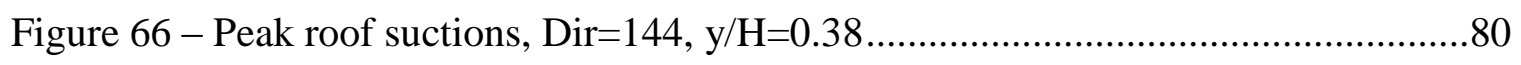

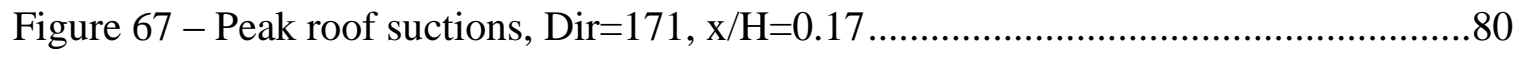




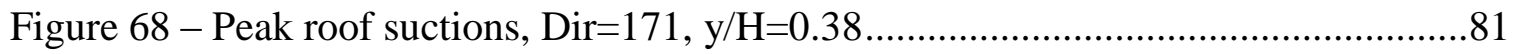

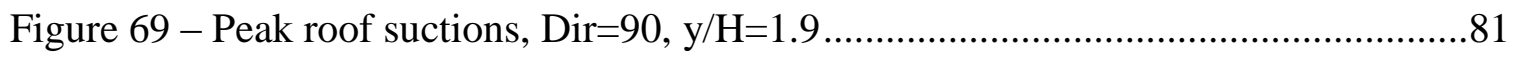

Figure 70 - Minimum $C_{p \text { min,Avg }}$ of all directions (worst case scenario) ..........................82

Figure 71 - Minimum $\mathrm{C}_{\mathrm{p} \text {,min }}$ of all directions (worst case scenario) ............................82

Figure 72 - Comparison between WOW results, TTU (full-scale) \& NIST ....................83

Figure 73 - Observed peaks vs. PTS peaks, roof tap\#194_.......................................86

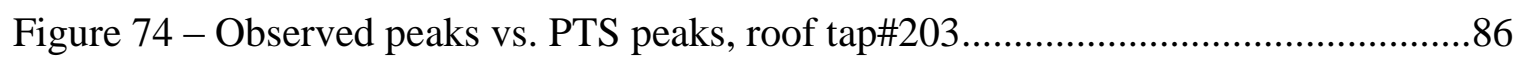

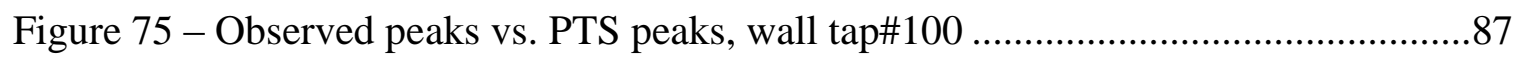

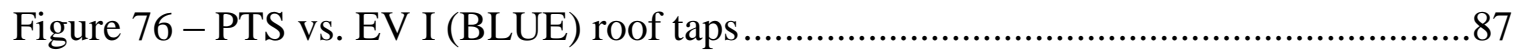

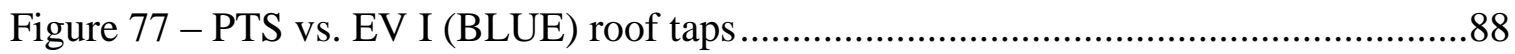

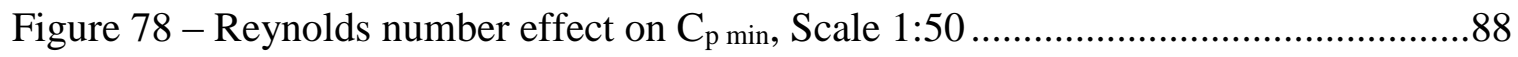

Figure 79 - Reynolds number effect, $\mathrm{C}_{\mathrm{p} \text { min }}$, Scales 1:100 \& 1:6 ..............................88

Figure 80 - Numerical solution of Eq. 14........................................................ 101

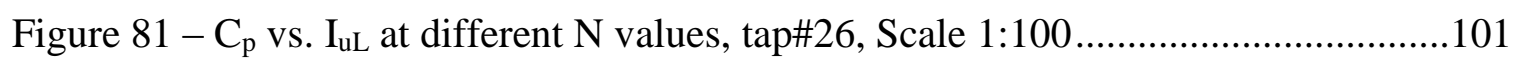

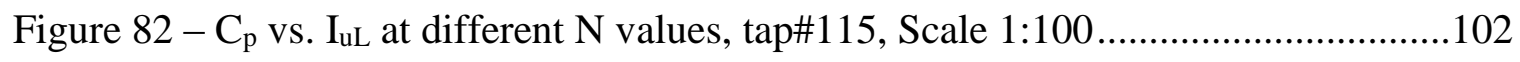

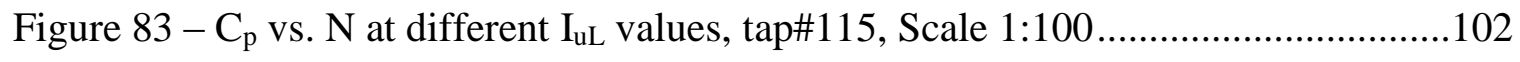

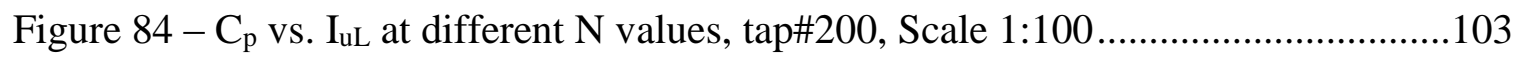

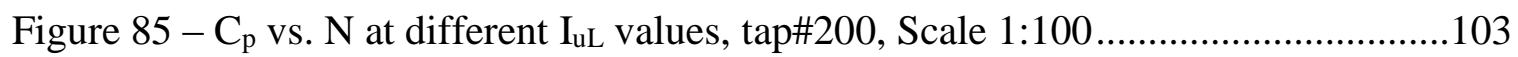

Figure 86 - Excel sheet to calculate PTS analysis basic parameters ...........................104

Figure 87 - MATLAB code for PTS analysis .....................................................106

Figure 88 - MATLAB code for dividing time histories into subintervals ....................107

Figure 89 - MATLAB code for Gumbel fit......................................................... 108

Figure 90 - MATLAB code for the numerical solution of the PTS integral ..................109

Figure 91 - Live MATLAB code to plot wind speed and turbulence spectra ................111 
Figure 92 - MATLAB code to plot model and full-scale turbulence power spectra ......111

Figure 93 - Spectra smoothing code.................................................................112

Figure 94 - MATLAB code for power spectra curve fitting to estimate ${ }^{x} L_{u} \ldots \ldots \ldots \ldots \ldots . . . .112$

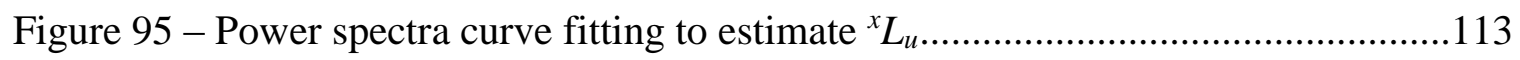

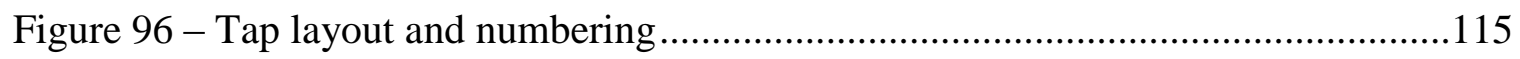

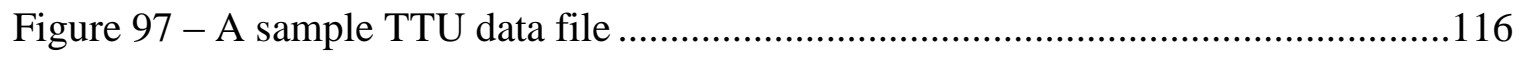

Figure 98 - Tap orders vs. the corresponding column numbers in the data file .............117

Figure 99 - MATLAB code to read TTU data files .....................................................118

Figure 100 - Transfer function setup block diagram.............................................126

Figure 101 - MATLAB program to generate a transfer function from short and long

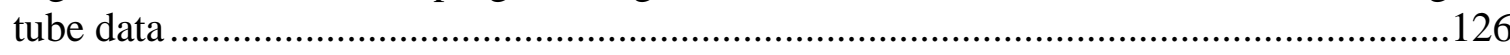

Figure 102 - Transfer function used in WOW (a) vs. TF calculated by Irwin [57] (b) ..127

Figure 103 - Corrected signal versus the long tube and short tube signals ....................128 


\section{CHAPTER I}

\section{INTRODUCTION}

\subsection{Wind Tunnel Modeling and Scaling Effects}

Starting in the mid-twentieth century, wind tunnel testing of structures has become the primary means of establishing wind load provisions for building codes and standards. Also, for large structures, such as tall buildings, stadiums, and long-span bridges, project-specific tests have become the norm as they allow for taking into account the effect of building shape and surrounding terrain and other nearby structures([1]-[6]). Wind tunnel testing of these large structures is typically done on models with scales in the range 1:200 to 1:500. At this range of scales boundary layer wind tunnels can produce an adequate simulation of the turbulent planetary boundary layer, including the correct scaling of the large turbulent eddies and the integral length scales of turbulence.

For smaller structures, such as low-rise buildings, and for building components use of model scales in the range of 1:200 to 1:500 becomes impractical. The models become too small for (i) adequate instrumentation and therefore resolution, (ii) modeling of the finer details that may affect the aerodynamics, and (iii) simulating high enough Reynolds number to avoid scale effects that make the test results no longer fully representative of full-scale. For this reason, larger scale models have been used for low rise buildings, e.g., 1:50 to 1:100 scale, and even at these larger scales Reynolds number effects can sometimes cause deviations from real wind effects measured in the field.

These issues point to the need for larger scale, e.g., 1:5 to 1:30, wind tunnel testing. For evaluating wind effects on and wind resistance of building components, it is even desirable to test at full-scale. For instance, the studies on the wind load reduction factors of vinyl 
wall sidings showed the necessity and importance of a full-scale testing for such building elements [7]-[9]. However, the selection of the model scale for a study is limited by the physical constraints of the wind tunnel and the characteristics of the generated flow. The improper choice of the model scale may have implications on the accuracy of the obtained results.

The effect of scaling on the mean and peak wind pressures and the selection of proper model scale to reproduce accurate results has been the subject of several studies[2], [10][15]. The experimental study by Melbourne [16] indicated that if the model scale exceeds the flow scales, then there will be relatively higher amounts of small-scale turbulence which would lead to overprediction of negative peak pressures under reattachment shear layers. But contrary to that, the experimental study by Hunt [17] concludes that a larger model scale leads to an underestimation of the peak pressures. Stathopoulos and Surry [10] conducted a series of experiments on three models of scales 1:100, 1:250 and 1:500 with not a same upstream surface roughness length. At the 1:500 scale the flow scaling parameters were adequately achieved. They concluded that a small relaxation of turbulence integral length scale, up to a factor of 2 , is permissible for both local and area averaged loads with errors of the order of $10 \%$. Jensen conducted an extensive experimental study on reproducing full scale mean pressure coefficients in wind tunnel and discovered that the ratio of the building height to the roughness height $\left(\mathrm{h} / \mathrm{z}_{0}\right.$, known as the Jensen number) is a significant scaling factor which should be similar between the model and the full scale ( [11], [18]). Wang et al. [19] also concluded that if the roughness height is scaled correctly, then the correct results of the surface pressure coefficients can be obtained even if the 
model dimensions are not scaled correctly. They found that the scale ratio of the roughness height should be the same as the scale ratio of the boundary layer thickness.

To evaluate the accuracy of the wind tunnel modeling and analysis techniques, researchers have tried to compare their findings to the full scale (field) results. The fullscale data from Silsoe Cube ([20]-[24]) and the Texas Tech University (TTU) building ([25]-[30]) have been valuable resources for the comparison purposes and development of the modeling and analysis procedures and also to validate computational models ([15], [31]-[35]). Most of the reported studies have been successful in obtaining mean pressure coefficients close or very similar to those of full scale. But reproducing full-scale peak pressure coefficients has been a challenging task and not completely satisfactory in many cases ([13], [21], [27], [28], [36]-[46]). The difference between the full scale and the scaled model results were found to be more significant at the very corner taps at oblique directions. Lin et al. [47] studied a 1:50 scale model of the TTU building with an improved flow condition with Reynolds numbers ranging from $5 \times 10^{4}$ to $1.5 \times 10^{5}$ and a blockage ratio from $0.3 \%$ to $4 \%$. Their results were generally in good agreement with those of full scale except at the cases and locations with the highest suctions, which as they had observed was at a ray of $14^{\circ}$ from the roof corner at the wind attack angles of $30^{\circ}$ or $60^{\circ}$. Cheung et al. [13] studied a 1:10 scale model of the TTU building, the result of which showed a better agreement with full-scale data comparing to the similar previous works. The highest magnitude of the pressure coefficients they obtained was $20 \%$ less than the corresponding full-scale value, which still marked an improvement over other studies using smaller scale models. They attributed this enhanced agreement mainly to the high Reynolds number of the tests since they had tried to eliminate other factors which could influentially affect the 
results. For instance, even the pressure taps diameter was appropriately scaled. They also concluded that the only way to approach the large peak pressures of full-scale building is to increase the turbulence intensity of the flow above the full-scale values. Lin et al. [47] discussed that the discrepencies between the model and full-scale results can be the result of several factors that can be categorized into three classes: 1) the difference in experimental conditions, flow characteristics, tap size, geometric details, and also the uncertainties of field measurements like the natural variability of the wind parameters, low speed of the wind (increasing effect of convective turbulence), etc. 2) definition of parameters, for example, the characteristic length used for normalization, 3) Reynolds number violation and scaling problems.

The problem with going to the large model scales, which already exists to some extent even at scales of 1:50 to 1:100, is that there are difficulties in simulating the full wind turbulence spectrum, and this can affect the pressure distributions on the model ([1], [10], [14], [16]). The size of the wind tunnel working section limits the size of the largest turbulent eddies that can be simulated, and the end result is that the low-frequency end of the turbulence spectrum is not simulated adequately. The effects of these large-scale turbulent eddies are therefore not fully accounted for within the tests. Figure 1 and Figure 2 illustrate how the simulated turbulence power spectrum has a progressively larger deficit at low frequencies as the model scale is increased. The spectra are normalized using the mean velocity and model height which are turbulence-independent parameters, so the differences in the spectra are not masked ([1], [14], [17]). The high-frequency end of the turbulence spectra matches well, thus ensuring correct aerodynamics and realistic pressure distributions. But the values of the mean and observed peak pressures, as well as the peak 
to mean dynamic pressure ratios are significantly affected by the reduced turbulence intensity due to missing low-frequency turbulence for large models (such as for 1:50 and 1:100 scales) [20], [21].

To overcome these limitations and allow meaningful results to be obtained from tests on larger scale models, and even full-scale building components, the research group at the Wall of Wind (WOW) at Florida International University (FIU) has developed a Partial Turbulence Simulation (PTS) method ([48], [49]) as conceptually illustrated in Figure 3. In this method, the power in the turbulence spectrum at high frequencies is accurately simulated in the WOW, and the effects of the missing low-frequency part of the spectrum are accounted for analytically in the post-test analysis using quasi-steady assumptions. The high-frequency part of the spectrum mainly affects the details of flow separation and reattachment around a body whereas the low-frequency component can be approximately treated similarly to changes in the mean flow velocity. To study the effectiveness of the PTS method, full-scale data obtained from the TTU experimental building [50] and the Silsoe Cube [20], [21] was used by Asghari [48] and Asghari et al. [49] as benchmarks. The application of the PTS method showed good agreement between model test results for scales as large as 1:5 and field data. However, the study had limitations regarding the scales, number of taps, and the modeling of lateral turbulence which are discussed in more detail in the next section. 


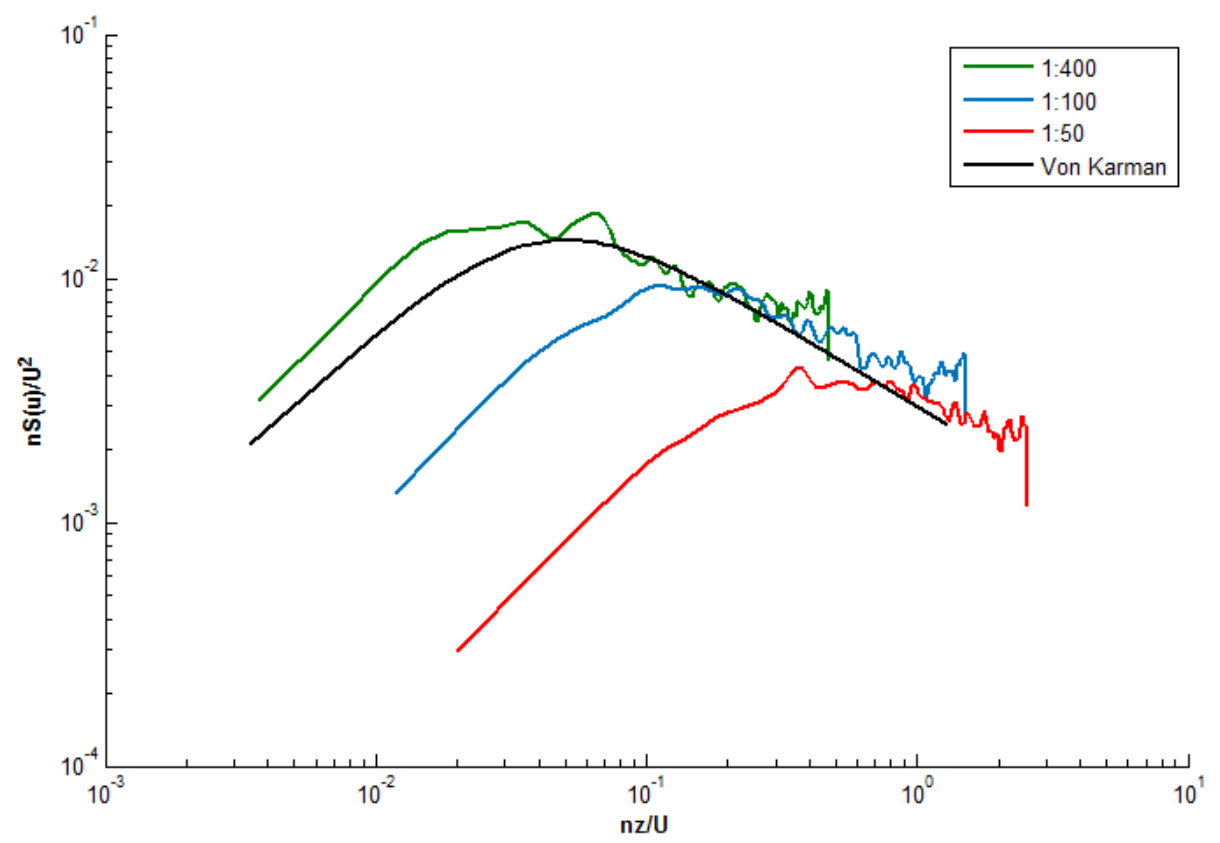

Figure 1 - Comparison of Turbulence Spectra between different model scales and full-scale for a tall building of $61 \mathrm{~m}$ height.

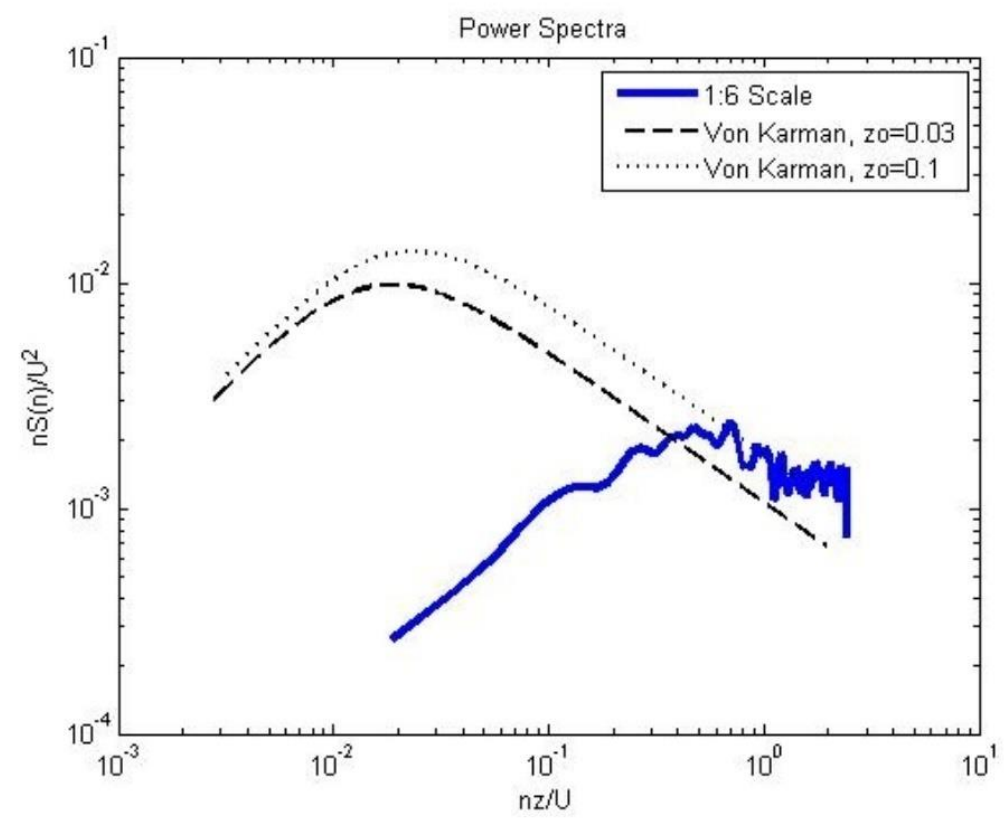

Figure 2 - Comparison of Turbulence Spectra between different model scales and full-scale for a low-rise building of $4 \mathrm{~m}$ height. 


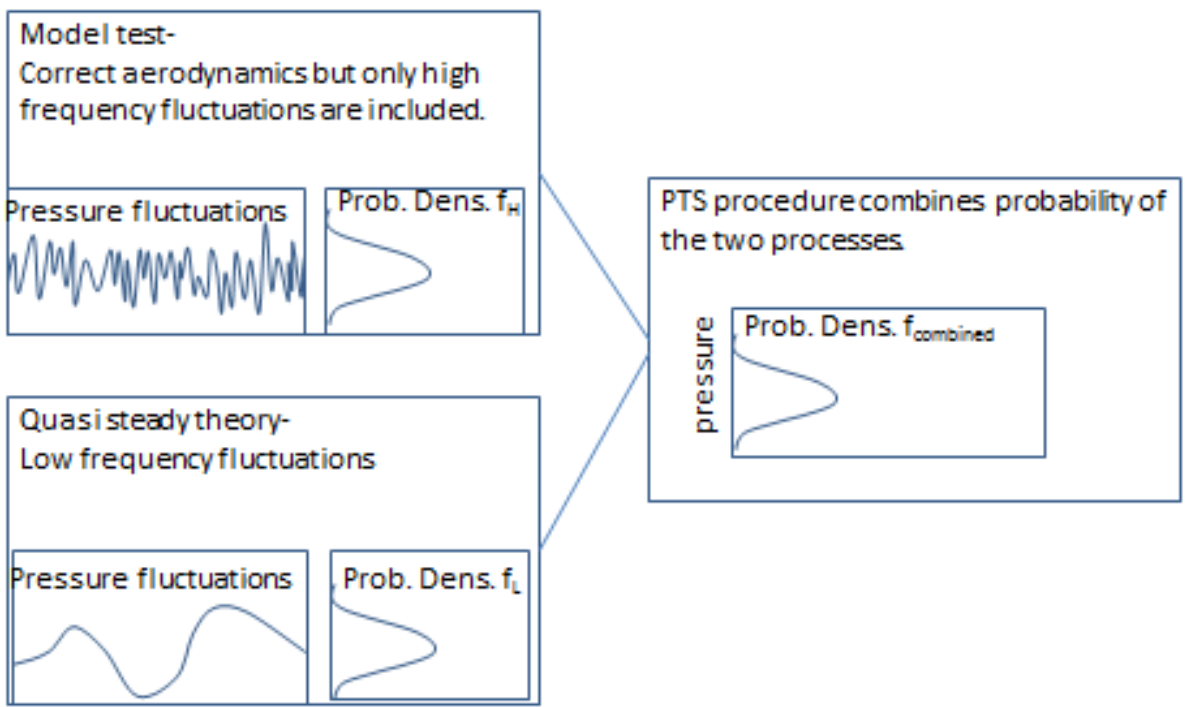

Figure 3 - Flowchart describing the PTS correction method [51]

\subsection{Research Objectives}

The application and evaluation of the partial turbulence simulation method by Asghari et al. [49] was limited to the tests on the TTU building and the Silsoe Cube, and the model scales were 1:6 and 1:5, respectively. The results were very encouraging, but it would be highly desirable to undertake a systematic series of tests at scales covering the complete range from about 1:5 through to 1:100 using the PTS method. One would expect the PTS "corrections" to become progressively smaller as the model scale goes from 1:5 to 1:100 and knowing at what point the correction becomes negligible would be very useful for the wind engineering field in general. Such effect of scaling using large-scale models will be investigated as the first objective of this work. This will be extended to full-scale testing of small structures. 
Also, it is required to explore some simplifications of the PTS method aimed at making it easier to apply in practice. For example, a simplified process will be developed to address the effects of lateral wind speed component fluctuations in addition to missing lowfrequency longitudinal wind speed fluctuations. Thus, the second objective is to simplify the current 3DPTS method by using a weighted average approach. The third objective is to study blockage effects for large-scale models and investigate effects of Reynolds number on mean and peak pressures.

Finally, the last objective is to investigate the application of PTS in the testing of tall buildings. The model scales used for testing tall buildings are typically of the order of 1:200 to 1:600. At these small scales the overall behavior of the structure is well studied, but if there is a need to evaluate the wind effect on building components, then a larger scale testing is required. These large-scale studies can be affected by the missing low-frequency turbulence data. Therefore it will be worthwhile to explore the improvement of the results by incorporating PTS method in the data analysis procedures.

\subsection{Thesis Structure}

An introduction to the effects of scaling on the peak pressure coefficients is provided in the first chapter where the latest efforts in that area is reviewed and reported and the knowledge gap and the unanswered questions are identified. The research objectives and the proposed hypothesis to answer those questions are discussed subsequently. Chapter 2 introduces the Partial Turbulence Simulation (PTS) method which has been developed recently to address the issue of missing low-frequency turbulence content in large-scale wind tunnel experiments. The challenges facing the application of the method are discussed 
followed by some proposed advancements in the method and the application procedure, i.e., the introduction of the weighted average PTS method and determining the range of scales for which the PTS application is required. Chapter 3 describes the methodology and the test plan and also presents the details of the boundary layer simulation and the assessed flow conditions. An extensive presentation and discussion of the results is presented in Chapter 4 where through various plots and illustrations, a comprehensive picture of the outcomes is painted. The reported results are compared to the available field data from the TTU full-scale building and also few other similar studies. The summary and the conclusion are provided in Chapter 5, followed by some recommendations for the future work in Chapter 6. Cited references are listed afterward. The document ends with a series of appendices in which the details of the PTS calculation procedures, data curation and analysis procedures and the developed MATLAB codes and packages are provided, followed by a short discussion of the applicability of the method to estimate to peak wind pressures on tall buildings. 


\section{CHAPTER II}

\section{THEORY AND METHODOLOGY}

\subsection{Introduction to the Partial Turbulence Simulation (PTS) Method}

Partial Turbulence Simulation (PTS) is based on the quasi-steady theory and assumes that if the high-frequency fluctuations in the upcoming flow are adequately captured in the wind tunnel testing, then the effect of the missing low-frequency turbulence on the peak estimation can be compensated analytically. Based on the quasi-steady theory, the surface pressures generated on a building are directly correlated to the upcoming flow turbulence. So, the changes in the pressures can be related to the changes in wind speed and direction. Thus pressure fluctuations correspond to wind fluctuations.

Considering just the longitudinal component of turbulence, the total velocity $U$ at any time instant can be written as:

$$
\widetilde{U}=U+u_{L}+u_{H}
$$

Where $U$ is the mean velocity, $u_{L}$ is the fluctuating component attributed to the low frequency part of the turbulence spectrum, and $u_{H}$ is the fluctuating part attributed to the high frequency end. In a partial turbulence simulation where we are only simulating the high frequency turbulence, the measured mean wind speed basically consists of the mean wind speed $U$ of the corresponding atmospheric flow plus whatever the low frequency component $u_{L}$ is present at the time. So $I_{u H}$, the measured turbulence intensity of the test, can be written as:

$$
I_{u H}=\frac{\sigma_{u H}}{U+u_{L}}
$$

Eq. 2 
If the rapid equilibrium of the turbulence is assumed, then $I_{u H}$ can be considered as a constant. The theory for the equilibrium of the turbulence states that the small-scale turbulence is expected to rapidly adjust to the large-scale turbulence, which means if a low frequency gust occurs which increases the $u_{L}$, then the $\sigma_{u H}$ adjusts quickly to the new amount of energy being fed into the system. And that's because theoretically near the solid boundary layer of an equilibrium layer, the local turbulence energy generation and dissipation are in an approximate balance.

The full-scale turbulence can be written as:

$$
u_{H}=\frac{u_{H}}{\sigma_{u H}}\left(u+u_{L}\right) I_{u H}
$$

Based on these two equations and ignoring higher order terms in the expansions, the fundamental equation for the PTS method is derived as:

$$
I_{u L}=\sqrt{I_{u}^{2}-I_{u H}^{2}}
$$

The detailed derivation is provided in [49].

The next step is to determine the frequency at which the division between low and highfrequency segments of the spectra occurs. Although such a sharp division doesn't exist physically, an approximate point can be calculated. Since the area under the turbulence spectra is equal to the total turbulence intensity, so integrating the spectra from $n_{c}$ to the high frequency end should return the high frequency content of the turbulence:

$$
\left(\frac{I_{u H}}{I_{u}}\right)^{2}=\int_{n_{c}}^{\infty} \frac{S_{u}(n)}{\sigma_{u}^{2}} d n
$$

And if a Von Karman model is used for the spectra, then with some simplifications the Eq. 6 can be derived as an approximate formula to estimate the dividing frequency: 
$n_{c}=0.0716 \frac{U}{{ }^{x} L_{u}}\left(\frac{I_{u}}{I_{u H}}\right)^{3}$

Eq. 6

where $U$ and ${ }^{x} L_{u}$ are the full spectrum values of mean wind velocity and integral length scale, respectively.

In PTS the target is to match the non-dimensional turbulence spectra of model and prototype at high frequencies as expressed in Eq. 7. As discussed earlier, when mainly the high-frequency turbulence is simulated in the wind tunnel, then the effective mean wind speed would be $U+u_{L}$.

$$
\frac{n_{m} S_{u m}}{U_{m}^{2}}=\frac{n_{p} S_{u p}}{\left(U_{p}+u_{L p}\right)^{2}}
$$

Use of a Von Karman model for the turbulence spectrum and considering the requirement for the similarity of the non-dimensionalized frequency, $\frac{n b}{U}$, between model and prototype, leads to the Eq. 8 as the governing relation to ensure the match at the high end of the spectra:

$$
\frac{I_{u m}}{I_{u p}}=\left(\frac{{ }^{x} L_{u m}}{{ }^{x} L_{u p}}\right)^{1 / 3}\left(\frac{b_{p}}{b_{m}}\right)^{1 / 3}
$$

To estimate peak $C_{p}$ values, the sample period is divided into subintervals of sufficient duration that the peak values occurring in them may be treated as independent. The pressure at each subinterval can be written as:

$$
\hat{p}=\frac{1}{2} \rho Q^{2} \hat{C}
$$

Where $\hat{C}$ is the peak pressure coefficient of a subinterval and Q is the mean wind speed of the test. By ignoring the higher order terms of the lateral wind speed fluctuations, the following relation (Eq. 10) can be defined between the $\hat{C}$ and the pressure coefficient of a 
subinterval based on the mean velocity of the full sample period with full spectrum turbulence present:

$$
\hat{C}_{p}=(1+\zeta)^{2} \hat{C}
$$

$$
\text { where }=\frac{u_{L}}{U} \text {. }
$$

For each subinterval, there will be a combination of $\zeta$ and $\hat{C}$. Adopting a Fisher Tippet type I distribution, the probability that a $\hat{C}$ value is not exceeded in any subinterval can be expressed as:

$$
F(\hat{C})=\exp (-\exp (-a(\hat{C}-b)))
$$

Then combining it with the relation defined in Eq. 10, the probability that $C_{p}$ will be exceeded for a given value of $\zeta$ is:

$$
G\left(\frac{\hat{C}_{p}}{b}, \zeta\right)=1-\exp \left(-\exp \left(-a b\left(\frac{\hat{C}_{p} / b}{(1+\zeta)^{2}}-1\right)\right)\right)
$$

And the probability of $\mathrm{Cp}$ being exceeded for all values of $\zeta$ is:

$$
G\left(\frac{\hat{C}_{p}}{b}\right)=\int_{-\infty}^{+\infty} f_{\zeta}(\zeta)\left[1-\exp \left(-\exp \left(-a b\left(\frac{\hat{C}_{p} / b}{(1+\zeta)^{2}}-1\right)\right)\right)\right] d \zeta
$$

where $f_{\zeta}(\zeta)$ is the probability density function of $\zeta$.

If a Gaussian distribution is assumed for the turbulence, as it's believed to be in a generic boundary layer (free from local aerodynamic effects of upwind structures), then the probability of exceedance can be written as Eq. 14, where $\xi=\zeta / I_{u L}$.

$$
G\left(\frac{\hat{C}_{p}}{b}\right)=\int_{-\infty}^{+\infty} \frac{1}{\sqrt{2 \pi}} e^{-1 / 2 \xi^{2}}\left[1-\exp \left(-\exp \left(-a b\left(\frac{\hat{C}_{p} / b}{\left(1+I_{u L} \xi\right)^{2}}-1\right)\right)\right)\right] d \xi
$$

Eq. 14 
$a$ and $b$ are computed from the Fisher Tippet distribution fit and $I_{u L}$ is the missing low frequency turbulence calculated from Eq. 4. Having these values, then the integral can be computed numerically to find the probability of exceedance of a given $\hat{C}_{p} / b$.

If the equivalent full-scale duration of each subinterval is $t_{s}$ and the full-scale test time is $T$, then the probability that the highest value of $\hat{C}_{p} / b$ is exceeded in a subinterval is $G=t_{s} / T$.

Figure 4 shows a graphical display of the numerical integration results at a specific probability level defined by G. More detail is presented in Appendix A1 through a step by step numerical example.

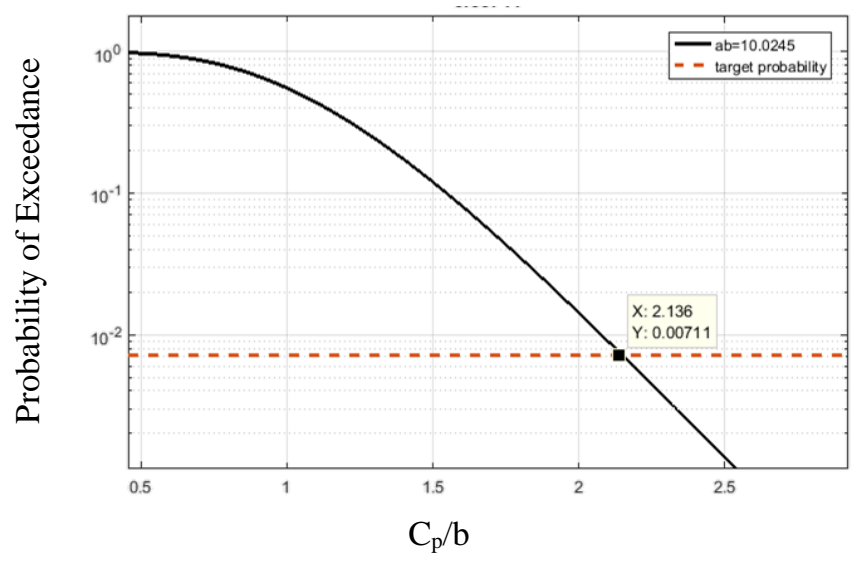

Figure 4 - Numerical solution of the Eq. 14

If other components of the turbulence intensity are considered, i.e., lateral and vertical, then the following triple integral should be solved to obtain the peak $\mathrm{C}_{\mathrm{p}}$ :

$$
G\left(\hat{C}_{p}\right)=\int_{-\infty}^{+\infty} \int_{\frac{\hat{C}_{p}}{1+2 \eta}}^{+\infty} \int_{-\pi}^{+\pi} I_{1}(\hat{C}, \theta) f_{\eta \theta}(\eta, \theta) d \theta d \hat{C} d \eta
$$

$\mathrm{I}_{1}$ is defined by the Eq. 16. 


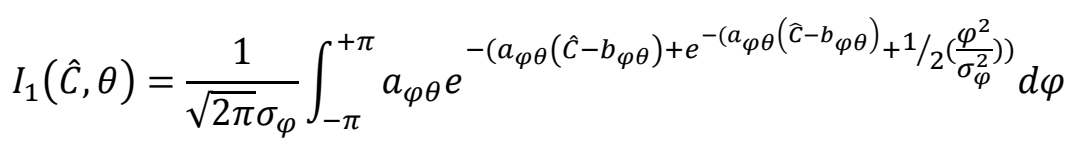

Eq. 16

where $\varphi$ and $\theta$ are the lateral and vertical turbulence intensities respectively, and $\eta=\frac{u_{L}}{U}$.

Since all the three components of the turbulence intensity are included, this method is called the 3DPTS. To do a 3DPTS analysis, a series of data collected at various azimuth (yaw) and pitch angles are required. Figure 5 illustrates a sample range of test directions as are necessary for full three-dimensional analysis. Asghari et al. [49] suggested that data collected at 3-degree increments and up to 15 degrees from either side of the target angle is sufficient enough for an acceptable estimation of peak pressures. Also, they found out the contribution of the vertical turbulence intensity component to the overall accuracy of results was not significant and can be ignored for the sake of simplicity and to reduce the experimental and computational efforts and costs, thus reducing the method to a 2DPTS approach.

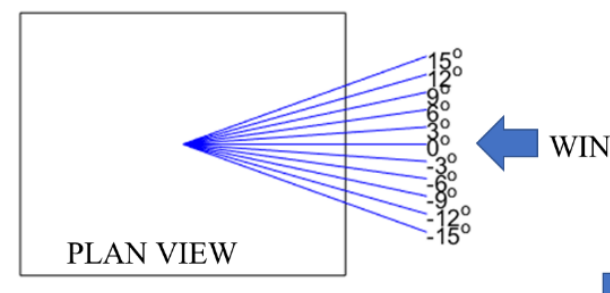

Azimuth (yaw) angle

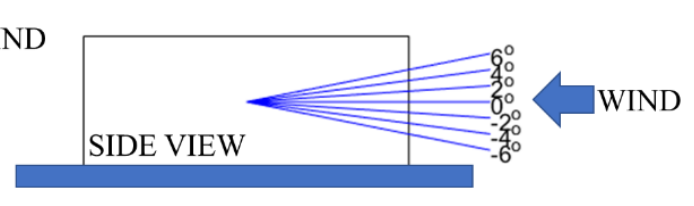

Pitch (tilt) angle

Figure 5 - Range of Azimuth and tilt angles required for a 3DPTS analysis

\subsection{Challenges in the PTS Method}

The PTS method is developed by adopting the basics of the quasi-steady theory, so the limitations of that theory are expected to reflect in the application of the partial turbulence 
simulation method as well. The central assumption of the quasi-steady theory is that the low frequency pressure fluctuations on the surface of a building correspond to the wind speed of the upcoming flow and are in high correlation with the turbulence intensity of the incident flow. But if the low frequency flow is considerably affected by the building, then the quasi-steady approach may show a weak performance in predicting the induced pressures.

The results of a study by Letchford et al. [52] who investigated the application of the quasi-steady theory to the full-scale measurements of the TTU building indicated that the cross-correlation between the pressures and the turbulence intensity of the flow was weakest around the reattachment zones. But the correlation was significantly high at points adjacent to the separation lines and it performed very well for the area averaged pressures for most areas of the roof. Based on the above discussion one may expect to observe an under-prediction of the pressures at the separation zones which will be examined in this study. The other challenge is to have the proper model size to meet the requirements for the validity of the quasi-steady assumption which will be discussed in the subsequent sections of this chapter.

Other challenges for the PTS method are the cost of experiments and the complexity of the analytical process. As mentioned in the previous section, Asghari et al. [49] concluded that a 2D-PTS application would be accurate enough, so there is no requirement to do the tests at different tilt angles since the effect of the vertical component of the turbulence is ignored in the 2D approach. Although the 2DPTS is considerably simpler and faster than the full three-dimensional analysis, for a routine analysis procedure in a wind tunnel still a more convenient and straightforward analytical tool is desirable. The weighted average 
PTS has been developed as part of the present work to address this need and will be discussed in the next session.

\subsection{Proposed Advancements in PTS method}

\subsubsection{Development of the Weighted Average}

The 2DPTS method includes the effect of both longitudinal and lateral turbulence due to the variation of yaw angle (azimuth) in the upcoming wind while the simplified PTS just takes longitudinal turbulence parameters into peak $C_{p}$ calculations. To achieve a 2D level of accuracy by using the simplified approach, a weighting average method is proposed which is discussed in this section.

The probability distribution of wind turbulence in a free stream boundary layer is generally Gaussian or near Gaussian ([52], [53]). If a Gaussian distribution is assumed for the lateral component of wind speed which is denoted as $v$ (Figure 7), then that distribution can be related to the yaw angle through Eq. 17:

$$
\varphi_{\text {rad }}=\frac{v}{\bar{u}}
$$

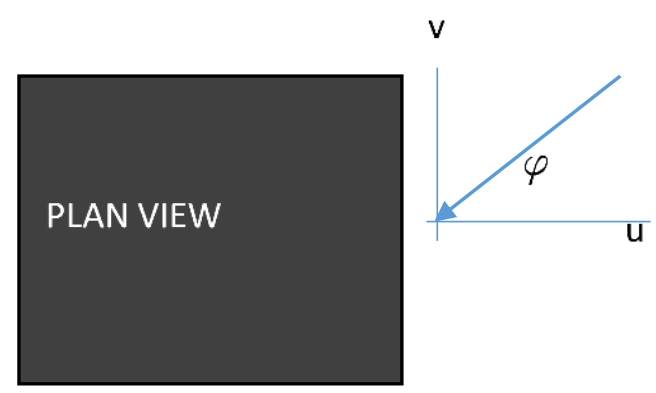

Figure 6 - Definition of the yaw angle 


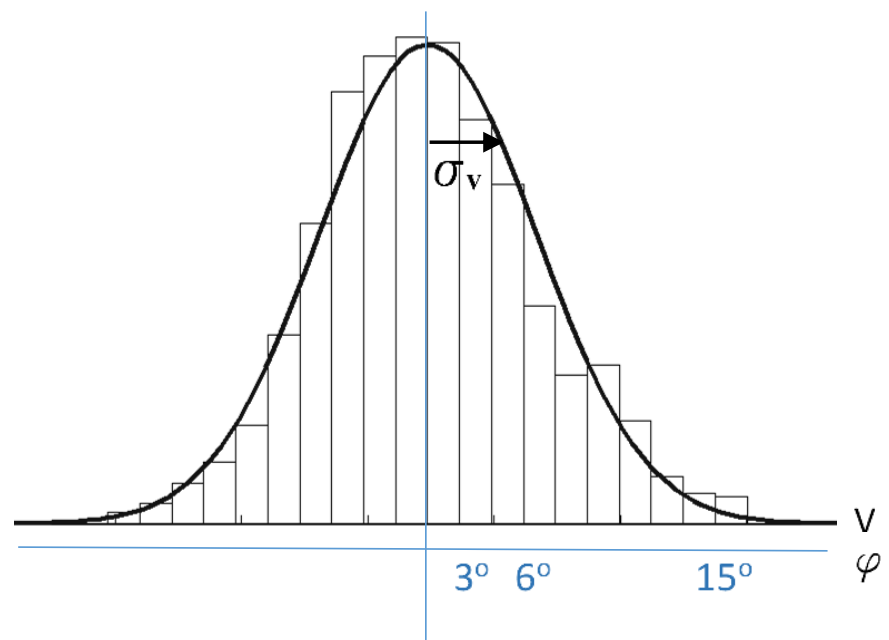

Central Angle

Figure 7 - Distribution of the lateral component of the turbulence

The probability density function for $\phi$ can be written as Eq. 18 :

$$
f(\phi)=\frac{1}{\sqrt{2 \pi} \sigma_{\phi}} e^{-\frac{1}{2}\left(\frac{\phi}{\sigma_{\phi}}\right)^{2}}
$$

Eq. 18

The probability of $\phi$ being in the range of $\phi \pm \frac{1}{2} \delta \phi$ is calculated by Eq. 19:

$$
F\left(\phi \pm \frac{1}{2} \delta \phi\right)=\int_{\phi-\frac{1}{2} \delta \phi}^{\phi+\frac{1}{2} \delta \phi} f(\phi) d \phi \approx f(\phi) \delta \phi
$$

The average value $C_{p 0}$ of $C_{p}(\phi)$ is estimated by Eq. 20:

$$
\begin{aligned}
C_{p 0} & =\int_{-\pi}^{+\pi} C_{p}(\phi) f(\phi) d \phi \approx \sum_{i} C_{p}\left(\phi_{i}\right) f\left(\phi_{i}\right) \delta \phi \\
& =\sum_{i} C_{p}\left(\phi_{i}\right) \frac{1}{\sqrt{2 \pi} \sigma_{\phi}} e^{-\frac{1}{2}\left(\frac{\phi_{i}}{\sigma_{\phi}}\right)^{2}} \delta \phi
\end{aligned}
$$

Eq. 20

The missing component of the lateral turbulence intensity $\left(I_{v L}\right)$ is defined by Eq. 21 : 
$I_{v L}=\sqrt{I_{v}-I_{v H}}$

Eq. 21

where:

$I_{v}:$ Full lateral turbulence intensity obtained from ESDU [54]

$I_{v H}$ : Lateral turbulence intensity obtained from WOW data

Since in the PTS method $\sigma_{\phi}=I_{v L}$ (considering Eq. 18 and Eq. 21) we find that an estimate of the weight average $C_{p}$ is given by Eq. 22:

$$
C_{p 0}=\sum_{i} C_{p}\left(\phi_{i}\right) \frac{1}{\sqrt{2 \pi} I_{v L}} e^{-\frac{1}{2}\left(\frac{\phi_{i}}{I_{v L}}\right)^{2}} \delta \phi
$$

Since we have measurements at 3 -degree intervals, $\delta \phi=\frac{\pi}{60}$ radians.

According to the distribution (Figure 7), moving toward higher values of $\mathrm{v}$ which correspond to higher values of $\phi$, the probability of occurrence declines which indicates that the contribution of larger angles should be expected to be less as departing from the central angle. So, the probability distribution function can be used as a weighting function to calculate the peak $C_{p}$ at the central angle by averaging over a range of lateral angles, hence including the effect of lateral turbulence.

\section{Derivation}

$$
\varphi_{\text {rad }}=\frac{v}{\bar{u}} \rightarrow \sigma_{\varphi}=\frac{\sigma_{v}}{\bar{u}}=I_{v}
$$




\subsubsection{Investigation of Scaling Effects}

The applicability of the quasi-steady theory is uncertain for the pressures in the highfrequency range of the pressure power spectrum [55]. Letchford et al. [52] examined this limitation by comparing the predicted results of a quasi-steady approach versus the fullscale TTU data and concluded that at the smaller values of the eddy wavelength $\lambda=U / n$, the discrepancy increases, and the theory becomes incapable of an accurate prediction. Therefore, to remain in the range of quasi-steady validity, higher wavelengths should be sought relative to the model dimensions. This can be expressed in terms of a $\lambda / \mathrm{b}$ ratio where $\mathrm{b}$ is the height of the building model. Considering Eq. 6 and Eq. 8, at the dividing frequency, $\mathrm{n}_{\mathrm{c}}$, this $\lambda / \mathrm{b}$ ratio approximately is equal to $14 \times \mathrm{L}_{u m} / \mathrm{b}_{\mathrm{m}}$. So, if for instance, the $\lambda / \mathrm{b}$ ratio were to be set to $\lambda / b>10$, then $L_{u m} / b_{m}$ should be higher than 0.7 . The largest model in the current study that is a 1:6 model of the TTU building, has a $\mathrm{L}_{u m} / \mathrm{b}_{\mathrm{m}}$ ratio of 0.78 which can be on the borderline of applicability of the PTS method. It is essential to know the range of applicability of the PTS method and also to determine the conditions in which a PTS correction should be incorporated in the peak estimation process. To achieve these, a range of model scales was selected to build the TTU building scaled models, and the results were compared to the corresponding field data. At the very small scales where the full turbulence content is obtainable, the effect of the missing low-frequency turbulence on the accuracy of results is minimal, and so is the degree of the PTS correction consequently. However, due to a considerable violation of the Reynolds number similarity between a small model and the prototype, there may be a substantial difference between the obtained peaks as well. To produce the required data to investigate both the scaling and the $\operatorname{Re}$ 
effects, the tests were conducted at three wind speed levels to generate various levels of Reynolds number. 


\section{CHAPTER III}

\section{DESIGN OF EXPERIMENTS AND ANALYSIS PROCEDURES}

\subsection{Test Buildings, Instrumentation, and Test Protocol}

The full-scale 12-fan Wall of Wind (WOW) open jet facility (Figure 8a) at Florida International University (FIU) was used to generate the wind field and perform experiments for the reported study [56]. The 12-fan WOW can produce up to and including a SaffirSimpson Category 5 hurricane wind speed that reasonably replicates mean wind speed and turbulence characteristics of real hurricane winds. A set of triangular spires and floor roughness elements, as shown in Figure 8 and Figure 9, were used to generate turbulent atmospheric boundary layer (ABL) characteristics.

The mean wind speed profile and turbulence characteristics of ABL for an open terrain exposure were simulated at the WOW. A series of experiments were conducted at the WOW to measure the vertical wind-speed profile throughout the boundary layer across a range of free-stream wind speeds. Wind speeds were measured at various heights using Turbulent Flow Cobra probes capable of resolving the 3-components of velocity and local static pressure at a sampling rate of $2500 \mathrm{~Hz}$. A schematic of the probes and the convention for wind direction notation, along with the definition of the pitch and yaw angles, are shown in Figure 10. To ensure the devices are well calibrated and as a verification, several simultaneous and even separate measurements were done using pitot tubes as well. 


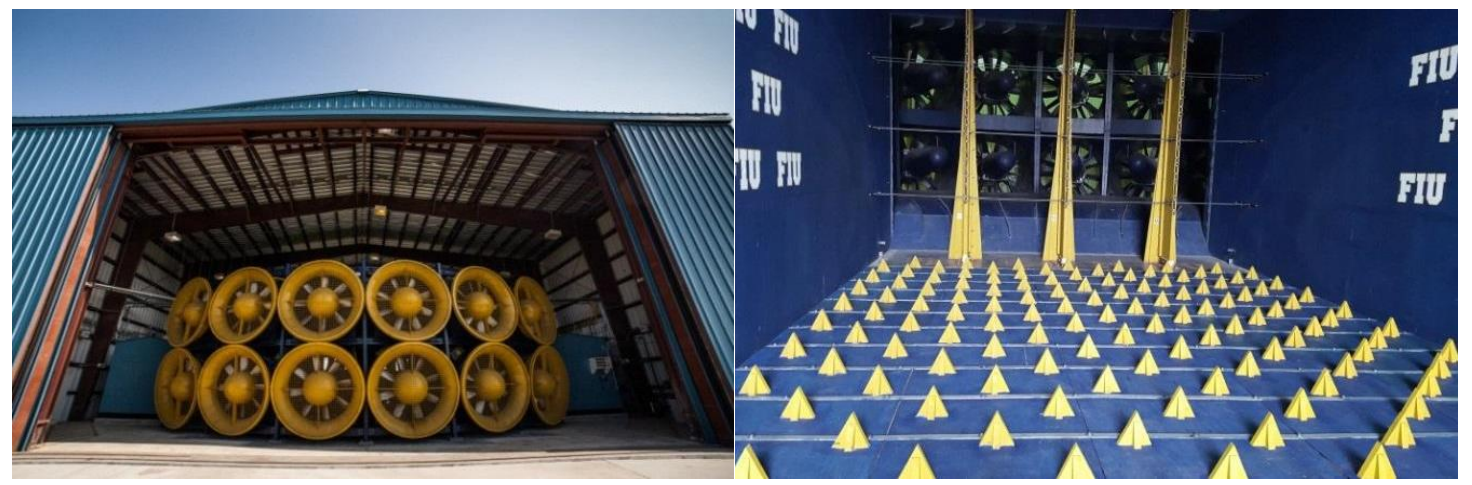

(a)

(b)

Figure 8 - a: Wall of Wind, Florida International University; b: Spires and floor roughness elements

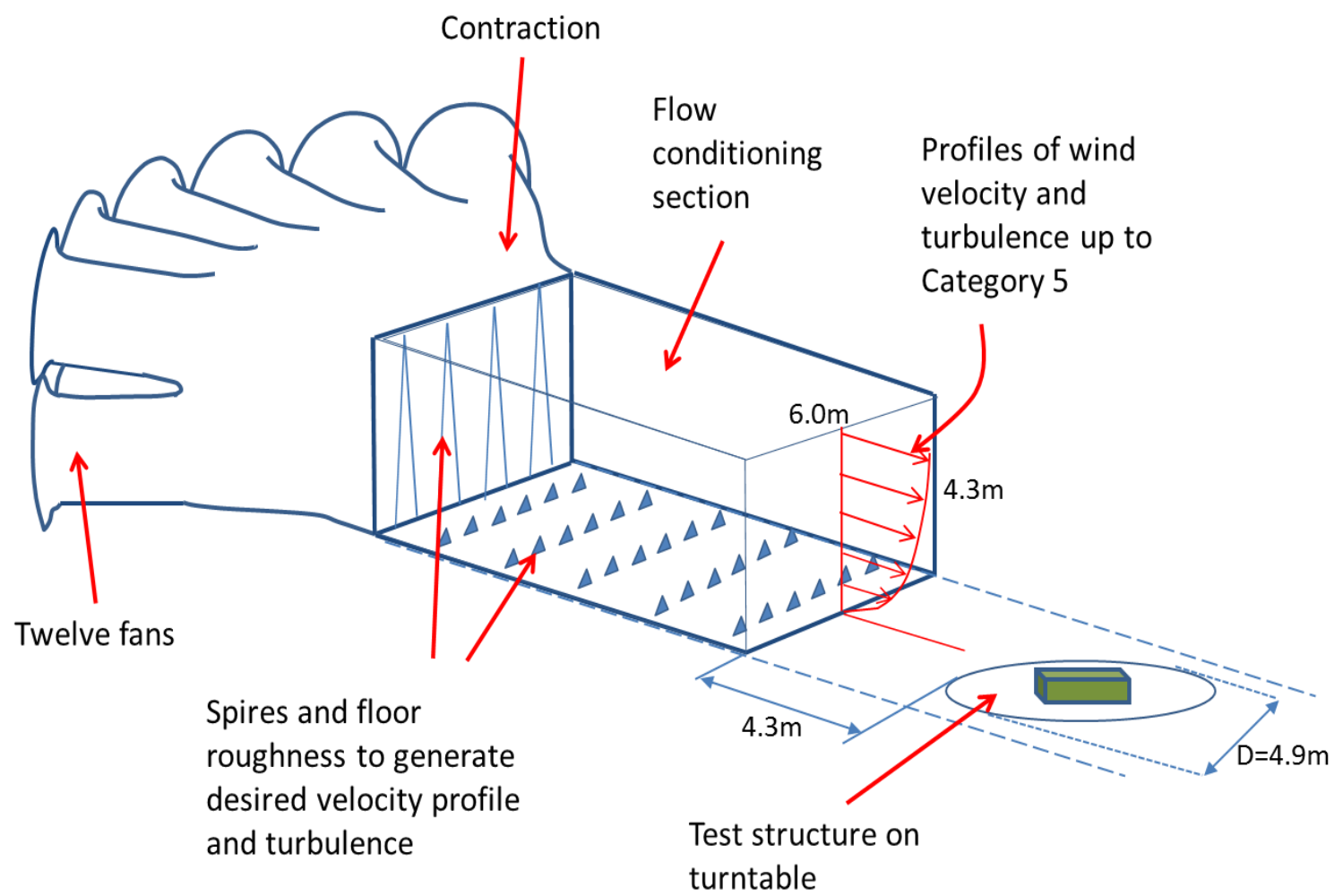

Figure 9 - Schematic drawing of ABL flow generation components for 12-fan WOW 


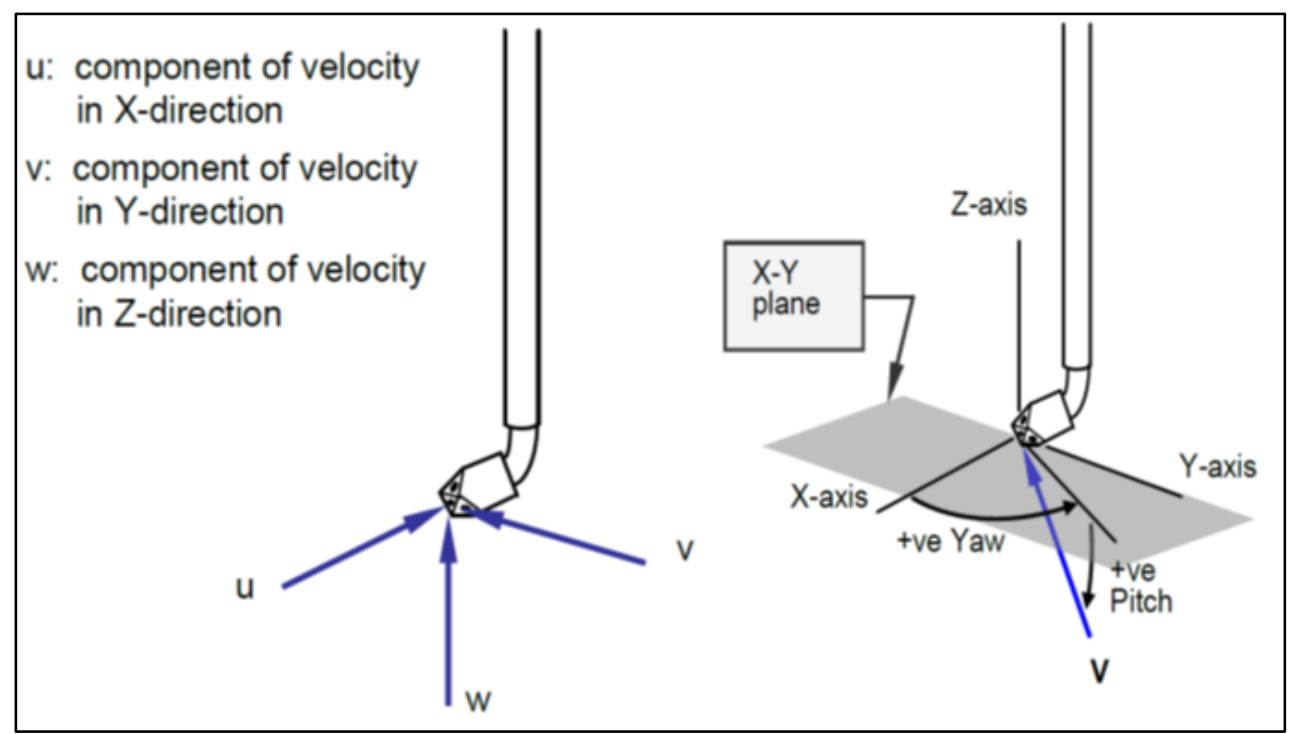

Figure 10 - Conventional notation for the 3 components of wind speed measured by Cobra probes

The surface pressure distribution on the test buildings was measured with $2 \mathrm{~mm}$ diameter pressure taps which were connected to a Scanivalve Pressure Scanning System. This 512 Channel pressure scanning system was used to measure the pressure time histories on the model buildings walls and roofs. The pressure transducers were connected to a set of temperature control units (TCU), and TCUs were connected to a Digital Service Module DSM 4000 that transfers the information to the data acquisition system, where pressure data were collected at a sampling rate of $512 \mathrm{~Hz}$. The static port of the device was tubed to a pressure bottle outside the building to measure the static pressure.

Based on the model dimensions and observing the accessibility and workability, long enough tubes were used to connect the pressure taps to the pressure scanners (i.e., ZOCs). Afterward, the collected data from these tubes were corrected for the tubing distortion effects using proper transfer functions [57]. More detailed information on the transfer function application is provided in Appendix 0. 
The collected wind pressures were normalized with reference to the dynamic wind pressure at the building eave height to obtain pressure coefficients using Eq. 23 and Eq. 24:

$$
\begin{gathered}
C_{p \text { mean }}=\frac{P_{\text {mean }}}{\frac{1}{2} \rho U_{\text {mean }}^{2}} \\
C_{\text {p peak }}=\frac{P_{\text {peak }}}{\frac{1}{2} \rho U_{\text {mean }}^{2}}
\end{gathered}
$$

where $P_{\text {mean }}$ and $P_{\text {peak }}$ are mean and peak differential pressure for a specific tap, $\rho$ is the air density and $\mathrm{U}_{\text {mean }}$ is the mean and peak wind speed at the building eave height, respectively.

\subsubsection{Test Models}

The following table shows the dimensions of the prototype building and the five model scales considered in the current study. Figure 11 shows a schematic of the building. A picture of the TTU and the surrounding terrain is shown in Figure 12 while a closer view of the surface condition and the pressure taps is provided in Figure 13.

Table 1 - Prototype and model dimensions

\begin{tabular}{|r|r|r|r|}
\hline Scale & B $(\mathrm{m})$ & $\mathrm{L}(\mathrm{m})$ & $\mathrm{H}(\mathrm{m})$ \\
\hline Full $(1: 1)$ & 9.14 & 13.72 & 3.96 \\
\hline $1: 6$ & 1.52 & 2.29 & 0.66 \\
\hline $1: 10$ & 0.91 & 1.37 & 0.40 \\
\hline $1: 20$ & 0.46 & 0.69 & 0.20 \\
\hline $1: 50$ & 0.18 & 0.27 & 0.08 \\
\hline $1: 100$ & 0.09 & 0.14 & 0.04 \\
\hline
\end{tabular}

Building walls and roof were equipped with 204 pressure taps in total. Since the comparison of the results with their full-scale counterparts is the core part of the study, tap locations were selected based on the full-scale tap locations (Figure 14) in an effort to fully 
replicate the details of the TTU test building. Figure 15 illustrates the pressure tap locations and numbering. Detailed drawings of the models and the location of taps are included in Appendix 0.

To build the 1:100 and 1:50 models initially FDM (Fused Deposition Modeling) 3D printing technology was considered, and sample models were built. But since the model dimensions were very small and the correct modeling of the details and tap locations, especially the corner taps, were of utmost importance, they were ordered to be built by RWDI using stereolithography 3D printing technology. The other models were constructed using Plexiglas sheets over wood framing. Figure 16 to Figure 20 show the built models.

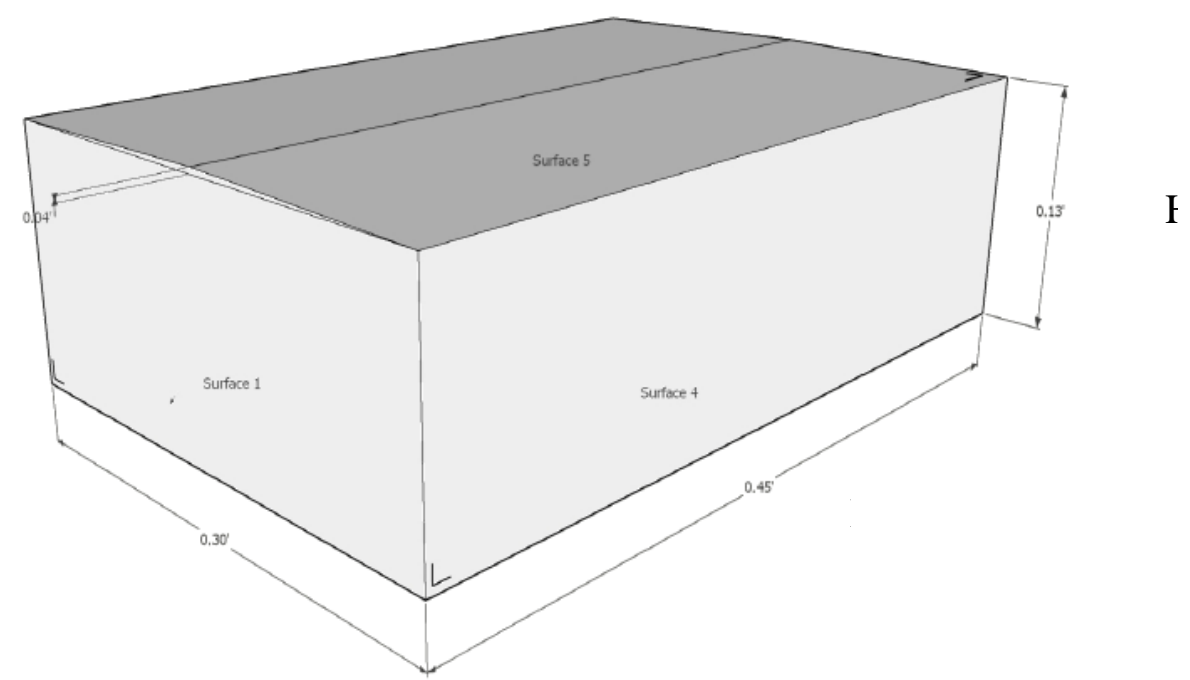

Figure 11 - Schematic of the TTU building model 


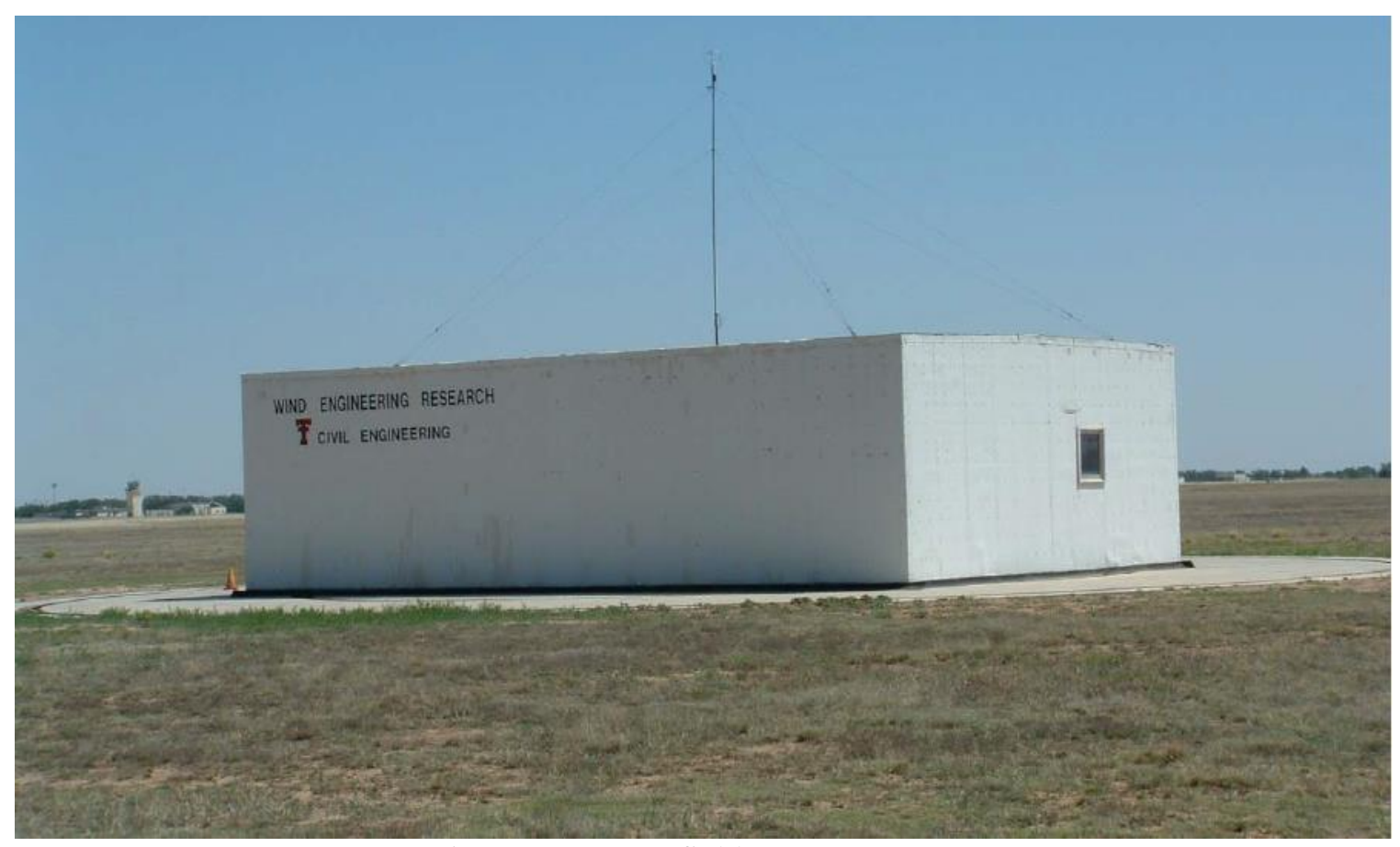

Figure $12-$ TTU field test structure

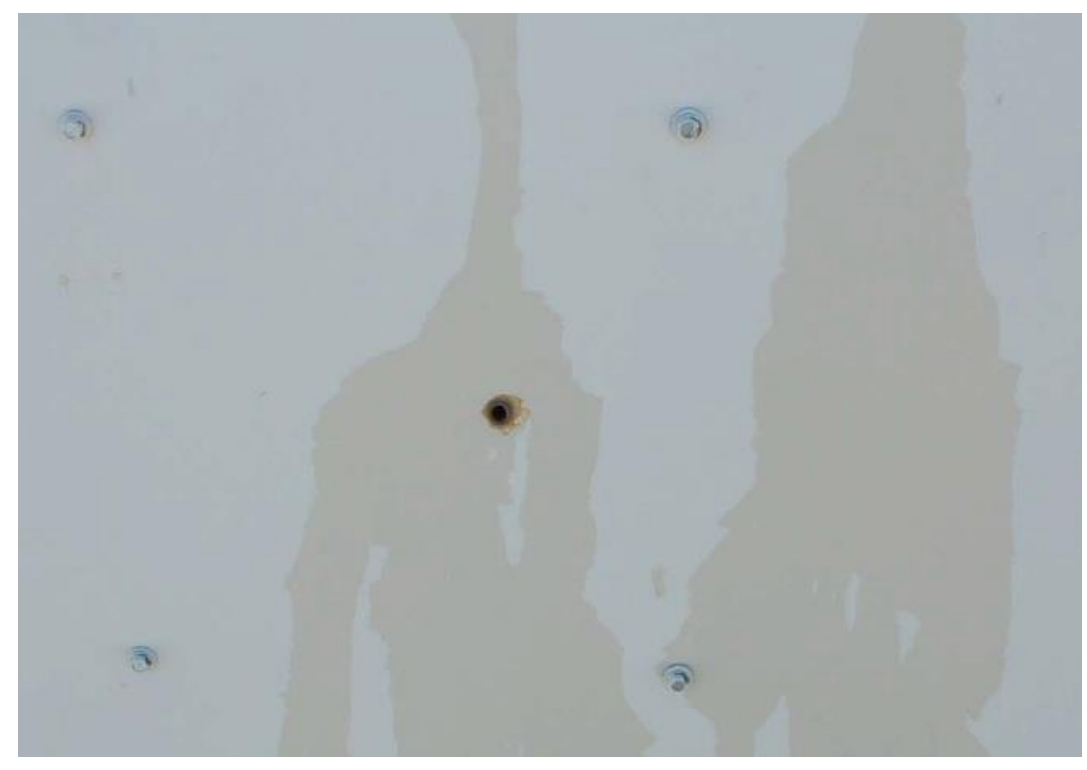

Figure 13 - Pressure tap installation on the TTU full-scale building 


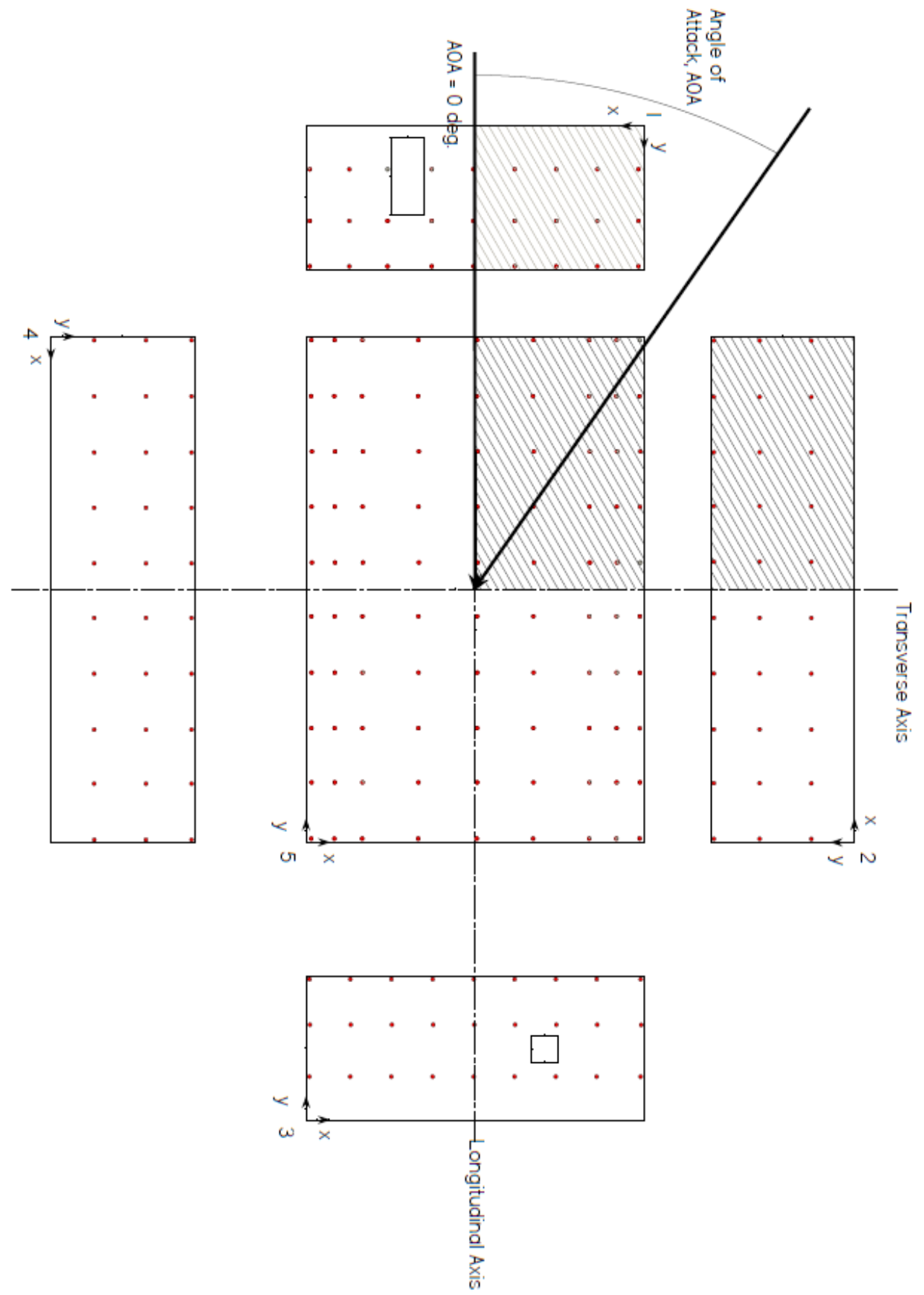

Figure 14 - Pressure tap layout on the TTU full-scale building 
Connected taps, Dir=0,SCALE 1:100

\begin{tabular}{|ccccccccc|}
\hline 27 & 26 & 25 & 24 & 23 & 22 & 21 & 20 & 19 \\
18 & 17 & 16 & 15 & 14 & 13 & 12 & 11 & 10 \\
9 & 8 & 7 & 6 & 5 & 4 & 3 & 2 & 1
\end{tabular}
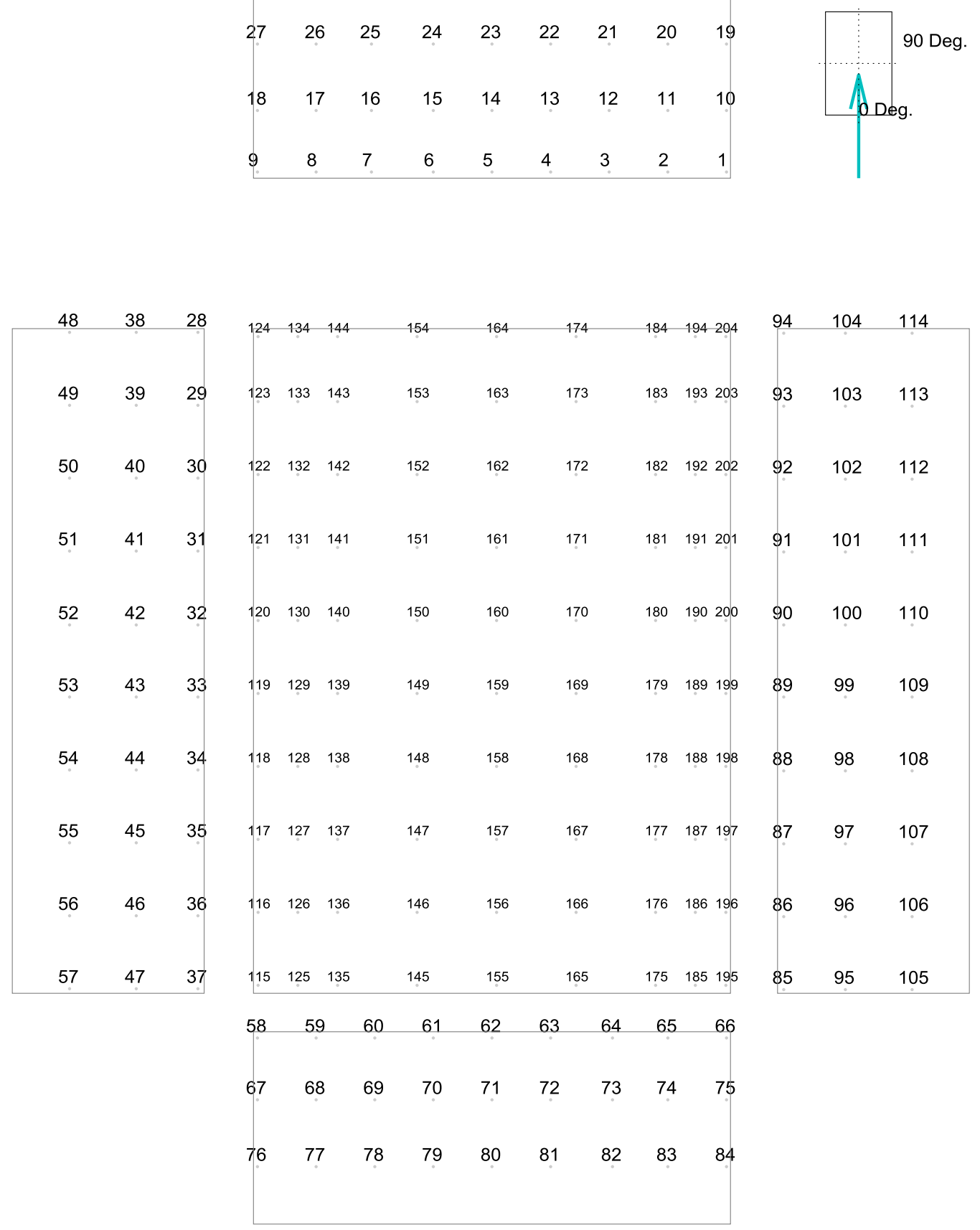

Figure 15 - Tap numbering and location map 

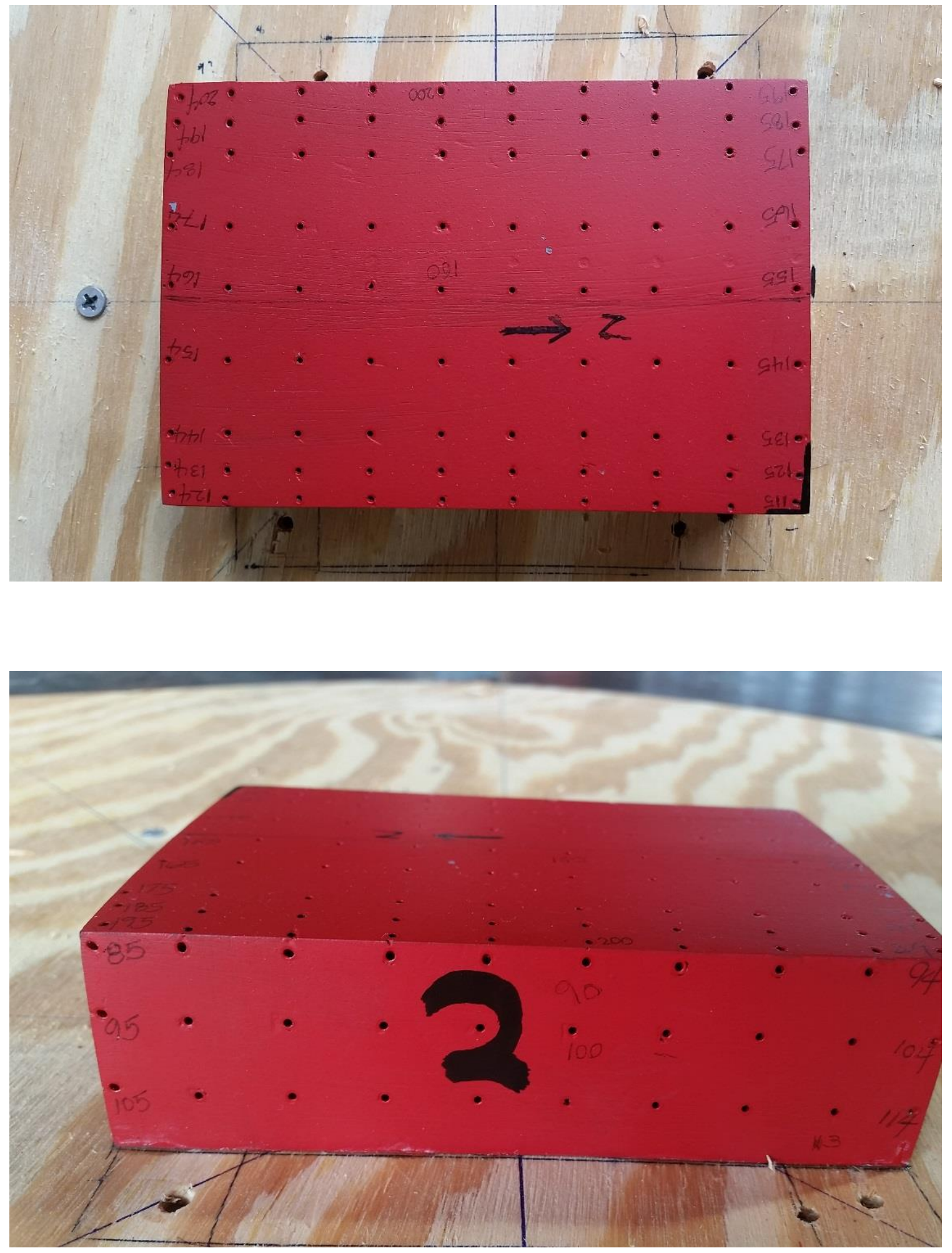

Figure $16-1: 100$ scale model built by the stereolithography technique 

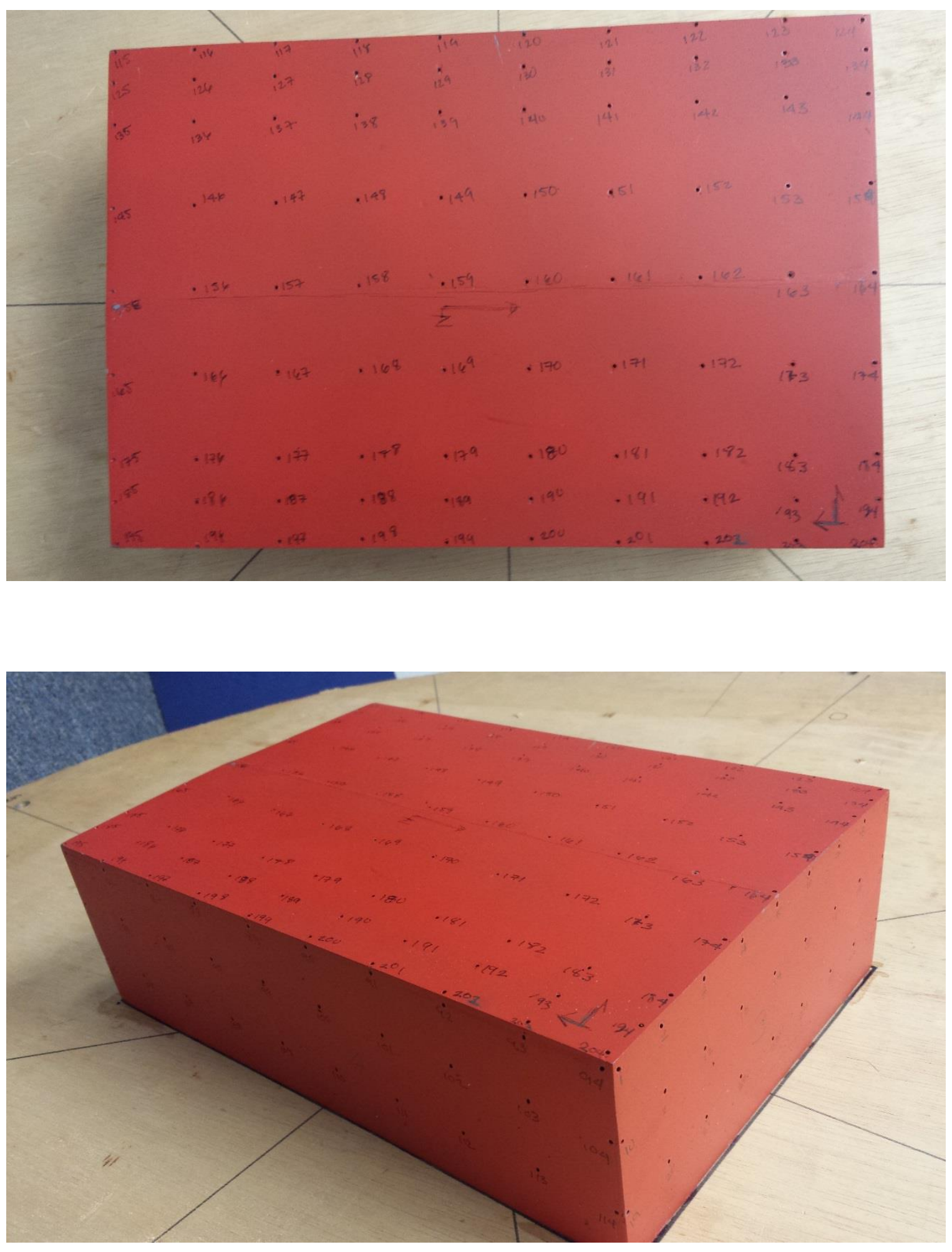

Figure $17-1: 50$ scale model built by the stereolithography technique 


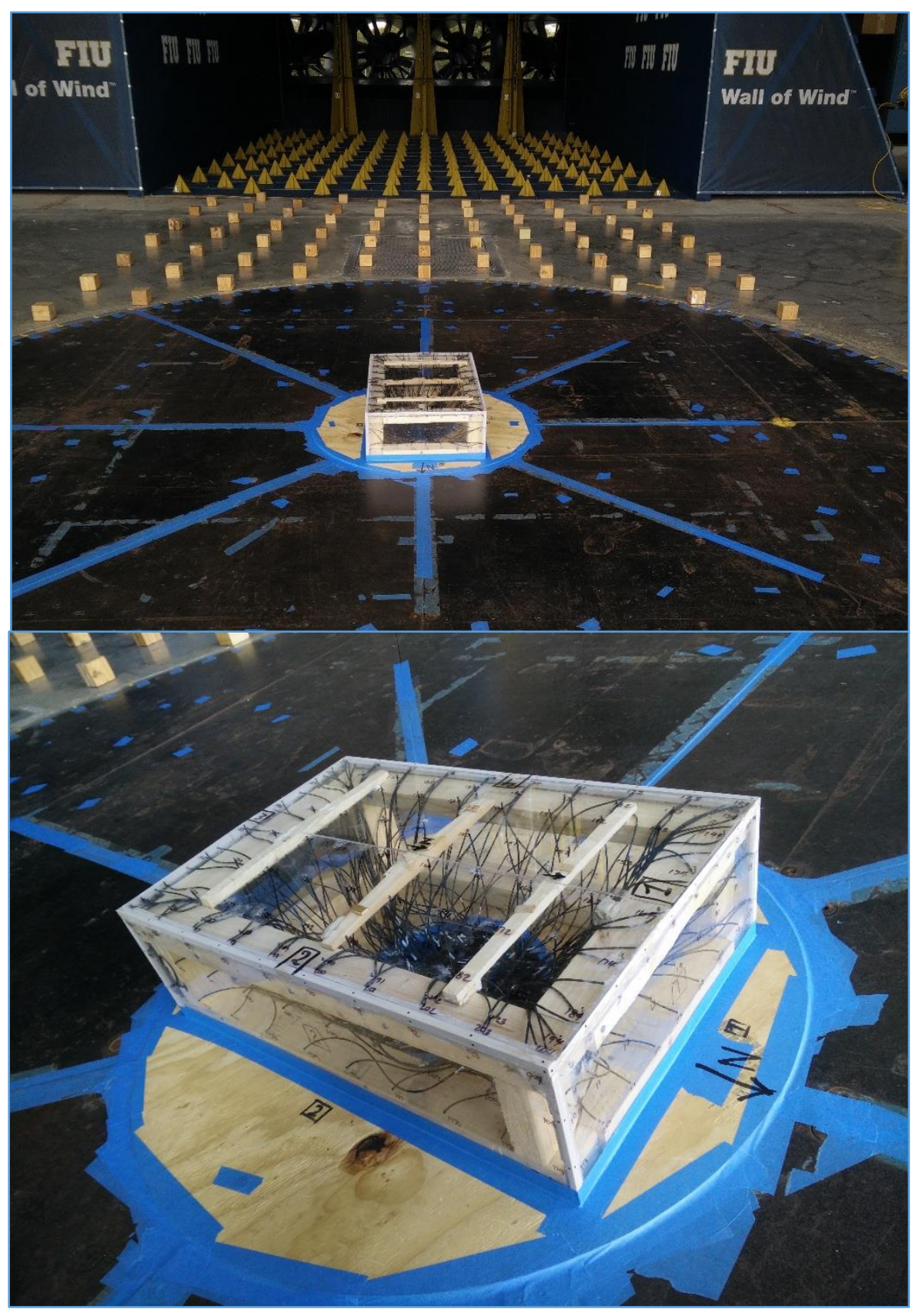

Figure 18 - 1:20 scale model using Plexiglas sheets over wood framing 

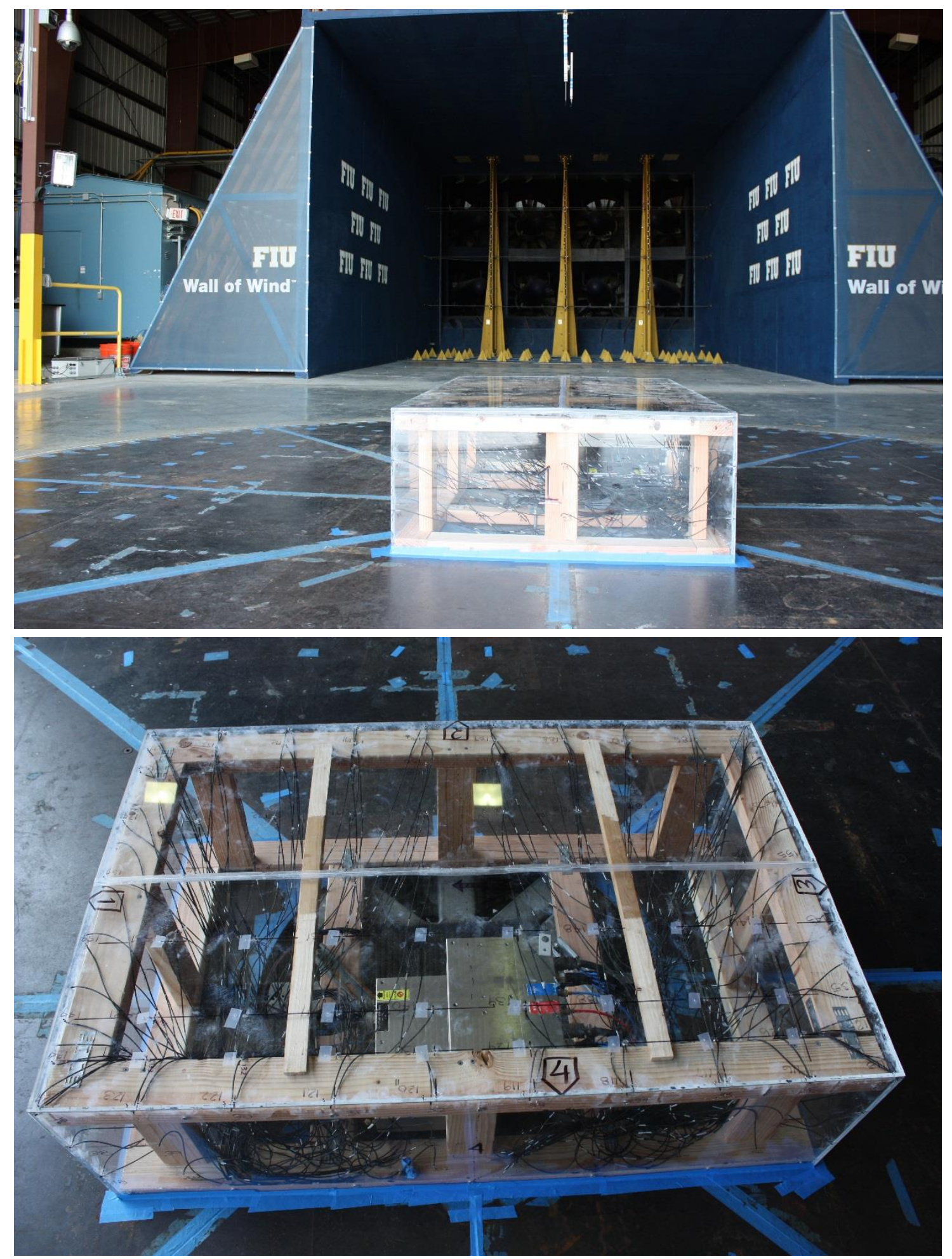

Figure 19-1:10 scale model using Plexiglas sheets over wood framing 


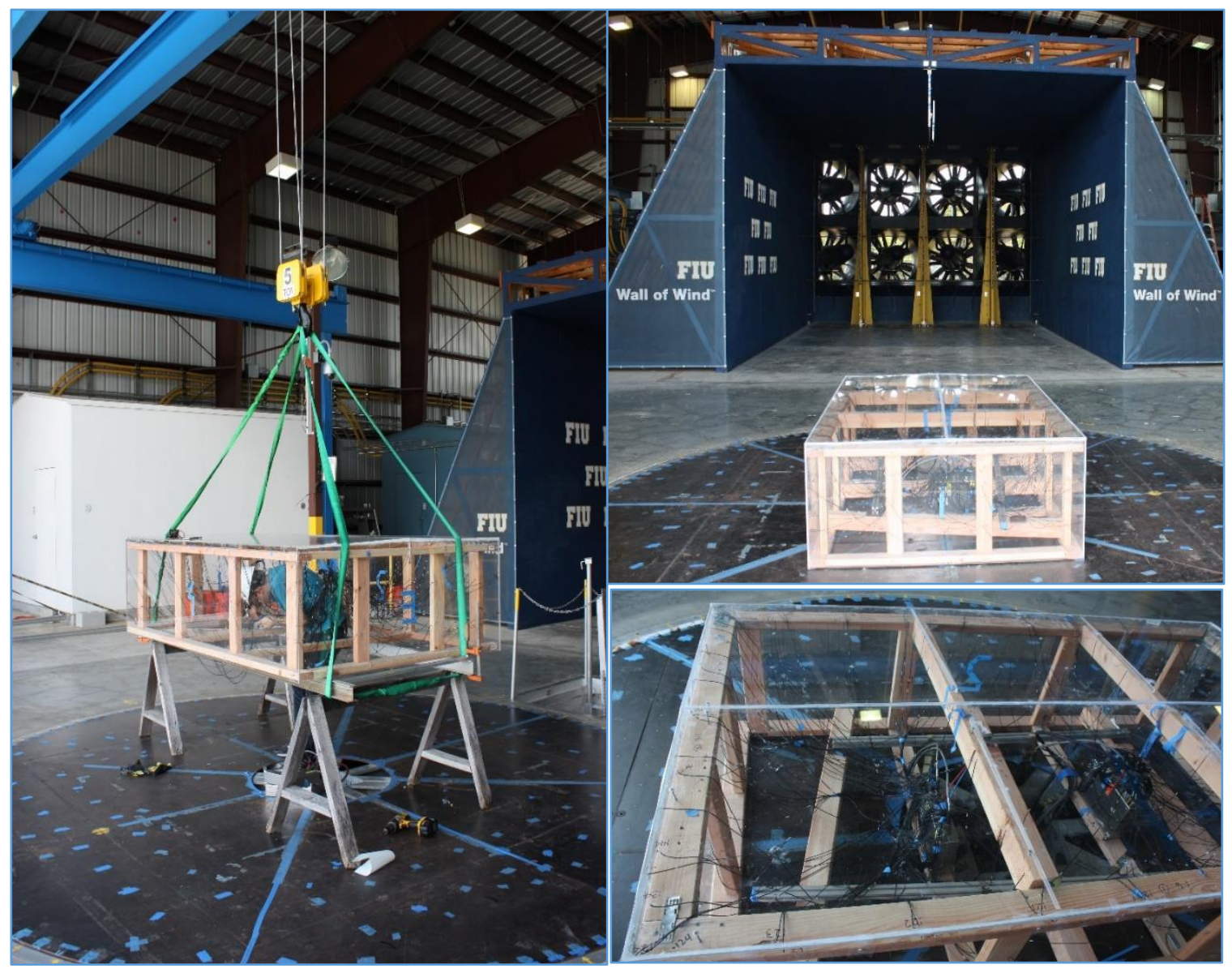

Figure 20 - 1:6 scale model using Plexiglas sheets over wood framing

\subsubsection{Test Protocol}

Building models were tested at wind directions ranging from $0^{\circ}$ to $195^{\circ}$, and also from $345^{\circ}$ to $360^{\circ}$, at 3 -degree increments. Testing was performed for a time duration of 60 seconds for each case. Previous studies [49] have shown that this small increment in the wind direction provides the resolution required to capture enough lateral fluctuations in the oncoming wind. Figure 21 shows the convention for wind directions and the model placement on the turntable. 

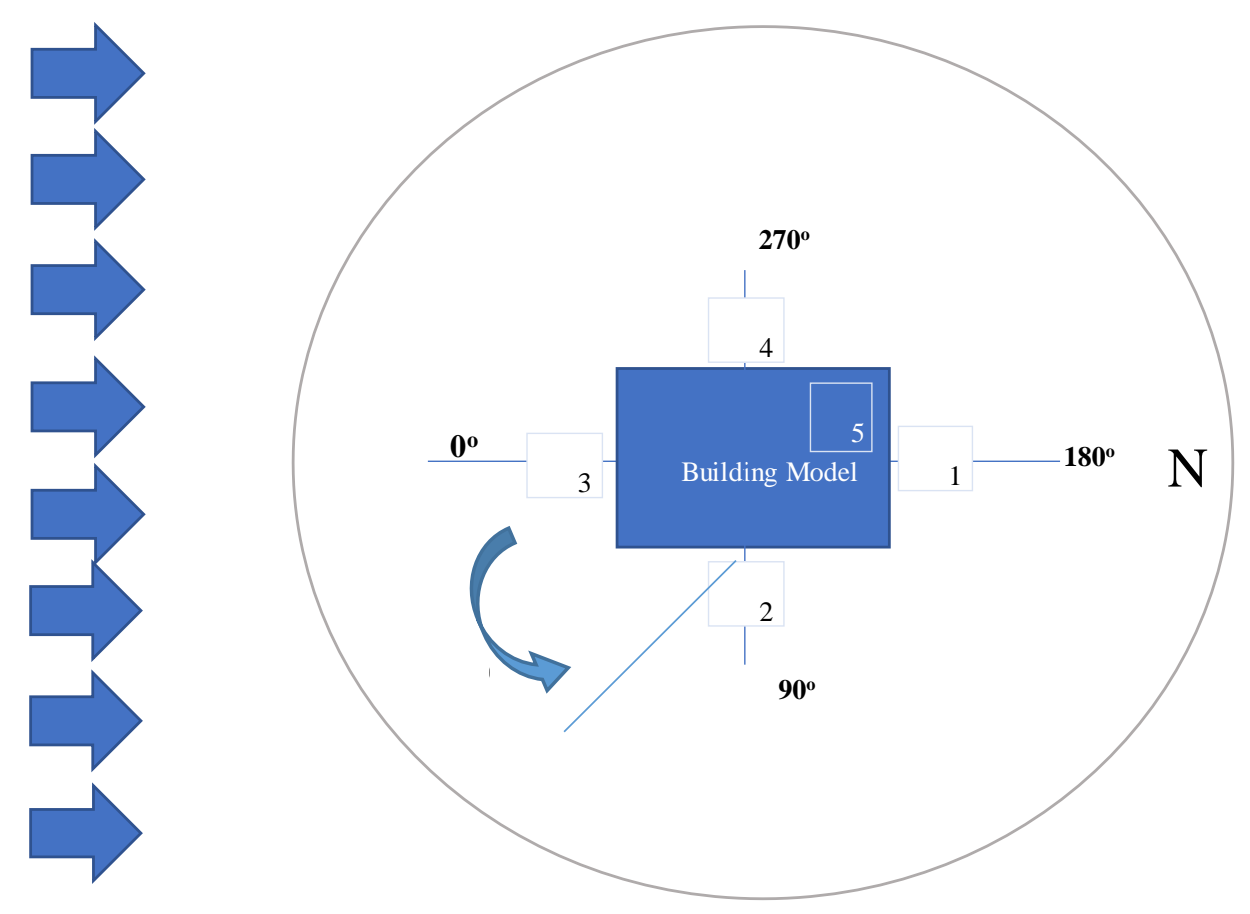

Figure 21 - Convention for the wind direction. Side walls are numbered from 1 to 4 while the roof is labeled as side 5 .

The fan throttle for the conventional testing was set to $40 \%$, but to study the effects of Reynolds number, models were tested at $70 \%$ and $100 \%$ fan throttle for limited directions of $75^{\circ}-105^{\circ}$ and $120^{\circ}-150^{\circ}$. The obtained wind speed at each throttle is reported in the next section.

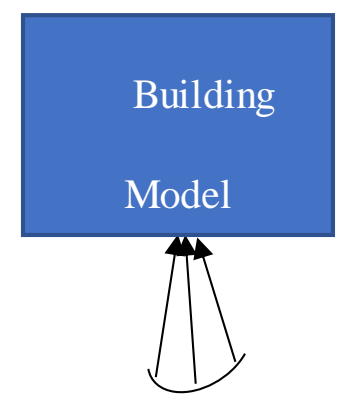

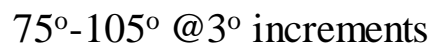

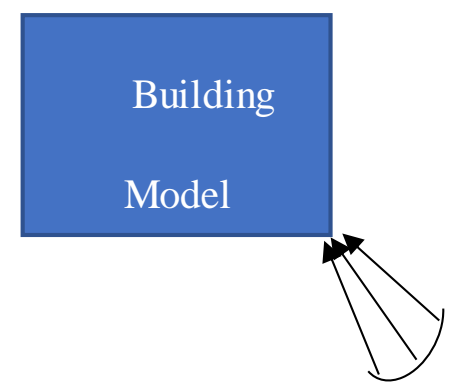

$120^{\circ}-150^{\circ} @ 3^{\circ}$ increments

Figure 22 - Selected wind angles for Reynolds number tests 


\subsection{Boundary Layer Simulation}

Spires and roughness elements are used in FIU Wall of Wind (WOW) to simulate the Atmospheric Boundary Layer (ABL) pertaining to various terrain conditions. The roughness element size should be appropriately scaled with respect to the model scale to maintain the Jenson number, which is the ratio of the model height to the roughness element height, between all the models and to ensure a proper scaled flow condition is generated for each model. Besides that, and to meet the requirements of the PTS, the ratio of the modeled turbulence to the full scale should satisfy the Eq. 8. To achieve these, the customized roughness elements were extended beyond the WOW flow management box as shown in Figure 23 for 1:100 model. The design of the roughness elements was based on an analytical calculation followed by experimental trials. Details are discussed in the next section.

\subsection{Roughness Elements Calculation}

The size and spacing of the extended roughness elements were calculated based on the relation suggested by Irwin [58], which originates from the model developed by Lettau [59] and reflected in the ASCE7-10 commentary (see Eq. 25).

$$
z_{0}=0.5 H_{o b} \frac{S_{o b}}{A_{o b}}
$$

where,

$\mathrm{z}_{0}=$ roughness height

$\mathrm{H}_{\mathrm{ob}}=$ the average height of the roughness in the upwind terrain

$\mathrm{S}_{\mathrm{ob}}=$ the average vertical frontal area per obstruction presented in the wind 
$A_{\mathrm{ob}}=$ the average area of ground occupied by each obstruction, including the open area surrounding it.

Table 2 summarizes the calculations for the roughness elements for a target open terrain condition with $\mathrm{z}_{0}=0.02 \mathrm{~m}$. These estimated values were used as a basis for the experimental study of the terrain and the scaled down boundary layer simulation. Several roughness element layouts were tried at each scale, and the profiles and spectra were compared to those of full-scale to ensure that a proper flow condition is assessed. Figure 23 and Figure 24 show the installation of cylindrical roughness elements for the 1:100 and 1:50 model respectively. The size of the elements was smaller on the inner ring around the model, resulting in a denser layout to deliver the required frontal area. Figure 25 shows the roughness element extension layout for the 1:20 model using the cubic elements. For the 1:10 and 1:6 model, the required roughness and the flow condition was assessed by partially or completely removing the triangular roughness elements as shown in Figure 26 and Figure 27 respectively.

Table 2 - Roughness element size calculations

\begin{tabular}{|c|c|c|c|c|c|c|c|c|c|}
\hline Scale 1:x & $\begin{array}{l}\mathrm{z}_{\mathrm{o}} \text { : Full } \\
\text { Scale }\end{array}$ & $z_{0}:$ Scaled & Spacing (in) & Spacing $(m)$ & $A_{o b}$ (in2) & $A_{o b}(m 2)$ & $\mathrm{H}_{\mathrm{ob}}$ (in) & $H_{o b}(m)$ & \begin{tabular}{|l|} 
Frontal \\
Area \\
(in2) \\
\end{tabular} \\
\hline 10 & 0.02 & 0.00200 & 24 & 0.610 & 576 & 0.372 & 4.493 & 0.114 & 20.188 \\
\hline 20 & 0.02 & 0.00100 & 24 & 0.610 & 576 & 0.372 & 3.566 & 0.091 & 12.718 \\
\hline 50 & 0.02 & 0.00040 & 12 & 0.305 & 144 & 0.093 & 1.655 & 0.042 & 2.740 \\
\hline 100 & 0.02 & 0.00020 & 12 & 0.305 & 144 & 0.093 & 1.314 & 0.033 & 1.726 \\
\hline 100 & 0.02 & 0.00020 & 12 & 0.305 & 144 & 0.093 & 1.314 & 0.033 & 1.726 \\
\hline 180 & 0.02 & 0.00011 & 12 & 0.305 & 144 & 0.093 & 1.080 & 0.027 & 1.166 \\
\hline
\end{tabular}




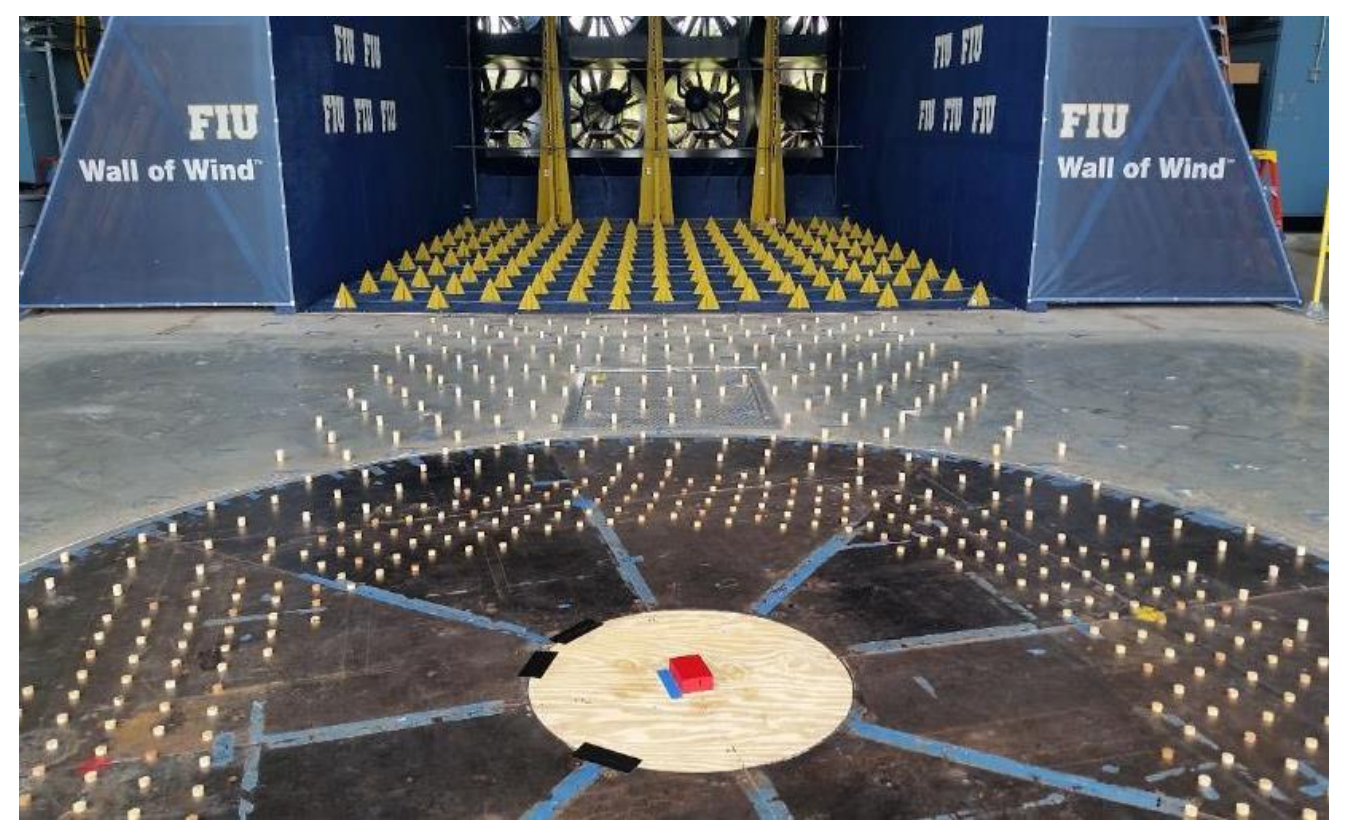

Figure 23 - Extension of roughness elements for 1:100 model

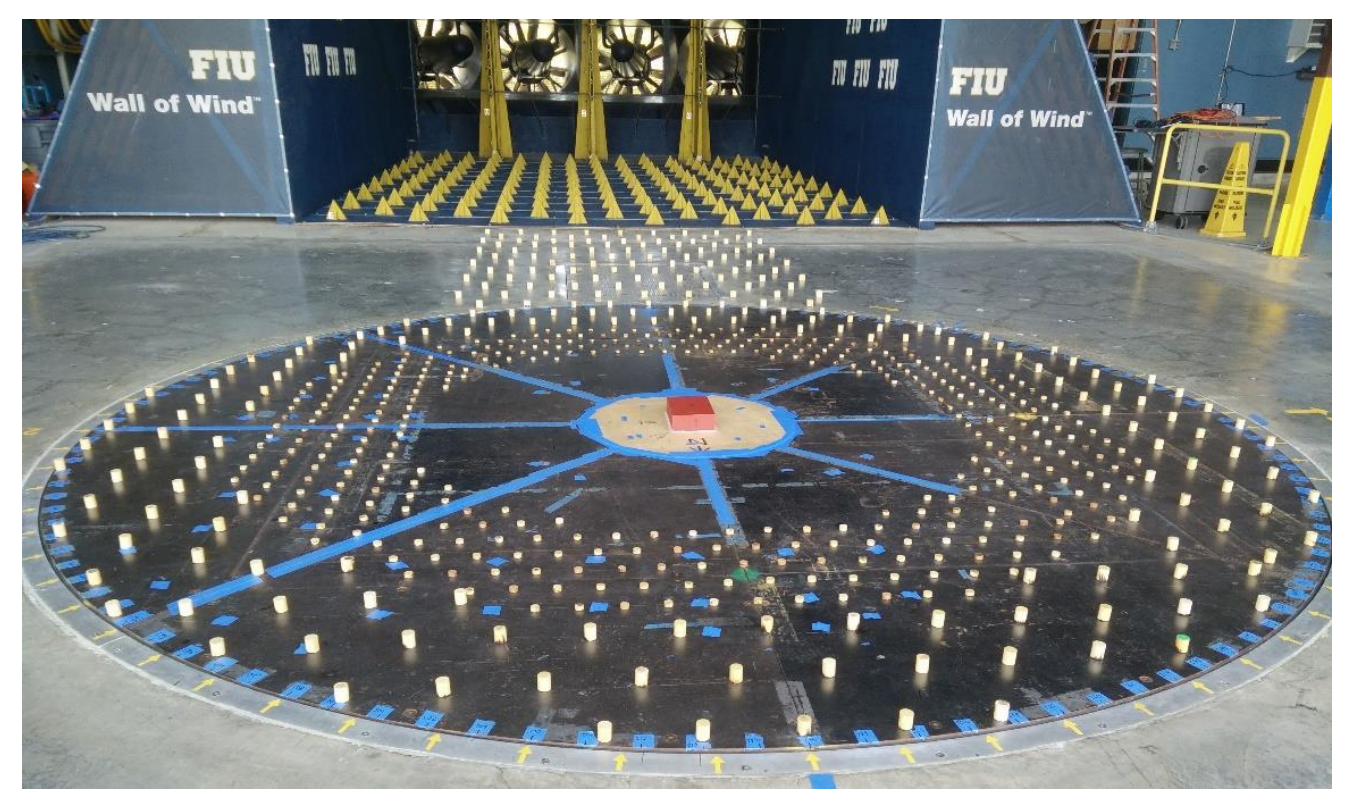

Figure 24 - Extension of roughness elements for 1:50 model 


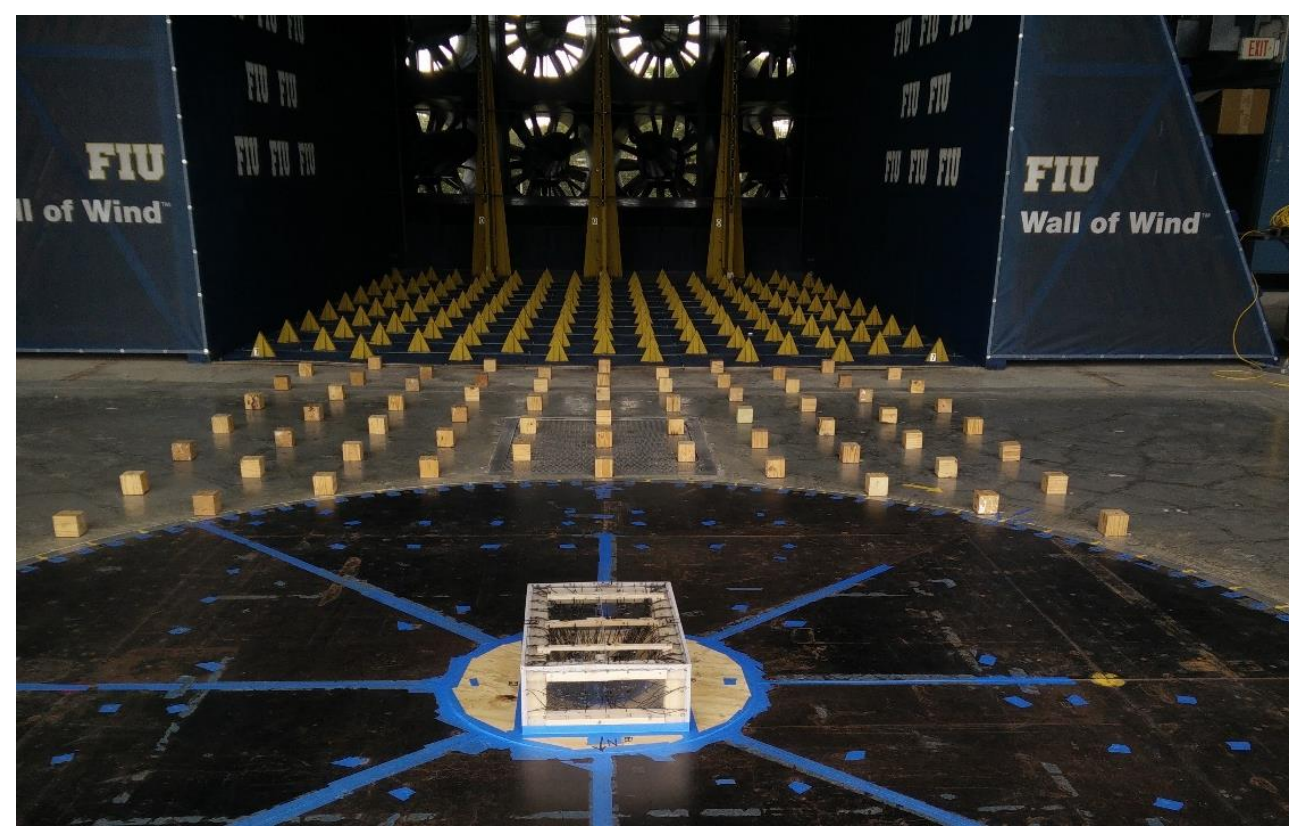

Figure 25 - Extension of roughness elements for 1:20 model

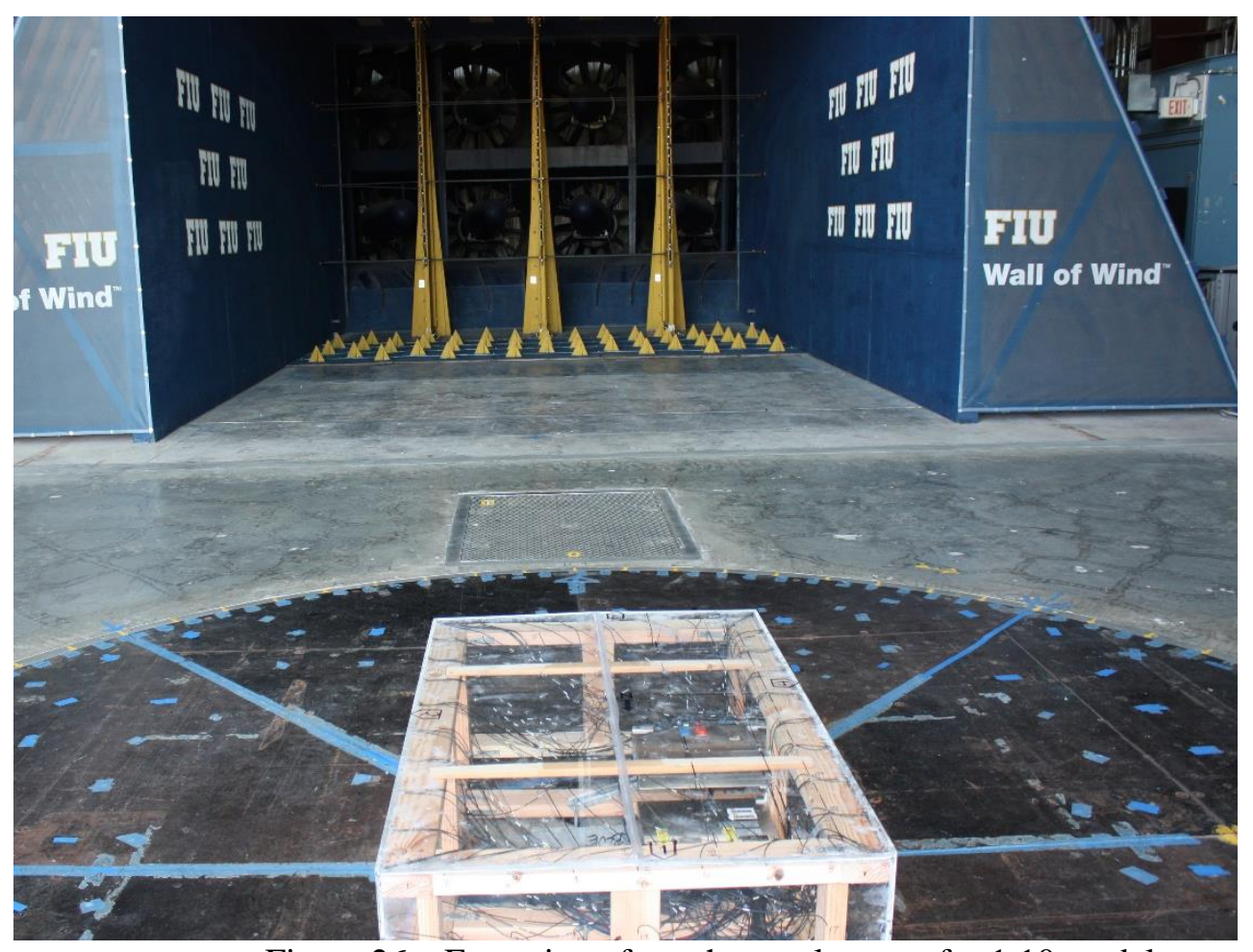

Figure 26-Extension of roughness elements for 1:10 model 


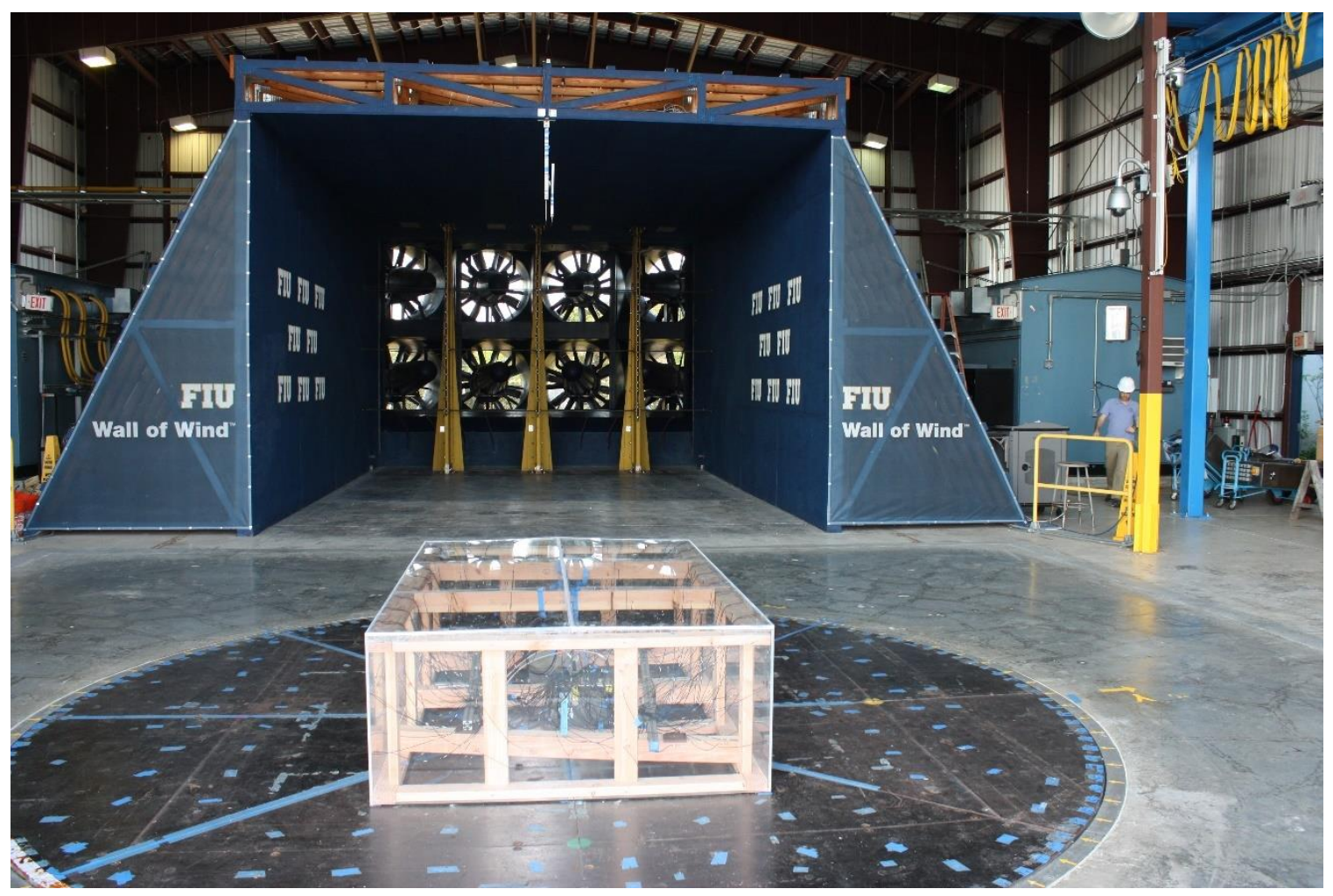

Figure 27 - Extension of roughness elements for 1:6 model

\subsection{Wind Speed and Turbulence Intensity Profiles}

Wind speeds were measured at the center of the turntable, both with Cobra probes and also pitot tubes connected to the pressure scanning system. Pitot tube measurement was done as a verification tool for mean wind speed profiles, and Cobra probe data were the basis for all wind speed and turbulence calculations. Wind speeds were collected at three fan throttles of $40 \%, 70 \%$ and $100 \%$ to provide required information needed for the Reynolds number studies. A full fan throttle of $100 \%$ is equivalent to a wind speed of approximately $71 \mathrm{~m} / \mathrm{s}$ at the reference point on the flow management box exit at the height of $4.3 \mathrm{~m}$. Table 2 shows the along wind component ( $\mathrm{u}$ as defined in Figure 10) of the mean 
wind speed and turbulence intensity obtained at each model eave height. The measured wind speed and turbulence intensity profiles are displayed in Figure 17. These profiles are derived from the free stream wind speed measurements of each terrain and are normalized to the model eave height (Figure 17) at each corresponding scale. The obtained profiles are compared to ESDU [1] profiles.

Table 3 - Measured mean wind speed and turbulence intensity at model eave heights

\begin{tabular}{|c|c|c|c|c|c|}
\hline \multirow{2}{*}{ Scale } & \multicolumn{3}{|c|}{$\begin{array}{c}\text { Umean at Eave Height } \\
(\mathbf{m p s})\end{array}$} & \multirow{2}{*}{$\begin{array}{c}\mathbf{I}_{\mathbf{u H}} \text { at eave } \\
\text { height }\end{array}$} & ${ }^{{ }^{*}} \mathbf{L}_{\mathbf{u m}} / \mathbf{b}_{\mathbf{m}}$ \\
\cline { 2 - 4 } & $\mathbf{4 0 \%}$ & $\mathbf{7 0 \%}$ & $\mathbf{1 0 0 \%}$ & & \\
\hline $\mathbf{1 : 1 0 0}$ & 13.62 & --- & 35.1 & $19.73 \%$ & 6.6 \\
\hline $\mathbf{1 : 5 0}$ & 14.8 & 25.15 & 36.6 & $19.90 \%$ & 5.1 \\
\hline $\mathbf{1 : 2 0}$ & 17.6 & --- & --- & $18.07 \%$ & 2.5 \\
\hline $\mathbf{1 : 1 0}$ & 21.74 & --- & --- & $11.00 \%$ & 1.6 \\
\hline $\mathbf{1 : 6}$ & 22.14 & 38.76 & 55.37 & $10.20 \%$ & 0.78 \\
\hline
\end{tabular}




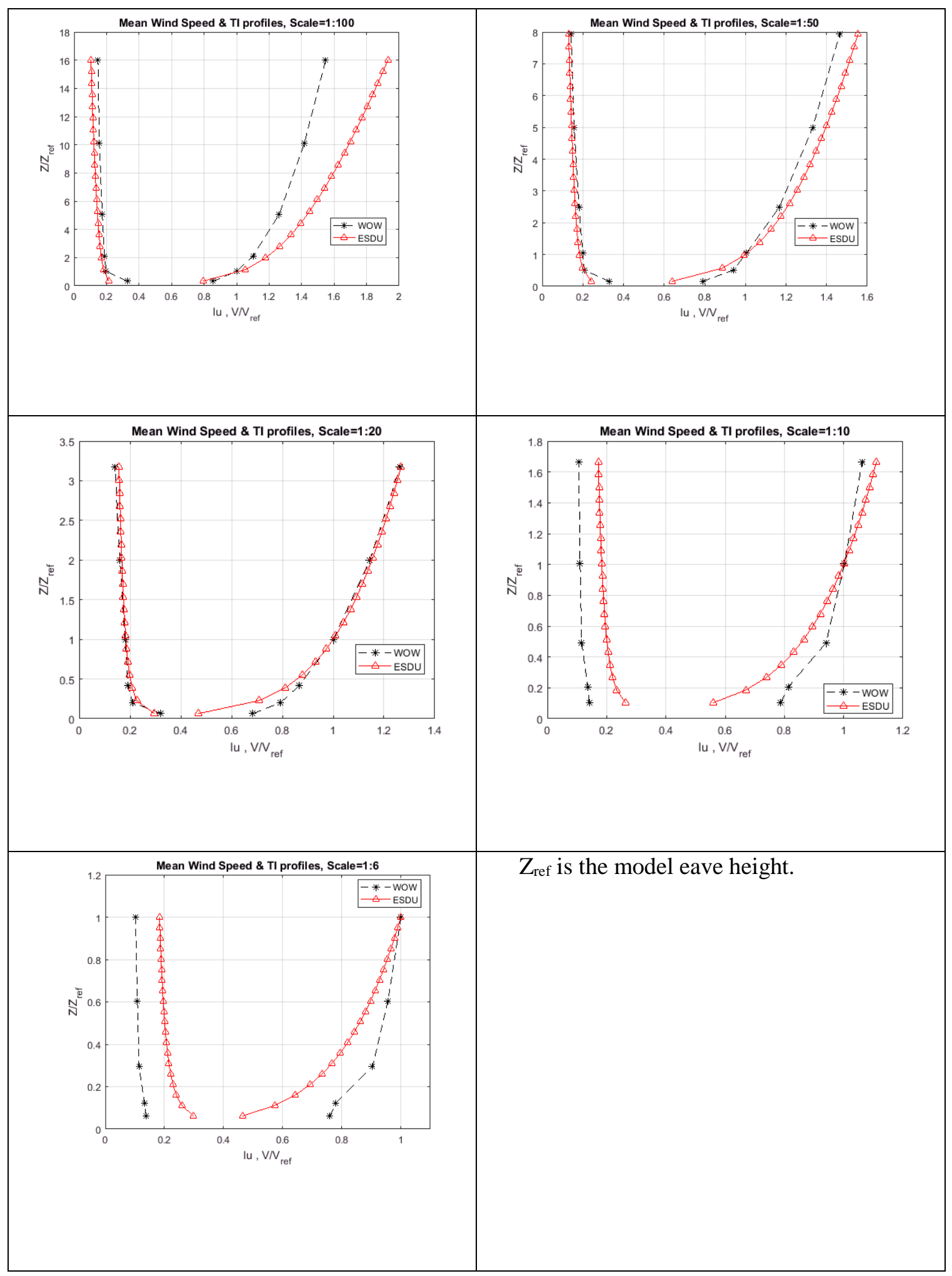

Figure 17 - Normalized Mean wind speed and turbulence intensity profiles 


\subsection{Turbulence Power Spectra}

Non-dimensional turbulence power spectra for all the simulated scales are plotted in Figure 29 in comparison with full-scale non-dimensional spectra for $\mathrm{z}_{0}=0.02 \mathrm{~m}$ and $\mathrm{z}_{0}=0.05 \mathrm{~m}$. Comparing the turbulence power spectra indicates a good match between the WOW and full scale at smaller models while moving toward larger scales, a growing discrepancy over the lower frequencies (i.e., frequencies belonging to larger eddies in the flow) is observed. The target of the flow simulation process was to match the highfrequency end of the turbulence spectrum between the model and the full scale. According to Figure 29, this goal is achieved for the targeted open terrain condition, and the concept of Partial Turbulence Simulation (PTS) is very well displayed. The effect of the missing low-frequency content of the turbulence is then analytically compensated through the PTS approach, as discussed in the subsequent sections of this document. Since the amount of the missing low-frequency turbulence is considerably lower in the first three plots, i.e., scales 1:100, 1:50 and 1:20, one should expect the effect of PTS peak correction to be smaller for these scales as compared to that for the 1:10 and 1:6 scales. 


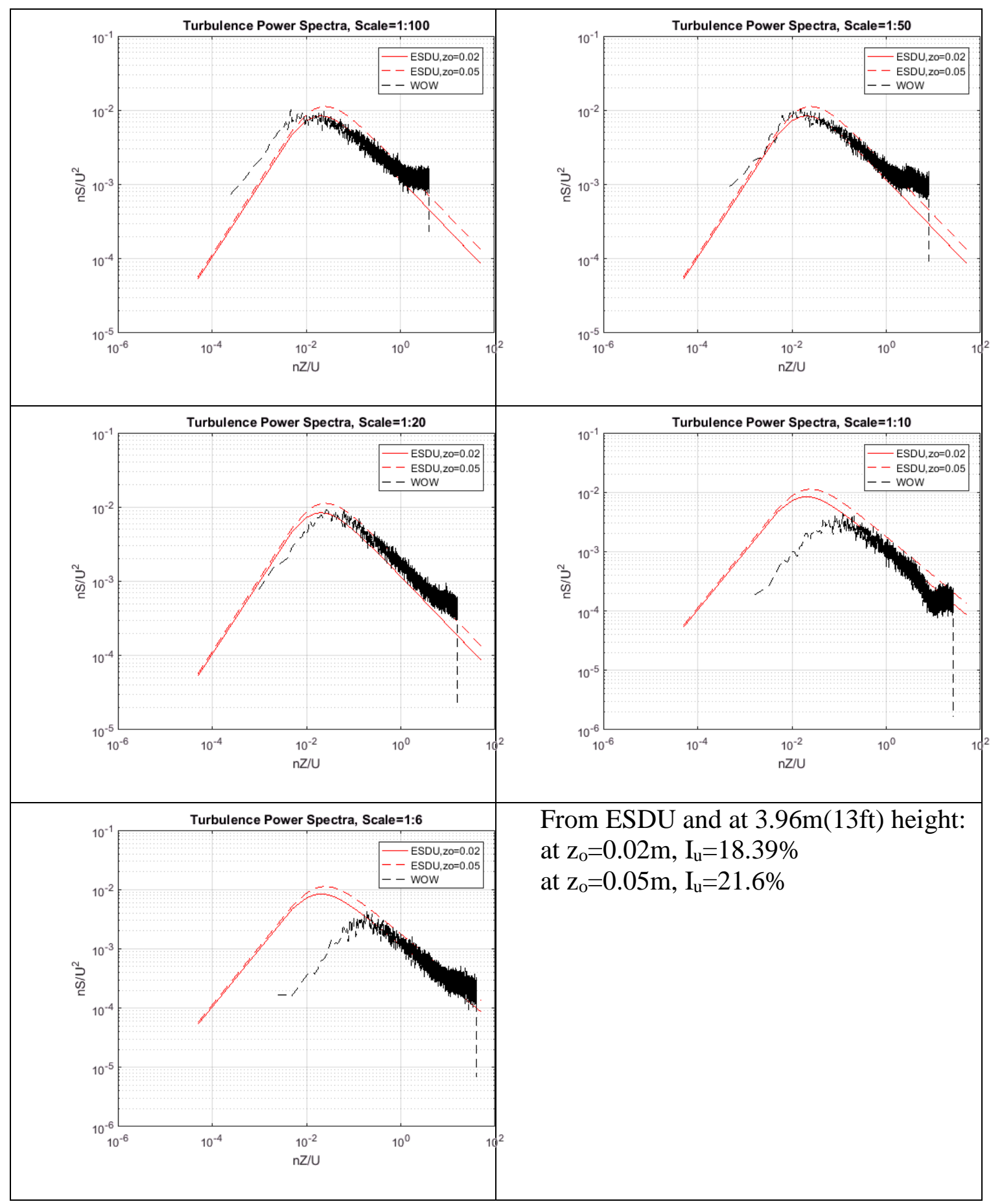

Figure 29 - Turbulence power spectra $(\mathrm{n}=$ frequency, $\mathrm{z}=$ model building height, $\mathrm{U}=$ mean wind speed at building eave height, $S=$ turbulence power spectra) 


\subsection{Time Scales and Calculation of the PTS Parameters}

The sample record length should be long enough to achieve stable statistics of the data time histories. In the full-scale, a record length of 10 minutes or more (preferably 1 hour) is known to provide such stable data, which is basically to ensure flow fluctuations are adequately captured. In order to have a sufficient record length in the model scale testing, the turbulence characteristic time can be used as a similarity parameter that reflects the large eddy turnover and is defined as ${ }^{x} L_{U} / U$. Considering the flow data from the TTU field measurements (Table 4) The characteristic time of full scale turbulence, $x L u / U$, is equal to 4.57 seconds and the 15-minute record length is equivalent to about 197 characteristic times:

$$
\frac{x L u}{U}=\frac{35 \mathrm{~m}}{7.66 \mathrm{~m} / \mathrm{s}}=4.57 \mathrm{~s}, \quad \frac{T U}{x L u}=\frac{15 \mathrm{~min} \times 60 \mathrm{~s} / \mathrm{min}}{4.57 \mathrm{~s}} \approx 197
$$

This means in the 15-minute record length, approximately 197 large eddies have advected past the building. Considering the turbulence length scales at the WOW which are in the order of $0.5 \mathrm{~m}$ and the $1: 100$ scale model case which has the lowest wind speed at the eave height, the required time duration to generate the same number of characteristic times was evaluated as 7 seconds:

$$
\frac{x L u}{U}=\frac{0.5 \mathrm{~m}}{13.62 \mathrm{~m} / \mathrm{s}}=.036 \mathrm{~s}, \quad 197 \times .029=7.2 \mathrm{~s}
$$

The sampling time of 60 seconds was selected for each of the tests which apparently exceeds the required $7 \mathrm{~s}$ time as calculated, thus it is a sufficient record length for all the cases.

Based on the definitions and the procedure described in Chapter 2, the required parameters for the PTS analysis were calculated and outlined in Table 4. These parameters 
were then used as inputs to the PTS MATLAB package. The codes and more detailed information of the model definitions are included in Appendix 0.

Table 4 - Calculations of PTS parameters

\begin{tabular}{|r|l|c|c|c|c|c|c|}
\hline No; & Parameter & $\begin{array}{c}\text { Full } \\
\text { Scale }\end{array}$ & $1: 100$ & $1: 50$ & $1: 20$ & $1: 10$ & $1: 6$ \\
\hline 1 & $I u$ & $21.60 \%$ & $19.73 \%$ & $19.90 \%$ & $18.07 \%$ & $11.00 \%$ & $10.20 \%$ \\
\hline & $I v$ & $20.7 \%$ & $11.65 \%$ & $11.45 \%$ & $12.51 \%$ & $9.65 \%$ & $9.44 \%$ \\
\hline 2 & ${ }^{x} L_{u}(m)$ & 35 & 0.262 & 0.4 & 0.5 & 0.638 & 0.52 \\
\hline 3 & $H(m)$ & 3.96 & 0.0396 & 0.0792 & 0.1980 & 0.3960 & 0.6600 \\
\hline 4 & $U(m / s)$ & 7.66 & 13.62 & 14.80 & 18.30 & 19.90 & 20.70 \\
\hline 5 & $t(s e c)$ & 900 & 60 & 60 & 60 & 60 & 60 \\
\hline 6 & $I_{u L}$ & $8.79 \%$ & $8.40 \%$ & $11.83 \%$ & $18.59 \%$ & $19.04 \%$ \\
\hline 7 & cut off $(n c)$ & 0.021 & 0.020 & 0.027 & 0.119 & 0.149 \\
\hline 8 & $t_{\text {gust }, p}$ & 21.89 & 22.46 & 16.82 & 3.80 & 3.02 \\
\hline 9 & $t_{\text {gust }, m}$ & 0.160 & 0.299 & 0.494 & 0.238 & 0.307 \\
\hline 10 & max N & 375 & 201 & 122 & 252 & 195 \\
\hline 11 & Selected N= & 100 & 100 & 100 & 100 & 100 \\
\hline 12 & $t_{\text {sub }}$ at model (s) & 0.60 & 0.60 & 0.60 & 0.60 & 0.60 \\
\hline 13 & $t_{\text {sub }}$ at full-scale (s) & 82.13 & 45.09 & 20.44 & 9.55 & 5.91 \\
\hline 14 & $G_{i}$ & 0.09126 & 0.05010 & 0.02272 & 0.01061 & 0.00656 \\
\hline
\end{tabular}




\section{CHAPTER IV}

\section{RESULTS AND DISCUSSIONS}

\subsection{Comparison of Results of the Weighted Average PTS and the 2DPTS Methods}

In the first section of this chapter, the effectiveness and accuracy of the proposed weighted average PTS method are investigated. As discussed in Chapter 2, this method is intended to simplify the process of the two-dimensional approach where instead of a $2 \mathrm{D}$ process, the simplified one-dimensional PTS is applied at a range of various directions around the central wind direction. The weighted average method is developed based on the assumption of a Gaussian distribution of the lateral component of the wind turbulence, and a weighting function is applied to the pressures corresponding to each wind direction around the central angle. This would be considered as a substitute approach to include the contribution of the lateral turbulence into the simplified one-dimensional PTS process.

The result from the application of the weighted average PTS on the roof pressure data is presented in this section. Figure 30 shows a repeated plot of the tap layout and numbering for an easier reference. Figure 31 demonstrates the comparison between the weighted average PTS $\left(\mathrm{C}_{\mathrm{p} \text { min,Avg }}\right)$ which is actually a simplified 2DPTS, the 2DPTS $\left(\mathrm{C}_{\mathrm{p} \text { min,2DPTS }}\right)$ and the full-scale results $\left(C_{p}\right.$ min,TTU $)$ for all the roof taps of the 1:6 model. The 1:6 scaled building is the largest model of this study, and one should expect to see the highest degree of PTS corrections as the amount of missing low-frequency content is the largest at this scale (Figure 29).

According to Figure 31, the weighted average method is generally producing similar results to the 2DPTS with few discrepancies. When those results are compared to those from full-scale, it is interesting to see the weighted average is making even a better estimate 
of the highest magnitudes of peaks, when compared with 2DPTS. The results from the direction of $141^{\circ}$ for the same set of taps are shown in Figure 32, where the weighted average method is compared to the simple 1DPTS $\left(\mathrm{C}_{\mathrm{p} \text { min, } \mathrm{PTS}}\right)$ and the field data. While at most points with lower magnitudes of peak pressures the two methods return similar results, but a considerable improvement resulted from the application of the weighted average method. A closer view of some of the roof taps is presented in Figure 33 and Figure 34. Also, some of the corner taps with highest peak values at the $90^{\circ}$ direction are shown in Figure 35 for the 1:10 and 1:6 scales.

Figure 36 illustrates the variation of peak results versus wind direction for the tap 194 and at five different model scales. This is a corner tap at which the highest magnitudes of peaks were obtained. This plot shows how the weighted average acts as a smoothing tool to correct for sharp and sudden changes in the estimated peak values. Also, it can be seen that at the larger scales the difference between the simple PTS (one dimensional) and the weighted average becomes more substantial as a result of the increased amount of the missing turbulence intensity both in along wind and across wind directions. While at the smaller scales (e.g. 1:100), the difference is negligible.

Considering the results presented in this section, the weighted average method can be considered as a viable substitute for the 2DPTS, with a significantly reduced degree of complexity and computational requirements. Based on these results, at most of the taps the results of the simple PTS and the weighted average method are very similar, however, at the critical locations (i.e., taps with highest magnitudes of peaks), the difference can become significant. These are the taps that fall into the separation bubble or under the conical vortices, and basically, the directional fluctuations of the approach flow play an 
important role in the generation of those high peaks. So, the effect of the lateral turbulence should not be dispensed in the process of peak estimation for those locations. It is recommended that the weighted average method to be used at least for these two cases: first, in the case of perpendicular wind at which the most severe flow separation occurs at the windward edge of the roof, second in the case of quartering winds which form the conical vortices. Since the wind directions establishing those vortices are mainly in the range $20^{\circ}-65^{\circ}[60]$ (or $115^{\circ}-155^{\circ}$ when $135^{\circ}$ is the central angle in the range), the application of the weighted average method is advisable to estimate the corresponding peak values. More detailed full-scale comparisons at a broader range of taps and model scales are presented in the subsequent sections. 
Connected taps, Dir=0,SCALE 1:100
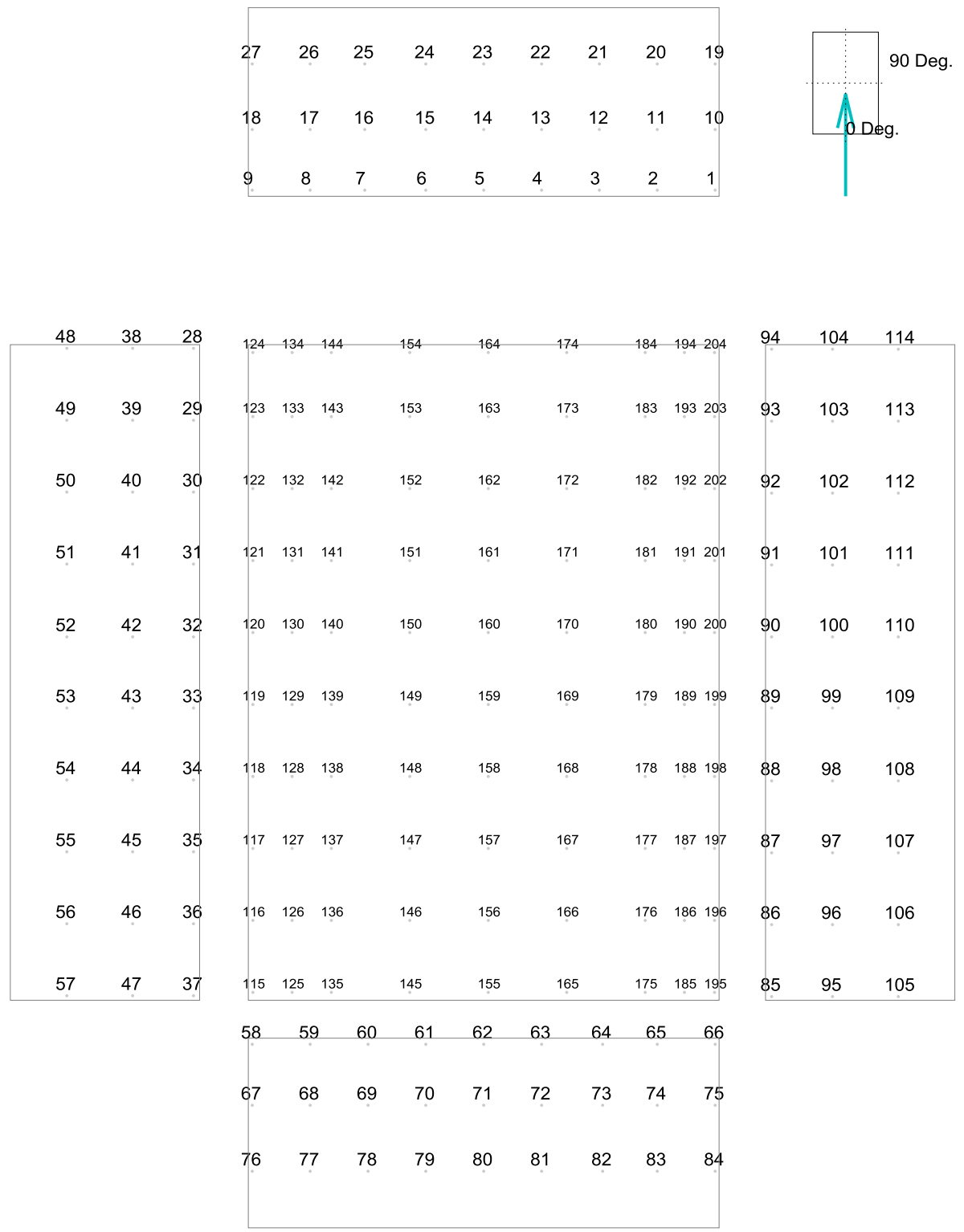

Figure 30 - Tap layout and location map (repeated) 


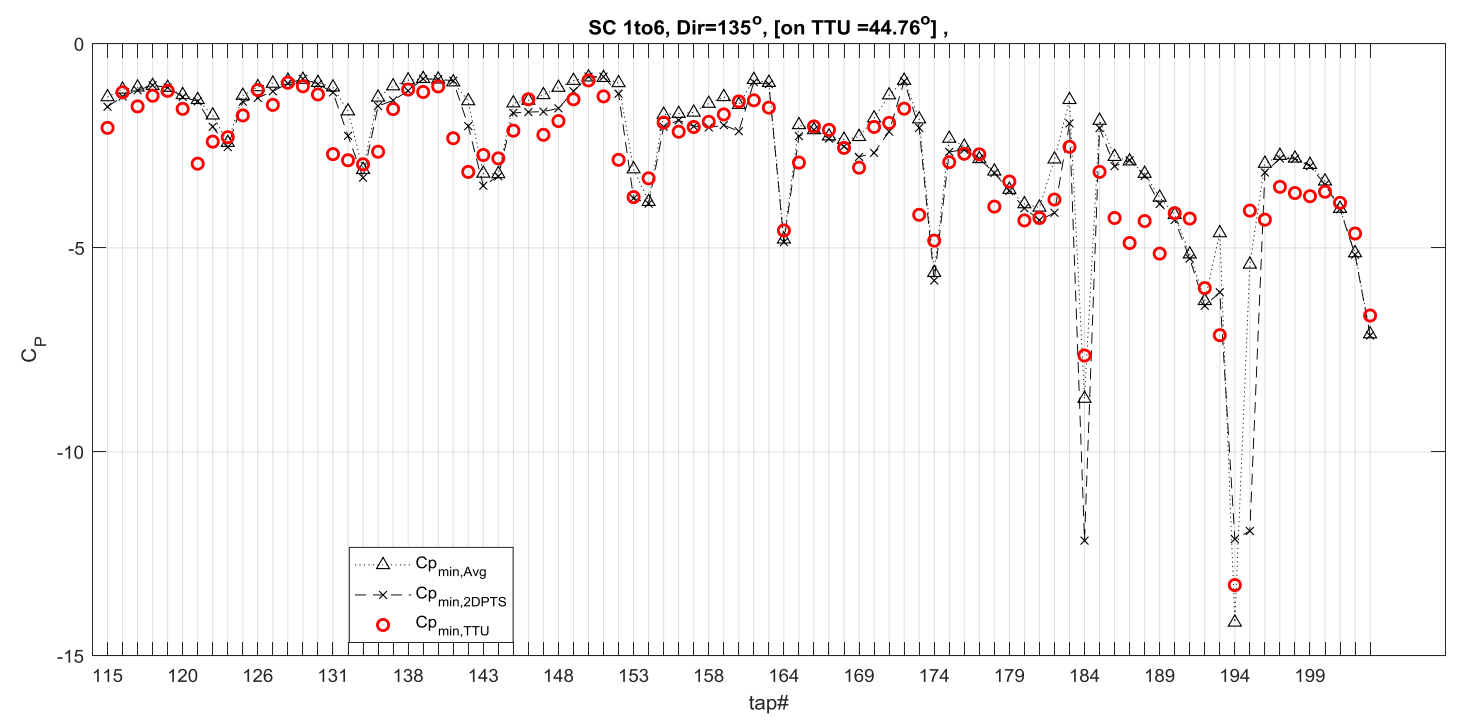

Figure $31-$ Evaluation of the weighted average PTS at roof taps, Dir=135

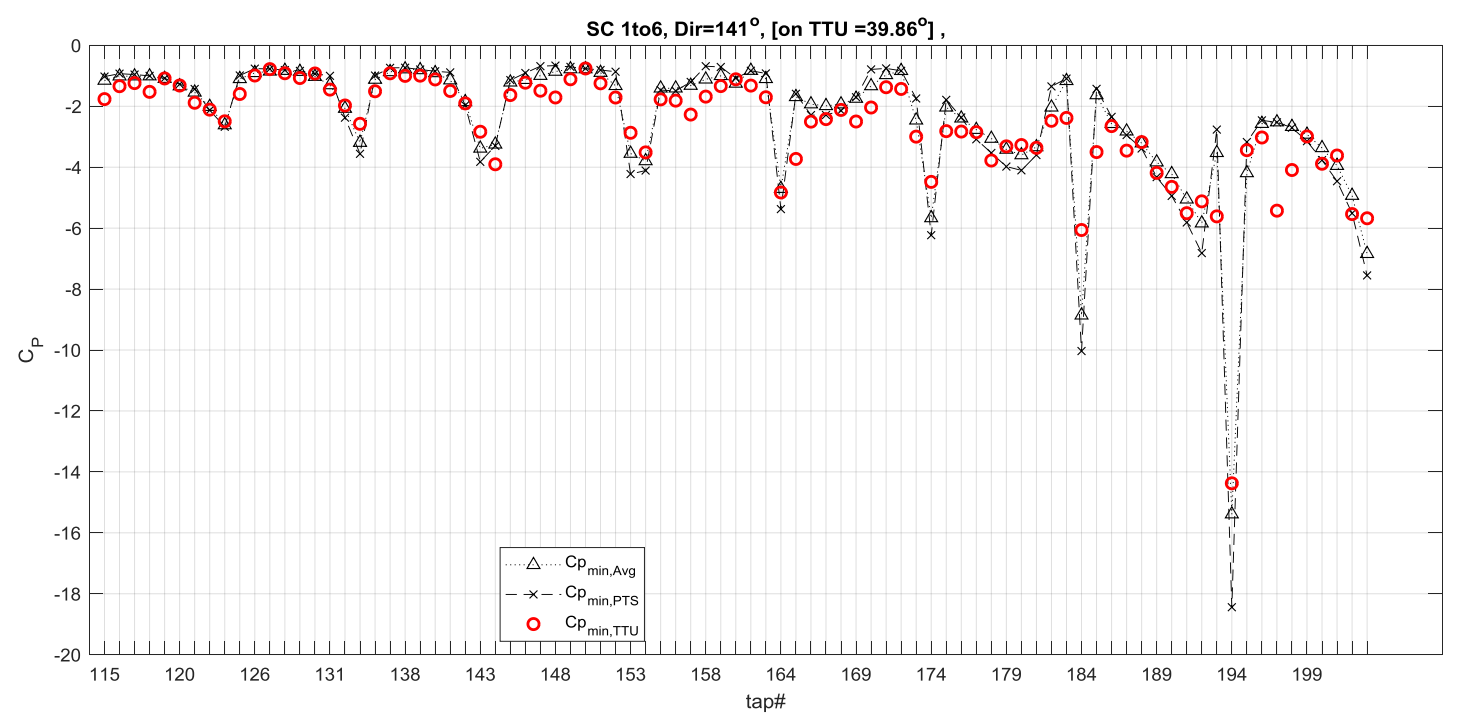

Figure $32-$ Evaluation of the weighted average PTS at roof taps, Dir=141 ${ }^{\circ}$ 


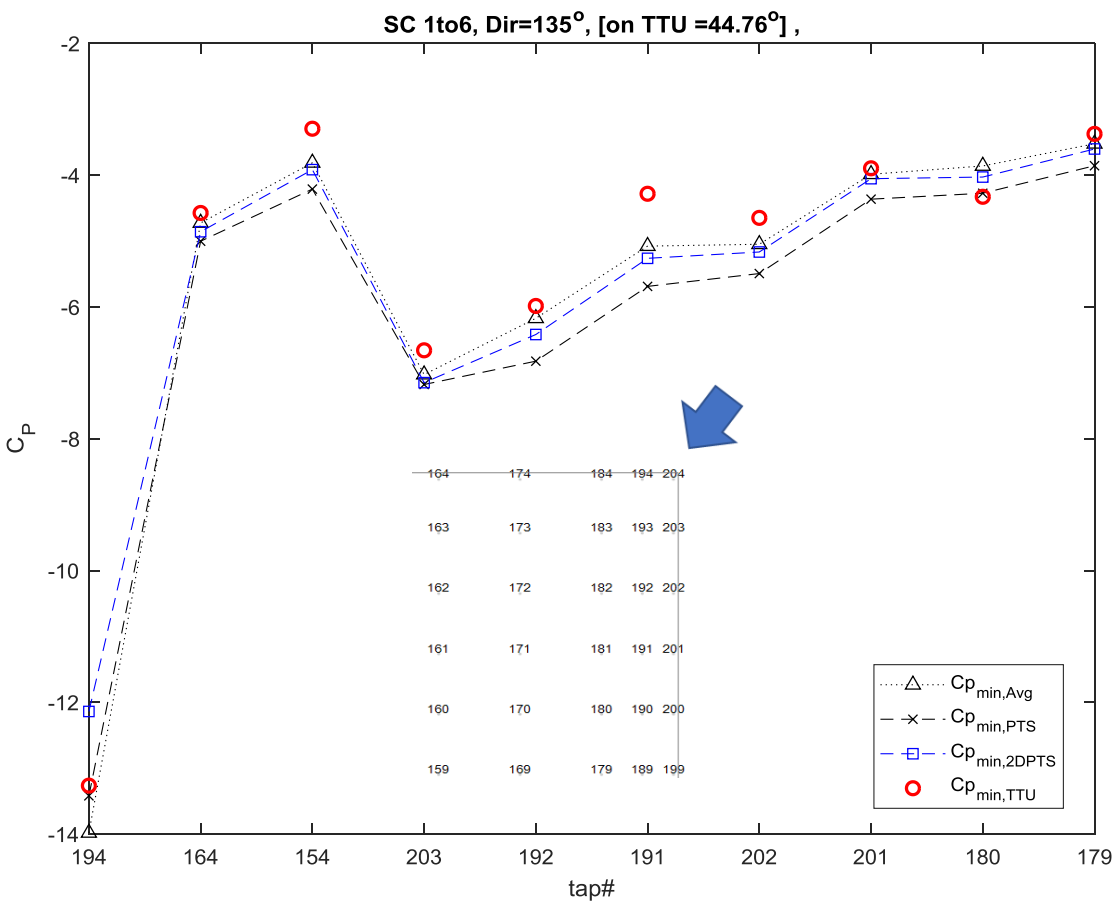

Figure $33-C_{p \min , A v g}$ at a group of roof taps, Dir=135

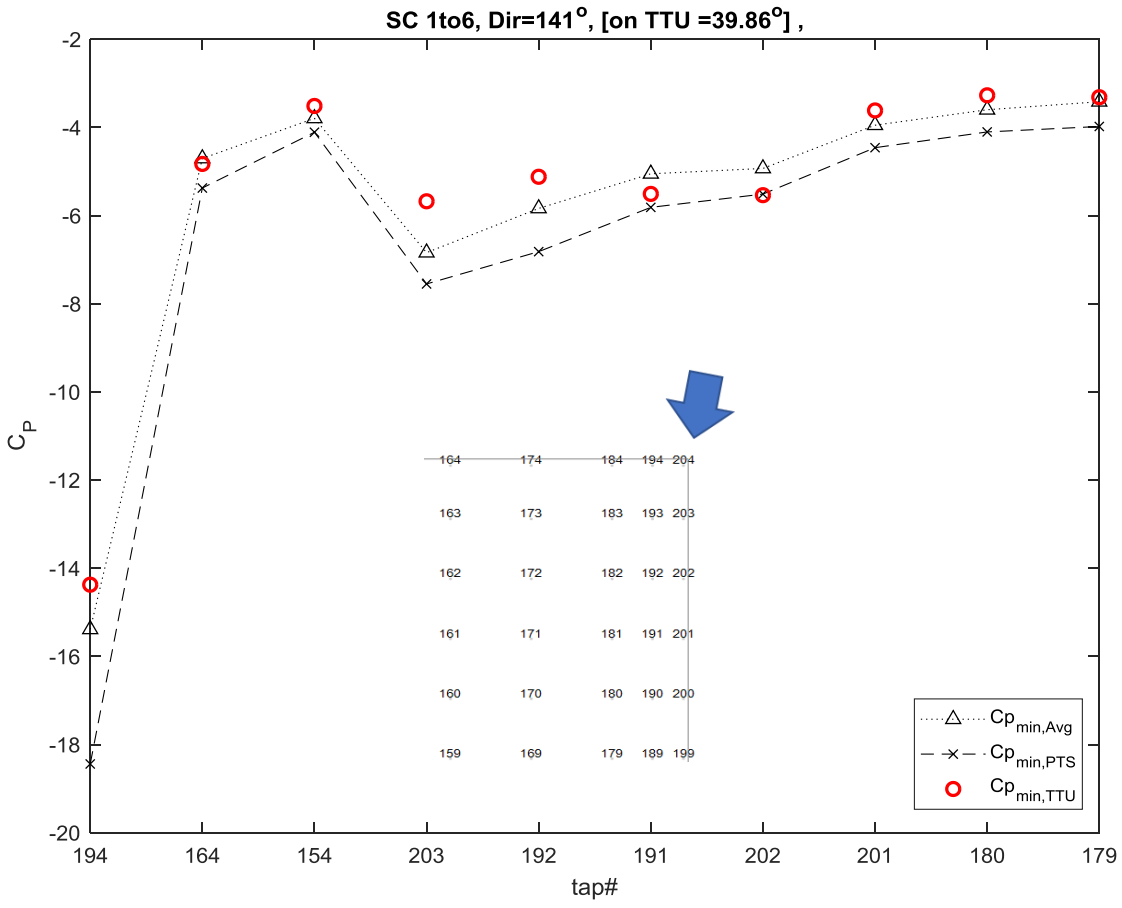

Figure $34-C_{p}$ min,Avg at a group of roof taps, Dir $=141^{\circ}$ 

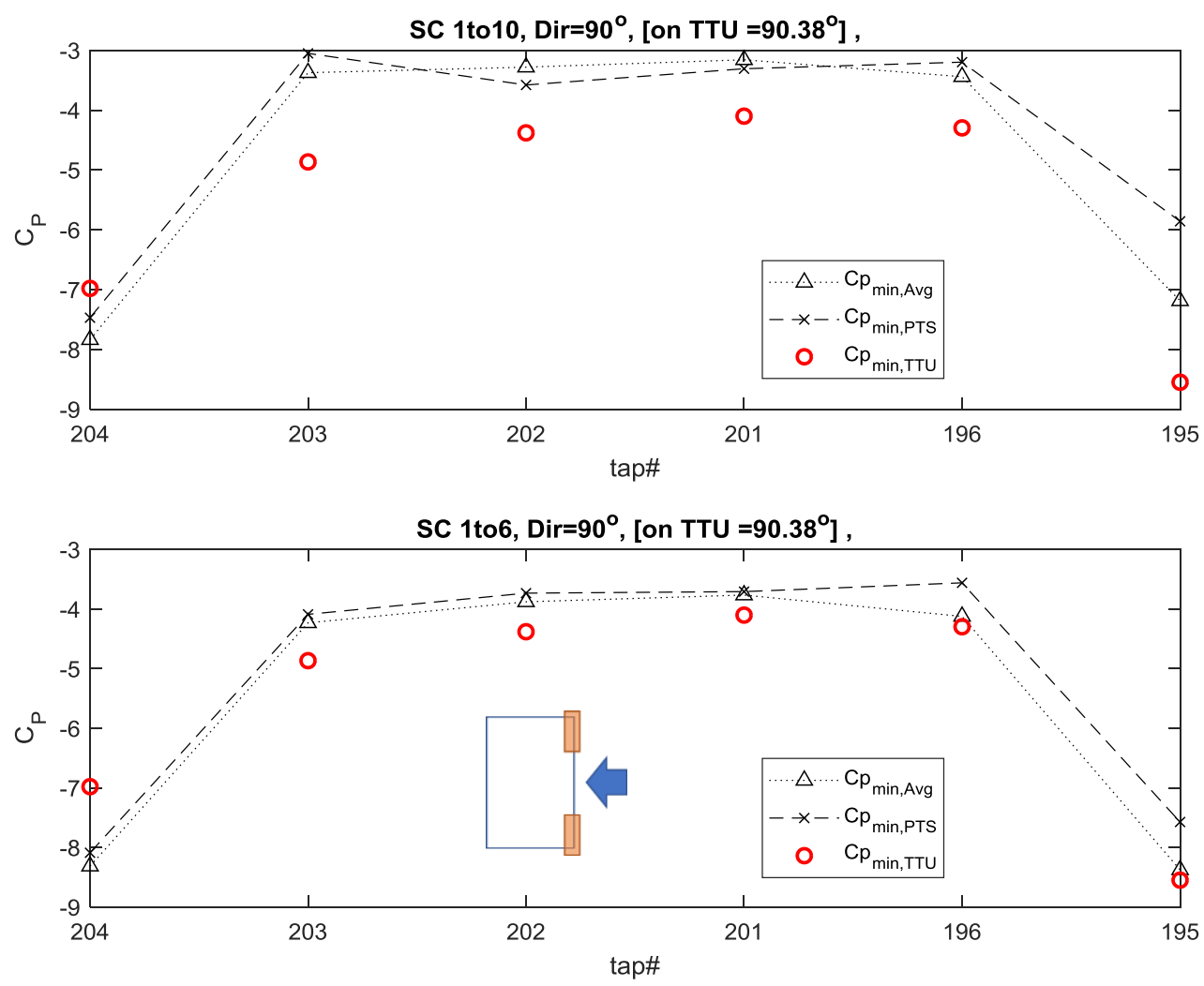

Figure $35-\mathrm{C}_{\mathrm{p} \text { min,Avg }}$ at a group of roof taps, Dir $=90^{\circ}$
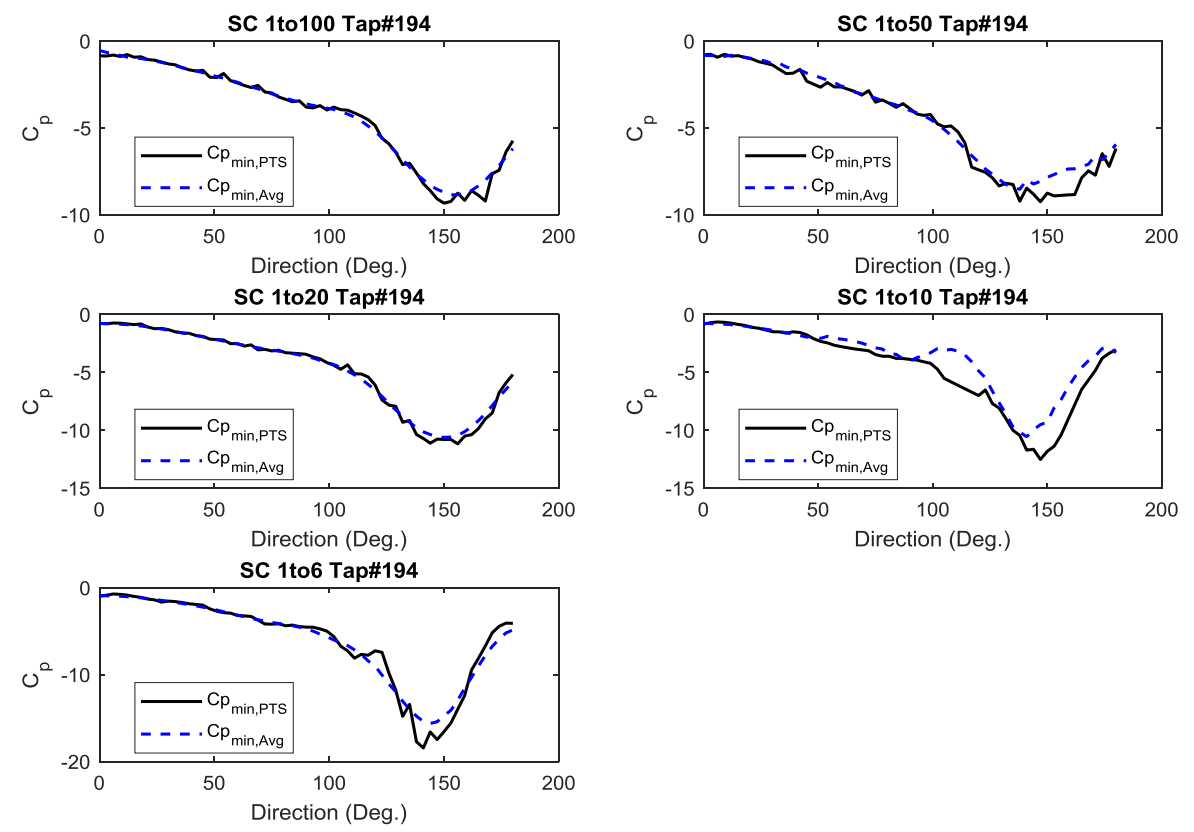

Figure $36-C_{p \text { min,Avg }} \& C_{p}$ min,PTs vs. direction, tap\#194 


\subsection{Comparison of Experimental Results to the Field Data}

\section{Mean and Peak Pressure Coefficients}

The obtained mean and peak results from the experiments are reported in this section and are compared with the field data from TTU building and other available relevant data.

Figure 38 to Figure 42 show the contours of the mean pressure coefficients $\left(C_{p}\right.$ mean $)$ on the 5 tested models followed by the full-scale results (field data) as displayed in Figure 43. The overall trend indicates a good comparison between the test results and field data. At the roof corner of the 1:100 model a slightly different pattern is observed compared to other scales and the full-scale which is because of a relatively lower suction obtained at the tap 204 which is on the corner. However, the results from the adjacent taps which are the points with the highest suctions on the full-scale, are showing reasonable results. Considering the $\mathrm{C}_{\mathrm{p}}$ values on the walls, the Wall of Wind results show somewhat higher results than those of the full-scale. This difference will be discussed further in the following sections. A tap-wise plot as shown in Figure 44 (1:6 scale) and Figure 45 (field data) helps for a side by side comparison. The highest roof suction is occurring at the corner tap 194 (see Figure 30 for the location of the tap) for which the 1:6 model is showing a $\mathrm{C}_{\mathrm{p}}=-3.86$ compared to the full-scale value of $\mathrm{C}_{\mathrm{p}}=-3.10$. In addition to these, a selected group of taps is examined more closely from various aspects. Figure 52 shows the values of the mean $C_{p}$ on the mid-wall taps (i.e., taps in the mid-height of the walls) resulted from a cornering wind at direction $135^{\circ}$. Figure 53 shows the similar plot for the direction $171^{\circ}$ which is the closest to the $180^{\circ}$, for which the full-scale data were available. According to these plots,

the model scale results are slightly higher than field data at several points. The same trend is observed as the direction $90^{\circ}$ is examined in Figure 54 where the results from a vertical 
line of taps are shown. However, the obtained results compare well with the results obtained by other researchers on the scaled models of TTU building and Silsoe cube, and also with the field data from other corresponding directions. For instance, the highest value for $\mathrm{C}_{\mathrm{p}}$,mean on the windward wall of the 1:6 model is 0.8 (Figure 54) which is close to the 1:10 model and also full-scale values reported in [13] which are in range of 0.7-0.75 for the windward face $\left(270^{\circ}\right.$ in Figure 55). Also, the similarity of results is observable by comparing Figure 54 to Figure 56, which shows the mean pressure coefficients on the Silsoe cube and the models from other wind tunnels [20], [21]. According to Figure 56, the wall facing the wind is experiencing $\mathrm{C}_{\mathrm{p}}$ values as high as $0.8-0.9$, and the roof taps are showing values about -1.2; from the WOW experiments the largest model which has been the 1:6 scale is showing the most similar results to these values.

The results from roof taps are shown in Figure 57 (for the direction $171^{\circ}$ ) and Figure 58 (for the direction $135^{\circ}$ ) where a reasonable agreement between model and field results is evident, especially for the larger models. The horizontal axis shows the location of tap through a normalized coordinate system where the y-coordinate of the taps are normalized with respect to the building height. The location and direction of the reference coordinate system as well as the schematic location of the selected line of taps is shown on the bottom corner of each plot.

The peak pressure coefficients obtained from the experiments are presented now and compared to the field data. In the first step, the variation of peak pressure coefficients with respect to the wind direction is studied for two taps near the roof edge. These are the taps which were found to have the highest negative peaks. Figure 59 shows the results for tap 203 where the $C_{p}$ min,PTS results from all model scales are displayed and compared to full- 
scale TTU data over a range of wind directions from $90^{\circ}$ to $180^{\circ}$. The plot shows that while PTS-based results from most of the models matched the lower peak pressure coefficients obtained from the field data, only the PTS-based results for the largest model (1:6) could reasonably match the highest peak coefficients obtained for the field data (between $120^{\circ}$ to $140^{\circ}$ ). The 1:6 scale model shows an overall good agreement with a $12 \%$ difference at the highest peak. In Figure 60, the peak pressure coefficients of the scale models and the full-scale are compared at tap 194 which indicates a reasonable agreement between the results of the 1:6 model (largest model) and those of full-scale. The 1:6 model results matched quite well with the very high negative pressure coefficients, such as -18 obtained from the field data. These are the critical values that would influence the wind design.

Through the next series of results, several groups of pressure taps are studied at various wind directions. To be inclusive and yet brief, the following wind directions were selected to display the detailed results: $135^{\circ}$ (to have the quartering wind), $171^{\circ}$ (the closest to the $180^{\circ}$ based on the available full-scale dataset), $35^{\circ}$ (as a direction with high peaks) and $90^{\circ}$. The plots are presented on a normalized coordinates basis where the $\mathrm{x}$ or $\mathrm{y}$ coordinates of each tap is normalized with respect to the building height $(\mathrm{H})$. The convention for the used coordinate system is displayed on each plot. Figure 61 to Figure 64 show the results from the wind direction of $45^{\circ}$. Both the PTS peak negative pressure coefficients $\left(\mathrm{C}_{\mathrm{p} \text { min,PTS})}\right.$ and weighted averaged peak negative (denoted by $\mathrm{C}_{\mathrm{p} \text { min,Avg }}$ ) are compared to the full-scale peak negative pressure coefficients. To cover a wide range of roof taps, several $\mathrm{x} / \mathrm{H}$ and $\mathrm{y} / \mathrm{H}$ values are selected to display the results. For instance, the $\mathrm{x} / \mathrm{H}=0.02$ belongs to the first line of taps parallel to the longer edge of the building (taps 204 to 195, Figure 30). According to these figures, the comparison of the results with the 
full-scale data improves as the scale is increased. Looking at the highest value which is $C_{p}=-6.7$ at full-scale, almost an exact match is obtained at 1:6. This trend is repeated at other examined locations, i.e., various $\mathrm{x} / \mathrm{H}$ and $\mathrm{y} / \mathrm{H}$ values. Figure 65 and Figure 66 show the results for the wind direction of $35^{\circ}$. The highest peak $C_{p}$ value of -13.27 is highly underestimated for models 1:100 and 1:50, and the comparisons improved for models 1:20 and 1:10. For the 1:6 model the estimated value closely matched (slightly overestimated) with its field counterpart. Considering the direction of $11^{\circ}$ at which the wall on the short side of the building is facing the wind (Figure 67, Figure 68), the results again show a good comparison between the PTS peaks and those of full scale. Figure 69 displays the results for the taps on the midline of the roof at $y / H=0.19$. As observed in this figure, the leadingedge taps show some discrepancies.

From a design perspective, it is essential to determine the worst load case. To find the worst case, the minimum $\mathrm{C}_{\mathrm{p} \text { min }}$ out of all directions was extracted for each pressure tap from a representative region of the roof (a quarter area of the roof as shaded in Figure 70). The results from all scales are shown in Figure 70 and Figure 71 for the $C_{p}$ min,Avg and the $\mathrm{C}_{\mathrm{p} \text { min,PTS }}$, respectively. As observed in these plots, the worst case of the weighted average peak is $C p=-15.6$ while the PTS peaks show a minimum value of $C_{p}=-18.4$. These should be compared to the full-scale value of $C_{p}=-17.6$ which show almost a perfect match for the 1DPTS and a 12\% difference for the weighted average PTS.

Figure 72 displays a comparison of the results with the NIST (National Institute of Standards and Technology) datasets which are produced in collaboration with the boundary layer wind tunnel at the University of Western Ontario (UWO) and include a huge database of small-scale wind tunnel experiments on several buildings of different dimensions and 
roof slopes [61], [62]. The selected dataset belongs to a 1:100 model scale of the TTU building. The NIST data correspond to two different roughness lengths: $\mathrm{z}_{0}=0.01 \mathrm{~m}$ and $\mathrm{Z}_{\mathrm{o}}=0.087 \mathrm{~m}$ as marked by NIST -1 and NIST- 2 respectively. According to this figure, the Wall of Wind results for the smaller scale models generally fall within the range of NIST results for most of roof taps. But both NIST and the small-scale results are significantly different from the highest suctions observed at full-scale. However, the results from the larger scale models (e.g. 1:10 \& 1:6) become closer to the full-scale $C_{p}$ values and as observed in this figure, the 1:6 model is showing an excellent match at the tap 194 which has the highest suction among all the roof taps. At other locations the results are somewhat conservative. It should be noted that the full-scale results are simply the observed peaks without application of any extreme value analysis to estimate the peak wind pressures.

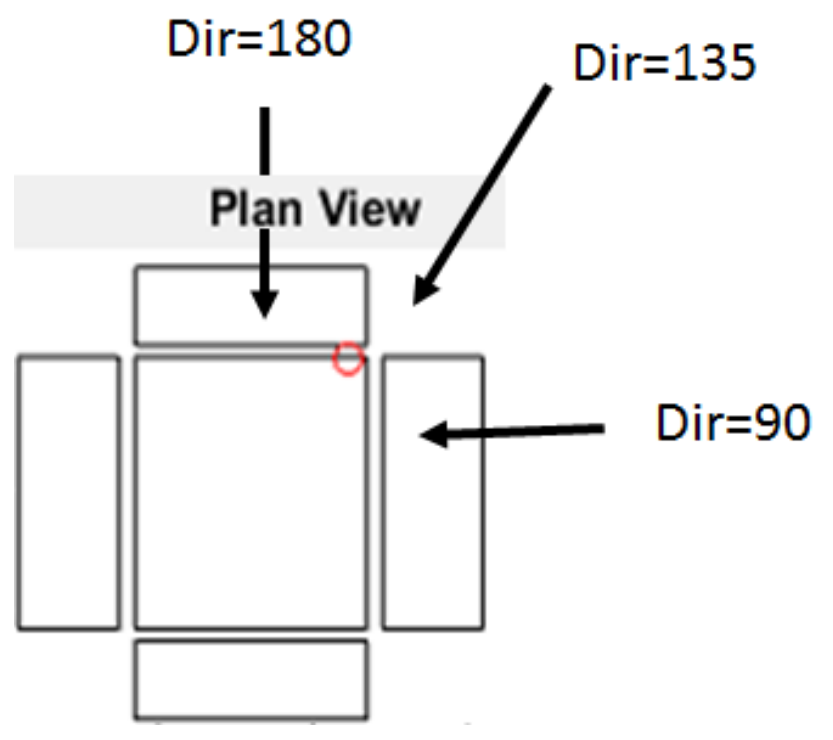

Figure 37 - Definition of wind directions 


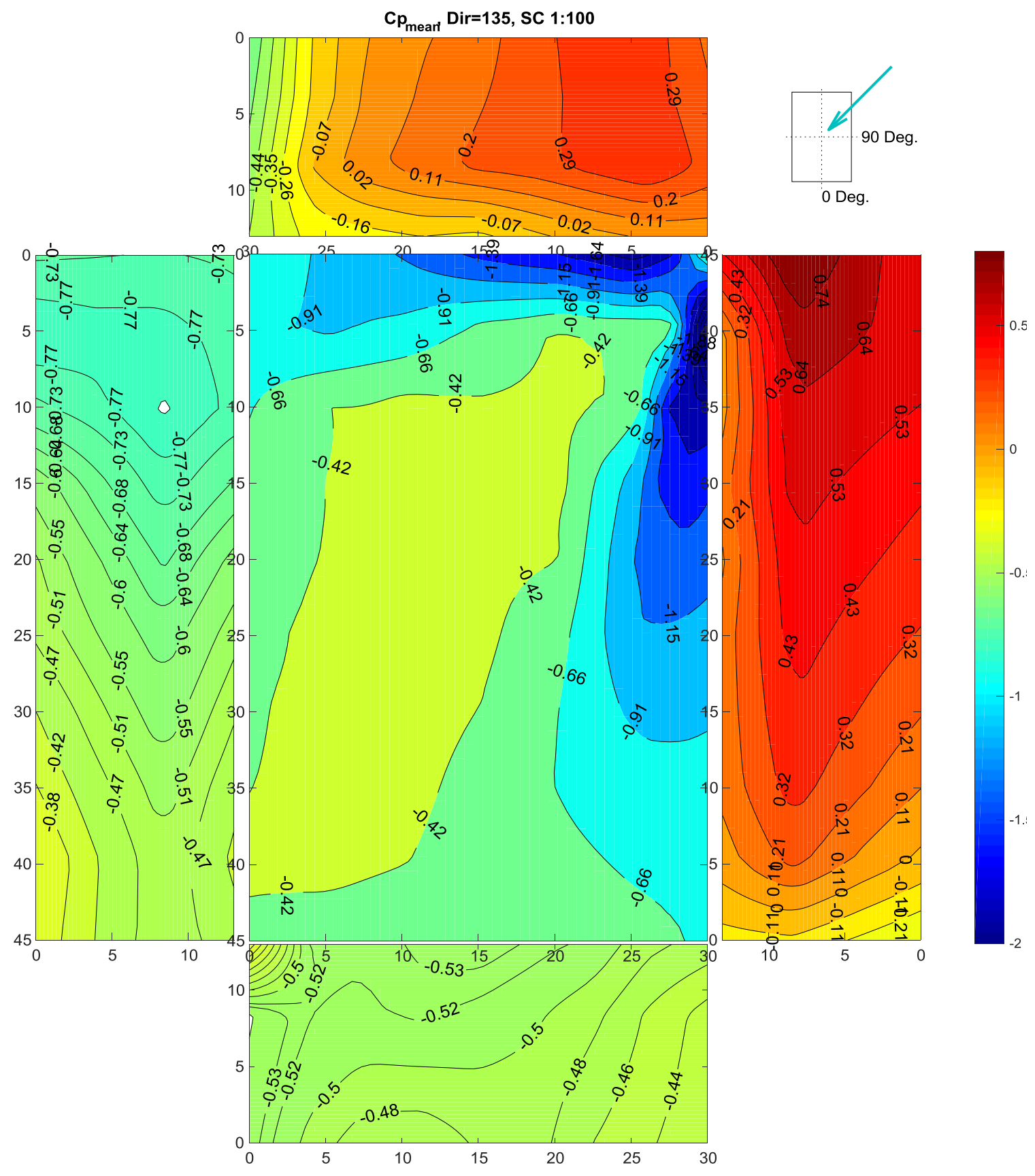

Figure $38-$ Mean $C_{p}$ distribution at 1:100 scale, Dir=135 


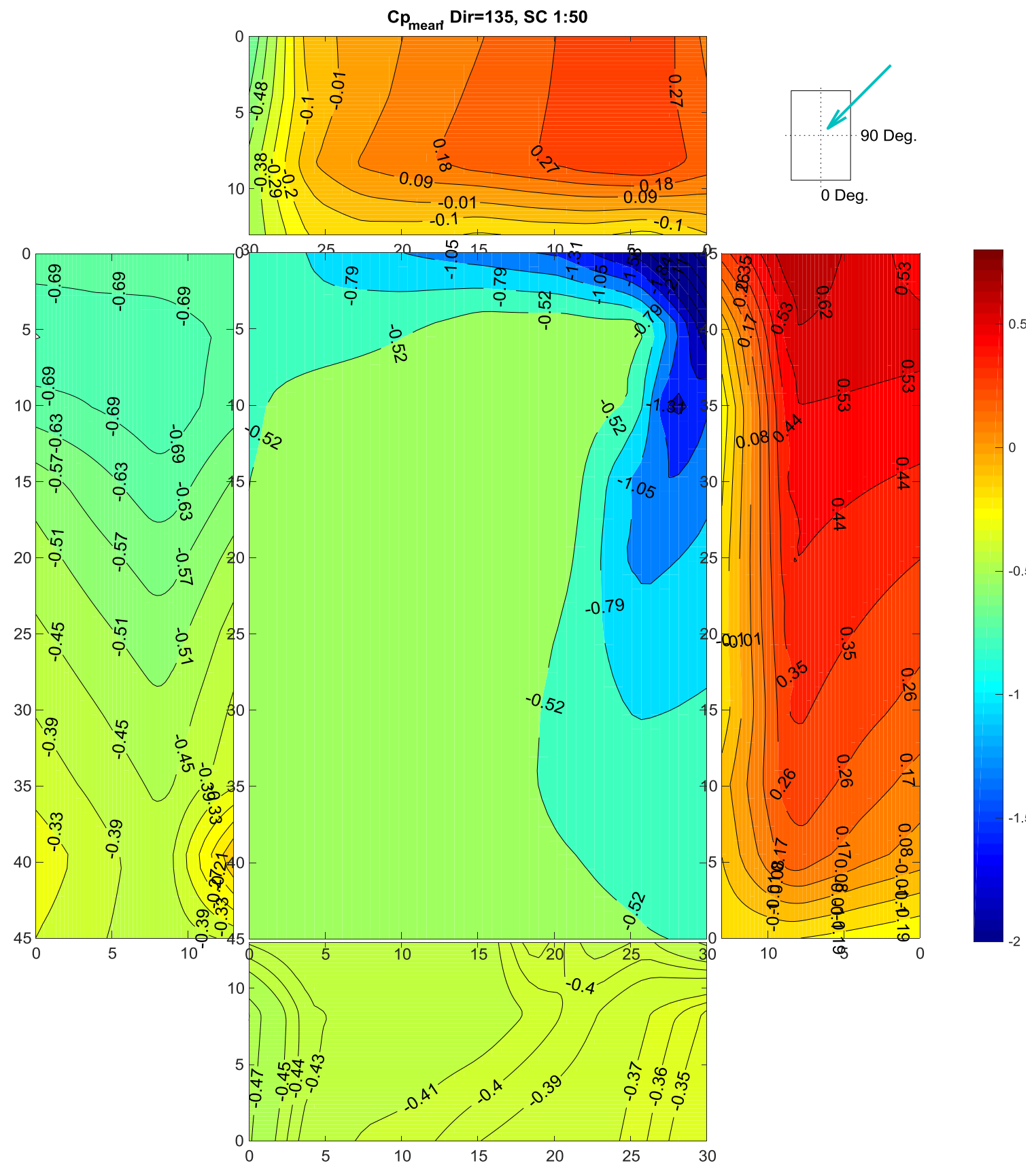

Figure $39-$ Mean $C_{p}$ distribution at 1:50 scale, Dir $=135^{\circ}$ 


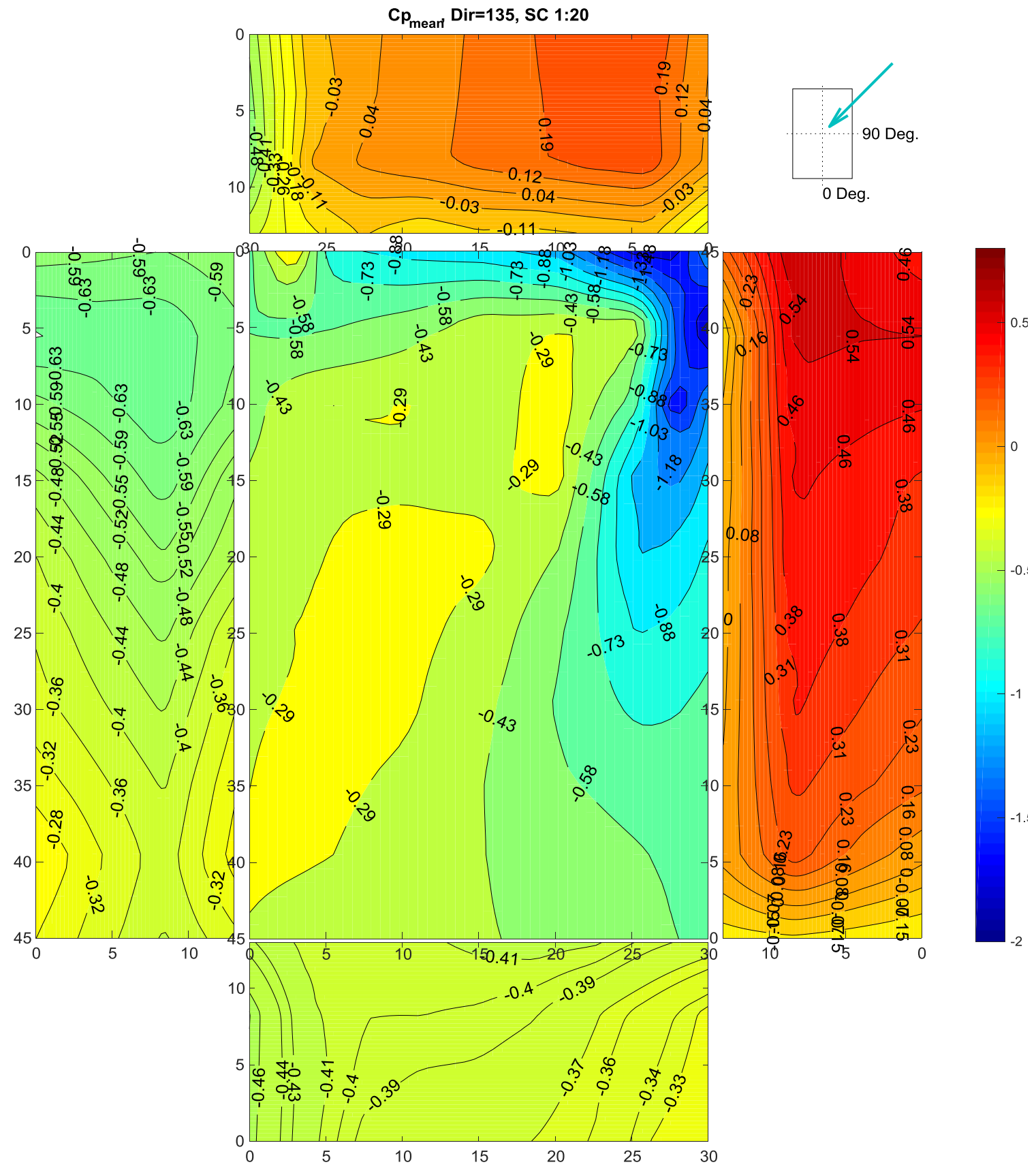

Figure $40-$ Mean $C_{p}$ distribution at 1:20 scale, $\operatorname{Dir}=135^{\circ}$ 


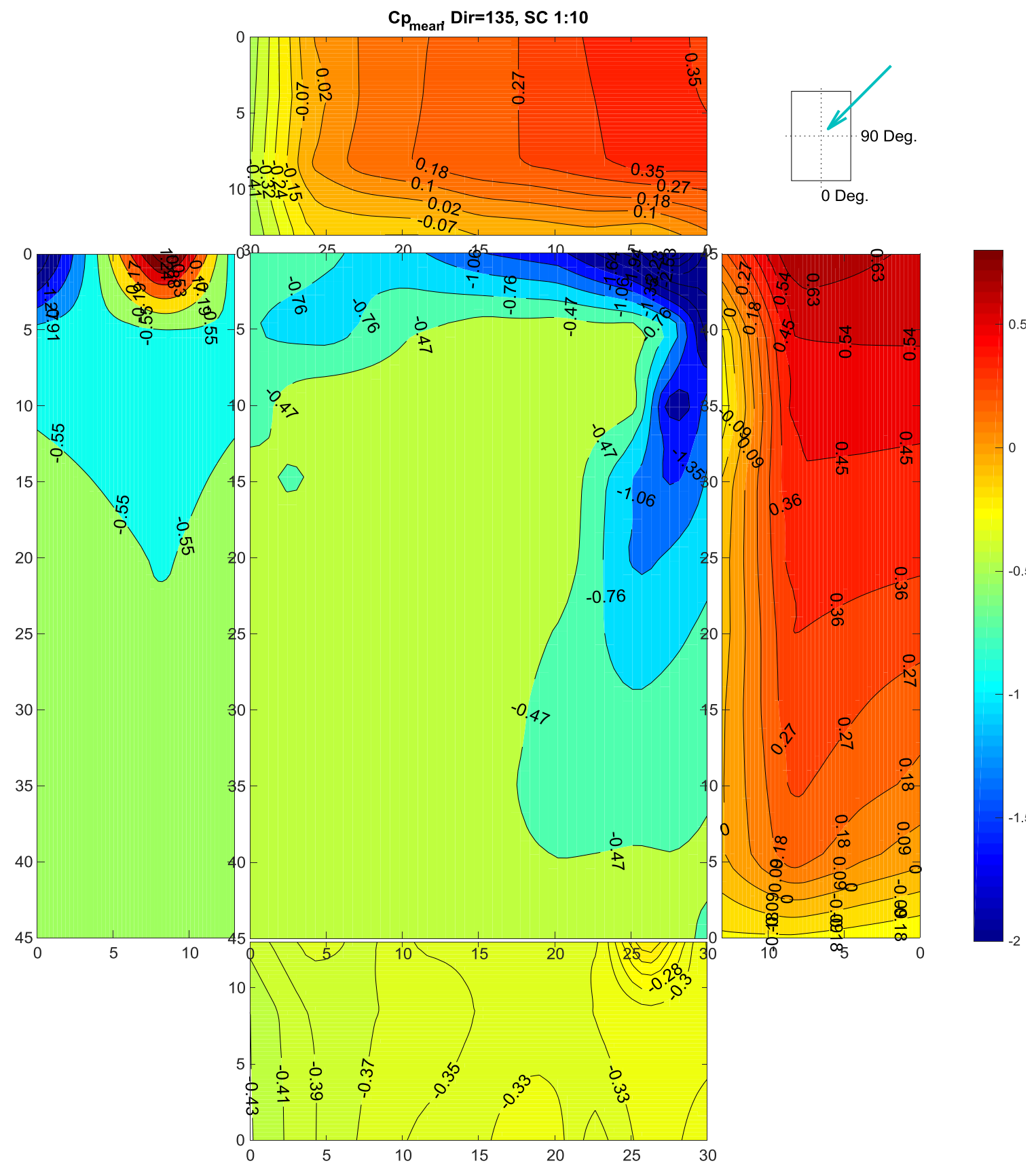

Figure $41-$ Mean $C_{p}$ distribution at 1:10 scale, Dir $=135^{\circ}$ 


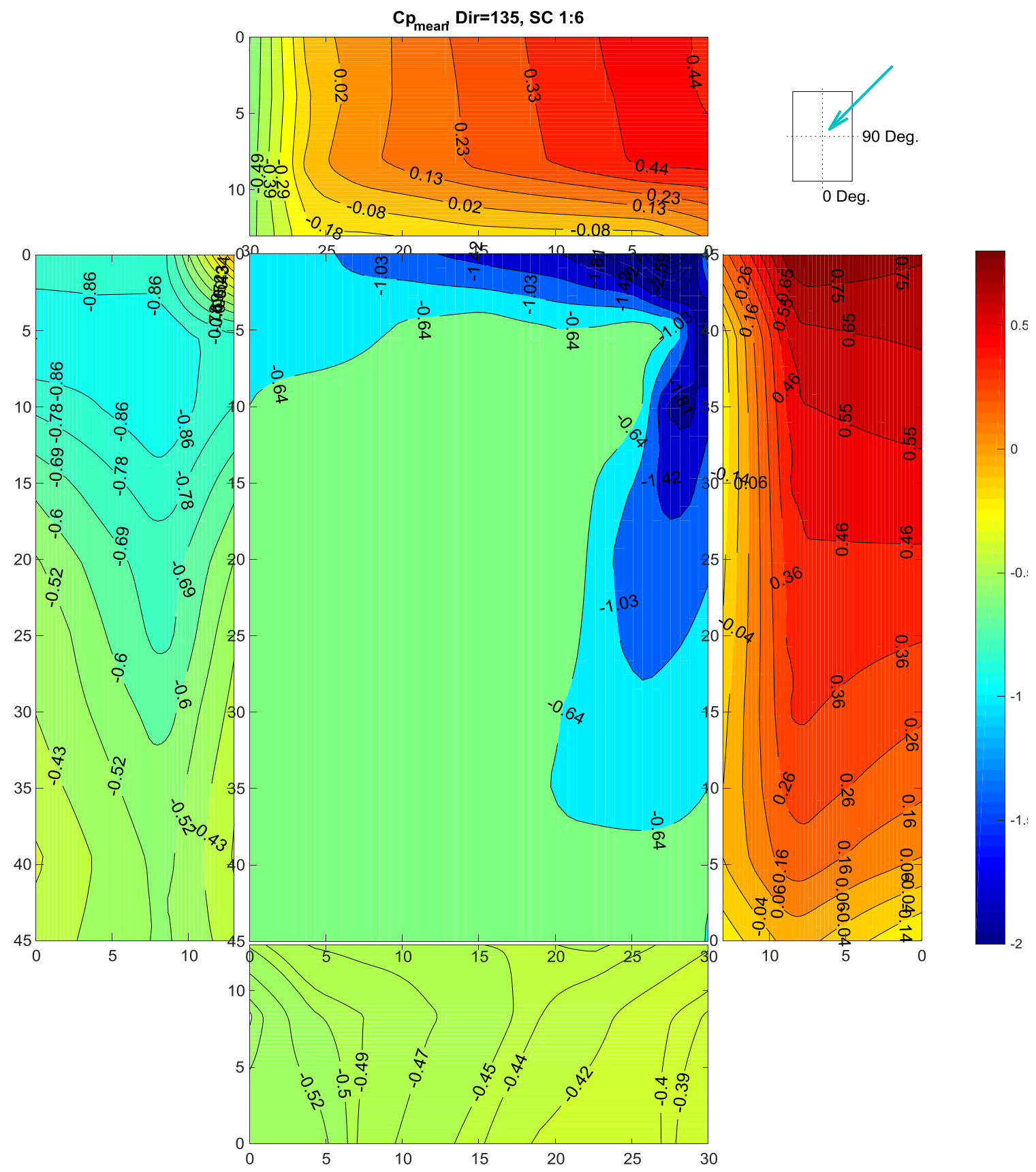

Figure $42-$ Mean $\mathrm{C}_{\mathrm{p}}$ distribution at 1:6 scale, Dir $=135^{\circ}$ 


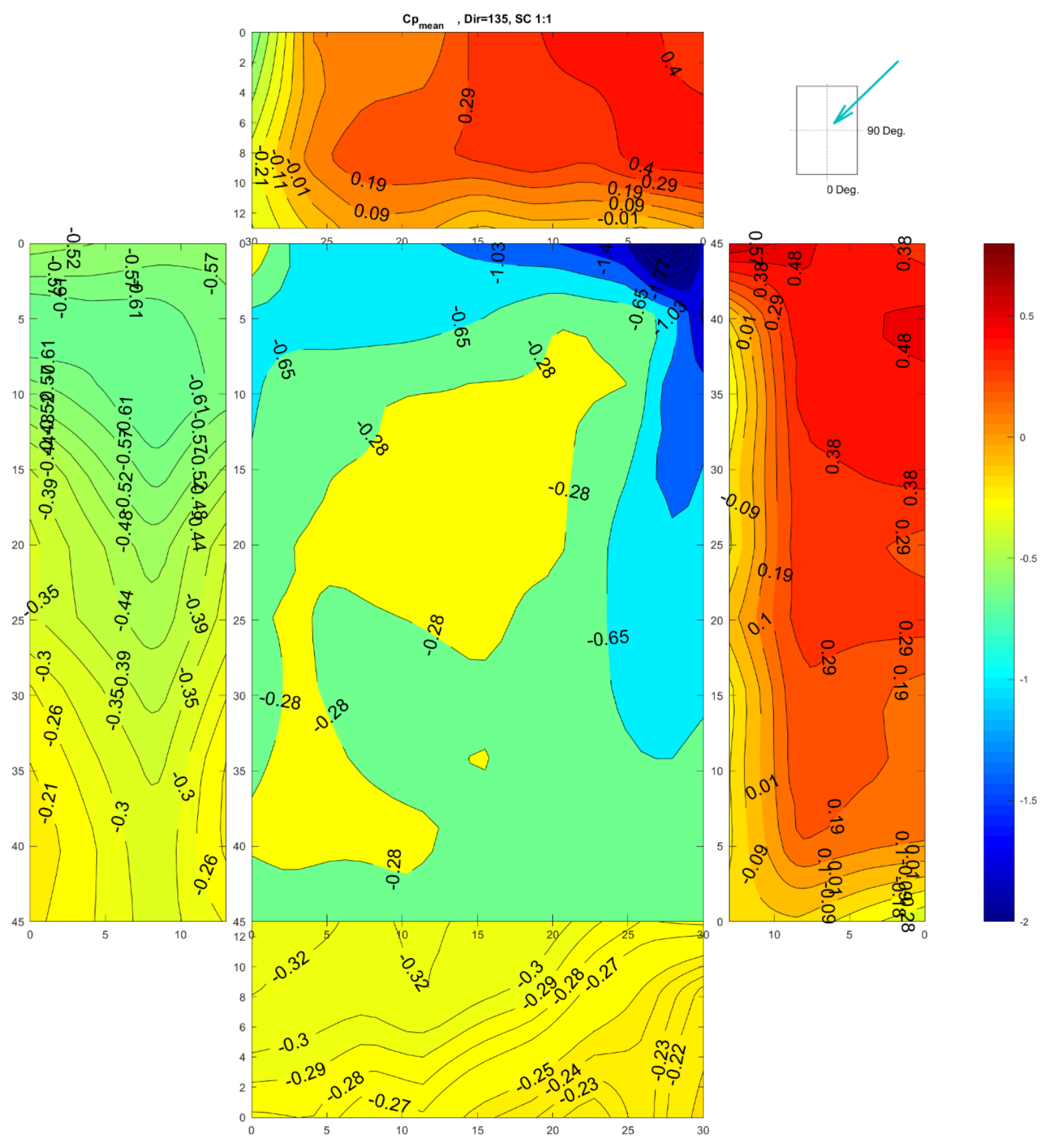

Figure $43-$ Mean $\mathrm{C}_{\mathrm{p}}$ distribution at full-scale, Dir=135 
$C p_{\text {mear }}$ Dir=135,SC 1:6
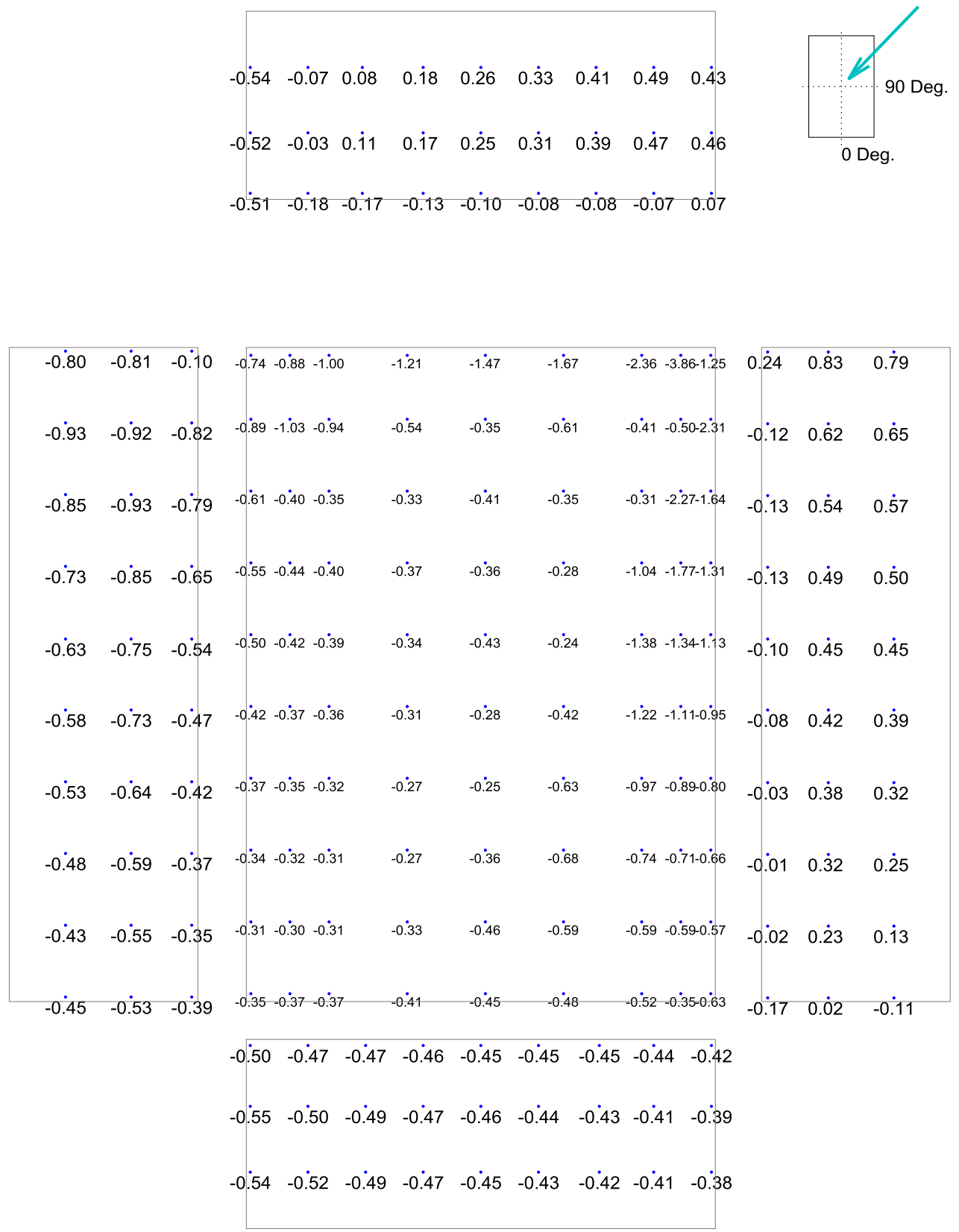

Figure $44-$ Mean $C_{p}$ at scale 1:6, tap-wise display 
$C p_{\text {mear }}$ Dir=135,SC 1:1
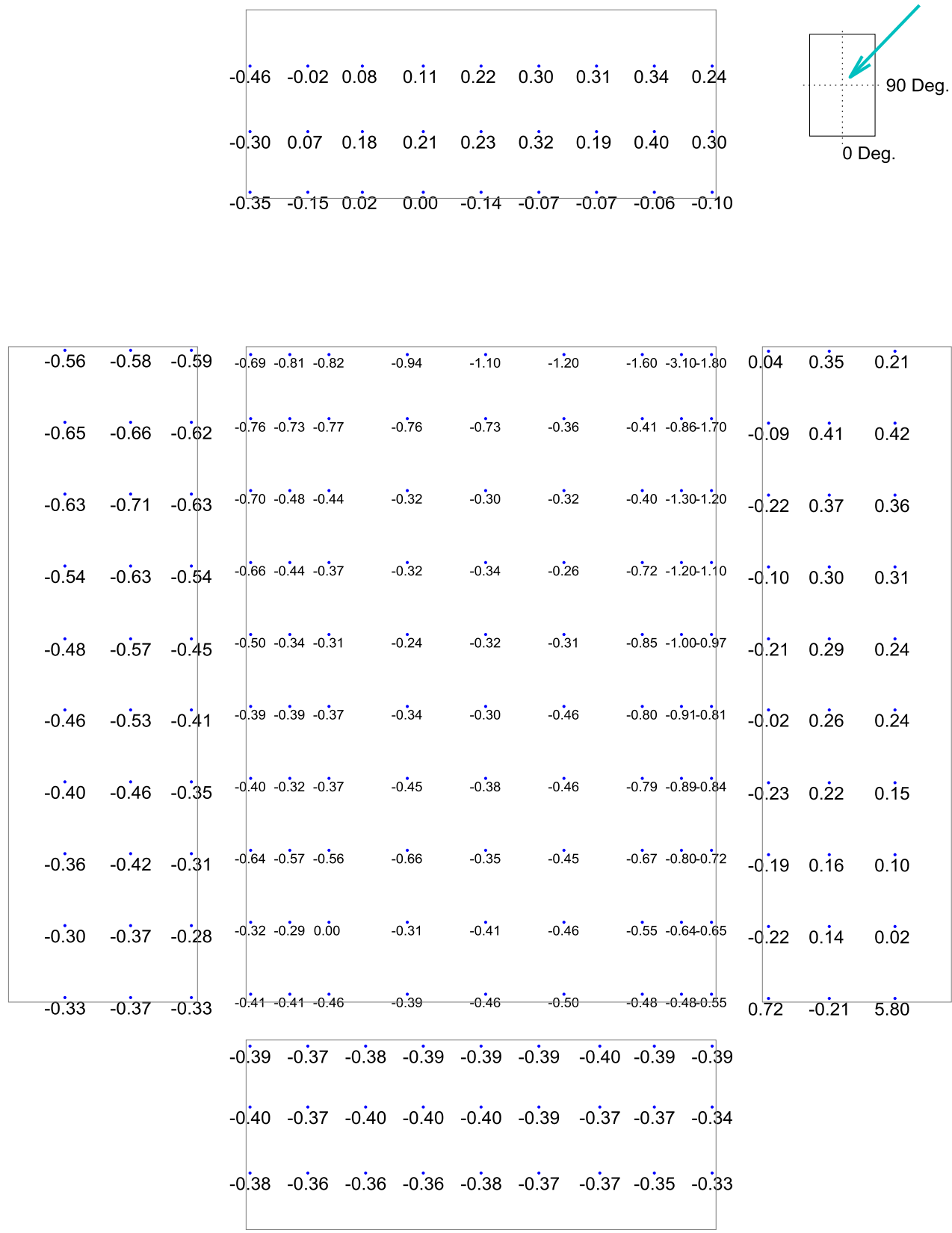

Figure $45-$ Mean $C_{p}$ at full-scale, tap-wise display 


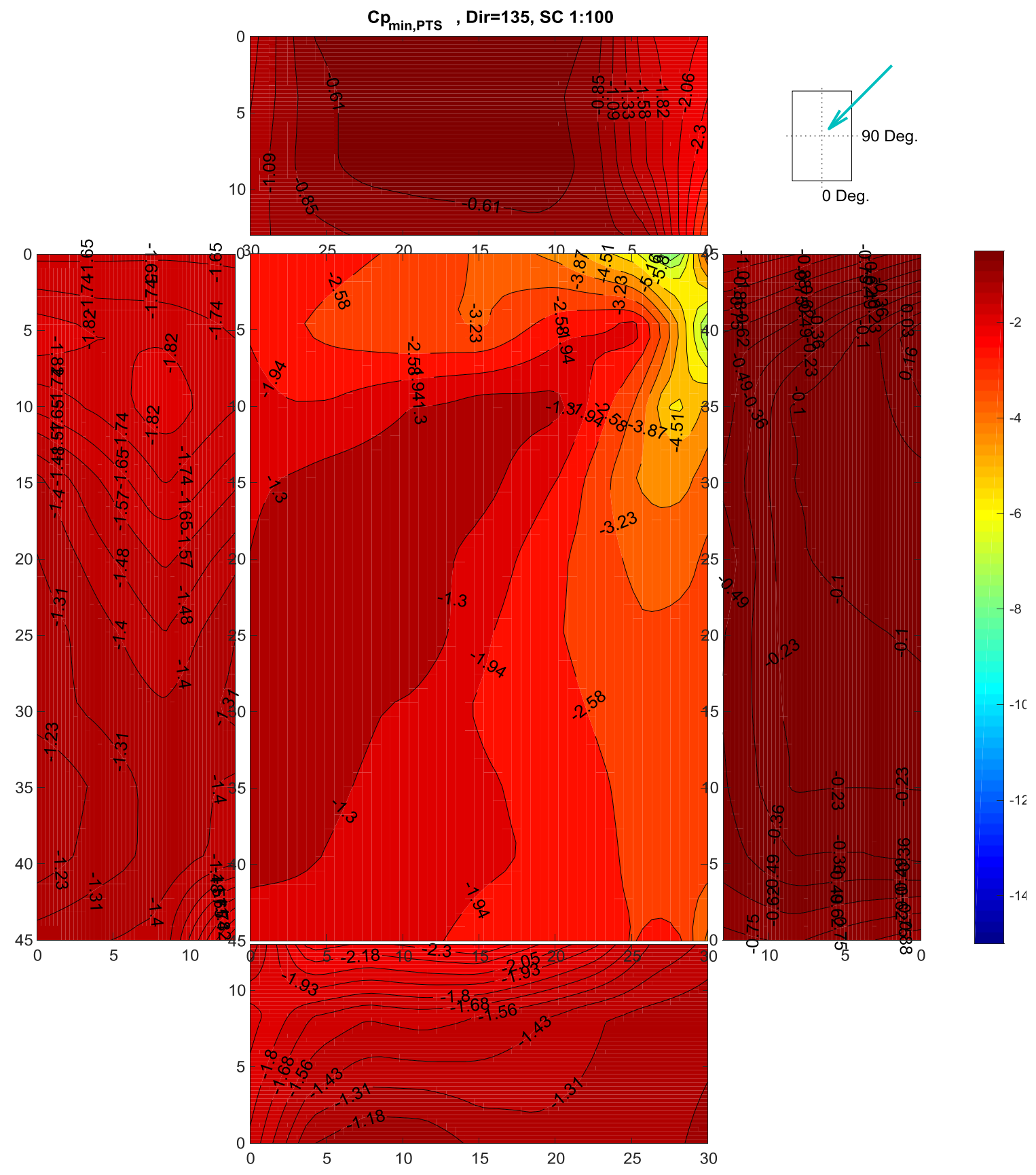

Figure $46-$ Peak $C_{p}$ distribution at 1:100 scale, Dir $=135^{\circ}$ 


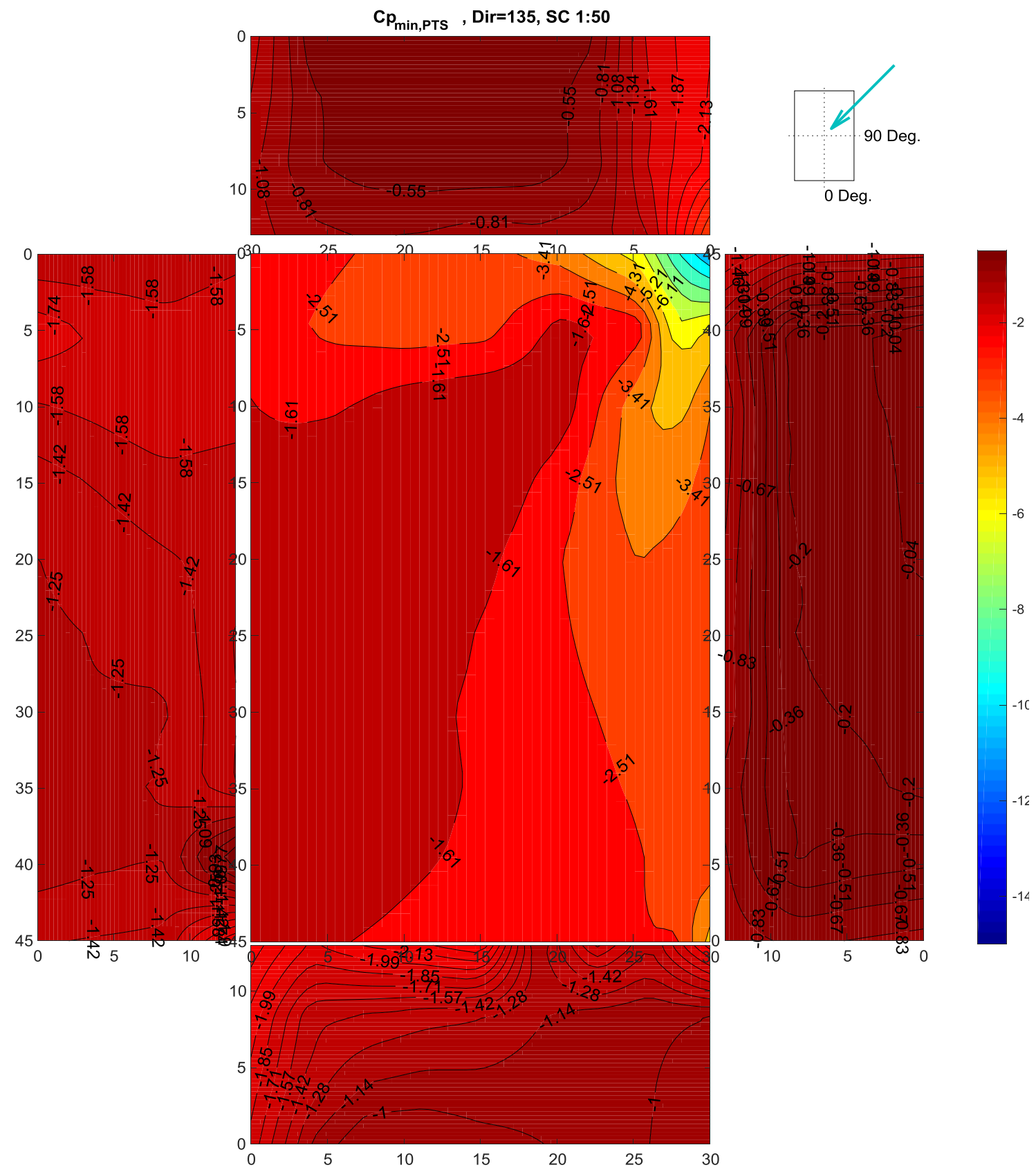

Figure $47-$ Peak $C_{p}$ distribution at 1:50 scale, Dir $=135^{\circ}$ 


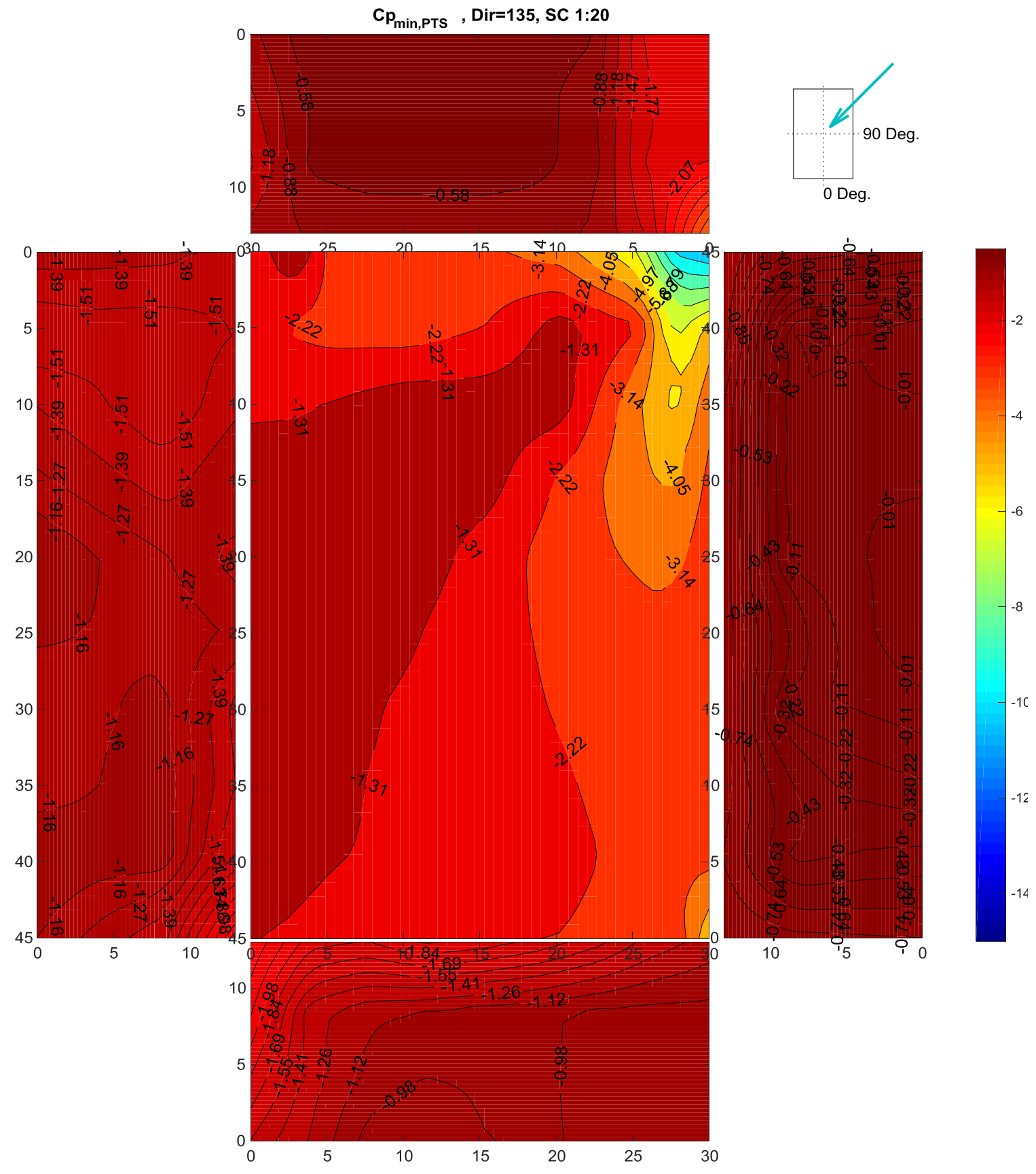

Figure $48-$ Peak $C_{p}$ distribution at 1:20 scale, Dir $=135^{\circ}$ 


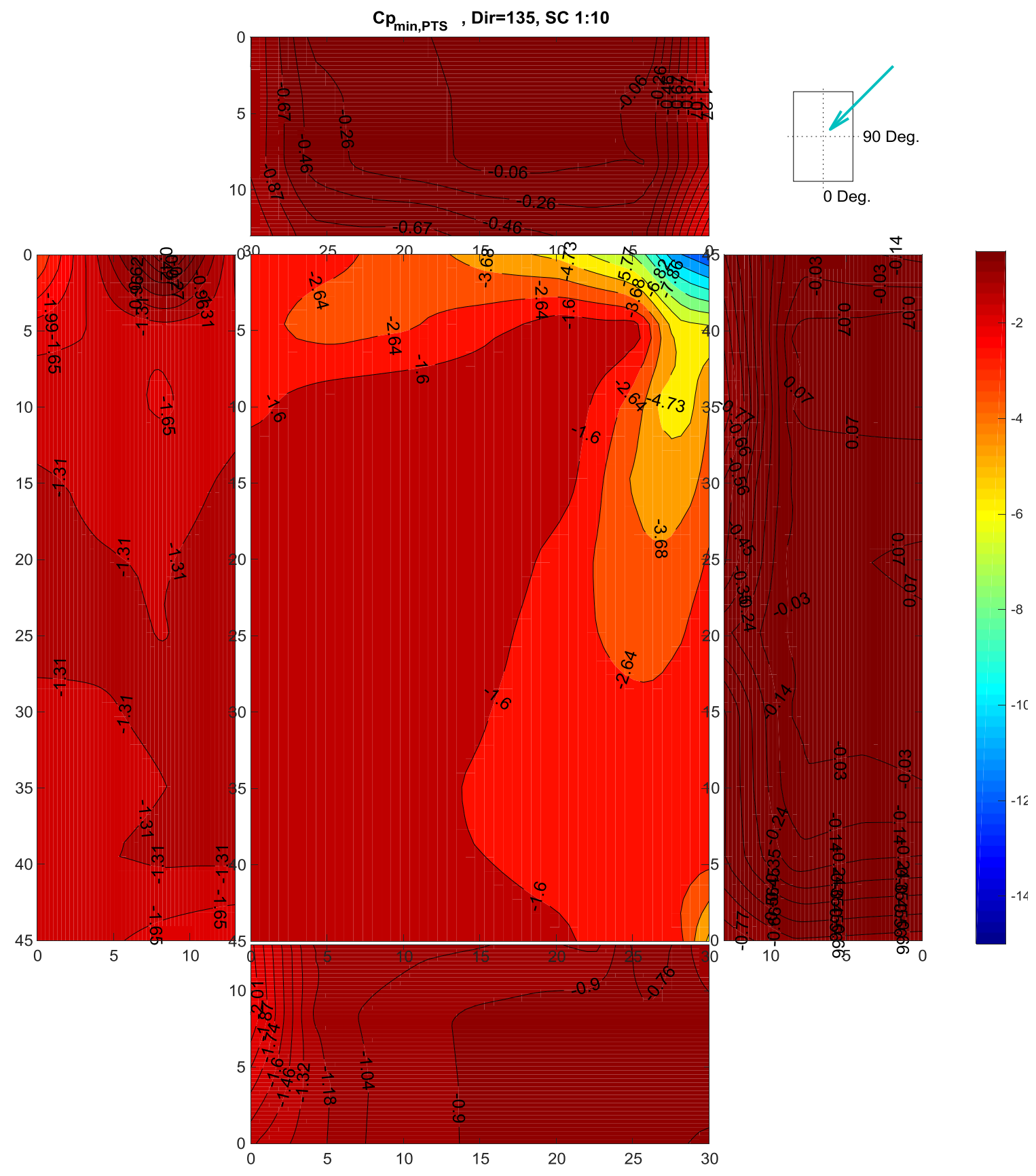

Figure $49-$ Peak $C_{p}$ distribution at 1:10 scale, Dir $=135^{\circ}$ 


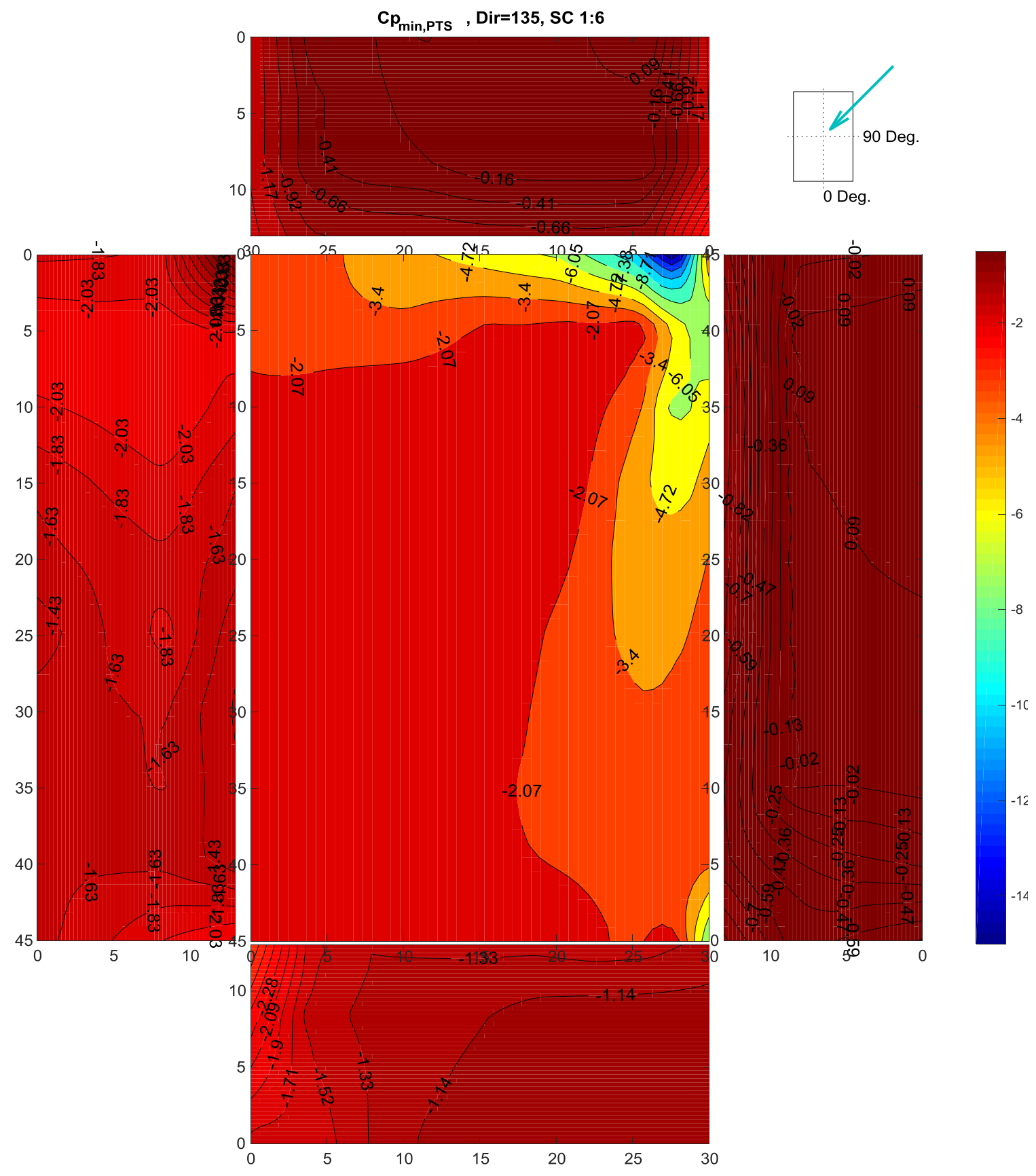

Figure $50-$ Peak $C_{p}$ distribution at 1:6 scale, Dir=135 


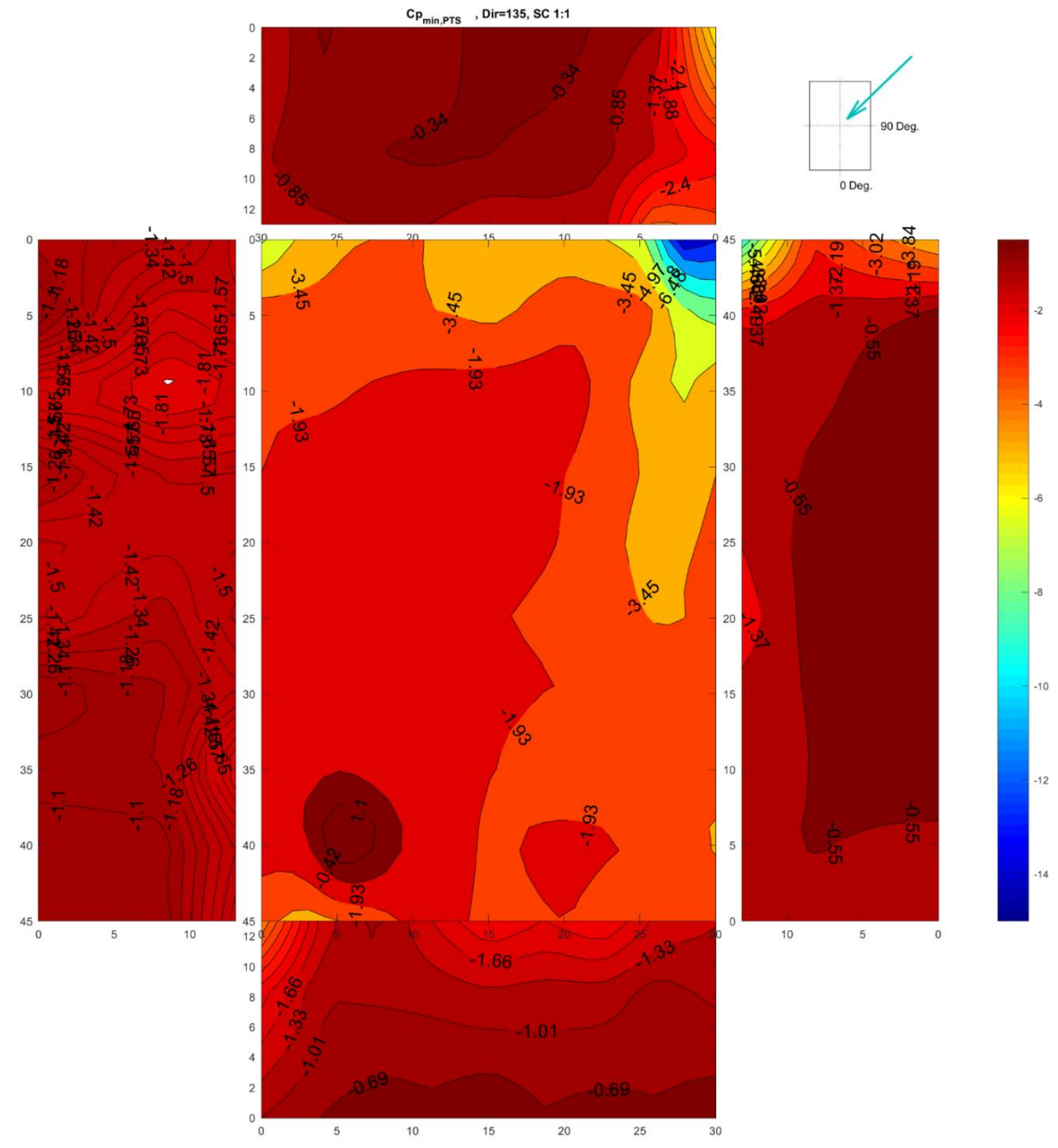

Figure $51-$ Peak $C_{p}$ distribution at full-scale, Dir $=135^{\circ}$ 


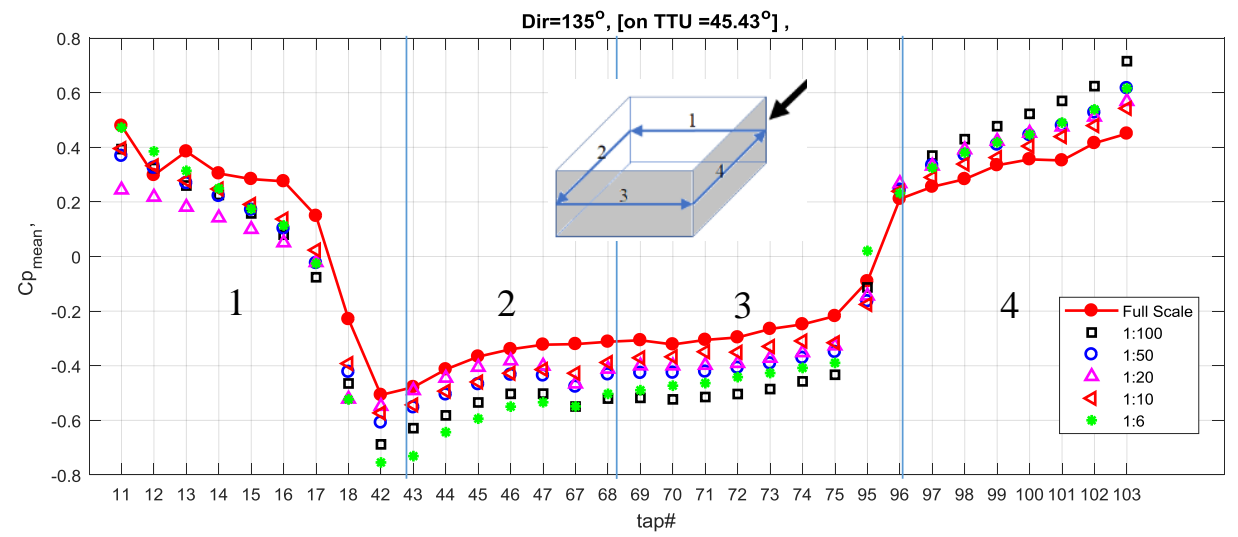

Figure $52-$ Mean $C_{p}$ on the walls - Dir $135^{\circ}$

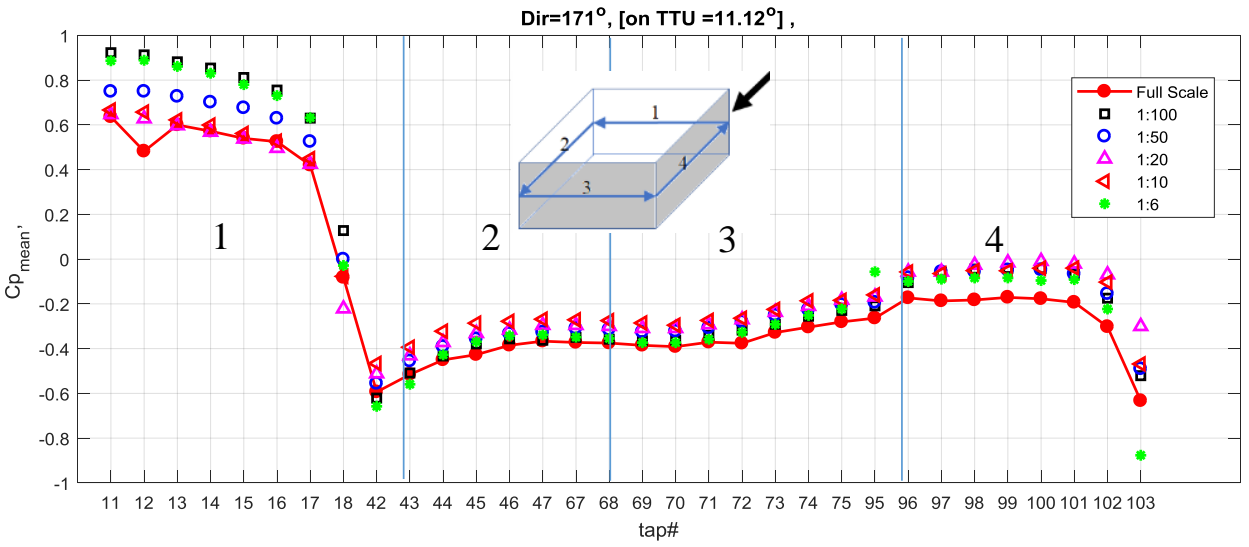

Figure $53-$ Mean $C_{p}$ on the walls - Dir $171^{\circ}$

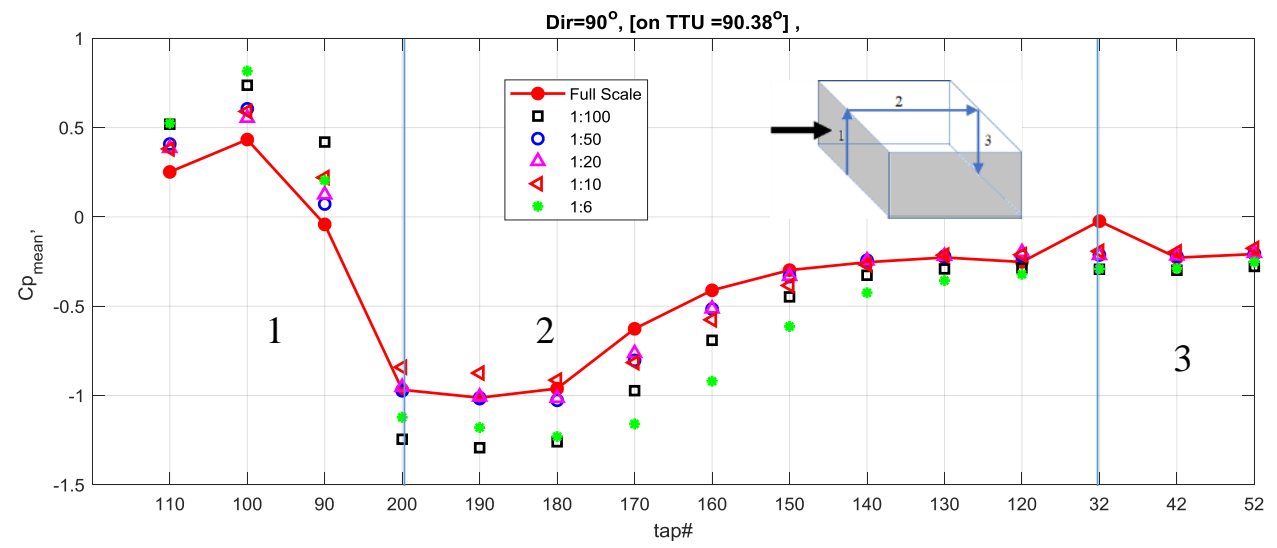

Figure $54-$ Mean $C_{p}$ on a Line of wall and roof taps at $90^{\circ}$ 

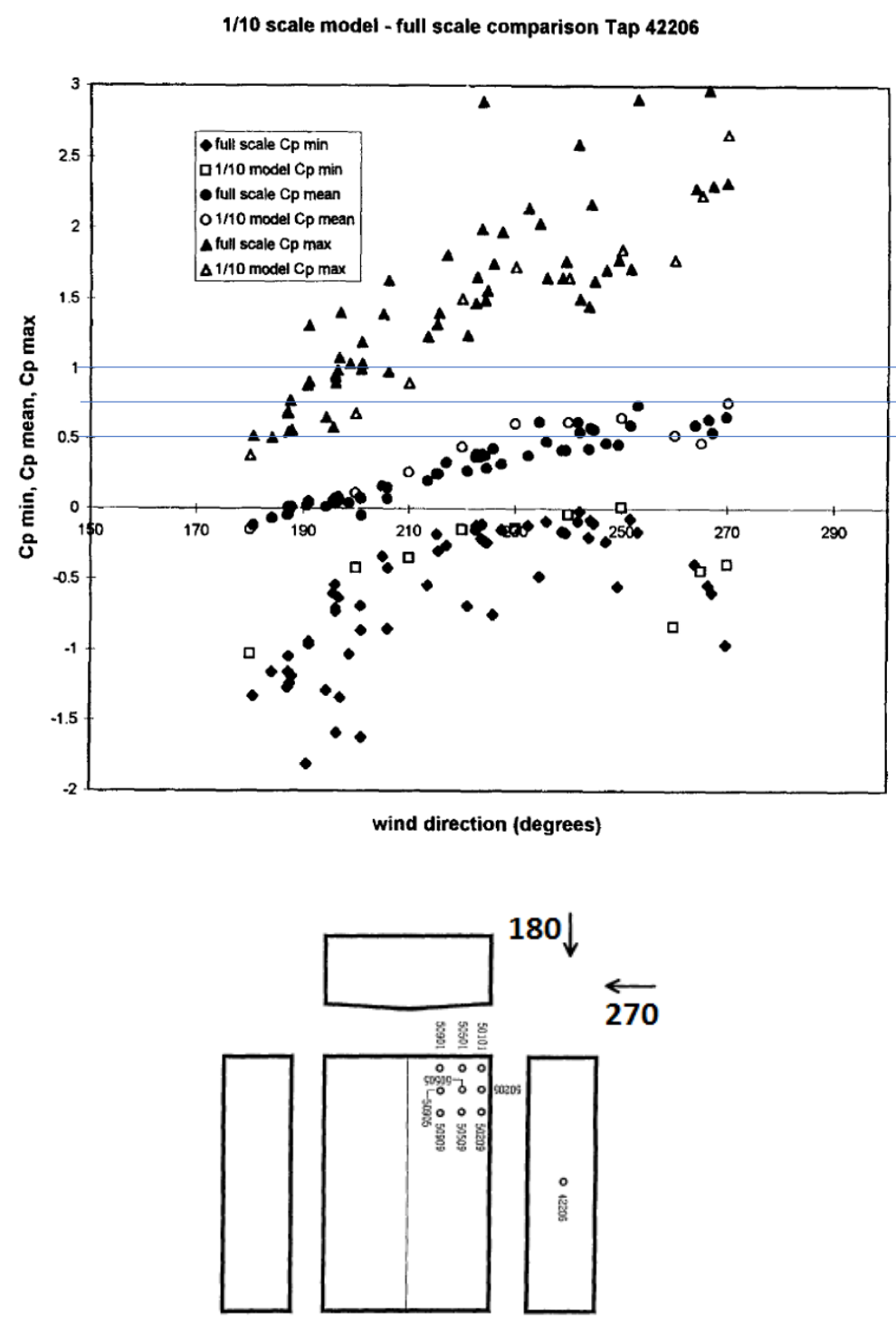

Figure 55 - Wall tap on TTU and a 1:10 model [13] 


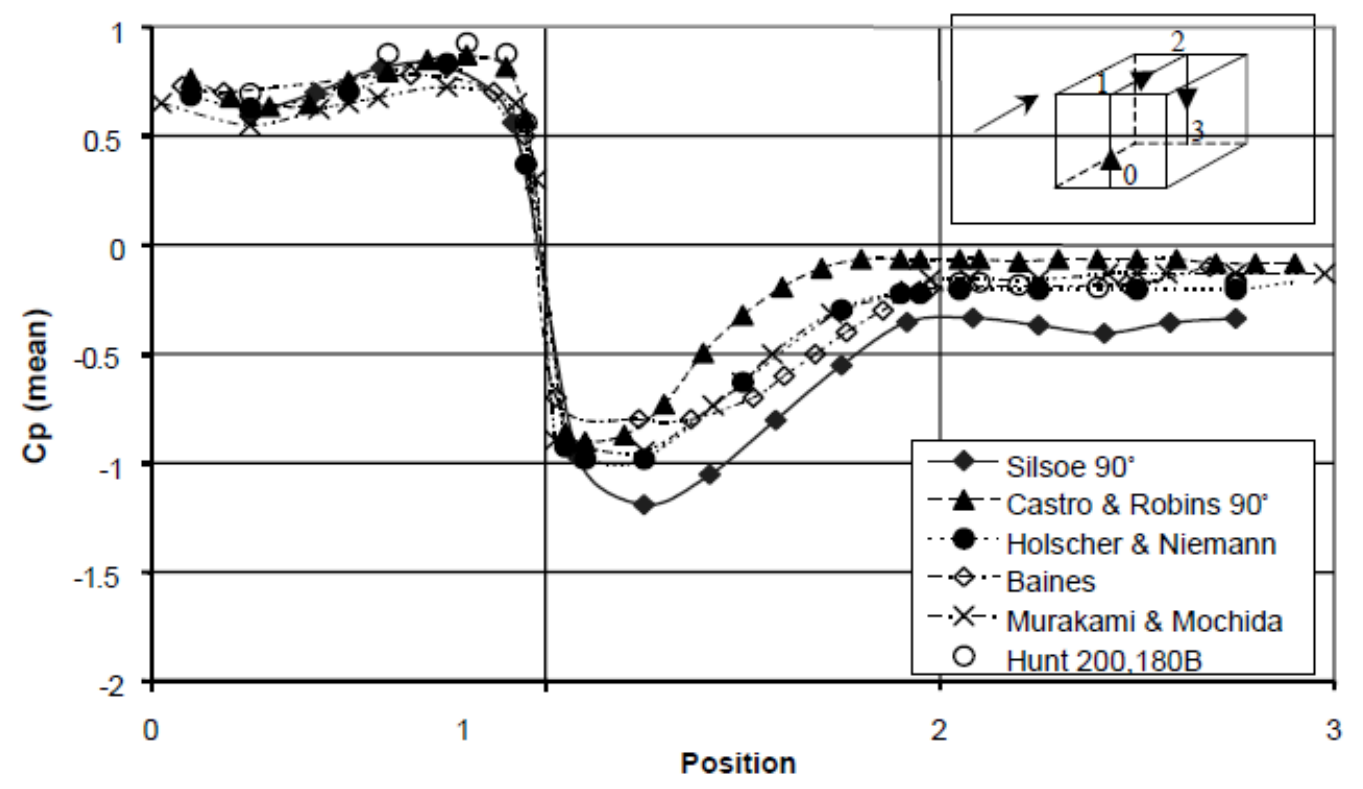

Figure 56 - Line of wall and roof taps at $90^{\circ}$ Silsoe Cube [21]

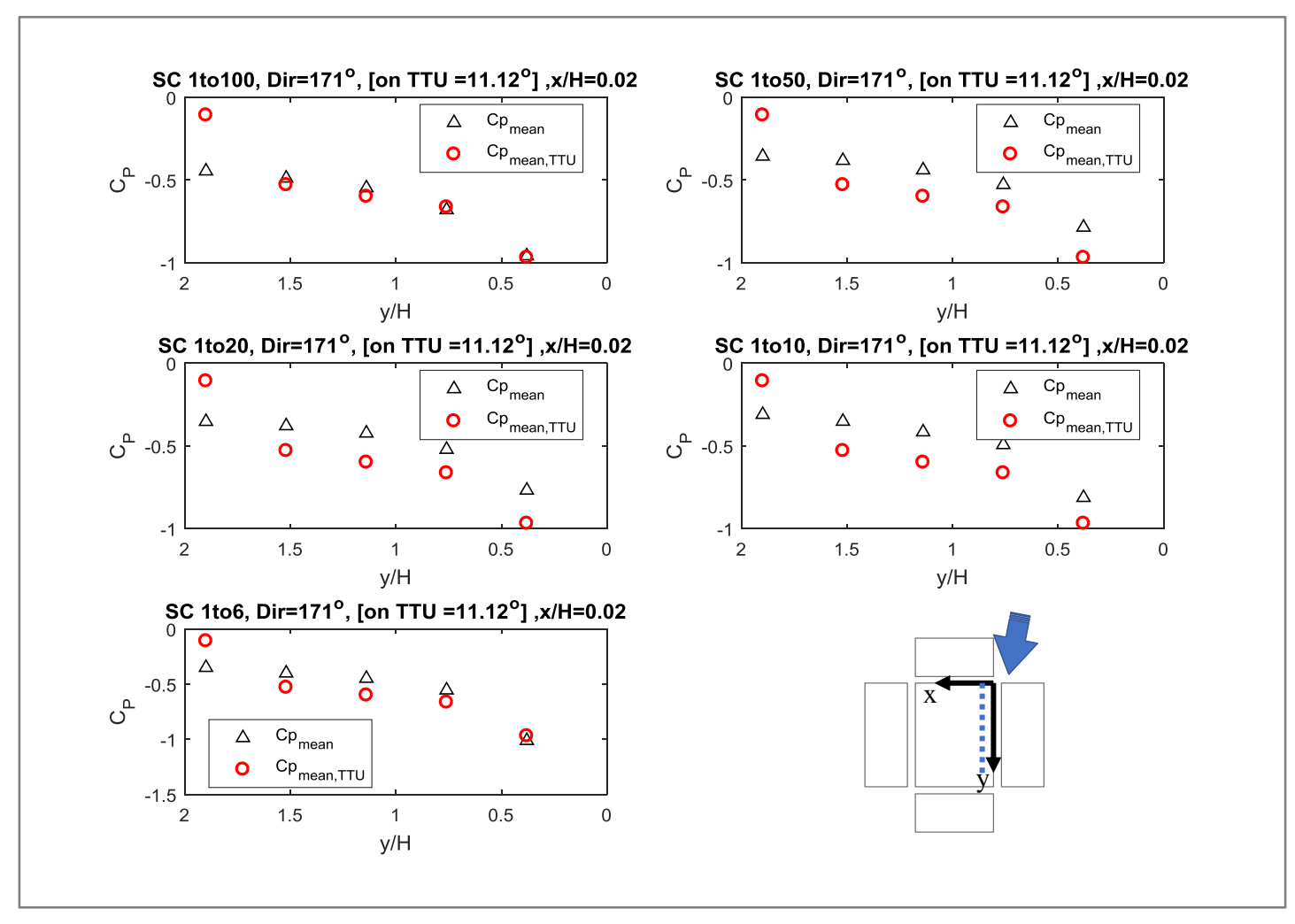

Figure 57 - Mean Cp of roof taps, Dir $171^{\circ}$ 

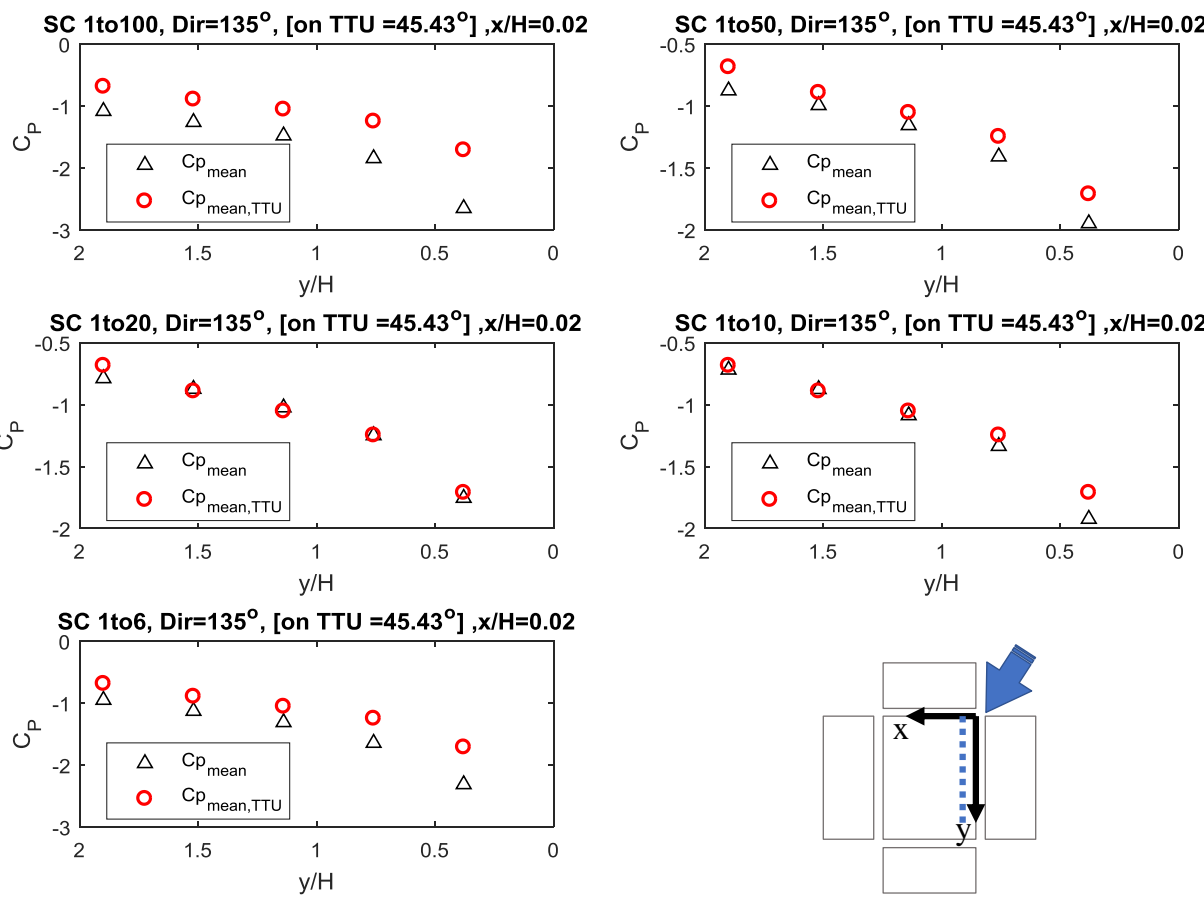

Figure 58 - Mean Cp of roof taps, Dir $135^{\circ}$

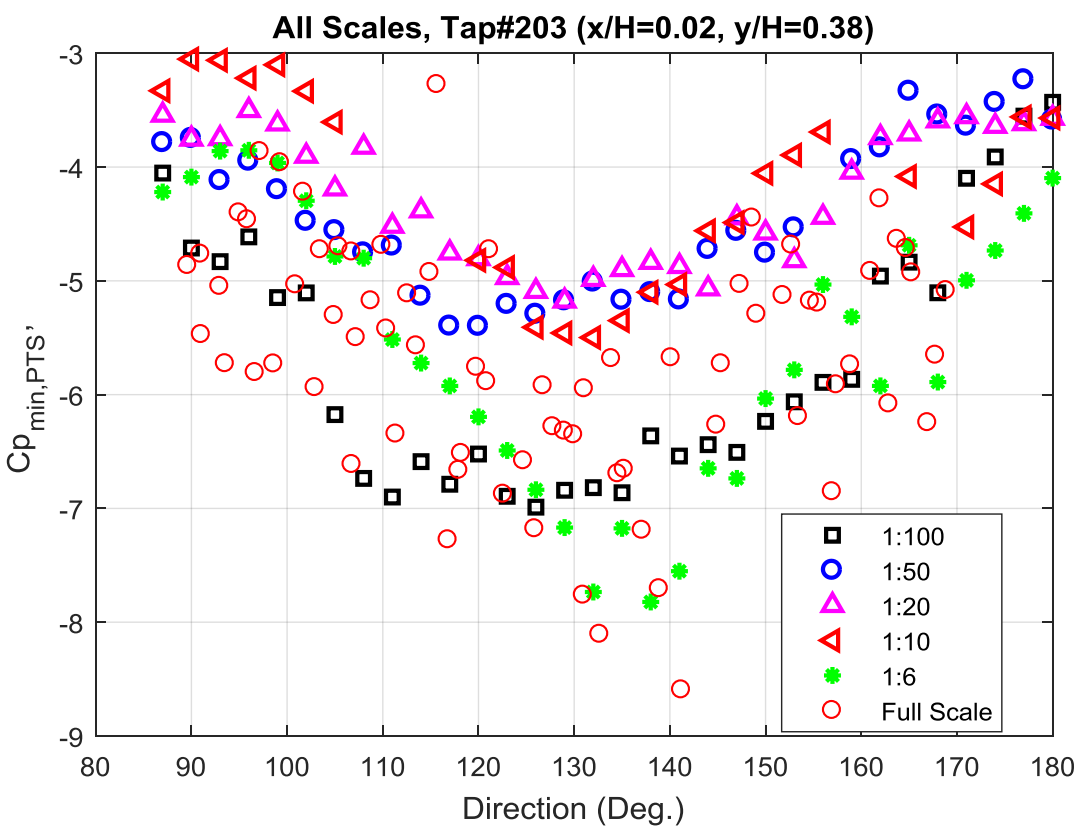

Figure 59 - Directional plot for tap\#203 


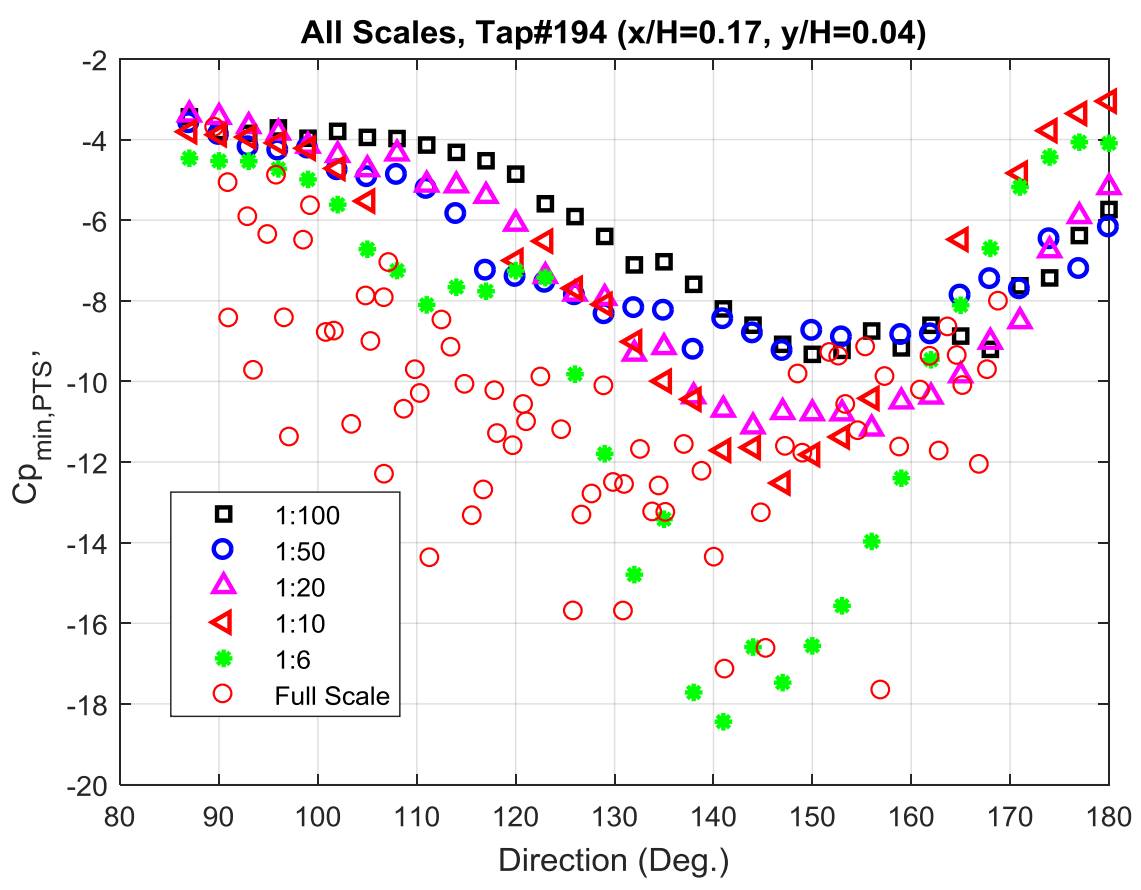

Figure 60 - Directional plot for tap\#194
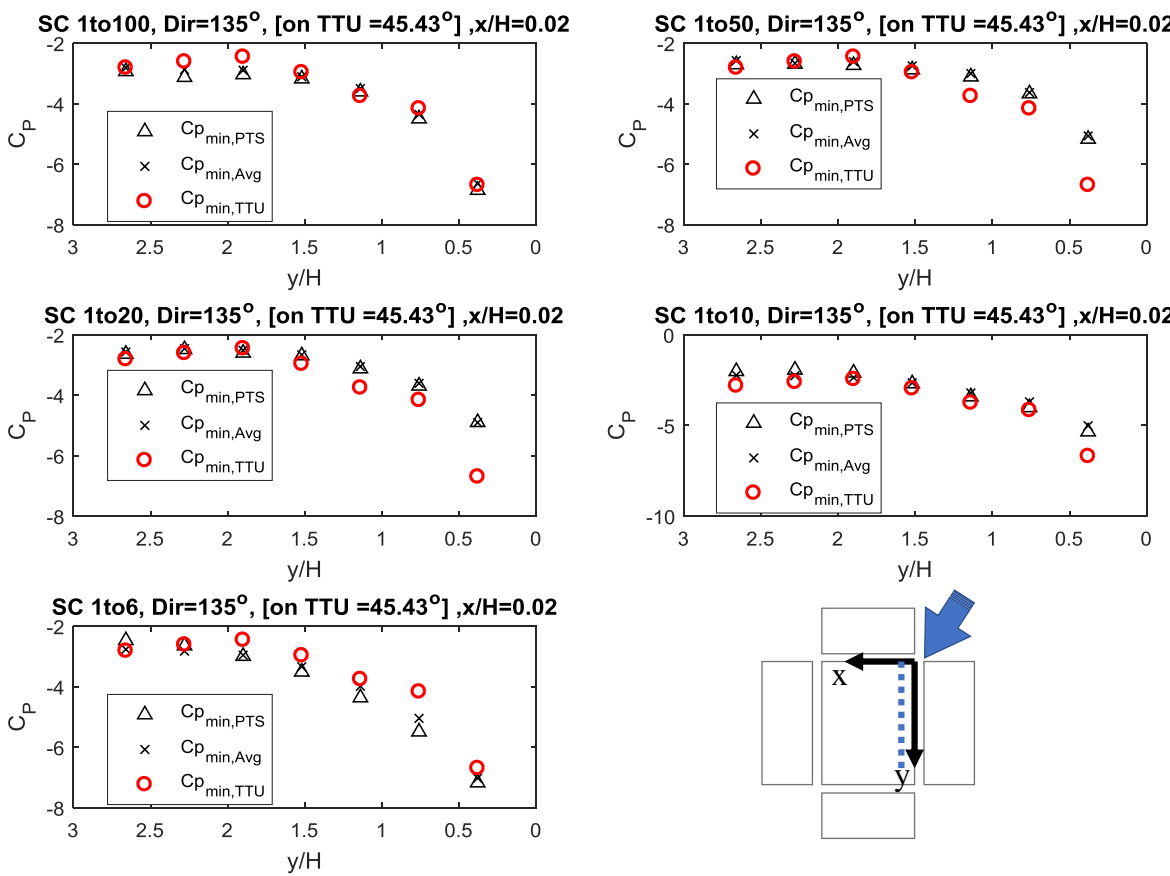

Figure $61-$ Peak roof suctions, Dir $=135, \mathrm{x} / \mathrm{H}=0.02$ 

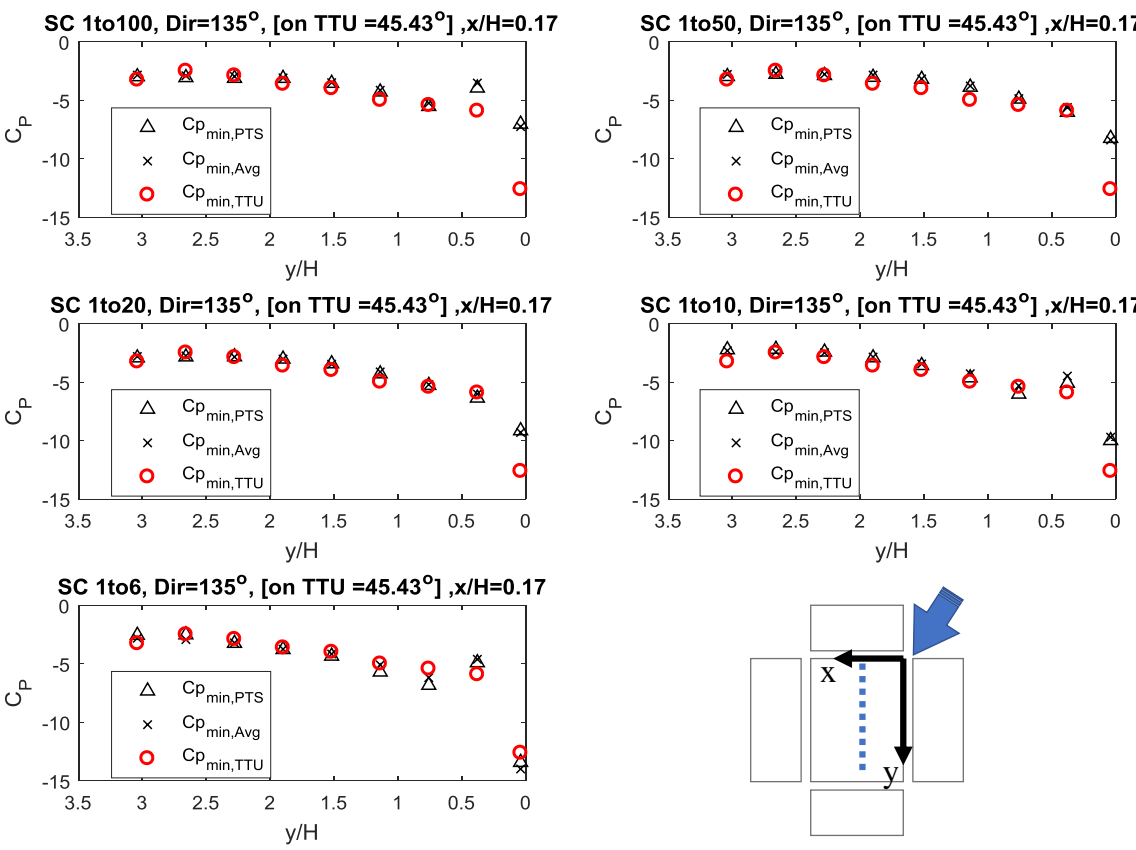

Figure $62-$ Peak roof suctions, Dir=135, x/H=0.17
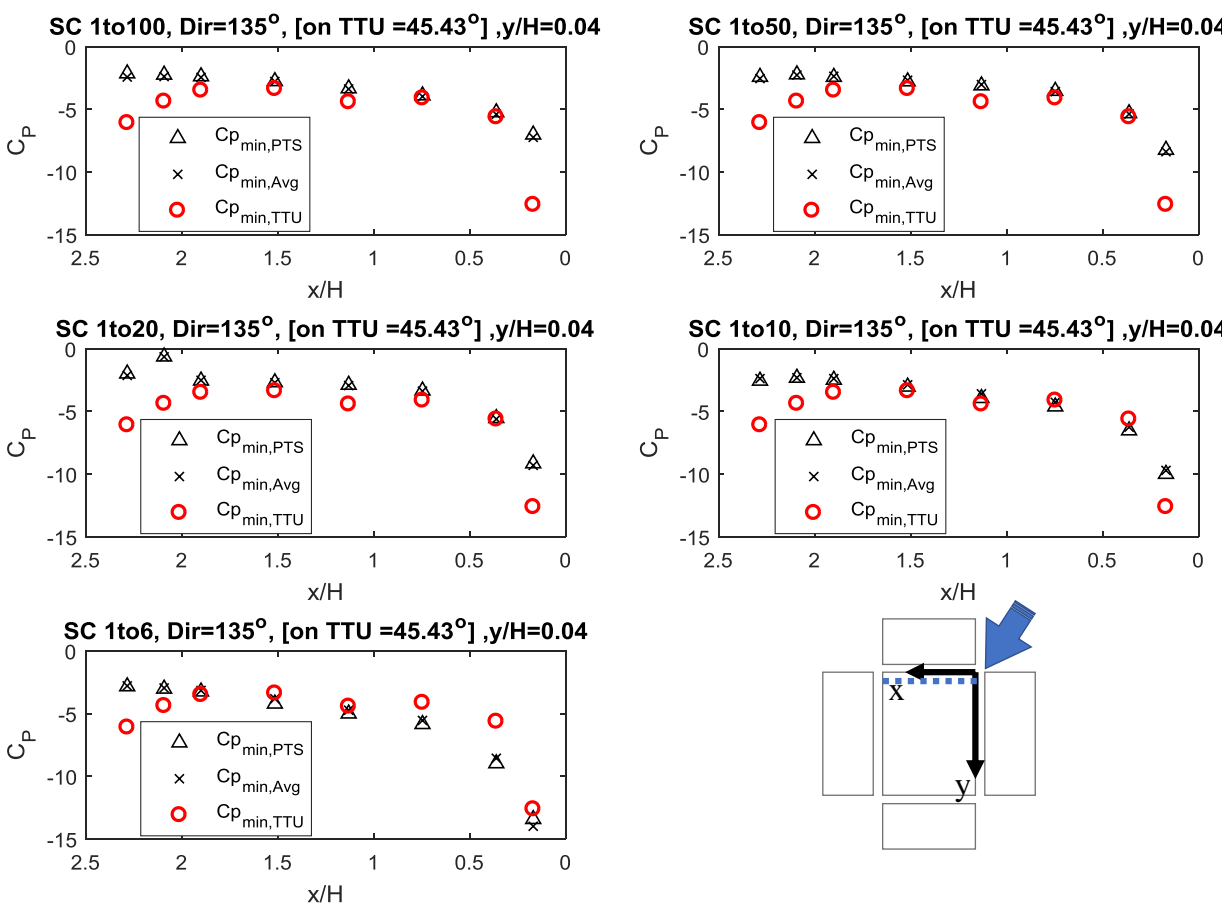

Figure $63-$ Peak roof suctions, Dir=135, y/H=0.04 

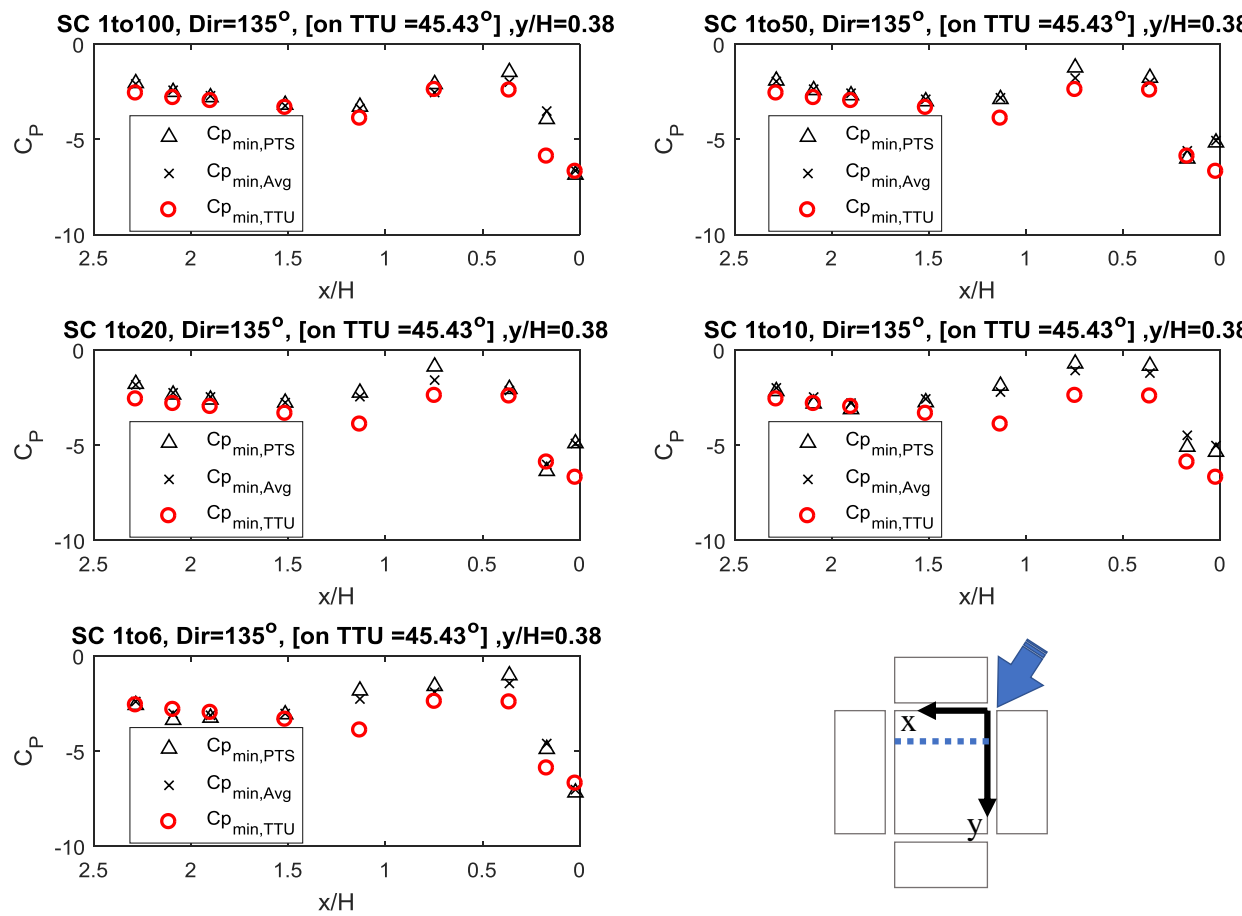

Figure $64-$ Peak roof suctions, Dir=135, y/H=0.38
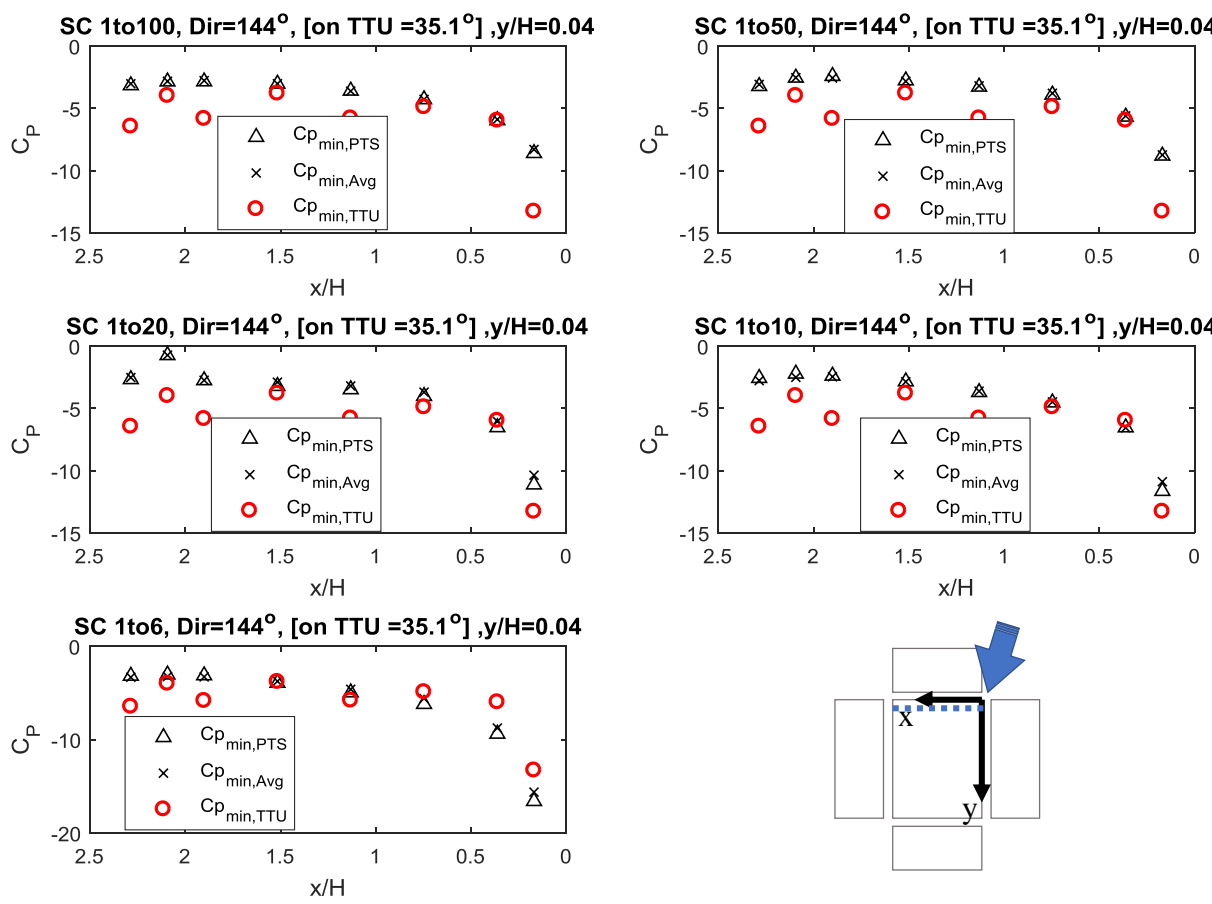

Figure $65-$ Peak roof suctions, Dir=144, y/H=0.04 

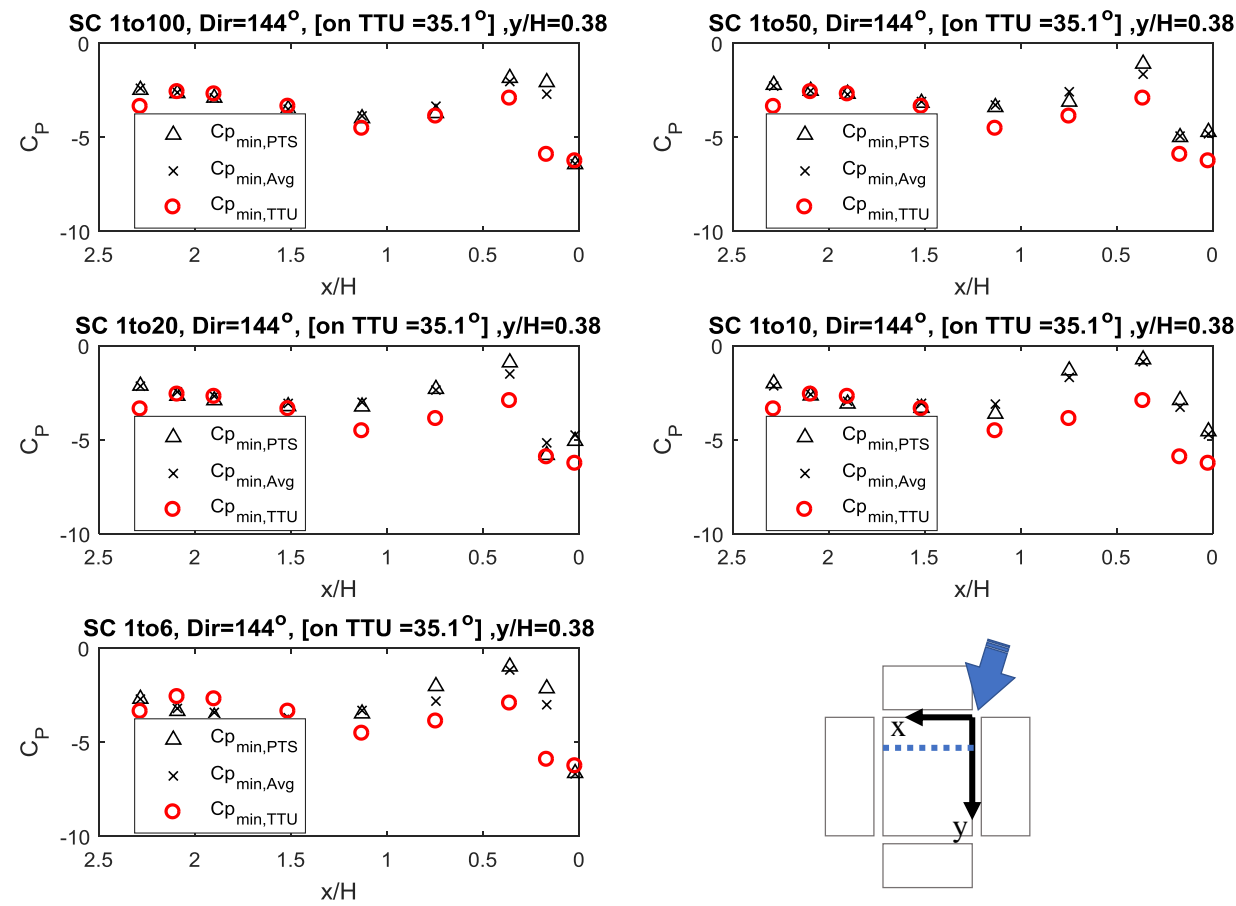

Figure $66-$ Peak roof suctions, Dir $=144, y / H=0.38$
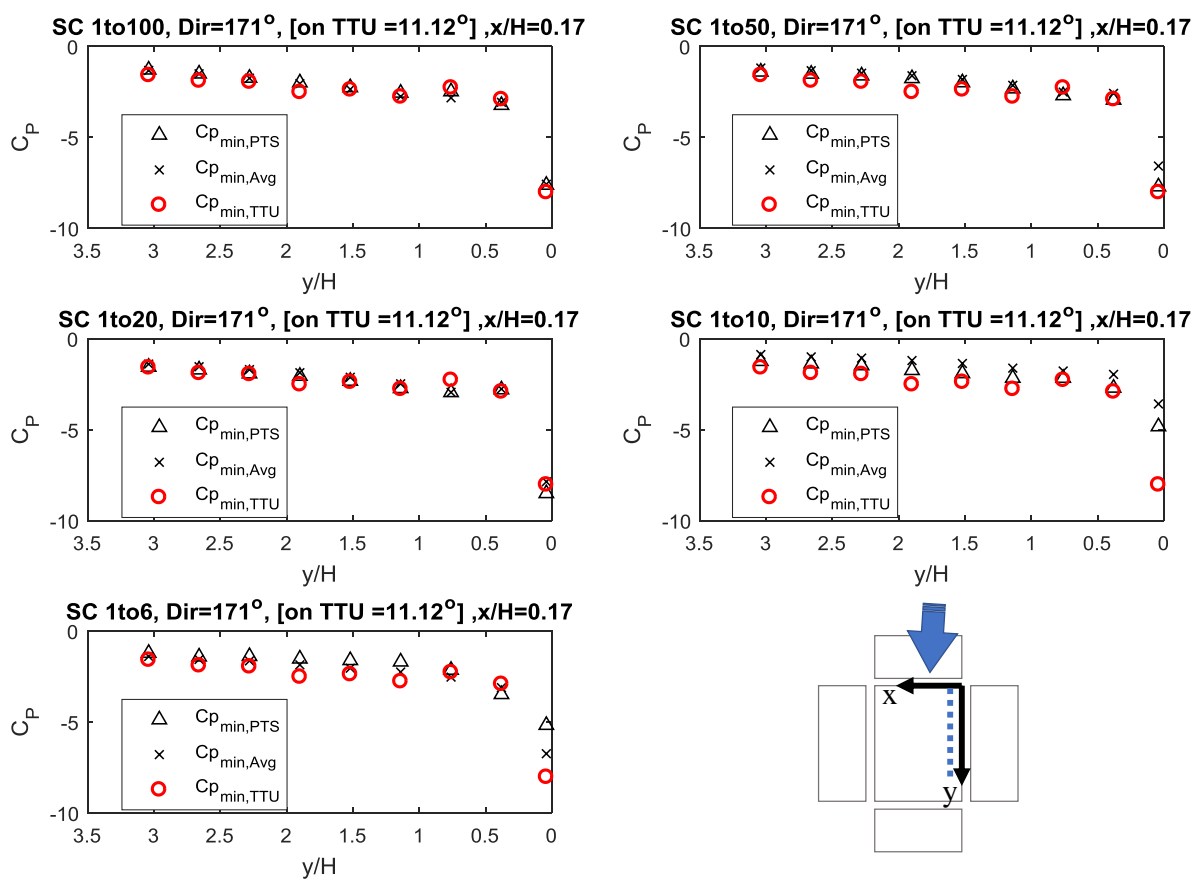

Figure $67-$ Peak roof suctions, Dir=171, x/H=0.17 


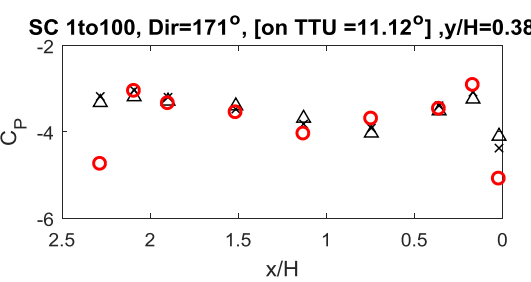

SC 1 to20, Dir $=171^{\circ}$, [on TTU $=11.12^{\circ}$ ] ,y/H=0.38

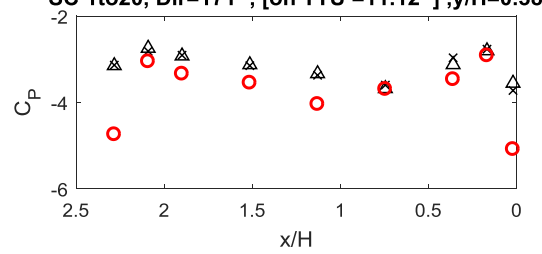

SC 1to6, $\operatorname{Dir}=171^{\circ}$, [on TTU $=11.12^{\circ}$ ],$y / H=0.38$

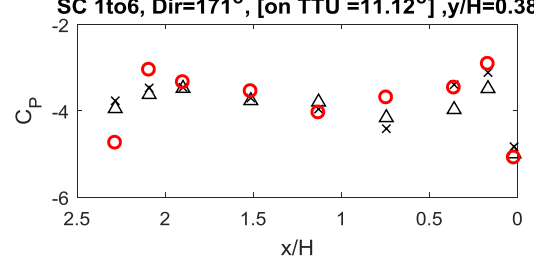

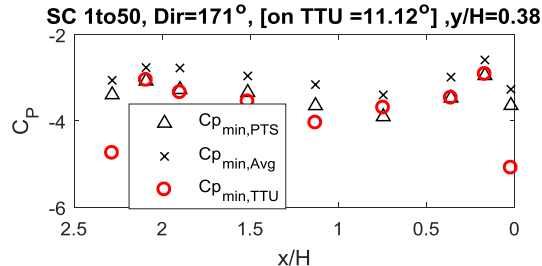

SC 1 to 10, Dir $=171^{\circ}$, [on TTU $=11.12^{\circ}$ ],$y / H=0.38$
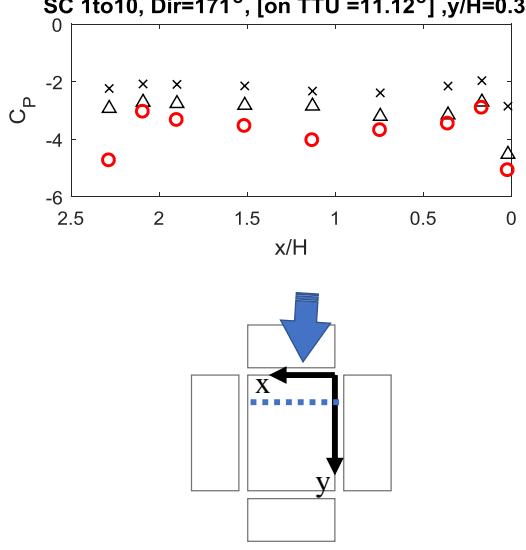

Figure $68-$ Peak roof suctions, Dir=171, y/H=0.38
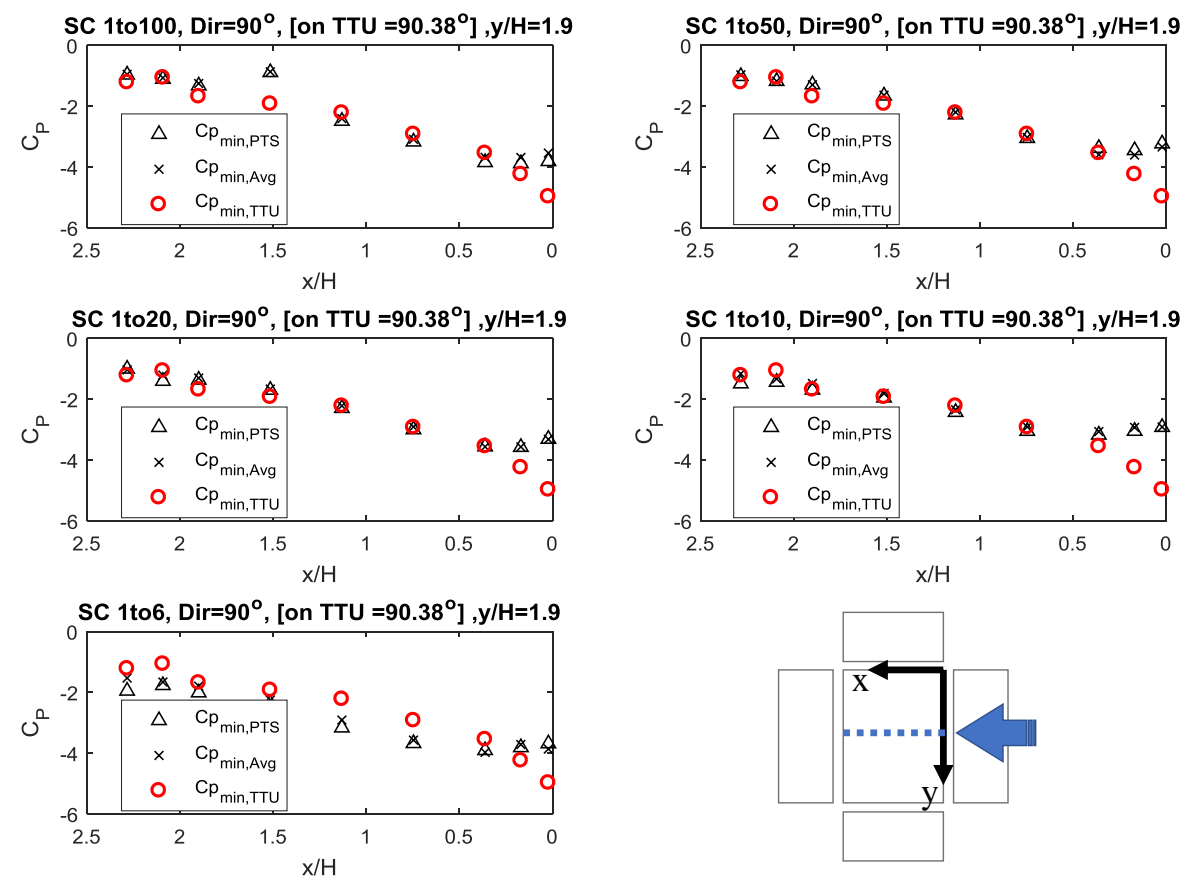

Figure $69-$ Peak roof suctions, Dir=90, y/H=1.9 


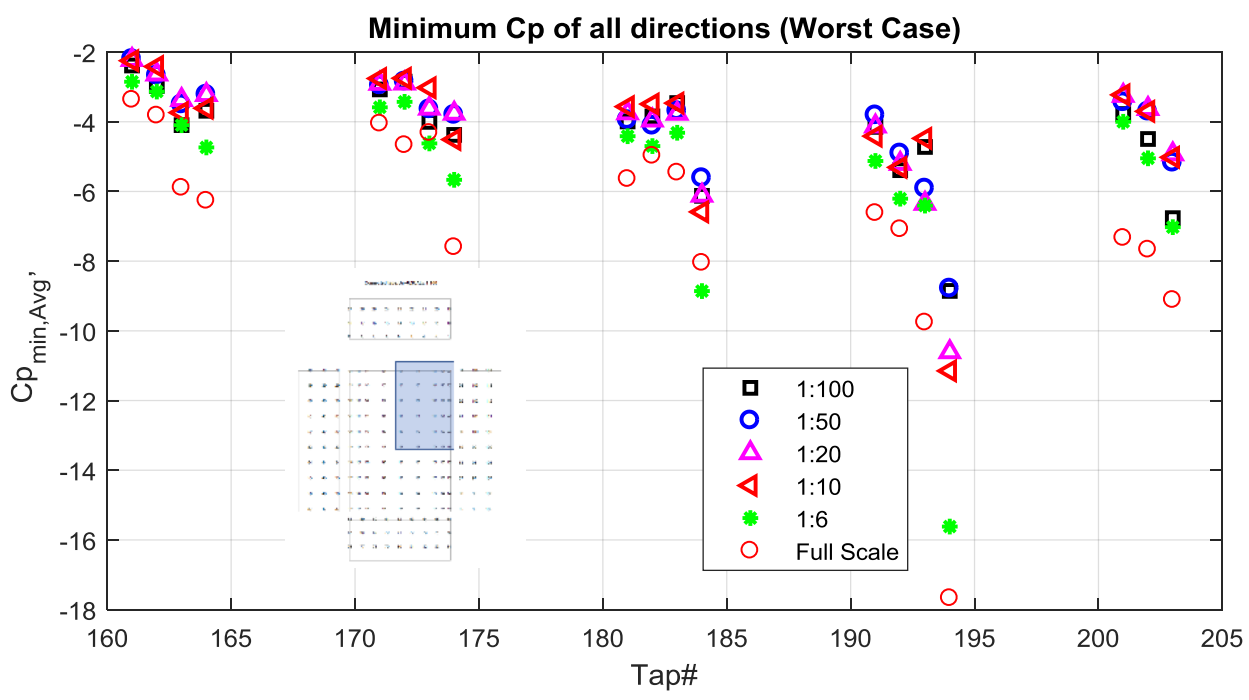

Figure 70 - Minimum $C_{p \text { min,Avg }}$ of all directions (worst case scenario)

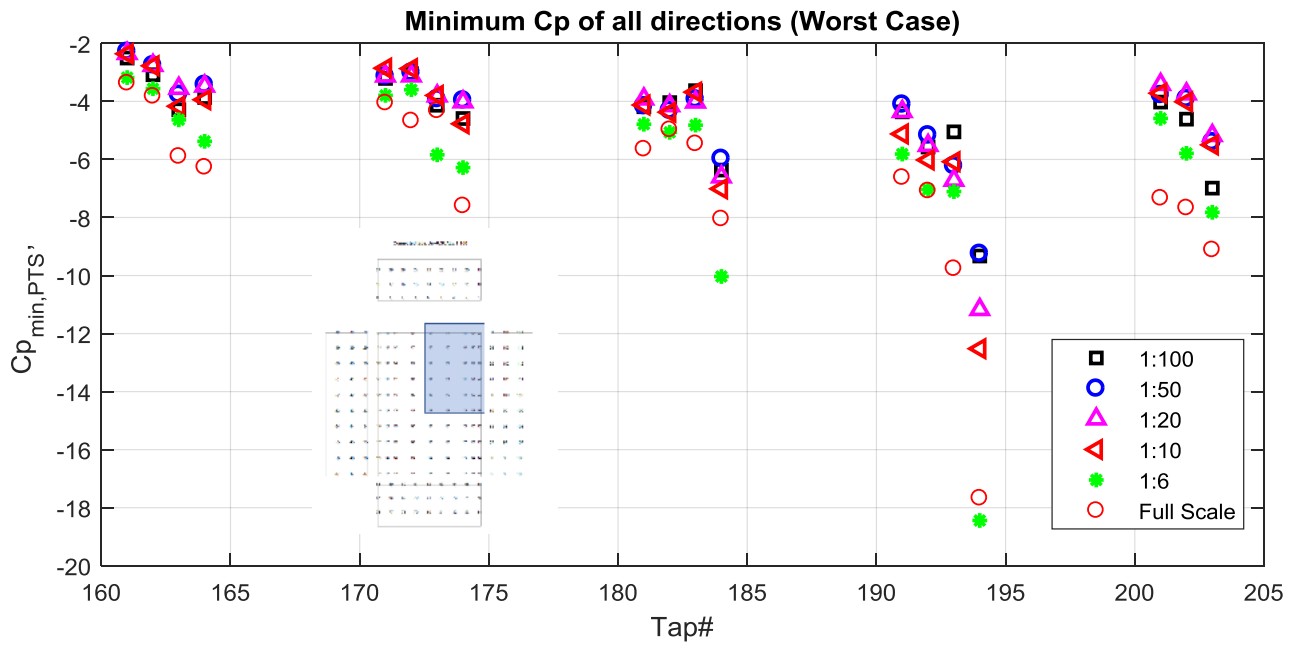

Figure 71 - Minimum $\mathrm{C}_{\mathrm{p}, \min }$ of all directions (worst case scenario) 


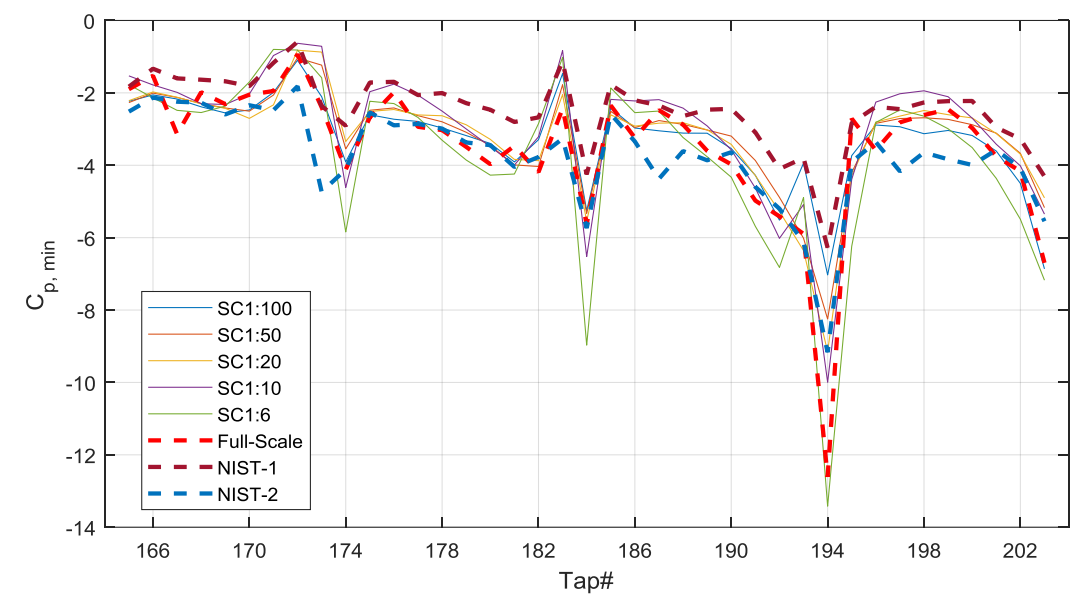

Figure 72 - Comparison between WOW results, TTU (full-scale) \& NIST

\subsection{Evaluation of Scaling and Reynolds Number Effects}

As the model size increases, it gets increasingly difficult to maintain scaling for the relevant integral length scale of atmospheric turbulence to be same as the scale used to model the building (see ASCE 7-16). For larger models relatively less large-scale turbulence in the upcoming flow can be generated. Since the large-scale turbulence (large eddies) fluctuate at lower frequencies, the resulting turbulence power spectra show a lack of low-frequency turbulence content. This shortage progressively increases as the model scale is increased and as discussed earlier, the trend can be apparently seen in the turbulence power spectra generated for different model scales (Figure 29). Based on that one should expect the extent of PTS correction on the observed peaks also to progressively become larger as the model scale increases. This concept is apparently comprehensible from the comparison of observed peaks vs. PTS peaks at various scales. Figure 73 shows the results for the roof tap 194 at all the five tested model scales. At the lower scales the plots of the observed peaks and those after applying the PTS are almost the same. But at the larger models, i.e., 1:10 and 1:60, the application of PTS generates peak pressures that are 
significantly larger (in magnitude) as compared to the observed values. The 1:100 model observed peaks are even slightly higher than the PTS-based peaks which could be the result of excessive turbulence content generated at the model height, as it can be seen in the turbulence power spectra as well. A similar trend is observed for the tap 203 as shown in Figure 74. Also as an example of the peak positive pressure coefficients, the results from wall tap 100 are shown in Figure 75 where the observed trend conforms with the results from other two taps reported and shows as the model scales are larger (e.g., 1:10 and 1:6), there occurs a significant peak correction by the PTS method. So, if an extreme value (EV) method is adopted to estimate the peaks at the small scales, one may expect to see a good agreement between those peaks and the full-scale results from field data; however, Reynolds number and scaling effects may affect such results. To assess the performance of the EV method, a Gumbel fit was used where the fit parameters were estimated by the Lieblein's best linear unbiased estimation (BLUE) method [63]. The comparison between the peaks estimated by the BLUE method and PTS peaks is presented in Figure 76 and Figure 77. The BLUE method can only predict the peak based on the statistical procedure inherent in the EV approach; however, unlike PTS, the BLUE method is unable to compensate for the missing turbulence as the model size increases. This is apparent from Figures 62 and 63 where it is shown that for 1:10 and 1:6 models the PTS-based peaks cam match those from the field data more closely as compared to those obtained using the BLUE method of peak estimates. The mismatch of peaks for smaller models (1:100, 1:50, 1:20), whether using BLUE or PTS, can be attributed to the violation of Reynolds number simulation and will be investigated in more details here. 
To study the Reynolds number effect, some of the tests were run at higher wind speeds (Table 3) and the pressure data was collected at few numbers of taps. Figure 78 shows the peak pressure coefficients from the 1:50 model that are compared with full-scale TTU data for a few number of roof taps. According to this figure, the increase of the Reynolds number has led to a slight improvement in the test results; however, it should be considered that the Re difference is just one order of magnitude due to the small dimensions of the 1:50 model.

To have a wider range of Re variations, the results from the smallest model (i.e. 1:100 scale) are compared to those from the largest model (i.e. 1:6 scale). Although the model dimensions are different, the blockage ratio is very minimal [64] (5\% for the 1:6 model) and still the Reynolds number should be the key factor contributing to the difference in the results between these two models. Figure 79 shows the $C_{p \text { min }}$ results from the two selected models and the full-scale TTU building at several roof taps. The Reynolds number at the 1:100 model was calculated to be $3.65 \times 10^{4}$ and was $2.4 \times 10^{6}$ for the $1: 6$ model, which when compared to the $\mathrm{Re}=2.05 \times 10^{6}$ at the full-scale shows the same order of magnitude has been achieved at the largest tested model. As displayed in Figure 79, the highest peak which has occurred at the roof corner tap 194, was very well predicted by the application of PTS to the pressure data from the 1:6 model. At the 1:100 model though the lower magnitude peaks were reasonably estimated by the PTS approach, but it was not possible to reproduce the highest peaks observed at full-scale. 

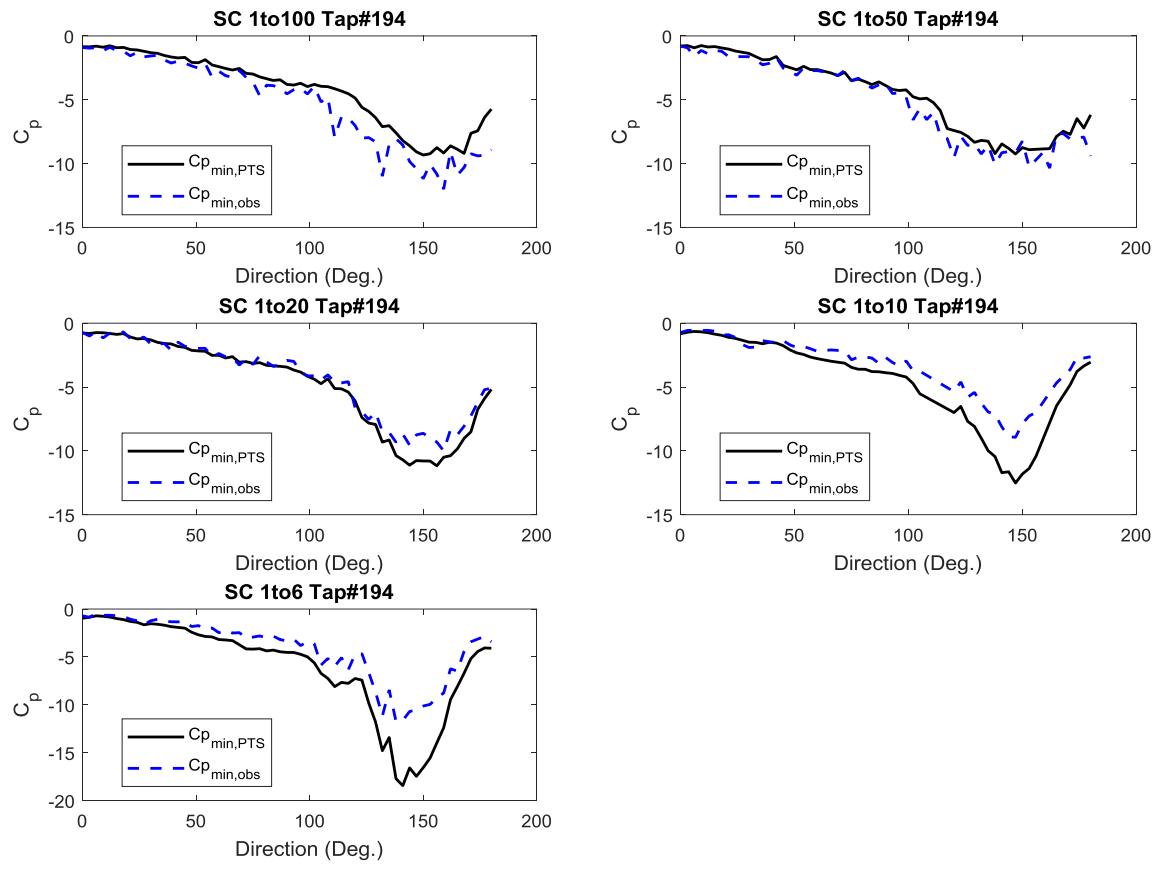

Figure 73 - Observed peaks vs. PTS peaks, roof tap\#194
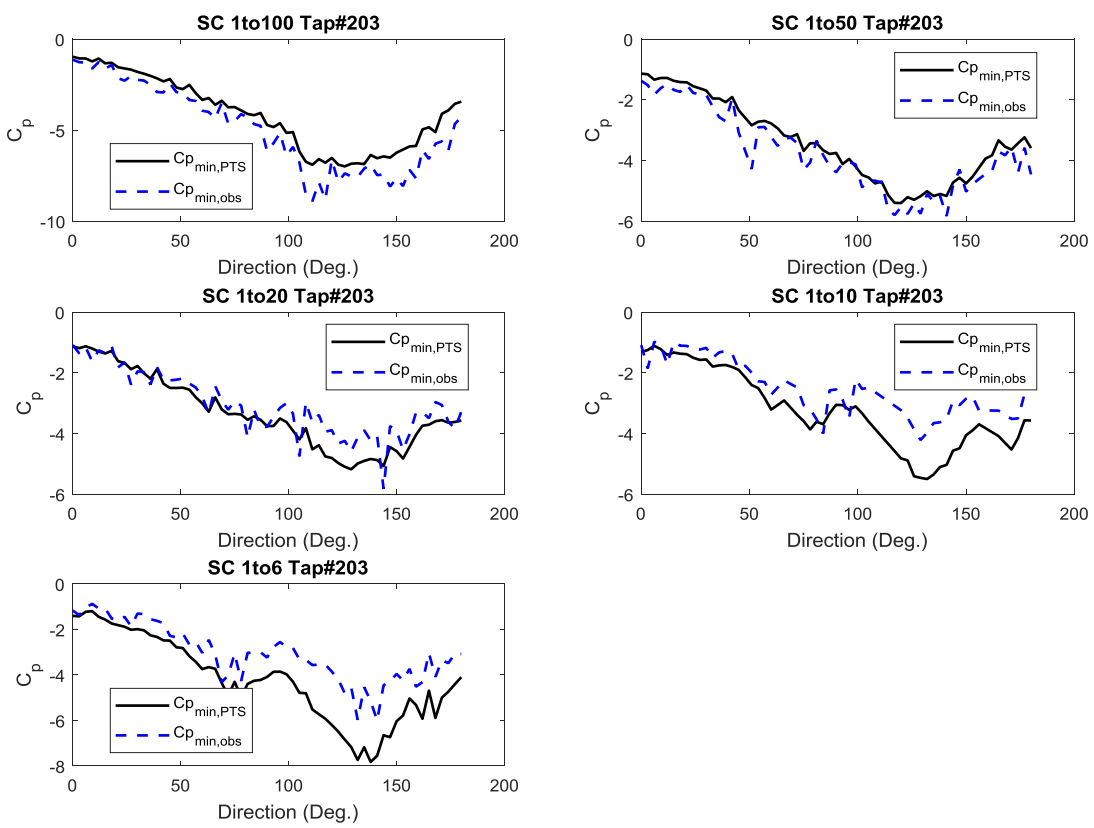

Figure 74 - Observed peaks vs. PTS peaks, roof tap\#203 

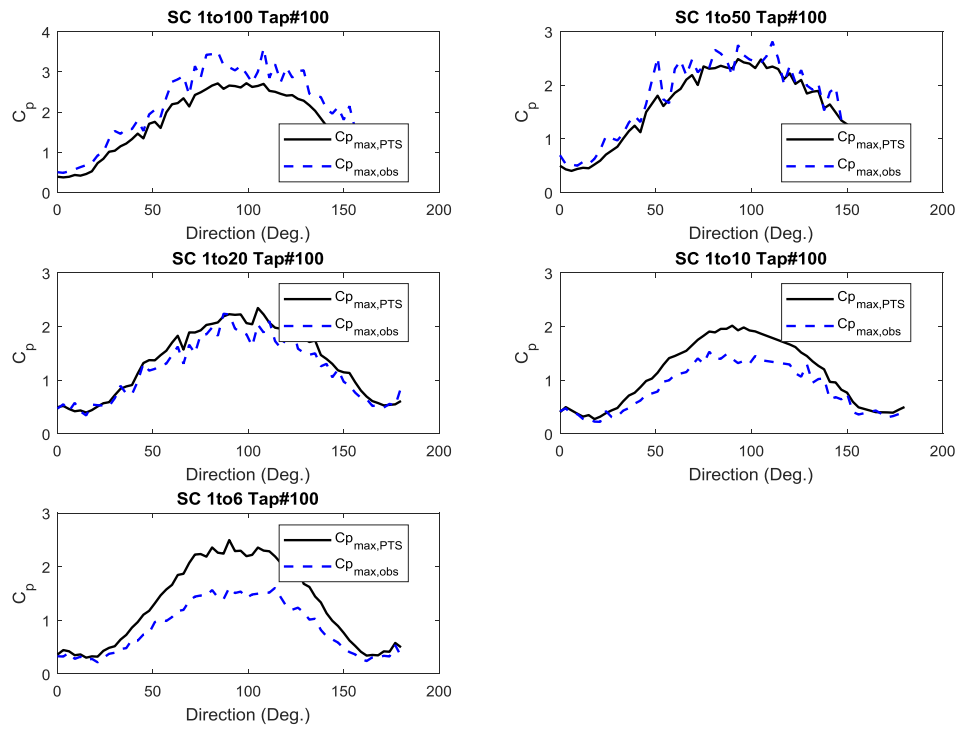

Figure 75 - Observed peaks vs. PTS peaks, wall tap\#100
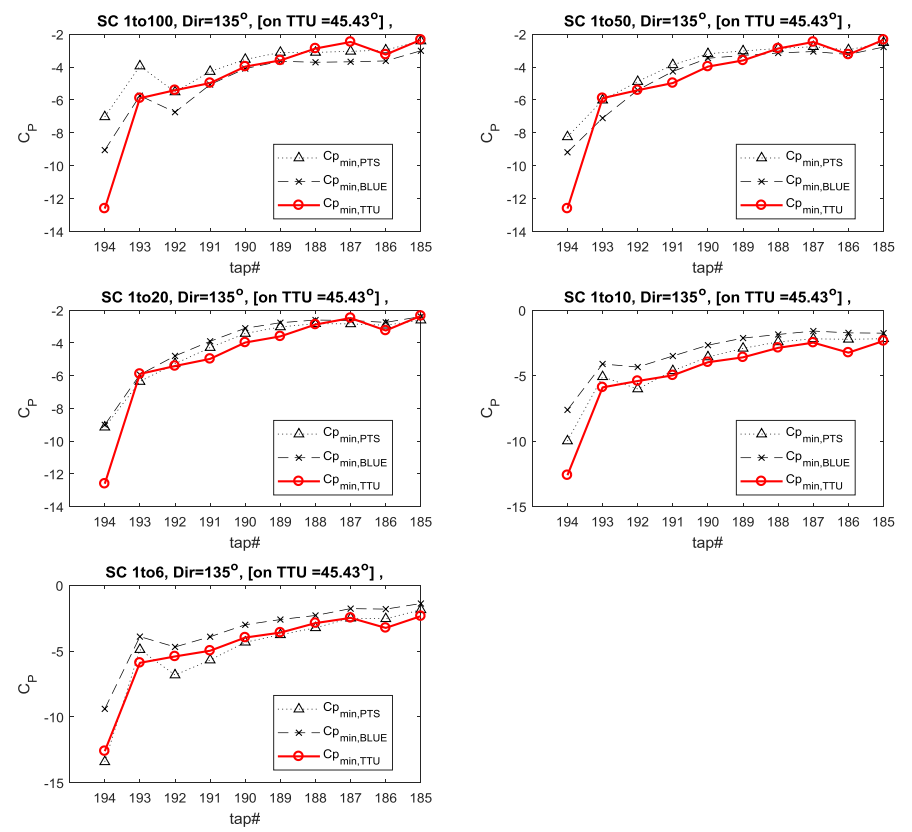

Figure 76 - PTS vs. EV I (BLUE) roof taps 

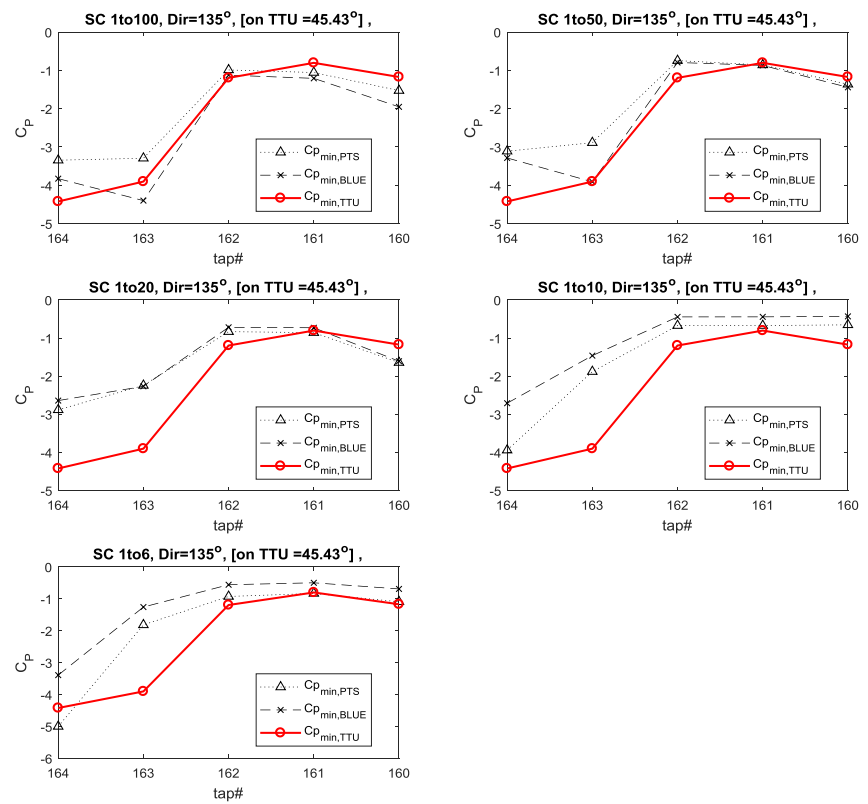

Figure 77 - PTS vs. EV I (BLUE) roof taps

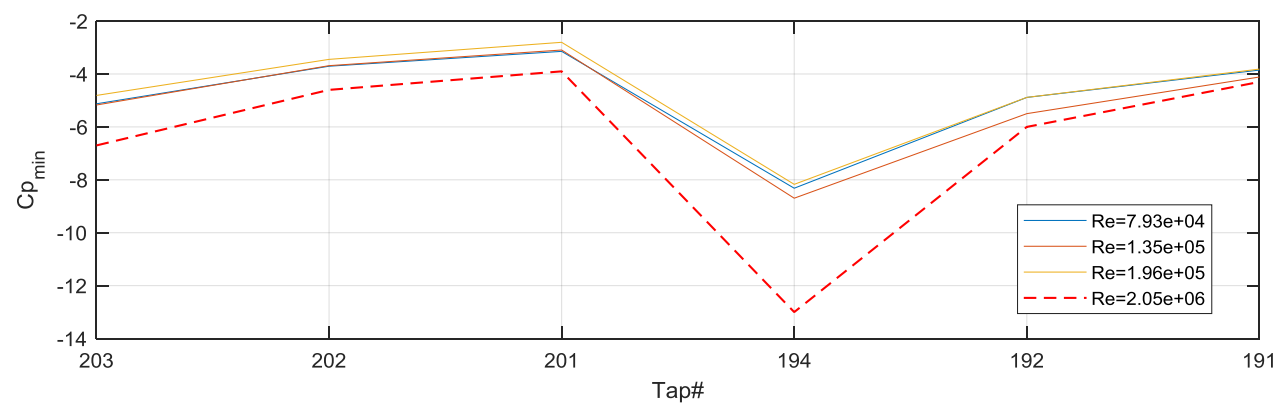

Figure 78 - Reynolds number effect on $\mathrm{C}_{\mathrm{p} \text { min }}$, Scale 1:50

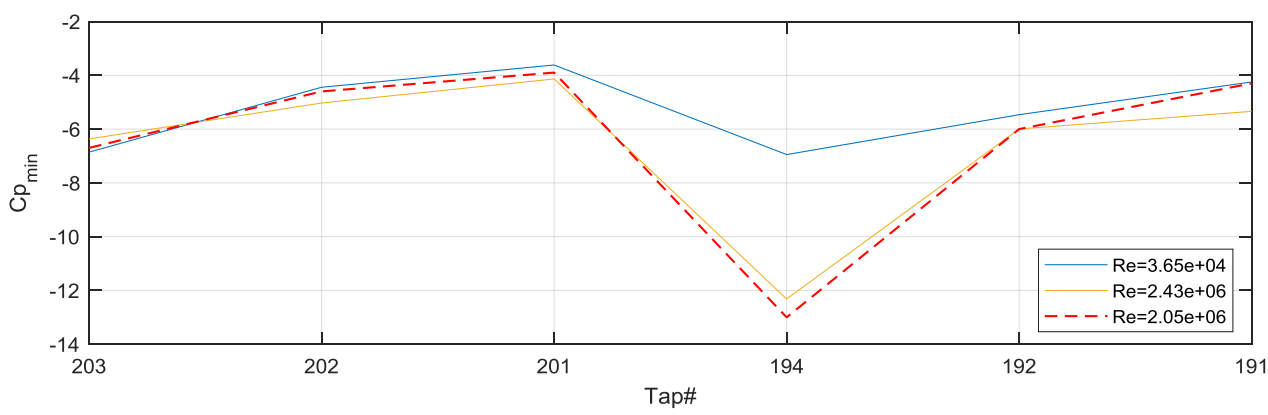

Figure 79 - Reynolds number effect, $C_{p \min }$, Scales 1:100 \& 1:6 


\section{CHAPTER V}

\section{SUMMARY \& CONCLUSIONS}

Wind tunnel testing has become the primary tool to estimate the wind loads on structures and buildings and to establish wind load provisions and standards, and along the way, the modeling techniques and data analysis procedures have also advanced continuously to address the emerging challenges. One of the challenges with a history of research and scientific discussions has been the large-scale modeling of the low-rise buildings.

The turbulent wind flow is comprised of eddies of various sizes, from the very small ones to the eddies as large as hundred times the building dimensions. The average size of those eddies is represented by a flow characteristic parameter named turbulence integral length scale. As the model size increases, relatively less large-scale turbulence in the upcoming flow can be generated, and it gets increasingly difficult to maintain scaling for the integral length scale of atmospheric turbulence to be same as the scale used to model the building as for larger models. Since the large-scale turbulence (large eddies) fluctuate at lower frequencies, the resulting turbulence power spectra show a lack of low-frequency turbulence content. This deficiency is known to have significant effects on the estimated peak wind loads. To overcome these limitations, the method of Partial Turbulence Simulation (PTS) has been recently developed by the FIU Wall of Wind lab to analytically compensate for the effects of the missing low-frequency part of the spectrum in the posttest analysis using quasi-steady assumptions. The current study was an effort to complement and enhance that method by investigating the effects of scaling and the range of applicability of the method, considering the limitations risen from the underlying theory, and to simplify the 2DPTS by proposing a weighted average approach. The two 
dimensional PTS incorporates both longitudinal and lateral components of the turbulence intensity in the peak estimation process which entails the numerical solution of a double integral, while the weighted average works with the longitudinal component of the turbulence and the effect of the lateral turbulence in incorporated via a weighting function derived from the probability distribution of the lateral turbulence around the target angle. Reynolds number effects was another objective of the study.

The comparisons between the field data and the results of the weighted average method, 1DPTS (or simply PTS, as denoted through out the text) and the 2DPTS, showed a good agreement between the weighted PTS and the 2DPTS for most points. Considering the 1DPTS, it could be seen that at the non-critical locations (i.e., locations with lower magnitude peaks) even the one dimensional PTS was performing well. But at the points within the separation bubble or the conical vortices, where highest magnitudes of peaks were observed, the difference was more considerable. This can be related to the constructive role of the lateral fluctuations of the flow in the formation mechanism of those phenomena. These comparison results indicate that the weighted average PTS method can be used in lieu of the 2DPTS approach

The results from five tested model scales showed as the model size was increased, a better agreement was achieved between the results of PTS peak estimation and the available field data from TTU building. Although at the smaller models $(1: 100,1: 50)$ almost a full range of turbulence spectrum was present, the highest peaks observed at fullscale couldn't be obtained which was speculated to be the effect of the Reynolds number mismatch between models and full-scale. The most accurate results were obtained when the PTS was applied to estimate the peaks from the model with the highest Reynolds 
number which was the biggest model (1:6 scale). Because of the large test section of the WOW, the blockage for this largest model still was less than $5 \%$ as it is recommended by the ASCE49-12[65] and the supporting literature.

Besides PTS, the peaks were also evaluated using the type I extreme value analysis (Gumbel fit). The mode and dispersion parameters of the fit were calculated by the Lieblein BLUE method. Those results also confirmed that at the smaller models where the similarity of the Reynolds number is considerably violated, the existence of a full-range turbulence spectrum is not sufficient to reproduce the highest peaks observed at full-scale. The ${ }^{x} L_{u m} / b_{m}$ ratio for the 1:6 model was 0.78 which is apparently smaller than the values recommended by the ASCE49-12, as it suggests the same ratio as the prototype (i.e., about 8 for the TTU building) or a value greater than 3 (to diminish the adverse effects of the missing lowfrequency turbulence and to ensure flow turbulence scales are larger than the building generated turbulence). This limit was only achieved for the 1:100 and 1:50 models in the study where almost a full turbulence spectrum was present.

So to achieve the most accurate results, a large-scale test followed by a simplified 2DPTS peak estimation method deemed to be the desirable approach which also allows the ${ }^{x} \mathrm{~L}_{\mathrm{um}} / \mathrm{b}_{\mathrm{m}}$ values much smaller than the ASCE recommended numbers, while still within the range of applicability of the quasi-steady assumptions. 


\section{CHAPTER VI}

\section{FUTURE WORK \& DEVELOPMENTS}

The effect of scaling on the partial turbulence simulation method was examined in detail and it was found that a ${ }^{\mathrm{x}} \mathrm{L}_{\mathrm{u}} / \mathrm{b}$ value as low as 0.8 still can be an acceptable scaling parameter as the PTS compensates the effect of the deficient low-frequency content of the generated turbulence and the larger dimension causes higher levels of Reynolds number. However, when large scales of taller prototypes are intended, such as mid-rise or high-rise buildings, the ${ }^{x} \mathrm{~L}_{\mathrm{u}} / \mathrm{b}$ proportion may take even a smaller value. Since valuable field data from fullscale studies of TTU building and Silsoe cube data are available as a verification tool, and with the advantage of having a large test section at WOW, it will be useful to investigate one model larger than 1:6 to examine the lower bounds of ${ }^{\mathrm{x}} \mathrm{L}_{\mathrm{u}} / \mathrm{b}$. Also to explore the range of adequacy for the Reynolds number, it will be useful to study the 1:10 model at higher speeds to add a midpoint to the data obtained from 1:100 and 1:6 models as the far ends of the range. Another beneficial study will be to apply PTS to the data available from other wind tunnels, where a scaled model of the TTU building has been studied. 


\section{REFERENCES}

[1] P. Irwin, "Bluff body aerodynamics in wind engineering.," Eng Ind Aerodyn, vol. 96, pp. 701-12, 2008.

[2] A. M. Aly, "Atmospheric boundary-layer simulation for the built environment: Past, present and future," Build. Environ., vol. 75, pp. 206-221, May 2014.

[3] J. E. Cermak, "Wind-tunnel development and trends in applications to civil engineering," J. Wind Eng. Ind. Aerodyn., vol. 91, no. 3, pp. 355-370, Feb. 2003.

[4] T. Stathopoulos, "The numerical wind tunnel for industrial aerodynamics: Real or virtual in the new millennium?," Wind Struct., vol. 5, no. 2_3_4, pp. 193-208, 2002.

[5] P. Irwin, "The role of wind tunnel modelling in the prediction of wind effects on bridges," presented at the Theinternationalsymposiumonadvancesin bridge aerodynamics, 1998.

[6] P. A. Irwin, "Wind Engineering Research Needs, Building Codes and Project Specific Studies," p. 14.

[7] M. Moravej, I. Zisis, A. G. Chowdhury, P. Irwin, and B. Hajra, "Experimental Assessment of Wind Loads on Vinyl Wall Siding," Front. Built Environ., vol. 2, p. 35, Dec. 2016.

[8] A. D. Cope et al., "Wind Loads on Components of Multi-Layer Wall Systems with Air-Permeable Exterior Cladding," 2012, pp. 238-257.

[9] R. A. V. Straaten, "Pressure Equalization of Wind-Induced Pressures on Residential Vinyl Siding Cladding in Full-Scale," p. 146.

[10] T. Stathopoulos and D. Surry, "Scale effects in wind tunnel testing of low buildings," J. Wind Eng. Ind. Aerodyn., vol. 13, no. 1-3, pp. 313-326, Dec. 1983.

[11] M. Jensen and N. Franck, "Model-scale tests in turbulent wind part 2. Phenomena dependent on the velocity pressure," Danish Technical press, 1965.

[12] N. J. Cook, "Determination of the model scale factor in wind-tunnel simulations of the adiabatic atmospheric boundary layer," J. Wind Eng. Ind. Aerodyn., vol. 2, no. 4, pp. 311-321, Jan. 1978.

[13] J. C. K. Cheung, J. D. Holmes, W. H. Melbourne, N. Lakshmanan, and P. Bowditch, "Pressures on a 1:10 scale model of the Texas Tech Building," J. Wind Eng. Ind. Aerodyn., vol. 69-71, no. Supplement C, pp. 529-538, Jul. 1997.

[14] H. W. Tieleman, "Wind tunnel simulation of wind loading on low-rise structures: a review," J. Wind Eng. Ind. Aerodyn., vol. 91, no. 12-15, pp. 1627-1649, Dec. 2003.

[15] R. Hillier and N. J. Cherry, "The effects of stream turbulence on separation bubbles," J. Wind Eng. Ind. Aerodyn., vol. 8, no. 1, pp. 49-58, Jul. 1981.

[16] W. H. MELBOURNE, “TURBULENCE EFFECTS ON MAXIMUM SURFACE PRESSURES - A MECHANISM AND POSSIBILITY OF REDUCTION," in Wind Engineering, J. E. CERMAK, Ed. Pergamon, 1980, pp. 541-551.

[17] A. Hunt, "scale effects on wind tunnel measurements of surface pressures on model buildings," in Designing with the Wind, Nantes, France, 9181.

[18] M. Jensen and N. Franck, Model-scale tests in turbulent wind. Copenhagen: Danish Technical Press, 1963. 
[19] Z. Y. Wang, E. J. Plate, M. Rau, and R. Keiser, "Scale effects in wind tunnel modelling," J. Wind Eng. Ind. Aerodyn., vol. 61, no. 2-3, pp. 113-130, Jul. 1996.

[20] P. J. Richards, R. P. Hoxey, and L. J. Short, "Wind pressures on a $6 \mathrm{~m}$ cube," J. Wind Eng. Ind. Aerodyn., vol. 89, no. 14, pp. 1553-1564, 2001.

[21] P. J. Richards, R. . Hoxey, B. D. Connell, and D. P. Lander, "Wind-tunnel modelling of the Silsoe Cube," J. Wind Eng. Ind. Aerodyn., vol. 95, no. 9-11, pp. 1384-1399, Oct. 2007.

[22] M. Kasperski and R. Hoxey, "Extreme-value analysis for observed peak pressures on the Silsoe cube," J. Wind Eng. Ind. Aerodyn., vol. 96, no. 6, pp. 994-1002, Jun. 2008.

[23] P. Richards and S. Norris, "LES modelling of unsteady flow around the Silsoe cube," J. Wind Eng. Ind. Aerodyn., vol. 144, pp. 70-78, Sep. 2015.

[24] G. M. Richardson and D. Surry, "The Silsoe Building: a comparison of pressure coefficients and spectra at model and full-scale," J. Wind Eng. Ind. Aerodyn., vol. 43, no. 1, pp. 1653-1664, Jan. 1992.

[25] M. L. Levitan and K. C. Mehta, "Texas Tech field experiments for wind loads part 1: building and pressure measuring system," J. Wind Eng. Ind. Aerodyn., vol. 43, no. 1, pp. 1565-1576, Jan. 1992.

[26] M. L. Levitan and K. C. Mehta, "Texas tech field experiments for wind loads part II: meteorological instrumentation and terrain parameters," J. Wind Eng. Ind. Aerodyn., vol. 43, no. 1, pp. 1577-1588, Jan. 1992.

[27] L. Cochran and J. Cermak, "Full-and model-scale cladding pressures on the Texas Tech University experimental building.," Eng Ind Aerodyn, vol. 43, pp. 1589-600, 1992.

[28] B. D. Lakas, K. C. Mehta, E. W. Kiesling, and J. R. Dunn, "Wind tunnel simulation of the Texas Tech research building: Effects of terrain features and building orientation," J. Wind Eng. Ind. Aerodyn., vol. 36, pp. 1073-1082, Jan. 1990.

[29] M. L. Levitan, K. C. Mehta, W. P. Vann, and J. D. Holmes, "Field measurements of pressures on the Texas tech building," J. Wind Eng. Ind. Aerodyn., vol. 38, no. 2-3, pp. 227-234, Jul. 1991.

[30] K. C. Mehta, M. L. Levitan, R. E. Iverson, and J. R. McDonald, "Roof corner pressures measured in the field on a low building," J. Wind Eng. Ind. Aerodyn., vol. 41, no. 1, pp. 181-192, Oct. 1992.

[31] S. A. Bekele and H. Hangan, "A comparative investigation of the TTU pressure envelope -Numerical versus laboratory and full scale results," Wind Struct., vol. 5, no. 2_3_4, pp. 337-346, 2002.

[32] A. Mochida, S. Murakami, M. Shoji, and Y. Ishida, "Numerical Simulation of flowfield around Texas Tech Building by Large Eddy Simulation," J. Wind Eng. Ind. Aerodyn., vol. 46-47, pp. 455-460, Aug. 1993.

[33] R. Panneer Selvam and P. B. Konduru, "Computational and experimental roof corner pressures on the Texas Tech Building," J. Wind Eng. Ind. Aerodyn., vol. 46-47, pp. 449-454, Aug. 1993.

[34] J. He and C. C. S. Song, "A numerical study of wind flow around the TTU building and the roof corner vortex," J. Wind Eng. Ind. Aerodyn., vol. 67-68, pp. 547-558, Apr. 1997. 
[35] "NUMERICAL SIMULATION STUDY OF 3D STEADY ATMOSPHERIC FLOW AROUND STANDARD LOW-RISE BUILDING TTU MODEL-- 《Engineering Mechanics》2007年02期.” http://en.cnki.com.cn/Article_en/CJFDTOTAL-GCLX200702025.htm. [Accessed: 21-Jun-2018].

[36] H. W. Tieleman, D. Surry, and K. C. Mehta, "Full/model-scale comparison of surface pressures on the Texas Tech experimental building," J. Wind Eng. Ind. Aerodyn., vol. 61, no. 1, pp. 1-23, Jun. 1996.

[37] H. Okada and H. Kikitsu, "Evaluation of wind resistance performance of clay tile roof based on survey of construction method and pulling-up tests.," Constr Eng 596, pp. 9-16, 2005.

[38] D. Surry, G. Kopp, and F. Bartlett, "Wind load testing of low buildings to failure at model and full scale.," Nat Hazards Rev, vol. 6, pp. 121-8, 2005.

[39] B. Bienkiewicz and Y. Sun, "Local wind loading on the roof of a low-rise building," J. Wind Eng. Ind. Aerodyn., vol. 45, no. 1, pp. 11-24, Dec. 1992.

[40] G. A. Kopp, M. J. Morrison, and D. J. Henderson, "Full-scale testing of low-rise, residential buildings with realistic wind loads," J. Wind Eng. Ind. Aerodyn., vol. 104106, pp. 25-39, May 2012.

[41] S. Ahmad and K. Kumar, "Interference effects on wind loads on low-rise hip roof buildings," Eng. Struct., vol. 23, no. 12, pp. 1577-1589, Dec. 2001.

[42] H. W. Tieleman, "Model/full scale comparison of pressures on the roof of the TTU experimental building," J. Wind Eng. Ind. Aerodyn., vol. 65, no. 1, pp. 133-142, Dec. 1996.

[43] S. Ahmad, "WIND PRESSURES ON LOW-RISE HIP ROOF BUILDINGS," 2000.

[44] M. Endo, B. Bienkiewicz, and H. J. Ham, "Wind-tunnel investigation of point pressure on TTU test building," J. Wind Eng. Ind. Aerodyn., vol. 94, no. 7, pp. 553578, Jul. 2006.

[45] Rofail A.W., "Full-Scale/Model-Scale Comparisons of Wind Pressures on The TTU Building," presented at the International conference; 9th, Wind engineering: retrospect and prospect, New Delhi, 1995.

[46] Murray J. Morrison, Tanya M. Brown, and Zhuzhao Liu, "Comparison of Field and Full-Scale Laboratory Peak Pressures at the IBHS Research Center," Adv. Hurric. Eng., 2013.

[47] J.-X. Lin, D. Surry, and H. W. Tieleman, "The distribution of pressure near roof corners of flat roof low buildings," J. Wind Eng. Ind. Aerodyn., vol. 56, no. 2, pp. 235-265, May 1995.

[48] M. Asghari Mooneghi, "Experimental and Analytical Methodologies for Predicting Peak Loads on Building Envelopes and Roofing Systems," Florida Int'l University, FIU Electronic Theses and Dissertations. Paper 1846, 2014.

[49] M. Asghari Mooneghi, P. Irwin, and A. Gan Chowdhury, "Partial turbulence simulation method for predicting peak wind loads on small structures and building appurtenances," J. Wind Eng. Ind. Aerodyn., vol. 157, pp. 47-62, Oct. 2016.

[50] C. W. Letchford and K. C. Mehta, "The distribution and correlation of fluctuating pressures on the Texas Tech Building," J. Wind Eng. Ind. Aerodyn., vol. 50, pp. 225234, Dec. 1993. 
[51] M. Moravej, C. Arindam, and I. Peter, "FMGLOBAL Project \# WOW-2015-057."

[52] C. W. Letchford, R. E. Iverson, and J. R. McDonald, "The application of the Quasisteady Theory to full scale measurements on the Texas Tech building," J. Wind Eng. Ind. Aerodyn., vol. 48, no. 1, pp. 111-132, Sep. 1993.

[53] C. R. Chu, M. B. Parlange, G. G. Katul, and J. D. Albertson, "Probability density functions of turbulent velocity and temperature in the atmospheric surface layer," Water Resour. Res., vol. 32, no. 6, pp. 1681-1688, Jun. 1996.

[54] "IHS ESDU 85020: Atmospheric turbulence near ground: data for winds." [Online]. Available: https://www.esdu.com/cgibin/ps.pl?sess=unlicensed_1180429191652ynt\&t $=$ doc\&p=esdu_85020g. [Accessed: 29-Apr-2018].

[55] H. Kawai, "PRESSURE FLUCTUATIONS ON SQUARE PRISMS APPLICABILITY OF STRIP AND QUASI-STEADY THEORIES," in Wind Engineering 1983, J. D. Holmes, P. S. Jackson, and W. H. Melbourne, Eds. Elsevier, 1984, pp. 197-208.

[56] A. Gan Chowdhury et al., "Large-Scale Experimentation Using the 12-Fan Wall of Wind to Assess and Mitigate Hurricane Wind and Rain Impacts on Buildings and Infrastructure Systems," J. Struct. Eng., vol. 143, no. 7, p. 04017053, Jul. 2017.

[57] H. P. A. H. Irwin, K. R. Cooper, and R. Girard, "Correction of distortion effects caused by tubing systems in measurements of fluctuating pressures," J. Wind Eng. Ind. Aerodyn., vol. 5, no. 1, pp. 93-107, Oct. 1979.

[58] P. A. Irwin, "Exposure categories and transitions for design wind loads," J. Struct. Eng., vol. 132, no. 11, pp. 1755-1763, 2006.

[59] H. Lettau, "Note on Aerodynamic Roughness-Parameter Estimation on the Basis of Roughness-Element Description," J. Appl. Meteorol., vol. 8, no. 5, pp. 828-832, Oct. 1969.

[60] Z. Zhao, "Wind flow characteristics and their effects on low-rise buildings," Dec. 1997.

[61] T. C. E. Ho, D. Surry, and D. Morrish, "NIST/TTU Cooperative Agreement Windstorm Mitigation Initiative: Wind Tunnel Experiments on Generic Low Buildings," BLWT-SS20-2003, May 2003.

[62] T. C. E. Ho, D. Surry, D. Morrish, and G. A. Kopp, "The UWO contribution to the NIST aerodynamic database for wind loads on low buildings: Part 1. Archiving format and basic aerodynamic data," J. Wind Eng. Ind. Aerodyn., vol. 93, no. 1, pp. 1-30, Jan. 2005.

[63] J. Lieblein, "Efficient methods of extreme-value methodology," National Bureau of Standards, Gaithersburg, MD, NBS IR 74-602, 1974.

[64] A. M. Aly, A. G. Chowdhury, and G. Bitsuamlak, "Wind profile management and blockage assessment for a new 12-fan Wall of Wind facility at FIU," Wind Struct., vol. 14, no. 4, pp. 285-300, 2011.

[65] "Wind Tunnel Testing for Buildings and Other Structures (49-12)| ASCE." ASCE, 2012. 


\section{APPENDICES}

\subsection{PTS Application Procedure: Step by Step Example}

In this section the step by step computations for the PTS peak estimation of the tap 202 (Figure 10) of the 1:6 model is demonstrated. This tap is located on the edge of the roof, and the pressure data collected at the wind attack angle of $135^{\circ}$ is used.

The steps are as follows:

1) Some statistics of the pressure data:

\begin{tabular}{|l|l|}
\hline & Pressure (psi) \\
\hline Tap No; & 202 \\
\hline Mean & -0.07172 \\
\hline Max & -0.02661 \\
\hline Min & -0.14988 \\
\hline
\end{tabular}

2) Time history of the pressures is converted into a time history of pressure coefficients (table shows the observed values)

\begin{tabular}{|l|l|}
\hline & Cp time history \\
\hline Tap No; & 202 \\
\hline Cp mean & -1.88141 \\
\hline Cp max & -0.69819 \\
\hline Cp min & -3.93183 \\
\hline
\end{tabular}

3) Model and full-scale parameters:

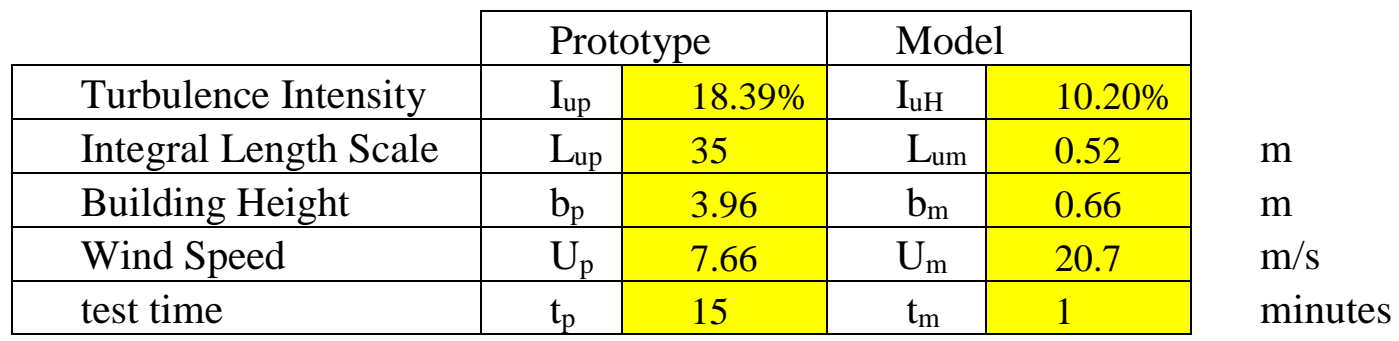


Checking the ratio of model turbulence intensity to prototype turbulence intensity: as discussed in Chapter 2, Eq. 8 (repeated here for more convenience) should hold true to ensure a match at the high end of the turbulence power spectra. [49]

$$
\frac{I_{u m}}{I_{u p}}=\left(\frac{{ }^{x} L_{u m}}{{ }^{x} L_{u p}}\right)^{1 / 3}\left(\frac{b_{p}}{b_{m}}\right)^{1 / 3}
$$

\begin{tabular}{|l|l|}
\hline $\mathrm{I}_{\mathrm{um}} / \mathrm{I}_{\mathrm{up}}=$ & 0.47 \\
\hline $\mathrm{L}_{\mathrm{um}} / \mathrm{L}_{\mathrm{up}}=$ & 0.014 \\
\hline $\mathrm{b}_{\mathrm{p}} / \mathrm{b}_{\mathrm{m}}=$ & 6 \\
\hline$\left(\mathrm{L}_{\mathrm{um}} / \mathrm{L}_{\mathrm{up}}\right)^{1 / 3} \times\left(\mathrm{b}_{\mathrm{p}} / \mathrm{b}_{\mathrm{m}}\right)^{1 / 3}=$ & 0.45 \\
\hline
\end{tabular}

$I_{u m}$ is equal to $I_{u H}$, the longitudinal turbulence intensity at the model eave height.

4) Calculating PTS parameters:

\begin{tabular}{|c|c|c|}
\hline $\mathrm{I}_{\mathrm{uL}}$ & $15.30 \%$ & $I_{u L}=\sqrt{I_{u}^{2}-I_{u H}^{2}}$ \\
\hline $\mathrm{U}_{\mathrm{Lp}}$ & 11.645 & $\hat{U}_{L p}=U_{p}\left(1+3.4 I_{u L}\right)$ \\
\hline $\mathrm{Um} / \mathrm{U}_{\mathrm{Lp}}$ & 1.778 & $\lambda_{U}=\frac{U_{m}}{\widehat{U}_{L p}}$ \\
\hline $\mathrm{nm} / \mathrm{np}$ & 10.665 & $\lambda_{n}=\lambda_{U} \frac{b_{p}}{b_{m}}$ \\
\hline $\mathrm{tm} / \mathrm{tp}$ & 0.094 & $\lambda_{t}=1 / \lambda_{n}$ \\
\hline Dividing frequency & 0.092 & $n_{c}=0.0716 \frac{U}{{ }^{x} L_{u}}\left(\frac{I_{u}}{I_{u H}}\right)^{3}$ \\
\hline$t_{\text {Gust.p }}$ & 4.900 & $t_{\text {gust }}=\frac{0.45}{n_{c}}$ \\
\hline$t_{\text {Gust, } m}$ & 0.459 & $\mathrm{t}_{\text {Gust }, \mathrm{m}}=\mathrm{t}_{\text {Gust, } \mathrm{p}} \times \lambda_{\mathrm{t}}$ \\
\hline $\max \mathrm{N}$ & 131 & $=\mathrm{t}_{\mathrm{m}} / \mathrm{t}_{\mathrm{Gust}, \mathrm{m}}$ \\
\hline $\mathrm{N}=$ & 100 & $\begin{array}{l}\text { Should be less than "max N" (or } \\
\text { each subinterval duration should be } \\
\text { more than the gust duration }\end{array}$ \\
\hline$t_{\text {sub, }, \mathrm{m}}$ & 0.6 & $>\mathrm{t}_{\mathrm{Gust}, \mathrm{m}, \mathrm{OK}}$ \\
\hline$t_{\text {sub,p }}$ & 6.4 & \\
\hline $\mathrm{Gi}=$ & 0.00711 & $=\frac{t_{\text {subinterval }, p}}{\text { Full Scale time }(\text { e.g. } 3600 \mathrm{sec})}$ \\
\hline
\end{tabular}


5) Dividing time series of pressure coefficients into 100 subintervals and calculate the maximum and minimum of each subinterval (first 10 subintervals are illustrated in the following table):

\begin{tabular}{|r|rr|r|}
\hline \multicolumn{1}{|c|}{$\begin{array}{l}\text { Interval } \\
\text { No; }\end{array}$} & $\begin{array}{c}\text { Min Cp } \\
\text { subinterval }\end{array}$ & of the & Max Cp of the subinterval \\
\hline 1 & -3.55631 & -1.23625 \\
\hline 2 & -2.82251 & -1.06414 \\
\hline 3 & -3.70617 & -1.06089 \\
\hline 4 & -2.79941 & -1.09363 \\
\hline 5 & -3.20297 & -0.75349 \\
\hline 6 & -2.68242 & -0.92059 \\
\hline 7 & -2.78025 & -1.04141 \\
\hline 8 & -2.49432 & -1.13299 \\
\hline 9 & -2.774 & -1.15961 \\
\hline 10 & -3.01444 & -1.10068 \\
\hline
\end{tabular}

6) To estimate the probability that the peak pressure coefficient will not exceed a value $\hat{C}$ in a subinterval, the subinterval peaks are fitted into a Fisher Tippet Type I distribution:

$$
\begin{aligned}
& p=F(\hat{C})=\exp (-\exp (-a(\hat{C}-b))) \\
& \ln p=-\exp (-a(\hat{C}-b)) \\
& \ln (-\ln p)=-a(\hat{C}-b) \\
& -\ln (-\ln p)=a \hat{C}-a b \\
& \widehat{\mathrm{C}}=\frac{1}{\mathrm{a}}(-\ln (-\ln \mathrm{p}))+\mathrm{b}
\end{aligned}
$$

Following table shows a part of the data used to do the fit $\left(\mathrm{C}_{\min }\right.$ column is sorted ascending, then to obtain the probability of exceedance the value of 1-p is used. 


\begin{tabular}{|r|r|r|r|}
\hline \multicolumn{1}{c|}{$\mathrm{n}$} & \multicolumn{1}{c|}{$\mathrm{C}_{\min }$} & $\mathrm{p}=\mathrm{n} /(\mathrm{N}+1)$ & $\mathrm{y}=-\mathrm{LN}(-\mathrm{LN}(1-\mathrm{p}))$ \\
\hline 1 & -3.93183 & 0.009804 & 4.620051 \\
\hline 2 & -3.86435 & 0.019608 & 3.921941 \\
\hline 3 & -3.8501 & 0.029412 & 3.511471 \\
\hline 4 & -3.83958 & 0.039216 & 3.218742 \\
\hline 5 & -3.82761 & 0.04902 & 2.990509 \\
\hline 6 & -3.74506 & 0.058824 & 2.803054 \\
\hline 7 & -3.72365 & 0.068627 & 2.643725 \\
\hline 8 & -3.70617 & 0.078431 & 2.50497 \\
\hline 9 & -3.63378 & 0.088235 & 2.381917 \\
\hline
\end{tabular}

$\mathrm{m}=1 / \mathrm{a}=-0.292, \mathrm{~b}=-2.93 \rightarrow \mathrm{a}=-3.42, \mathrm{ab}=10.02$

7) Solving the probability integral numerically to obtain the $C_{p}$ value at the desired probability level. See Chapter 2 (Eq. 14) for more details.

$$
G\left(\frac{\hat{C}_{p}}{b}\right)=\int_{-\infty}^{\infty} \frac{1}{\sqrt{2 \pi}} e^{-\frac{1}{2} \xi^{2}}\left[1-\exp \left(-\exp \left(-a b\left(\frac{\hat{C}_{p} / b}{\left(1+I_{u L} \xi\right)^{2}}-1\right)\right)\right)\right] d \xi
$$

Having $\mathrm{G}=0.00711$ and $\mathrm{ab}=10.024$, the corresponding $\mathrm{C}_{\mathrm{p}} / \mathrm{b}$ becomes 2.136 which yields $C_{p}=-6.26$. 


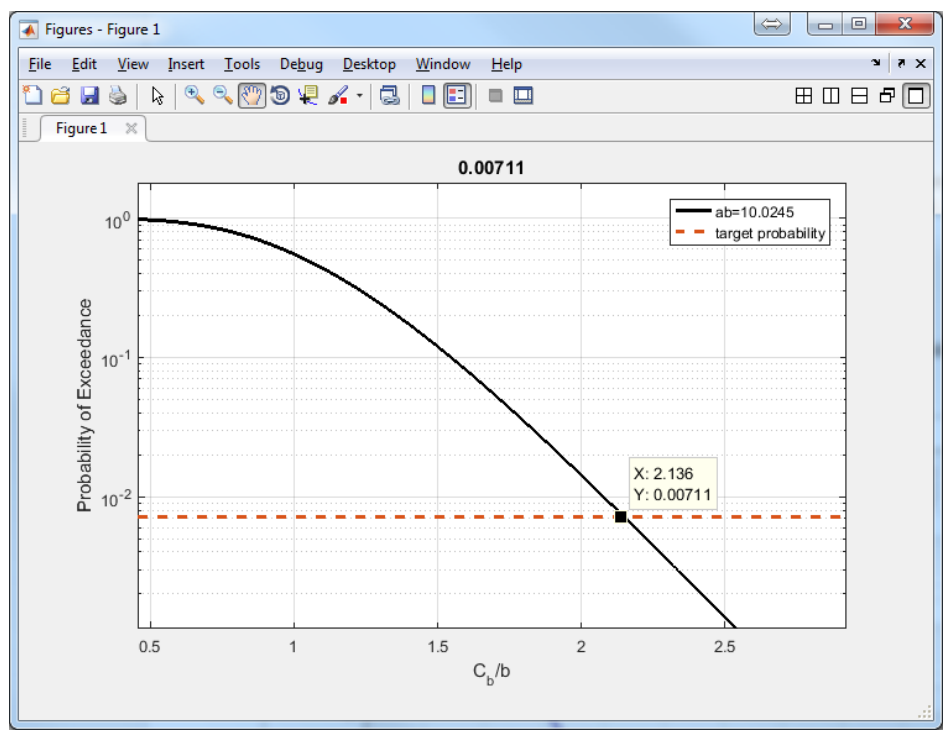

Figure 80 - Numerical solution of Eq. 14

The sensitivity of the method to the number of subintervals and also the changes in the value of $I_{u L}$ is shown in Figure 81 to Figure 85.

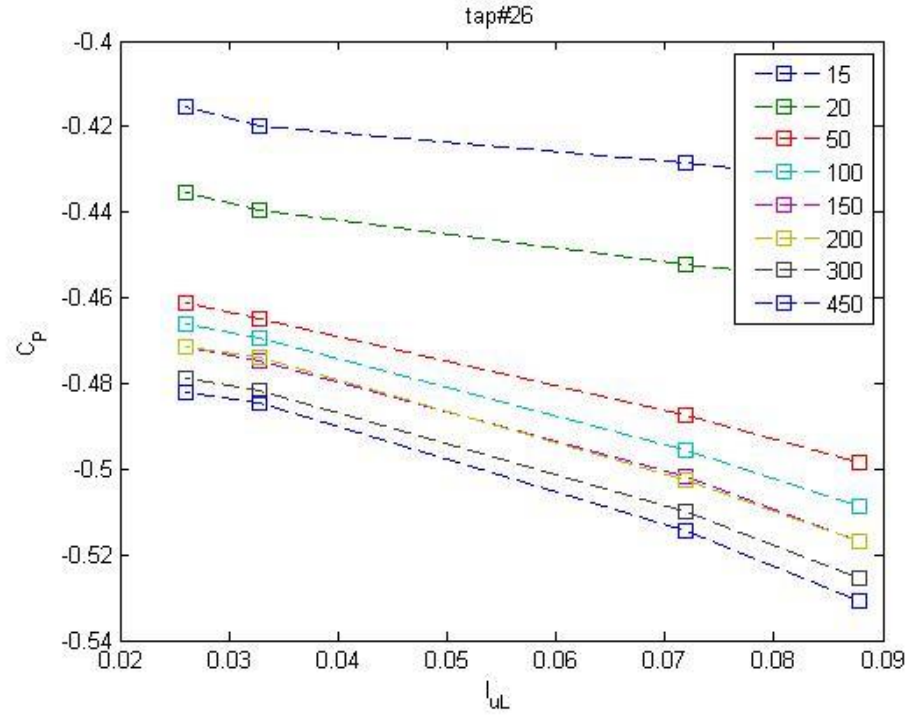

Figure $81-\mathrm{C}_{\mathrm{p}}$ vs. $\mathrm{I}_{\mathrm{uL}}$ at different $\mathrm{N}$ values, tap\#26, Scale 1:100 


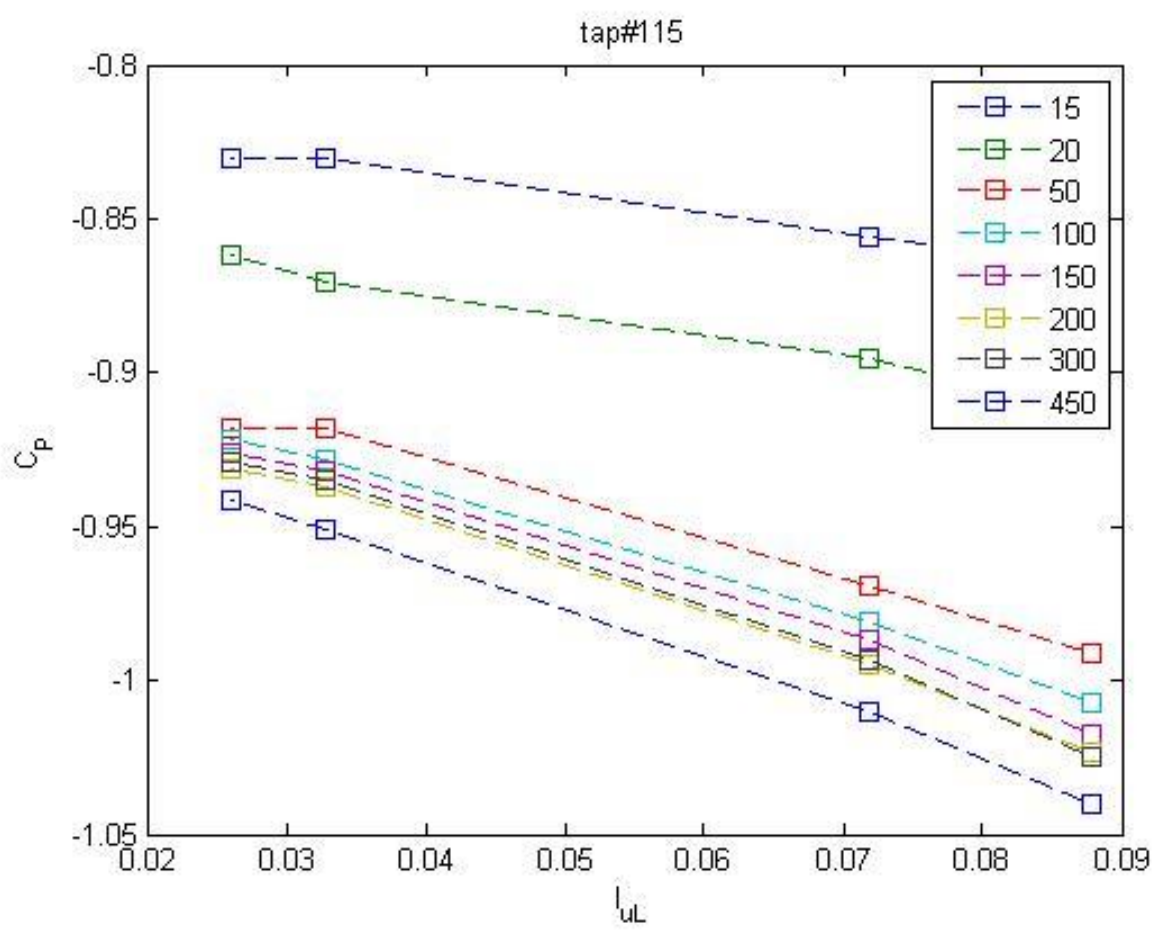

Figure $82-C_{p}$ vs. $\mathrm{I}_{u L}$ at different $\mathrm{N}$ values, tap\#115, Scale 1:100

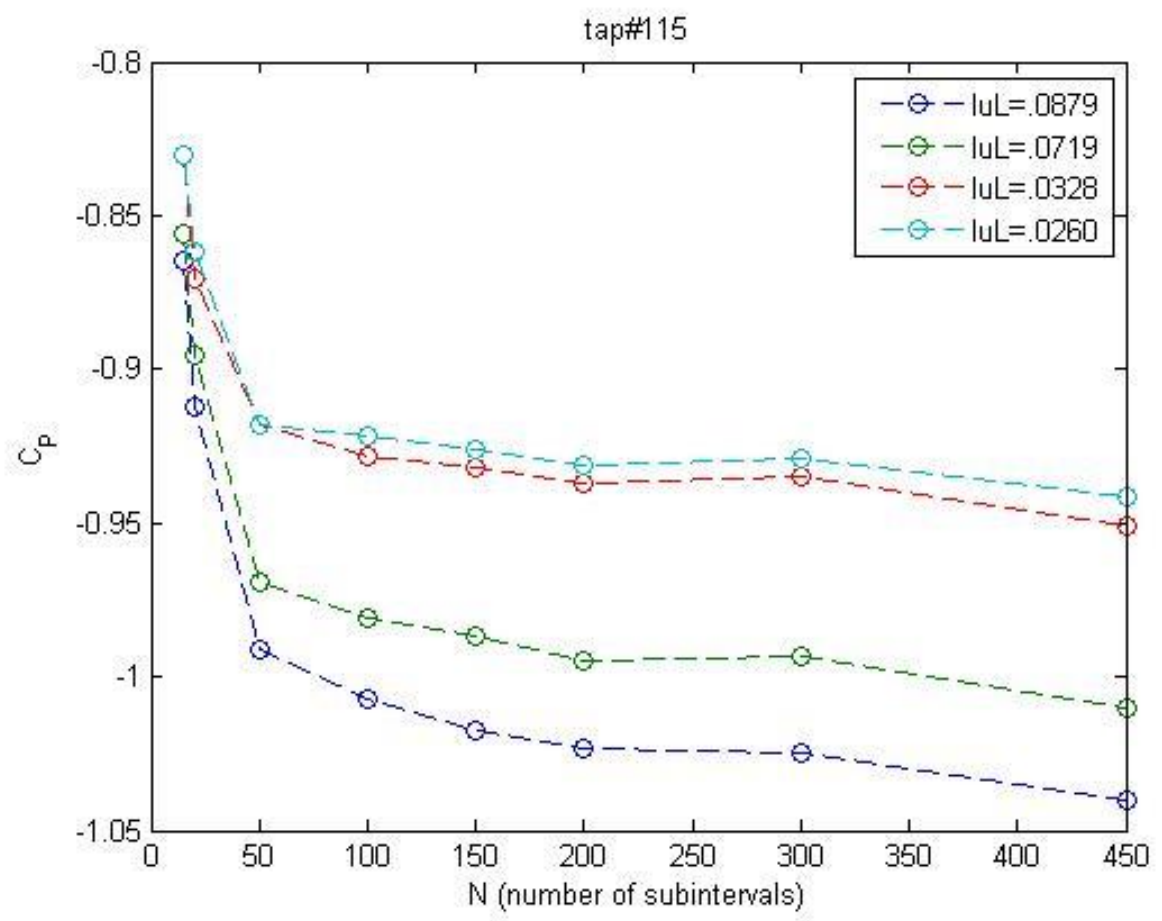

Figure $83-C_{p}$ vs. $N$ at different $I_{u L}$ values, tap\#115, Scale 1:100 


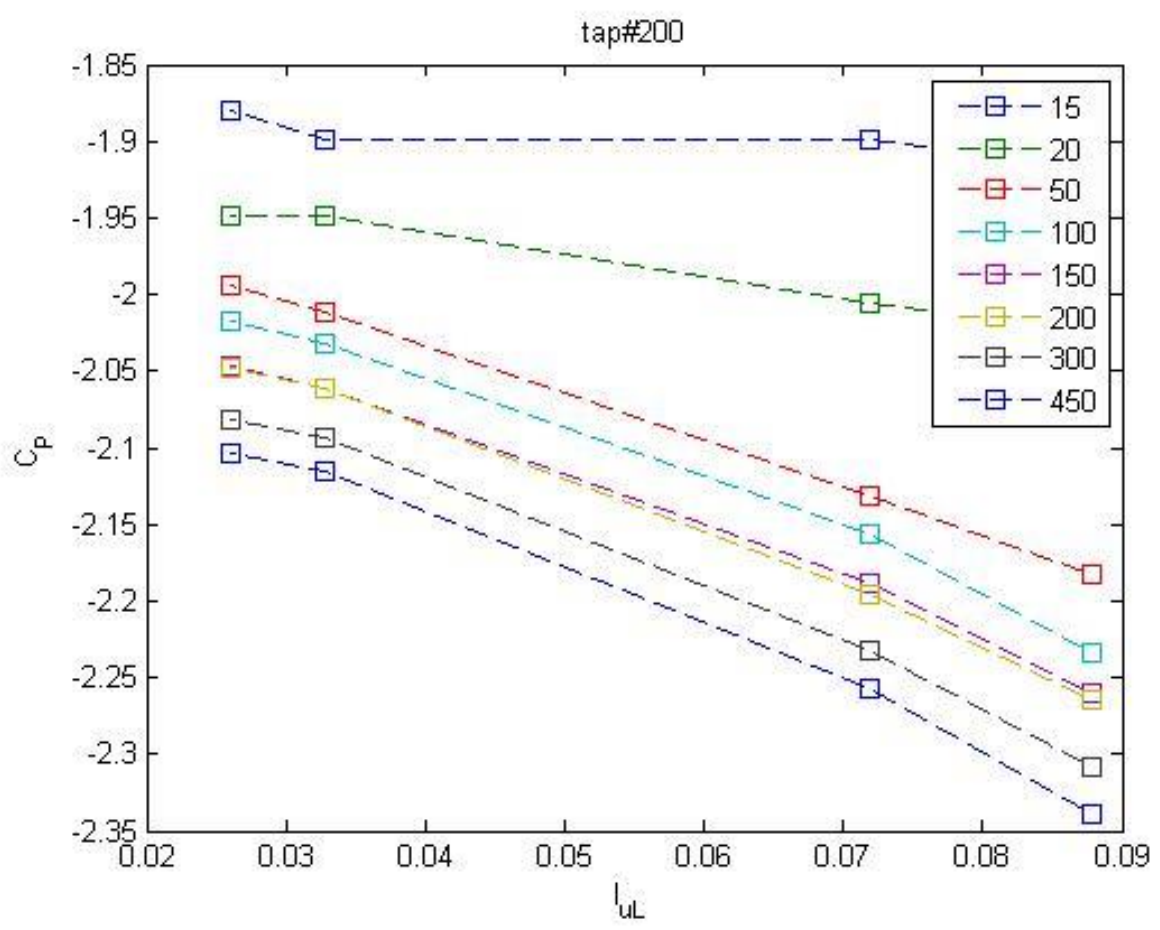

Figure $84-C_{p}$ vs. $I_{u L}$ at different $N$ values, tap\#200, Scale 1:100

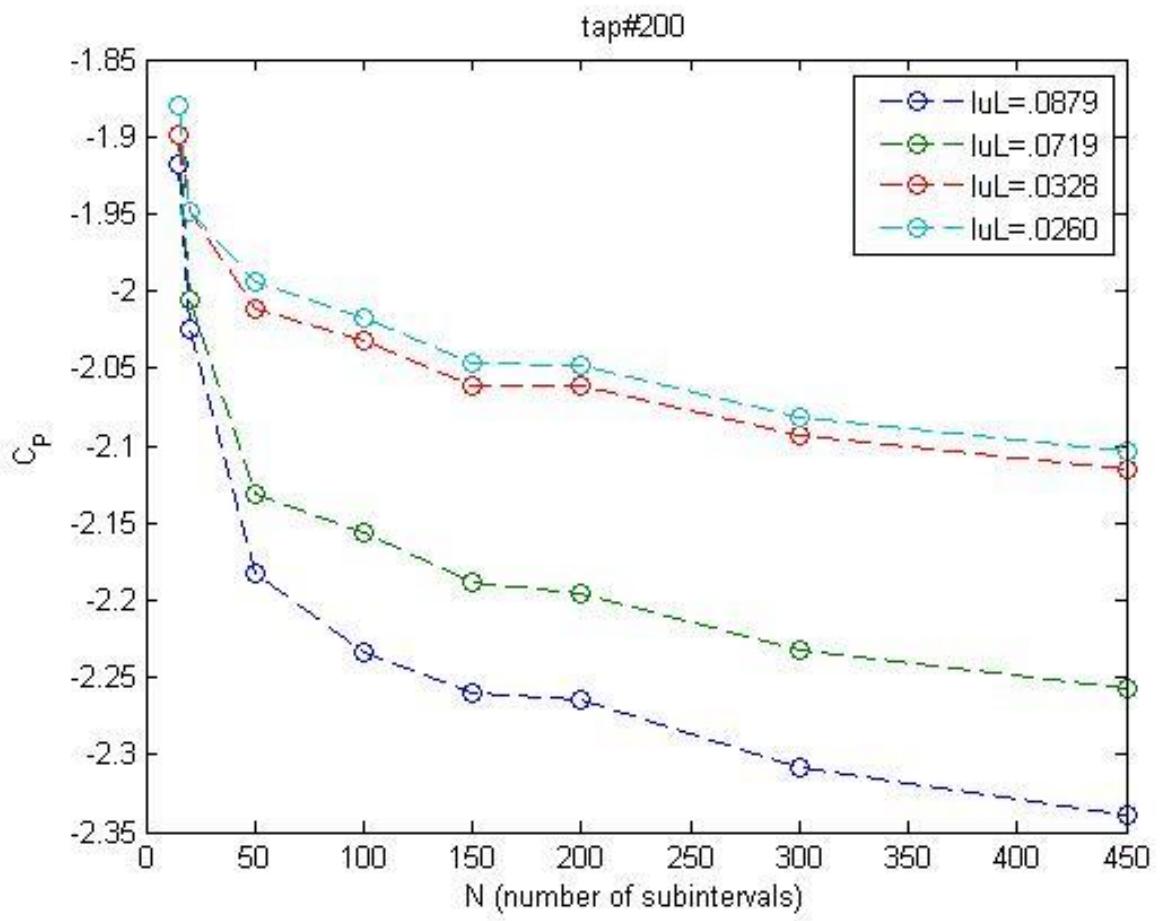

Figure $85-C_{p}$ vs. $N$ at different $I_{u L}$ values, tap\#200, Scale 1:100 


\subsection{PTS MATLAB Codes \& Application Procedure}

The PTS analysis package comprises of an excel sheet and several MATLAB files as described here:

1- PTS parameters calculation spreadsheet named pts_calculations.xlsx to calculate to necessary parameters needed to perform the PTS analysis as shown in Figure 86.

\begin{tabular}{|c|c|c|c|c|c|c|c|c|c|c|c|c|c|c|}
\hline & c & D & E & $\mathrm{F}$ & G & $\mathrm{H}$ & 1 & J & k & L & M & $\mathrm{N}$ & o & $\mathrm{p}$ \\
\hline & & Input Data & & & & & & & & & & & & \\
\hline & & Verification points & & & & & & & & & & & & \\
\hline & & & & & & & & & & & & & & \\
\hline & & & & & & & & & & & & & & \\
\hline & & Prototype & & & & & & & & & & & & \\
\hline & $\mathrm{TI}$ & Iu (Iup in Paper) & $21.60 \%$ & IuH & $19.73 \%$ & & & & & & & & & \\
\hline & Lu (m) & Lup & 35 & Lum & 0.26 & & & & & & & & & \\
\hline & H (Roof height)(m) & bp & 3.96 & $\mathrm{bm}$ & 0.0396 & & & & & & & & & \\
\hline & $\mathrm{V}(\mathrm{m} / \mathrm{s})$ & Up (Target mean veloc & 7.66 & Um & 12.225 & & & & & & & & & \\
\hline & test time (minutes) & $\mathrm{Tp}$ (See Note 1) & 15 & $\mathrm{Tm}$ & 1 & & & & & & & & & \\
\hline & & & & & & & & & & & & & & \\
\hline & IuL (Eq. 9) & $8.79 \%$ & & & & & & & & & & & & \\
\hline & ULp (Eq. 51) & 9.950 & & & & \multicolumn{3}{|c|}{ To verify for Eq. 17} & & & & & & \\
\hline & Um/ULp (Eq. 52) & 1.229 & & & & \multicolumn{7}{|c|}{ Representative charachteristic time for turbulence } & & \\
\hline io & $\mathrm{nm} / \mathrm{np}$ (Inverse of Eq. 53) & 122.869 & & & & $\mathrm{Lu} / \mathrm{U}$ & 4.57 & s & & & & & & \\
\hline io & $\mathrm{tm} / \mathrm{tp}(=\mathrm{np} / \mathrm{nm})$ & 0.008 & & & & \multicolumn{8}{|c|}{ The ratio of full scale sample time to turbulence charact. Time: } & \\
\hline & Cut-off Frequency (nc) (Eq. & 0.021 & & & & $\mathrm{TsU} / \mathrm{Lu}$ & 197 & & & & & & & \\
\hline e) & tgust,p (Eq. 54) (seconds) & 21.886 & & & & & & & & & & & & \\
\hline el & tgust, $\mathrm{m}$ (seconds) & 0.178 & & & & \multicolumn{9}{|c|}{ If we achieve same ratio on the model then we should have stable statistics: } \\
\hline nt & $\max \mathrm{N}$ & 337 & & & & Ts mode & should & $\mathrm{be}=$ & 4.18 & & & & & \\
\hline ?) & $\mathrm{N}=$ & 80 & & & & \multicolumn{5}{|c|}{ (we should sample at least Ts seconds) } & & & & \\
\hline & each sub time at model (se & 0.75 & & & & & & & & & & & & \\
\hline & each sub at full (seconds) & 92.15 & & & & & & & & & & & & \\
\hline & Gi (Eq. 55) & 0.102390965 & & \multicolumn{2}{|c|}{ Eq 18. on "ratios" tab } & & & & & & & & & \\
\hline 1) & Percentile & 0.8 & & & & & & & & & & & & \\
\hline & G (Eq. 31) & 0.022588847 & & & & & & & & & & & & \\
\hline
\end{tabular}

Figure 86 - Excel sheet to calculate PTS analysis basic parameters

The definition of the PTS parameters and calculation steps can be found in Chapter 2,

Appendix 1, and the paper by Mooneghi et al. [49].

2- The Matlab function for the PTS analysis is called ptsFunc.mat and the code is displayed in Figure 87, and the syntax is as follows:

[c1 c2 c3 c4]=ptsFunc(CpTimeHistory,ns,G,IuL,convf)

The program inputs are as follows:

CpTimeHistory: is the time history of the pressure coefficients of a single tap 
ns: number of subintervals (epochs)

$\mathrm{G}$ : the probability of non-exceedance for the estimated peak

IuL: missing low turbulence intensity

convf: the conversion factor to convert peak hourly to a 3-sec peak (usually calculated as convf $\left.=1+3 \times I_{u}\right)$.

and the four outputs are as follows:

$\mathrm{c1}$ : $\mathrm{C}_{\mathrm{pmax}}$ (hourly)

$\mathrm{c} 2: \mathrm{C}_{\mathrm{pmax}}(3-\mathrm{sec})$

c3: $\mathrm{C}_{\text {pmin }}$ (hourly)

$\mathrm{c} 4: \mathrm{C}_{\mathrm{pmin}}(3-\mathrm{sec})$

The names are arbitrary and any acceptable MATLAB variable names can be used.

It should be noted that the function works on a single-column data array (i.e., the data related to a specific tap). The ptsFunc code calls three other functions as sub-routines which are defined as follows:

subdivide.m function: this function (Figure 88) divides a given time history into $\mathrm{N}$ subintervals. When the length of the time series is not exactly divisible by the $\mathrm{N}$, then subintervals of different lengths should be expected to cover all the data.

gumbelfit.m function: this function (Figure 89) receives the list of maximums or minimums of all subintervals as the input and returns the extreme value type I parameters (location and scale parameters). 
cpinteg_interp.m function: this function (Figure 90) numerically solves the PTS joint probability integral (Eq. 14). If the plot status is set on in this function, then a graphical display of the solution will also be displayed as previously shown in Figure 80.

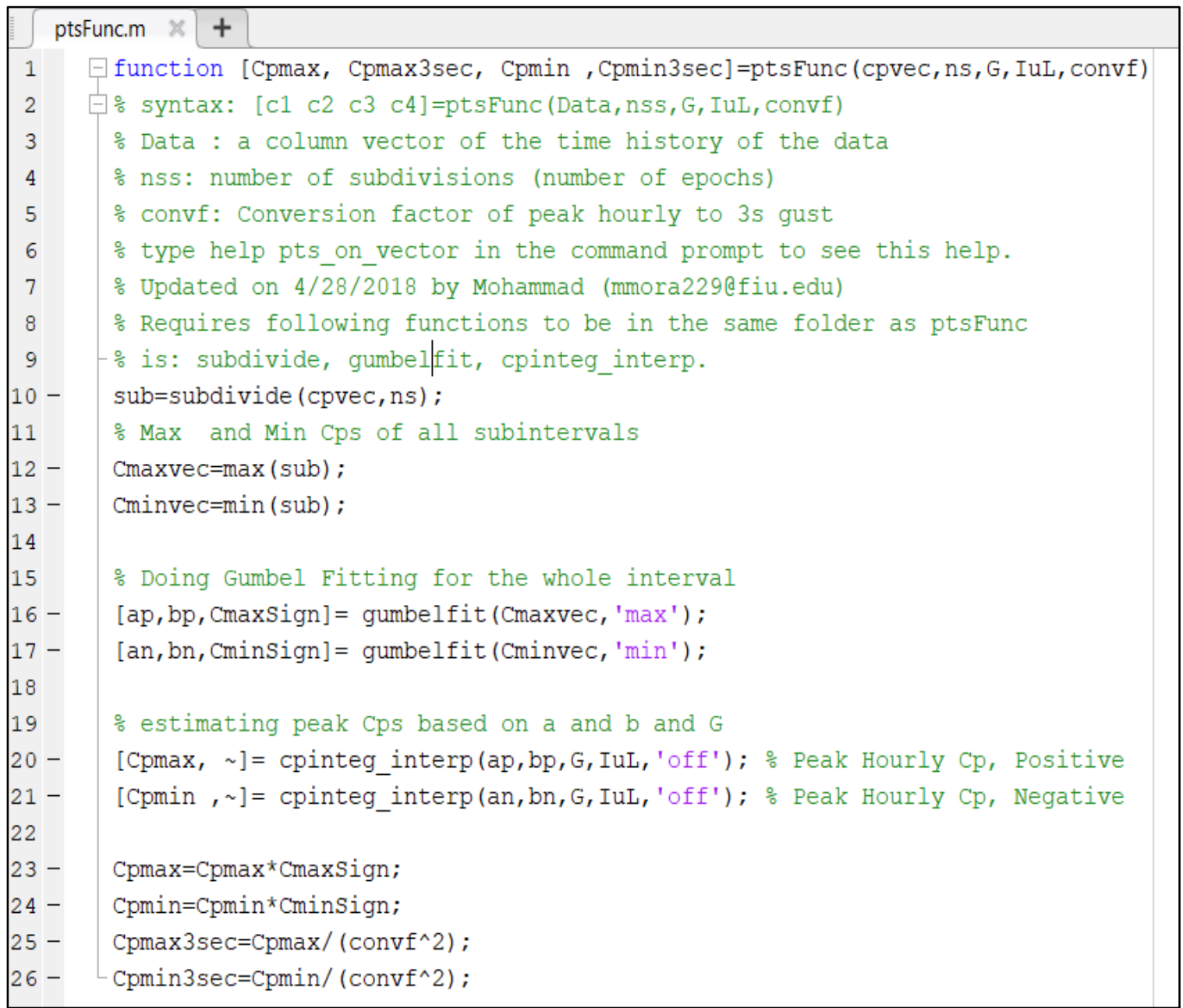

Figure 87 - MATLAB code for PTS analysis 


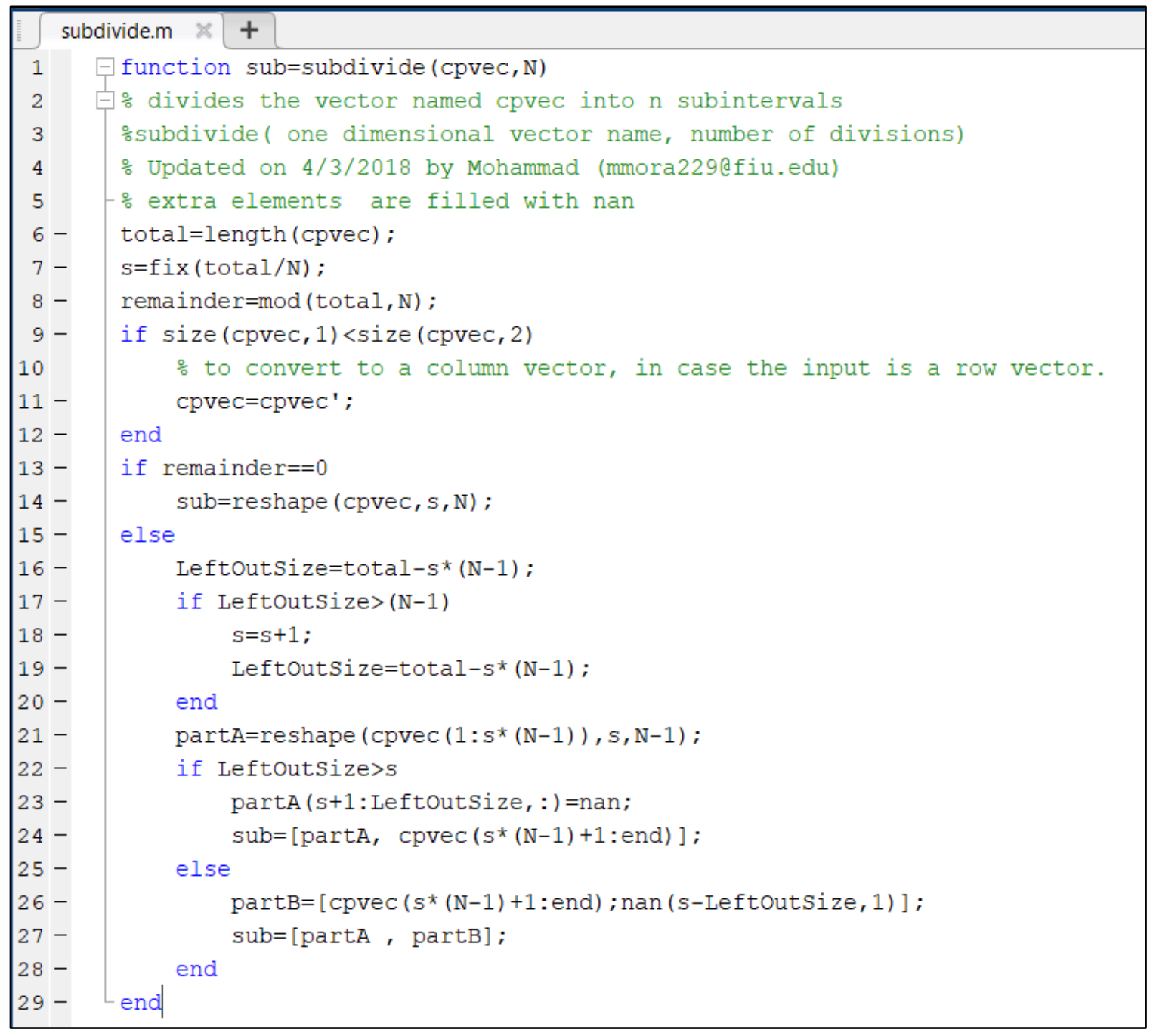

Figure 88 - MATLAB code for dividing time histories into subintervals 


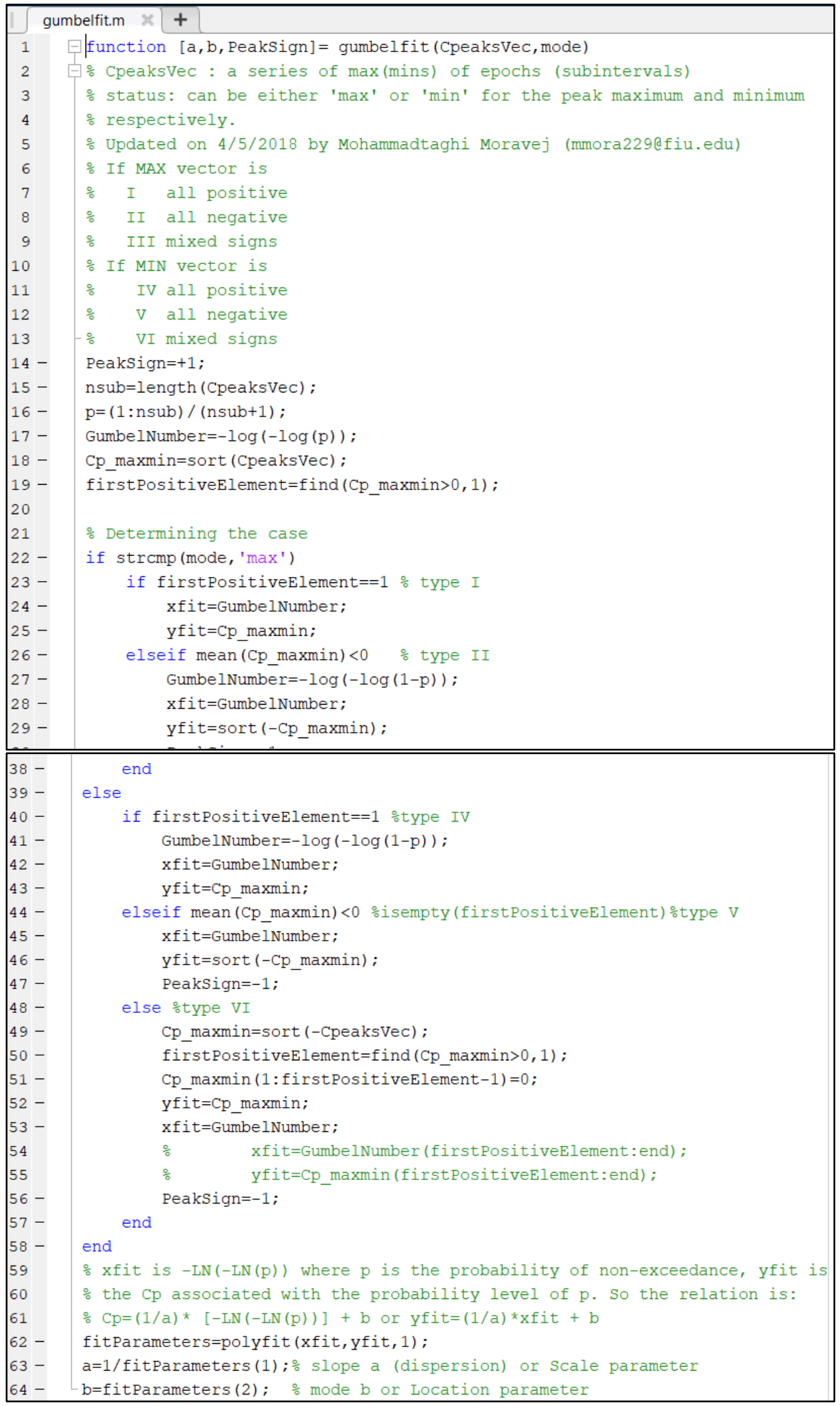

Figure 89 - MATLAB code for Gumbel fit 


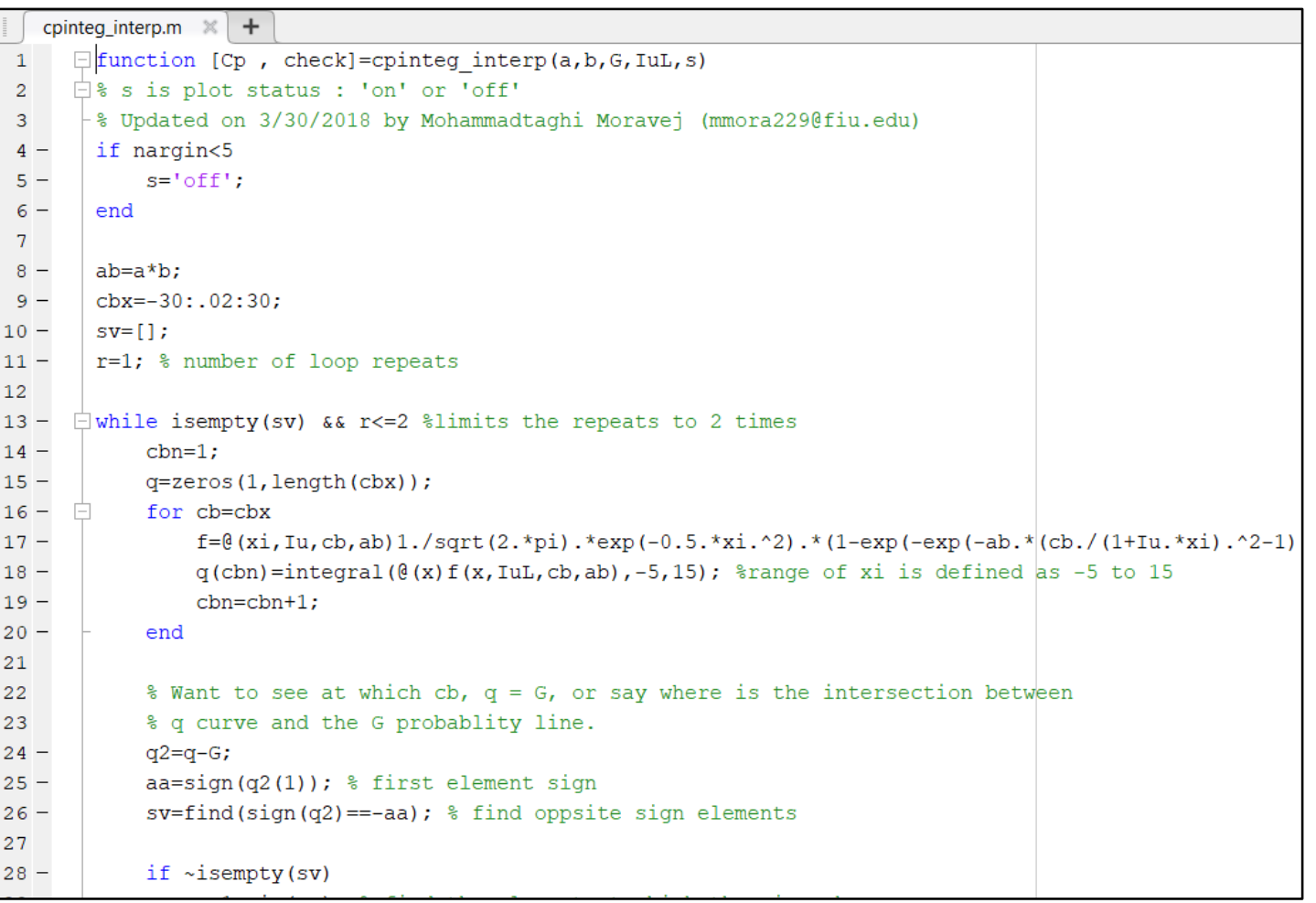

Figure 90 - MATLAB code for the numerical solution of the PTS integral 


\subsection{MATLAB Codes \& Procedure to Assess Flow Parameters}

\section{Wind Speed and Turbulence Profiles \& Spectra}

A MATLAB live code was developed (Figure 91) to plot the mean wind speed and turbulence intensity profiles against the corresponding full-scale values obtained from ESDU 85020[64]. The code generates plots normalized to the building eave height and the wind speed at the eave height. To calculate the ESDU values based on a given terrain condition and height, the "windESDU.m" code developed by Ashkan Rasouli was used. The live code was also extended to plot the nondimensionalized turbulence power spectra of the model and the full-scale (Figure 91,Figure 92). To smooth the spectra from Wall of Wind data, a blocking method was used by which the whole time history of the recorded wind speed is divided into smaller blocks of the size $2^{\mathrm{n}}$, and then the spectra obtained from all blocks are averaged to obtain a final smoothed spectrum. If the record length is long enough, then by choosing a higher value for $\mathrm{n}$, a smoother curve is attainable. The program used to smooth the spectra is called "spectrablocking.m" which is shown in Figure 93.

\section{Estimation of Turbulence Length Scale}

To estimate the integral length scale of the turbulence $\left({ }^{x} L_{u}\right)$, a program was developed as shown in Figure 94 . The estimation approach is based on a spectrum fit where the power spectrum of the wind speed is fitted to a Von Karman model through a least square regression to find the ${ }^{x} L_{u}$ that creates the best fit. Figure 95 shows the graphical output of the program where the scattered spectrum coordinates are fitted to a Von Karman spectrum. 


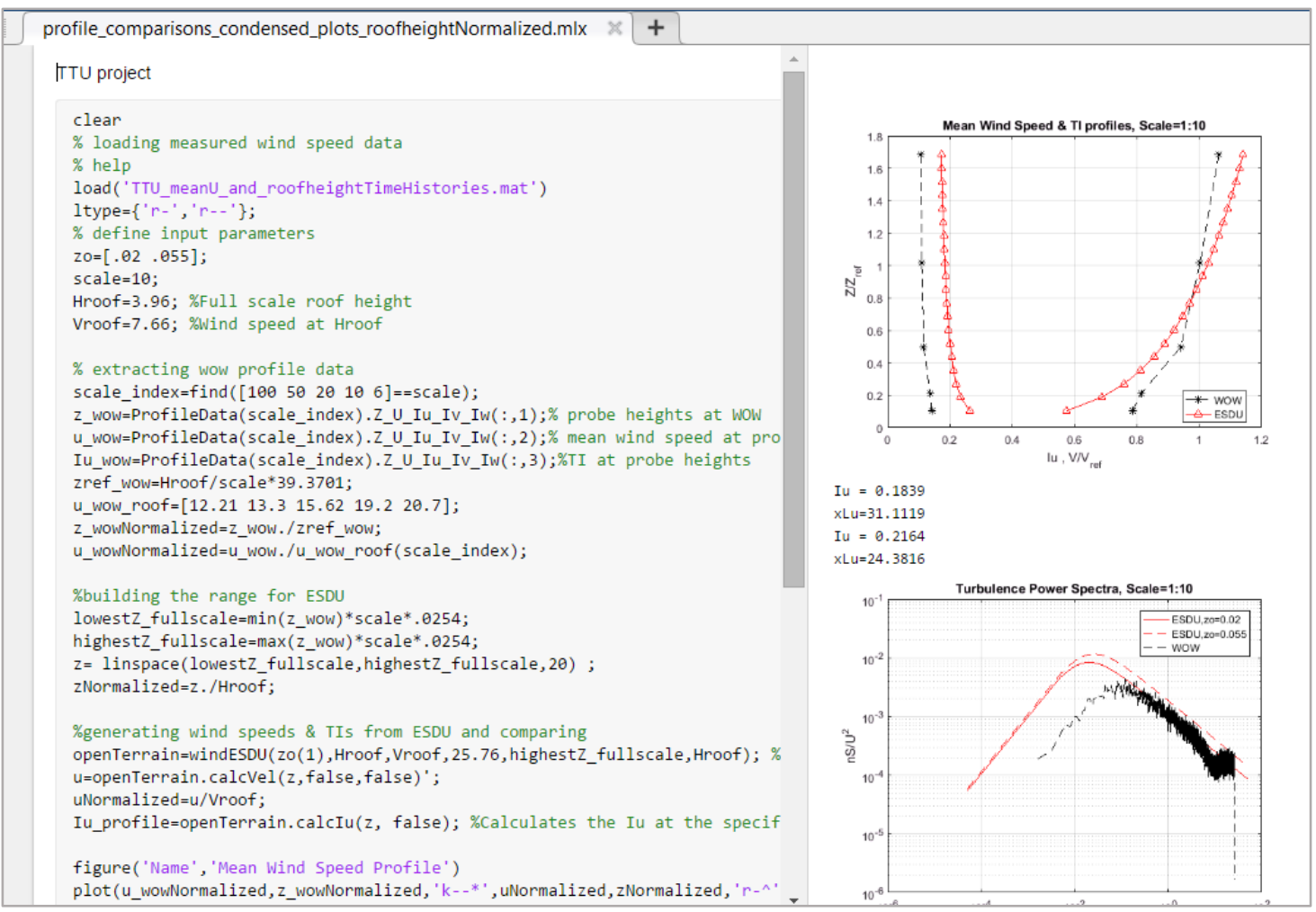

Figure 91 - Live MATLAB code to plot wind speed and turbulence spectra

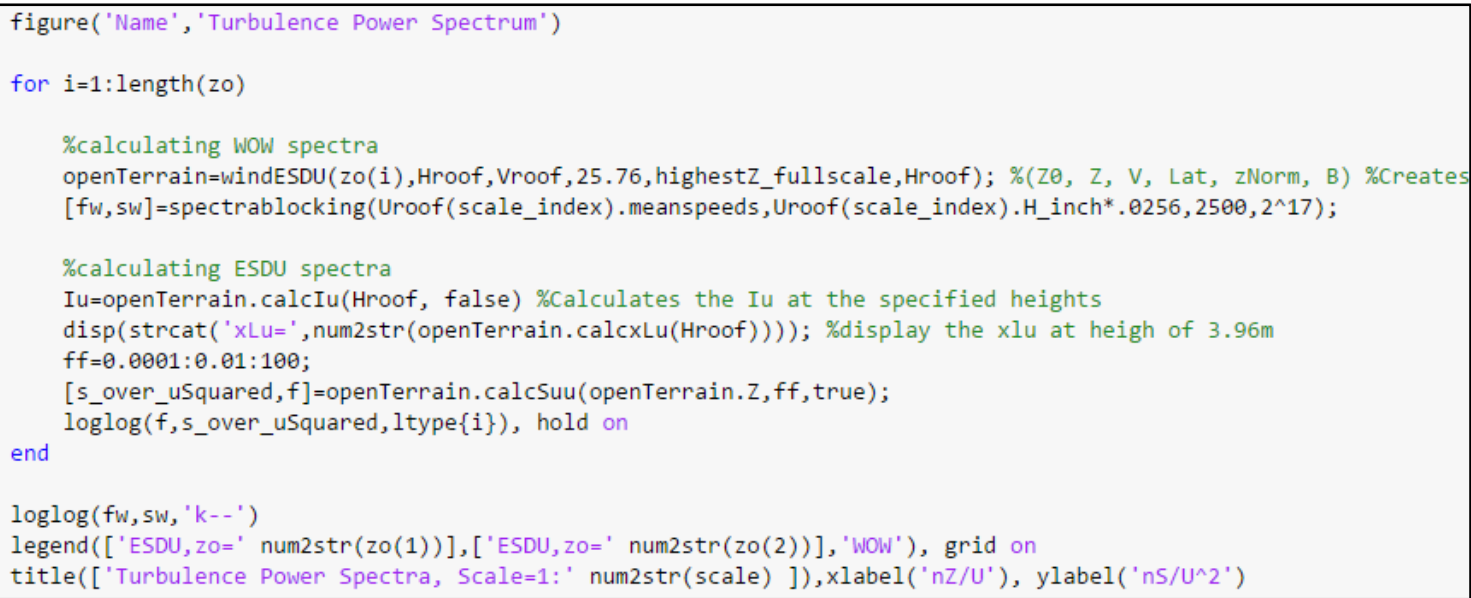

Figure 92 - MATLAB code to plot model and full-scale turbulence power spectra 


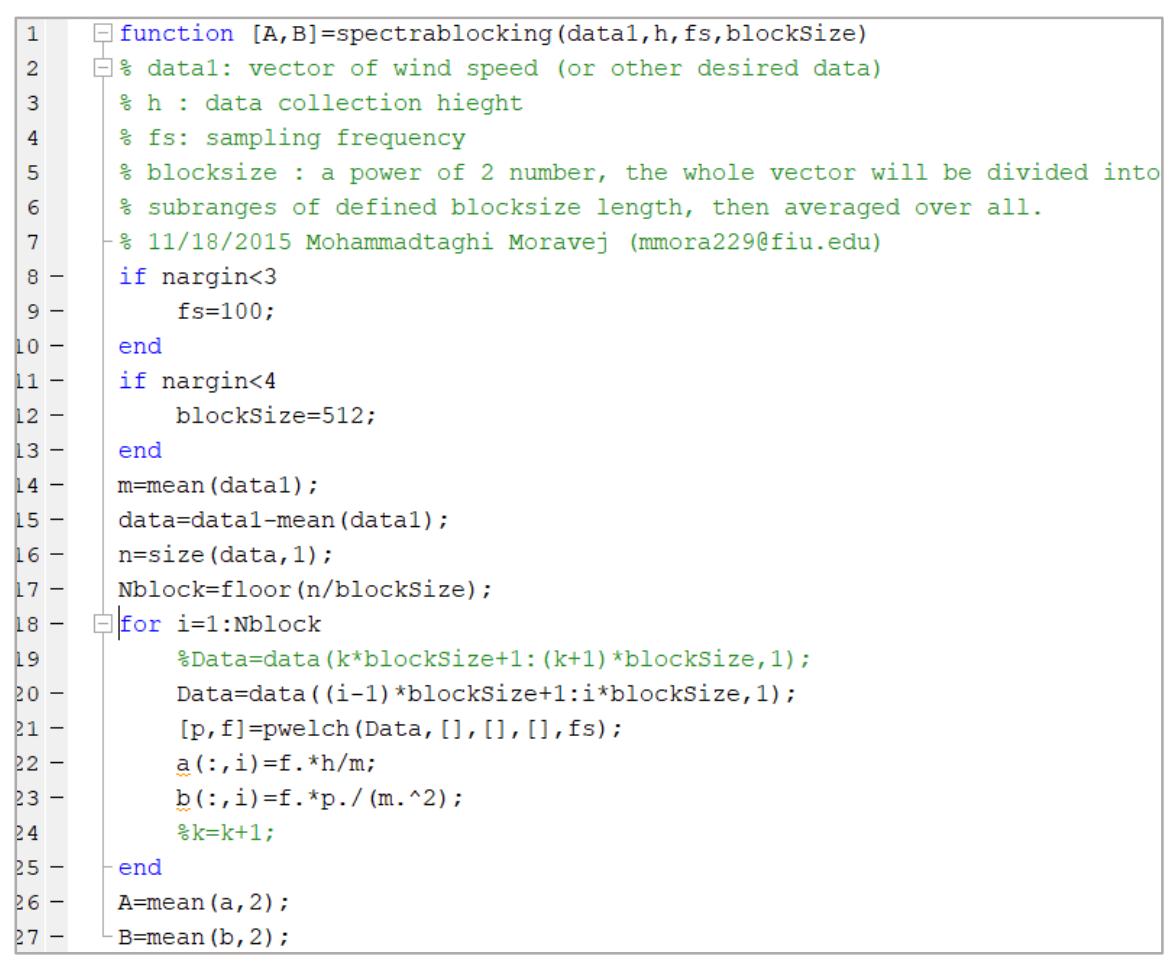

Figure 93 - Spectra smoothing code

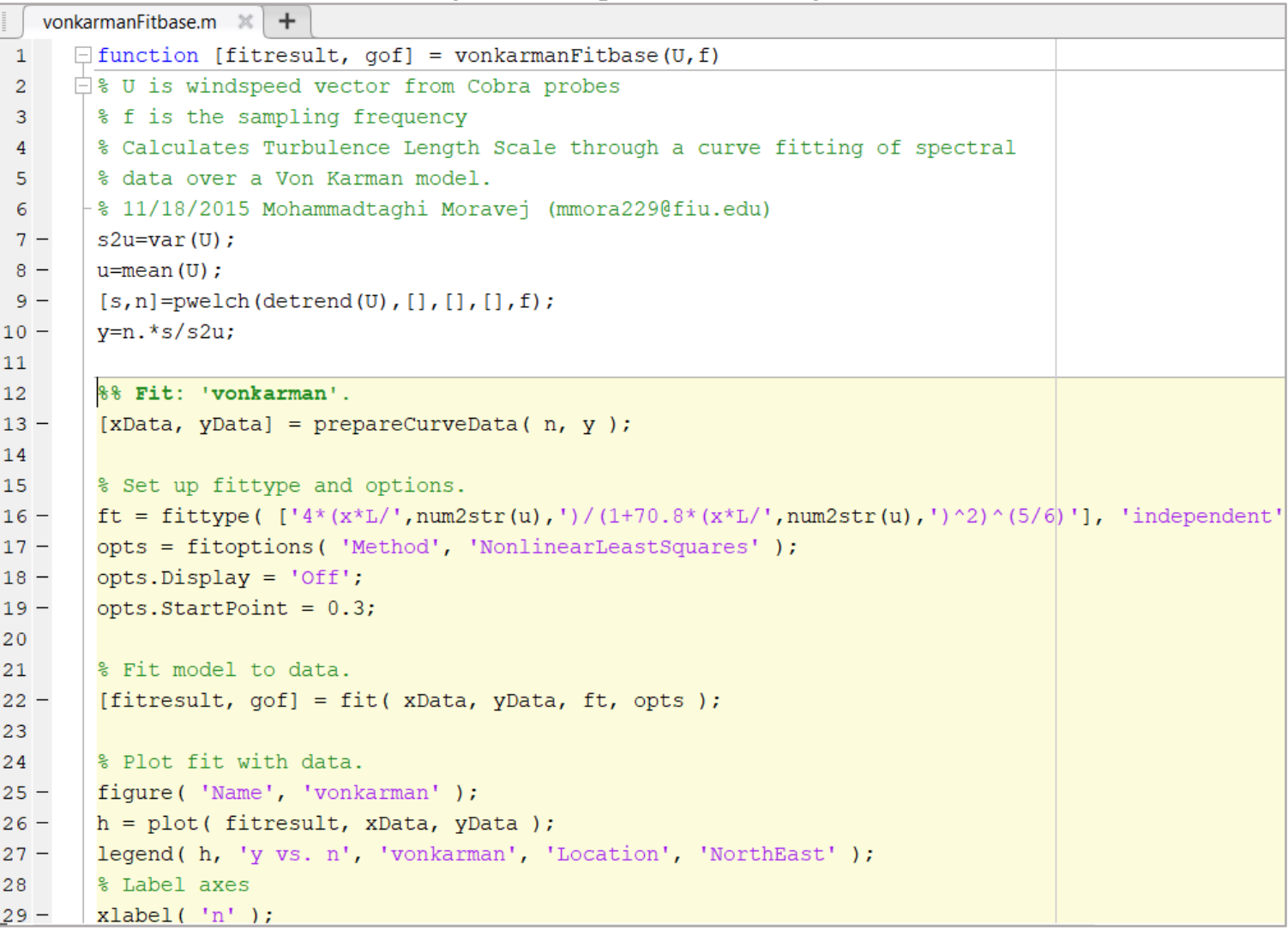

Figure 94 - MATLAB code for power spectra curve fitting to estimate ${ }^{x} L_{u}$ 


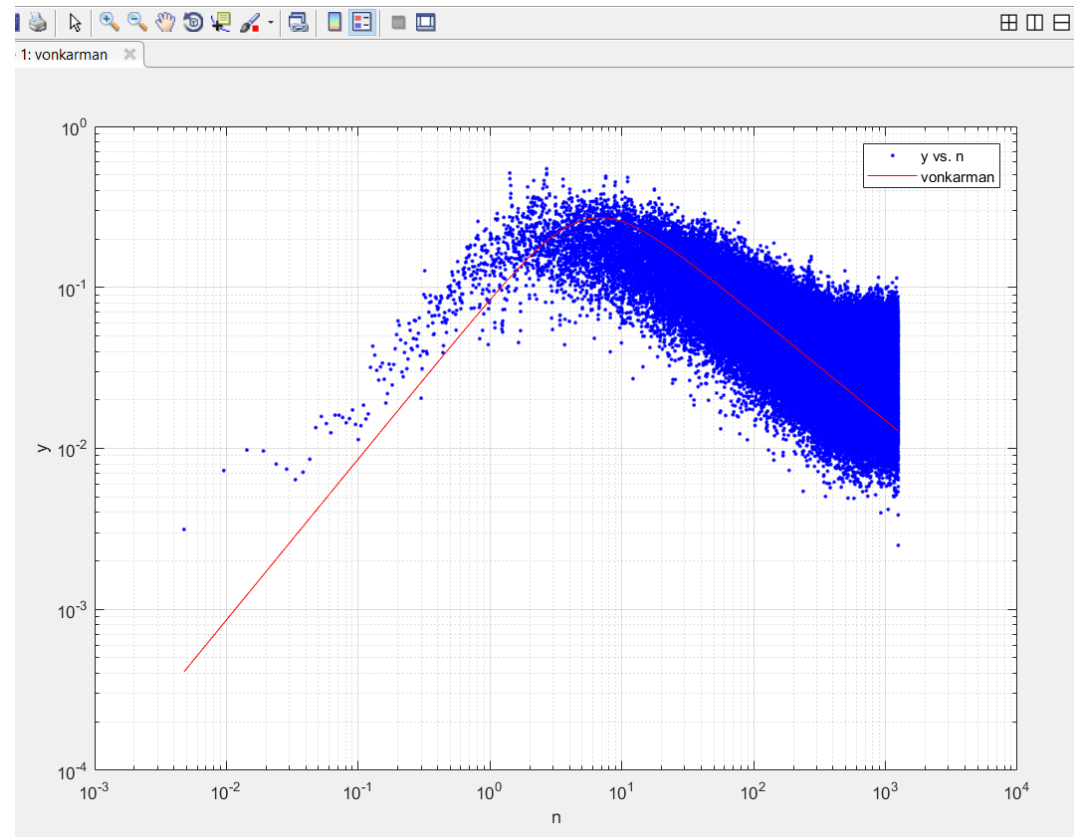

Figure 95 - Power spectra curve fitting to estimate ${ }^{x} L_{u}$ 


\subsection{MATLAB Codes and Procedure to Read TTU Field Data}

Two sets of TTU field data are available to use in the full-scale comparisons: One is a summary PDF file which includes the summary of the test statistics, flow conditions and the tap-wise plot of the mean, peak and rms $\mathrm{C}_{\mathrm{p}}$ results. Besides that, the CSV files which include the time history of all the collected data are also available. To avoid the errors in a manual look up of the data from the summary file, a MATLAB code was developed to automatically read the required data from the CSV files, and store them in a format readable by the rest of the codes in the TTU models data analysis tools. The default tap layout used throughout this study is as shown in Figure 96. As described in this figure, the definition of the wind directions is different between WOW models and the TTU which should be noticed during comparisons. A view of one of the CSV data files is shown in Figure 97. Each data file has 317 columns among which 204 columns show the results from the pressure taps. Those columns are scattered, and the coupling table presented in Figure 98 shows the relation between the column number in the data file and the actual tap number on the model. For instance, to find the time history of instantaneous pressure coefficients of tap\#1 (Figure 96), the data from the column 60 (Figure 98) of the CSV file should be read and analyzed. A MATLAB code, as shown in Figure 99, was designed for this purpose which reads all the pressure tap data, calculates the mean, maximum and minimum values of the pressure coefficients and stores them in MAT format. 


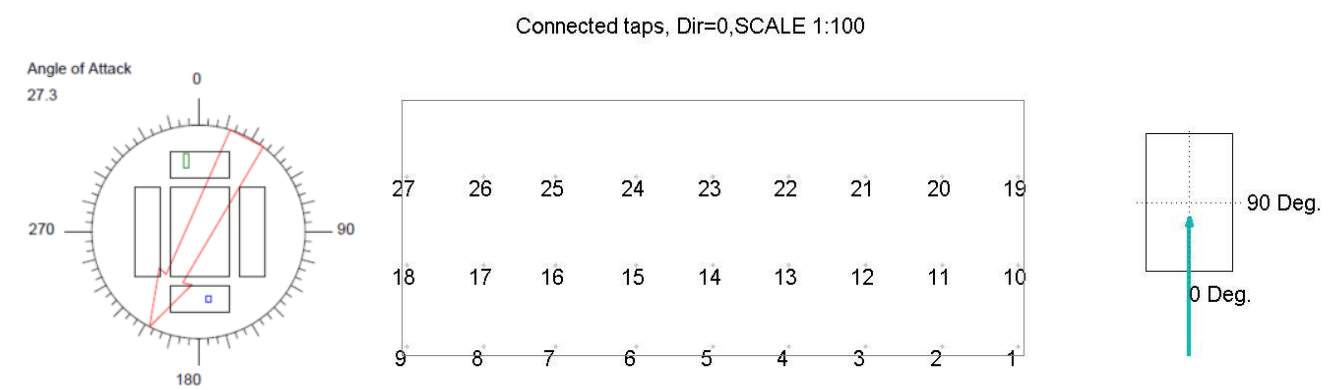

TTU directions definition

WOW directions definition

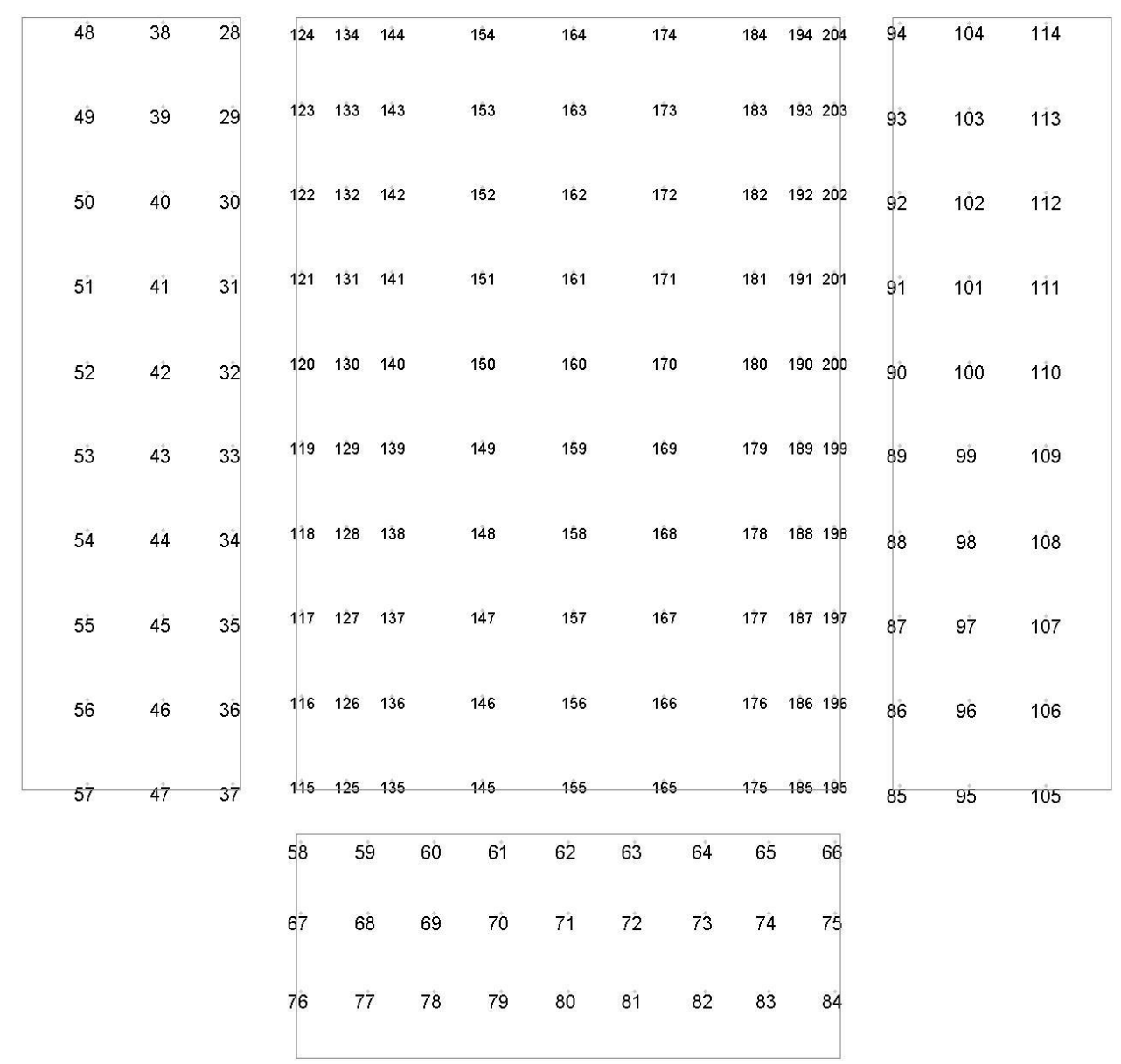

Figure 96 - Tap layout and numbering 


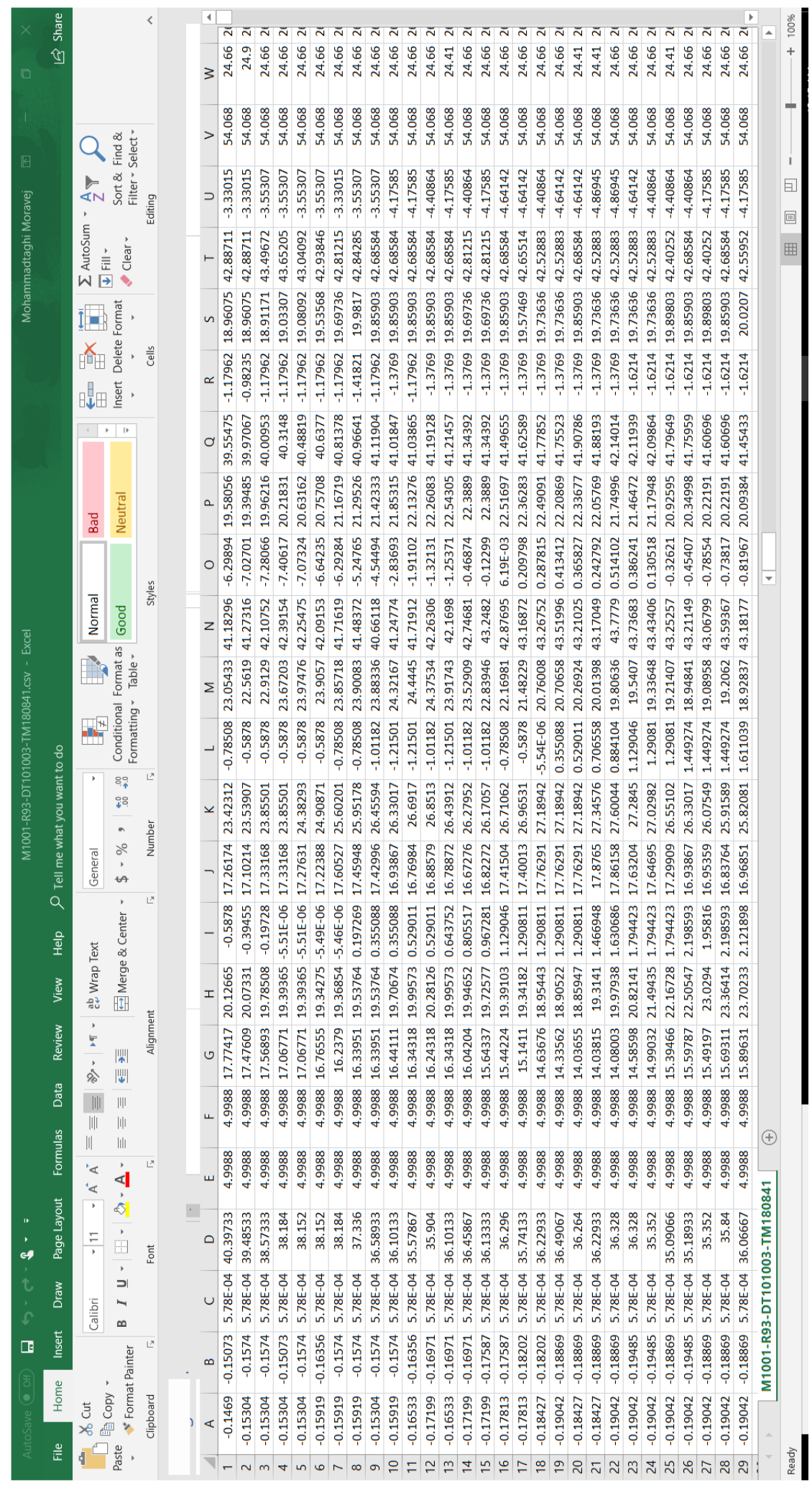

Figure 97 - A sample TTU data file 


\begin{tabular}{|c|c|c|c|c|c|c|c|c|c|c|c|}
\hline Tap No; & Order & TapName & Tap No; & Order & TapName & Tap No; & Order & TapName & Tap No; & Order & TapName \\
\hline 1 & 60 & B_09_G027 & 52 & 162 & H_34_G100 & 103 & 70 & C_11_G032 & 154 & 185 & I_39_G118 \\
\hline 2 & 56 & B_08_G023 & 53 & 154 & G_33_097 & 104 & 67 & C_10_G029 & 155 & 257 & K_58_G200 \\
\hline 3 & 54 & B_07_G021 & 54 & 151 & G_32_094 & 105 & 98 & D_19_G055 & 156 & 252 & K_56_G191 \\
\hline 4 & 51 & B_06_G018 & 55 & 148 & G_31_091 & 106 & 95 & D_18_G052 & 157 & 247 & K_54_G182 \\
\hline 5 & 48 & B_05_G015 & 56 & 145 & G_30_088 & 107 & 92 & D_17_G049 & 158 & 242 & K_52_G173 \\
\hline 6 & 41 & A_04_G012 & 57 & 142 & G_29_085 & 108 & 89 & D_16_G046 & 159 & 237 & K_50_G164 \\
\hline 7 & 38 & A_03_G009 & 58 & 136 & F_28_G084 & 109 & 86 & D_15_G043 & 160 & 227 & J_48_G155 \\
\hline 8 & 35 & A_02_G006 & 59 & 133 & F_27_G081 & 110 & 78 & C_14_G040 & 161 & 222 & J_46_G146 \\
\hline 9 & 32 & A_01_G003 & 60 & 130 & F_26_G078 & 111 & 75 & C_13_G037 & 162 & 217 & J_44_G137 \\
\hline 10 & 59 & B_09_G026 & 61 & 127 & F_25_G075 & 112 & 72 & C_12_G034 & 163 & 212 & J_42_G128 \\
\hline 11 & 57 & B_08_G024 & 62 & 124 & F_24_G072 & 113 & 69 & C_11_G031 & 164 & 207 & J_40_G119 \\
\hline 12 & 53 & B_07_G020 & 63 & 117 & E_23_G069 & 114 & 66 & C_10_G028 & 165 & 258 & K_58_G201 \\
\hline 13 & 50 & B_06_G017 & 64 & 114 & E_22_G066 & 115 & 283 & L_57_G196 & 166 & 253 & K_56_G192 \\
\hline 14 & 47 & B_05_G014 & 65 & 111 & E_21_G063 & 116 & 279 & L_55_G187 & 167 & 248 & K_54_G183 \\
\hline 15 & 40 & A_04_G011 & 66 & 108 & E_20_G060 & 117 & 275 & L_53_G178 & 168 & 243 & K_52_G174 \\
\hline 16 & 37 & A_03_G008 & 67 & 135 & F_28_G083 & 118 & 271 & L_51_G169 & 169 & 238 & K_50_G165 \\
\hline 17 & 34 & A_02_G005 & 68 & 132 & F_27_G080 & 119 & 267 & L_49_G160 & 170 & 228 & J_48_G156 \\
\hline 18 & 31 & A_01_G002 & 69 & 129 & F_26_G077 & 120 & 198 & I_47_G151 & 171 & 223 & J_46_G147 \\
\hline 19 & 58 & B_09_G025 & 70 & 126 & F_25_G074 & 121 & 194 & I_45_G142 & 172 & 218 & J_44_G138 \\
\hline 20 & 55 & B_08_G022 & 71 & 123 & F_24_G071 & 122 & 190 & I_43_G133 & 173 & 213 & J_42_G129 \\
\hline 21 & 52 & B_07_G019 & 72 & 116 & E_23_G068 & 123 & 186 & I_41_G124 & 174 & 208 & J_40_G120 \\
\hline 22 & 49 & B_06_G016 & 73 & 113 & E_22_G065 & 124 & 182 & I_39_G115 & 175 & 259 & K_58_G202 \\
\hline 23 & 46 & B_05_G013 & 74 & 110 & E_21_G062 & 125 & 284 & L_57_G197 & 176 & 254 & K_56_G193 \\
\hline 24 & 39 & A_04_G010 & 75 & 107 & E_20_G059 & 126 & 280 & L_55_G188 & 177 & 249 & K_54_G184 \\
\hline 25 & 36 & A_03_G007 & 76 & 134 & F_28_G082 & 127 & 276 & L_53_G179 & 178 & 244 & K_52_G175 \\
\hline 26 & 33 & A_02_G004 & 77 & 131 & F_27_G079 & 128 & 272 & L_51_G170 & 179 & 239 & K_50_G166 \\
\hline 27 & 30 & A_01_G001 & 78 & 128 & F_26_G076 & 129 & 268 & L_49_G161 & 180 & 229 & J_48_G157 \\
\hline 28 & 176 & H_38_G114 & 79 & 125 & F_25_G073 & 130 & 199 & I_47_G152 & 181 & 224 & J_46_G148 \\
\hline 29 & 173 & H_37_G111 & 80 & 122 & F_24_G070 & 131 & 195 & I_45_G143 & 182 & 219 & J_44_G139 \\
\hline 30 & 170 & H_36_G108 & 81 & 115 & E_23_G067 & 132 & 191 & I_43_G134 & 183 & 214 & J_42_G130 \\
\hline 31 & 167 & H_35_G105 & 82 & 112 & E_22_G064 & 133 & 187 & I_41_G125 & 184 & 209 & J_40_G121 \\
\hline 32 & 164 & H_34_G102 & 83 & 109 & E_21_G061 & 134 & 183 & I_39_G116 & 185 & 260 & K_58_G203 \\
\hline 33 & 156 & G_33_099 & 84 & 106 & E_20_G058 & 135 & 285 & L_57_G198 & 186 & 255 & K_56_G194 \\
\hline 34 & 153 & G_32_096 & 85 & 100 & D_19_G057 & 136 & 281 & L_55_G189 & 187 & 250 & K_54_G185 \\
\hline 35 & 150 & G_31_093 & 86 & 97 & D_18_G054 & 137 & 277 & L_53_G180 & 188 & 245 & K_52_G176 \\
\hline 36 & 147 & G_30_090 & 87 & 94 & D_17_G051 & 138 & 273 & L_51_G171 & 189 & 240 & K_50_G167 \\
\hline 37 & 144 & G_29_087 & 88 & 91 & D_16_G048 & 139 & 269 & L_49_G162 & 190 & 230 & J_48_G158 \\
\hline 38 & 175 & H_38_G113 & 89 & 88 & D_15_G045 & 140 & 200 & I_47_G153 & 191 & 225 & J_46_G149 \\
\hline 39 & 172 & H_37_G110 & 90 & 80 & C_14_G042 & 141 & 196 & I_45_G144 & 192 & 220 & J_44_G140 \\
\hline 40 & 169 & H_36_G107 & 91 & 77 & C_13_G039 & 142 & 192 & I_43_G135 & 193 & 215 & J_42_G131 \\
\hline 41 & 166 & H_35_G104 & 92 & 74 & C_12_G036 & 143 & 188 & I_41_G126 & 194 & 210 & J_40_G122 \\
\hline 42 & 163 & H_34_G101 & 93 & 71 & C_11_G033 & 144 & 184 & I_39_G117 & 195 & 261 & K_58_G204 \\
\hline 43 & 155 & G_33_098 & 94 & 68 & C_10_G030 & 145 & 286 & L_57_G199 & 196 & 256 & K_56_G195 \\
\hline 44 & 152 & G_32_095 & 95 & 99 & D_19_G056 & 146 & 282 & L_55_G190 & 197 & 251 & K_54_G186 \\
\hline 45 & 149 & G_31_092 & 96 & 96 & D_18_G053 & 147 & 278 & L_53_G181 & 198 & 246 & K_52_G177 \\
\hline 46 & 146 & G_30_089 & 97 & 93 & D_17_G050 & 148 & 274 & L_51_G172 & 199 & 241 & K_50_G168 \\
\hline 47 & 143 & G_29_086 & 98 & 90 & D_16_G047 & 149 & 270 & L_49_G163 & 200 & 231 & J_48_G159 \\
\hline 48 & 174 & H_38_G112 & 99 & 87 & D_15_G044 & 150 & 201 & I_47_G154 & 201 & 226 & J_46_G150 \\
\hline 49 & 171 & H_37_G109 & 100 & 79 & C_14_G041 & 151 & 197 & I_45_G145 & 202 & 221 & J_44_G141 \\
\hline 50 & 168 & H_36_G106 & 101 & 76 & C_13_G038 & 152 & 193 & I_43_G136 & 203 & 216 & J_42_G132 \\
\hline 51 & 165 & H_35_G103 & 102 & 73 & C_12_G035 & 153 & 189 & I_41_G127 & 204 & 211 & J_40_G123 \\
\hline
\end{tabular}

Figure 98 - Tap orders vs. the corresponding column numbers in the data file 


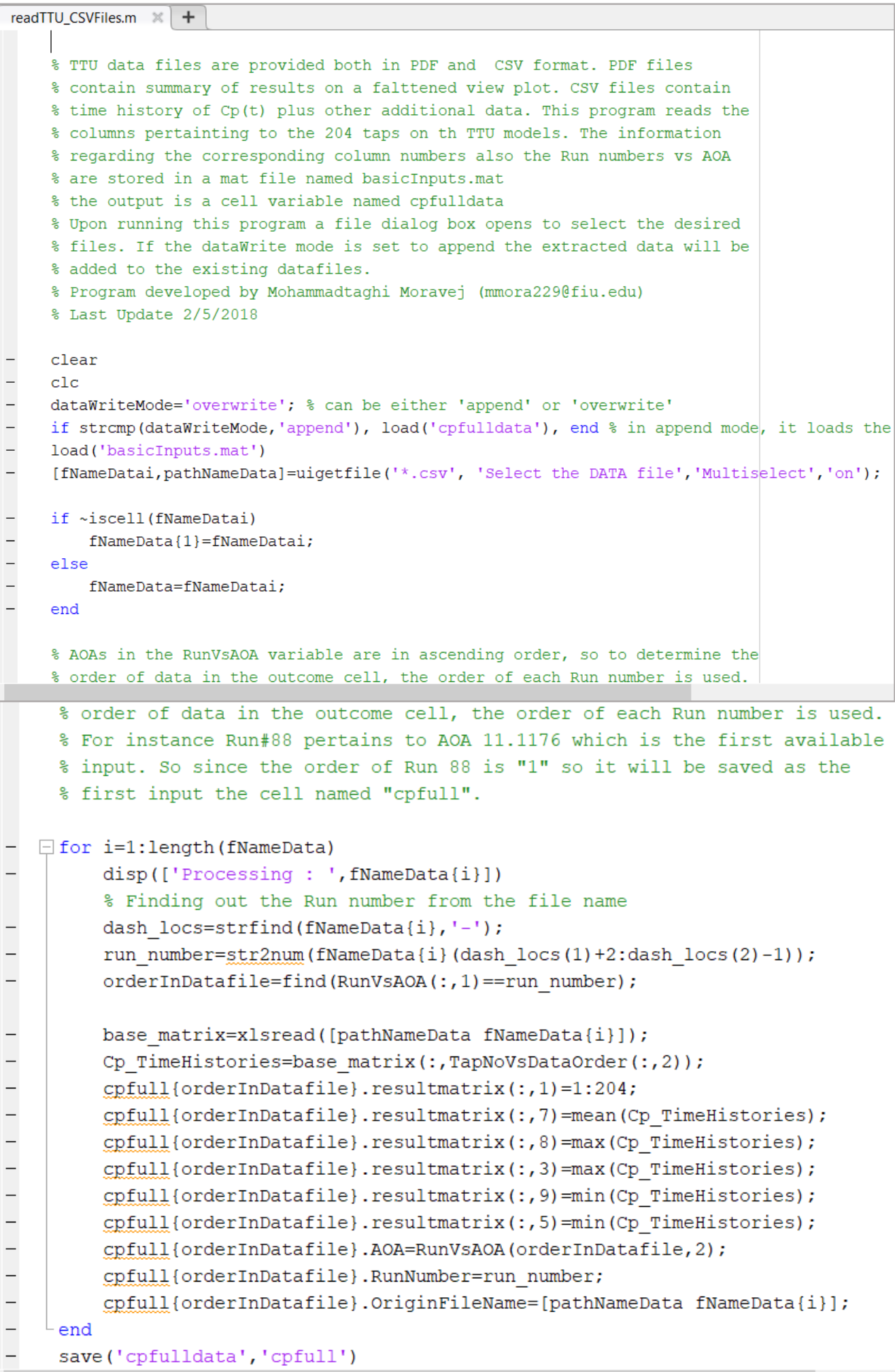

Figure 99 - MATLAB code to read TTU data files 


\subsection{Drawings \& Model Sketches}
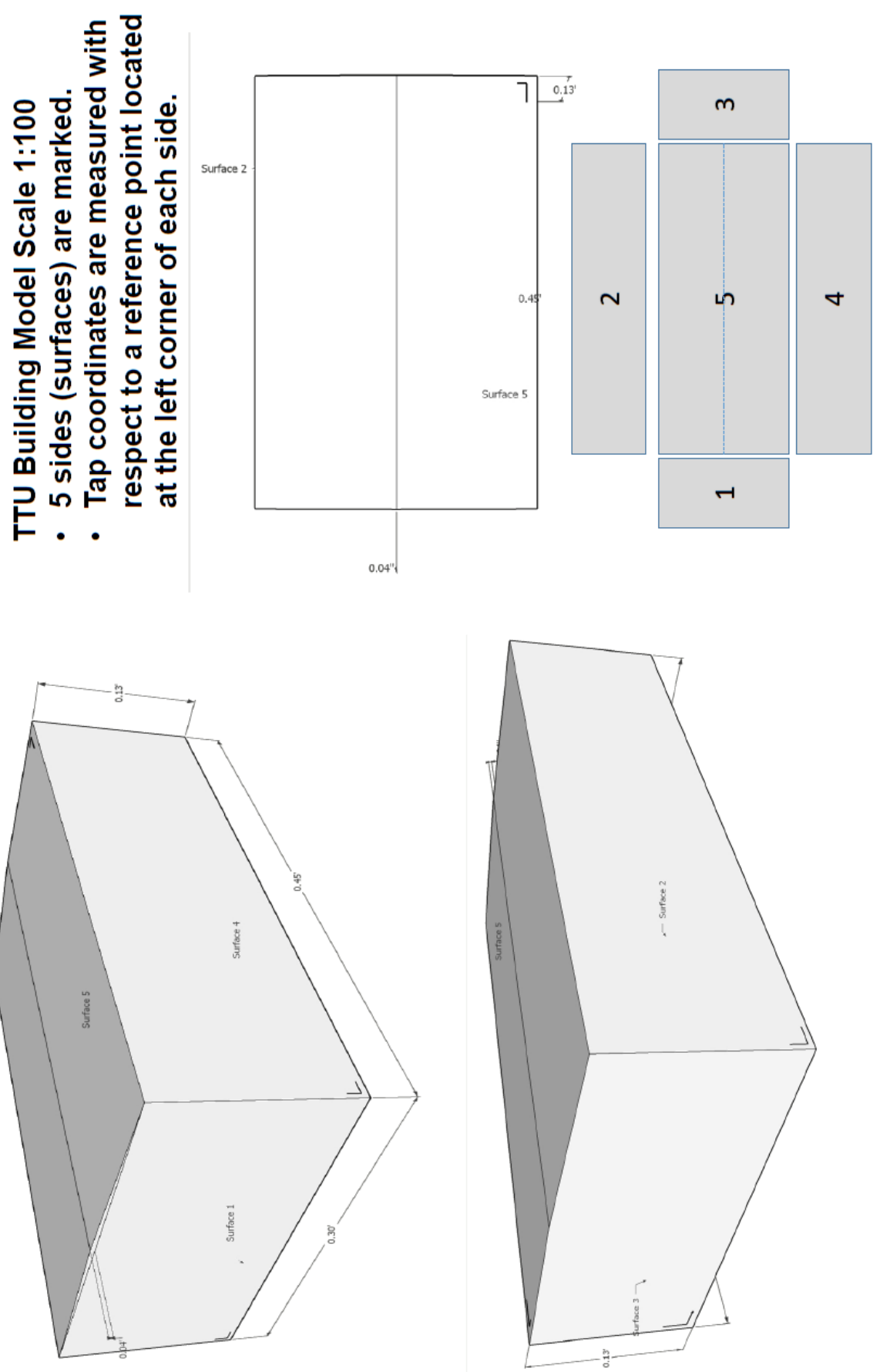

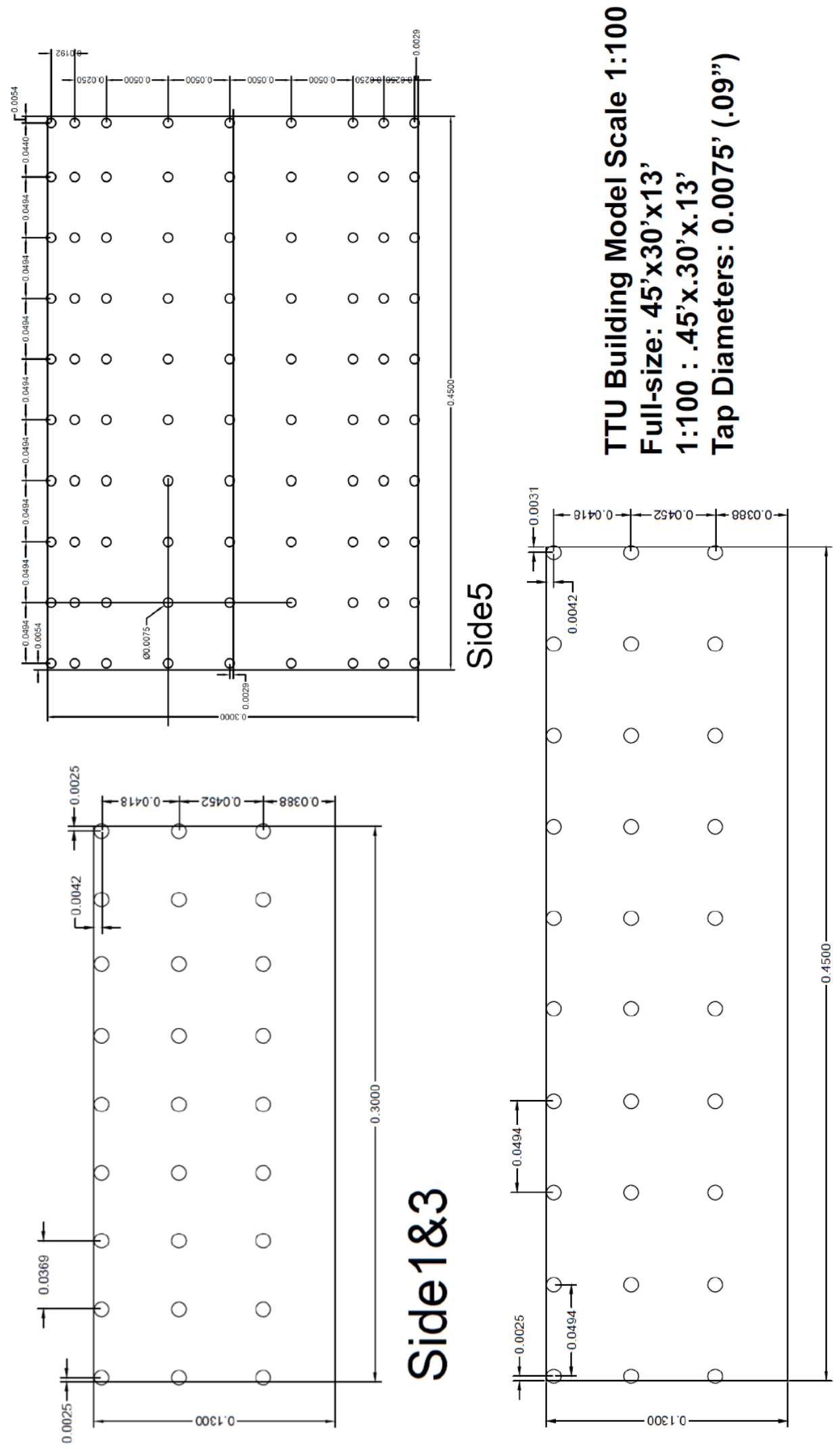

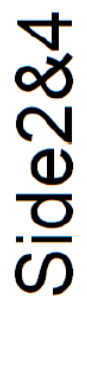




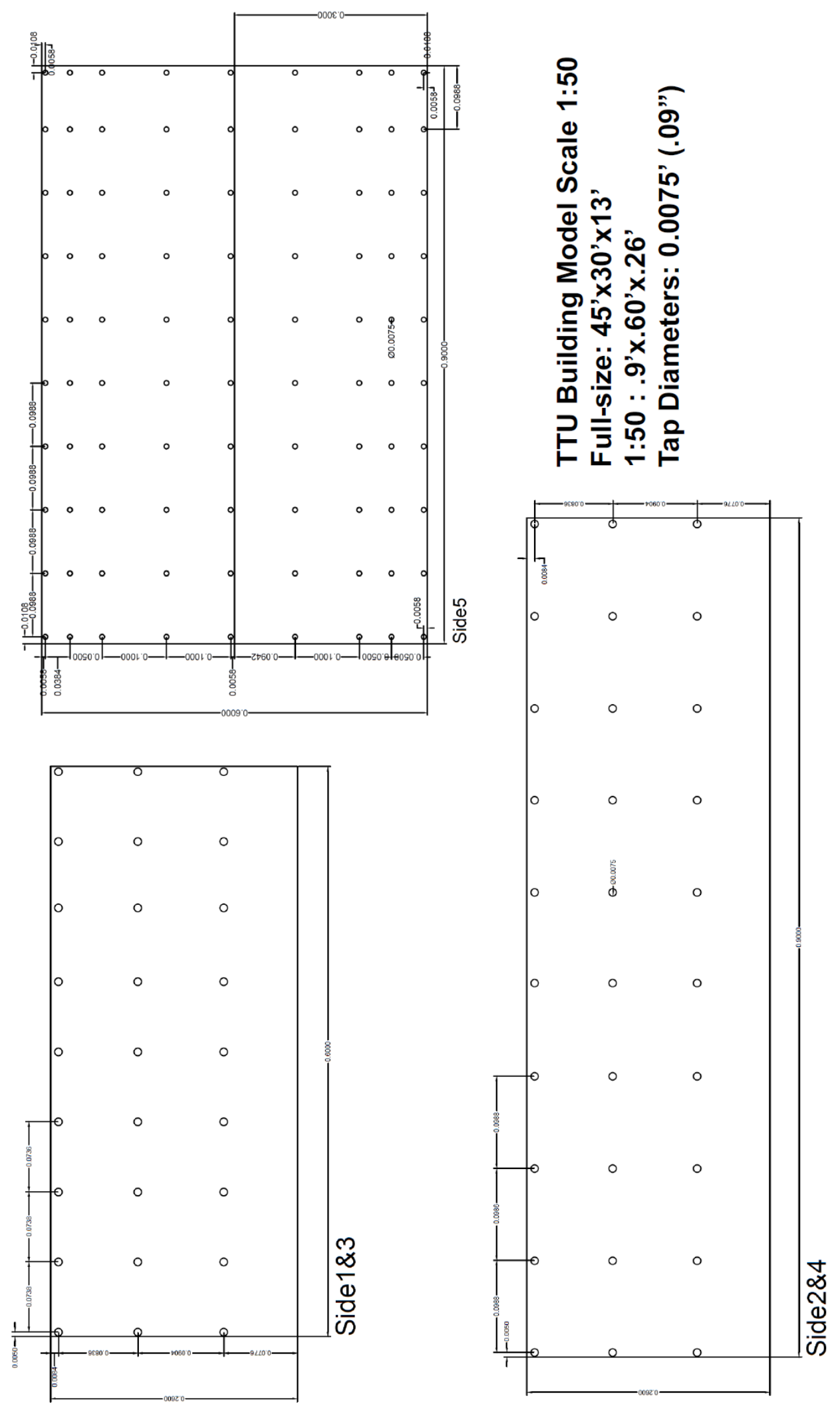



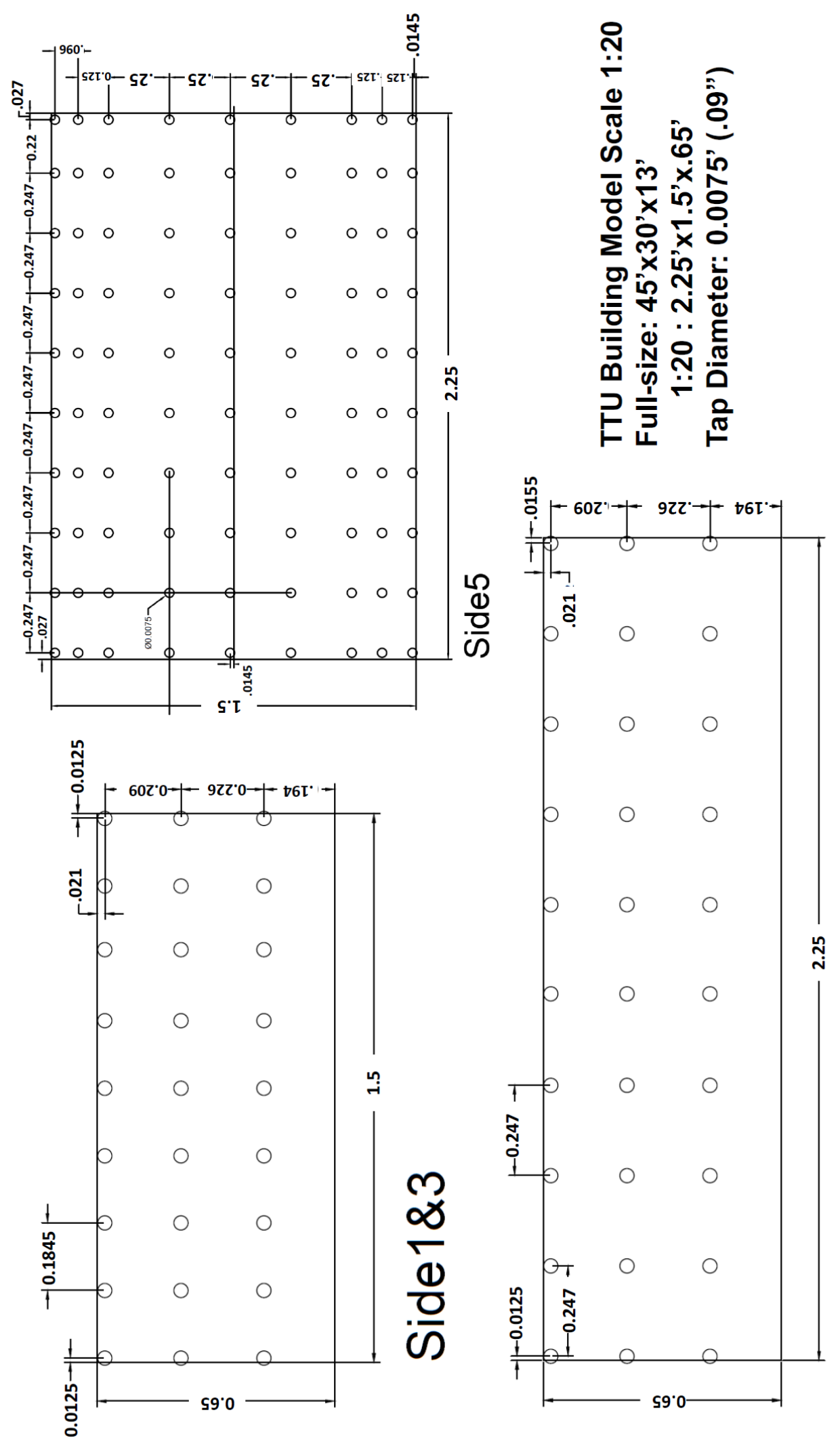

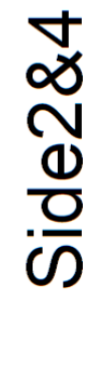




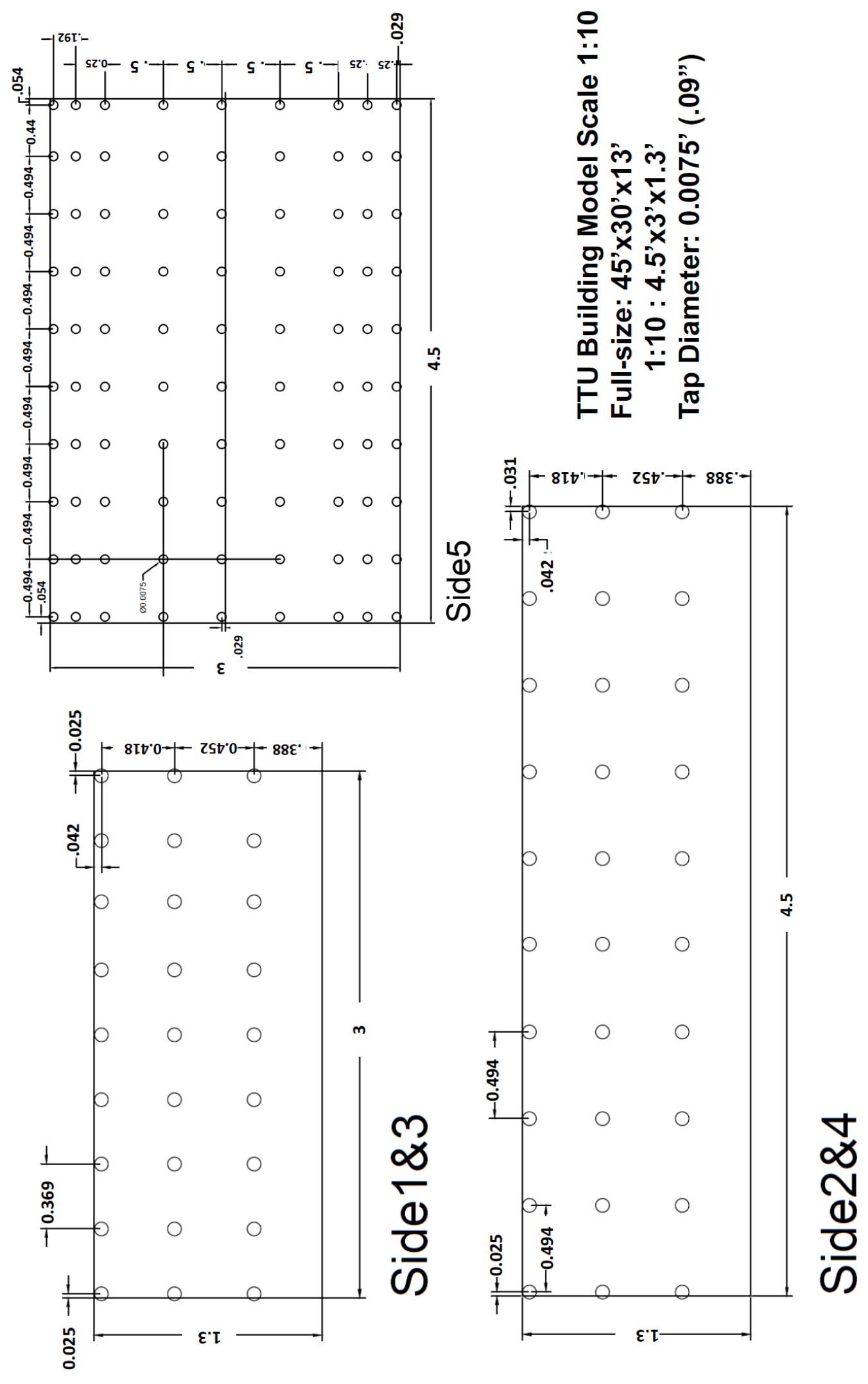




\subsection{Tubing Correction \& Filtering}

Generally based on the model sizes, various tube lengths are used to connect the model pressure taps to the pressure scanning system. It's known that a long tube introduces some distortions in the signal (i.e., pressure time history being collected) and the extent of the distortion depends on the length of the tube. So it is essential to correct the collected signal for the tubing distortions as the first step in the data analysis procedure. The method used at the Wall of Wind is based on the approach proposed by Irwin [57] and is briefly discussed here. The detailed information about the experimental setup and the analytical toolbox can be found in WOW-EF docs. The adopted procedure is as follows:

1- By using a signal generator, Scanivalve pressure sensors, amplifier-speaker set and data-acquisition hardware and software, two sets of signals were generated: a short tube signal where the sensor was directly connected to the signal output port with a short connector, and a long tube data by using the desired tube length (including the tubulations) to connect the signal generator to the sensor. The data were sampled at $2500 \mathrm{~Hz}$ and for a duration of 10 minutes. Figure 100 shows a schematic of the whole system.

2- A MATLAB program was developed (Figure 101) to estimate the transfer function between the two signals. The calculated transfer function for a tube of $3 \mathrm{ft}$ length is displayed in Figure 102a. This transfer function is comparable to the one calculated by Irwin [57] and is shown in Figure 102b.

3- After obtaining the transfer function, it was applied to the long tube data to correct for the distortions and to check whether the short tube data could be retrieved. Figure 
103 illustrates this comparison where the corrected signal is closely matching the short tube (intact) signal and indicates a significant improvement of the long tube (distorted) signal. A numerical comparison showed that the RMS of the differences between the long tube and short tube data was 0.0035 which was drastically dropped to a value of 0.0002 when the short tube signal and the corrected one were compared.

4- The above steps were repeated for all the tube lengths used in the 5 TTU models. Then the transfer function was applied to the pressure data to obtain the corrected time series of the pressures. All the data were filtered at $250 \mathrm{~Hz}$ (near half of the pressure data sampling rate) before application of the transfer function. Also, it is worthwhile to mention that the tube is expected to affect the fluctuating part of the collected signal so the mean values should be similar and unaffected by the tube. This was used as another verification tool where the mean pressures of the corrected signal were compared to those from the collected data (long tube data). 


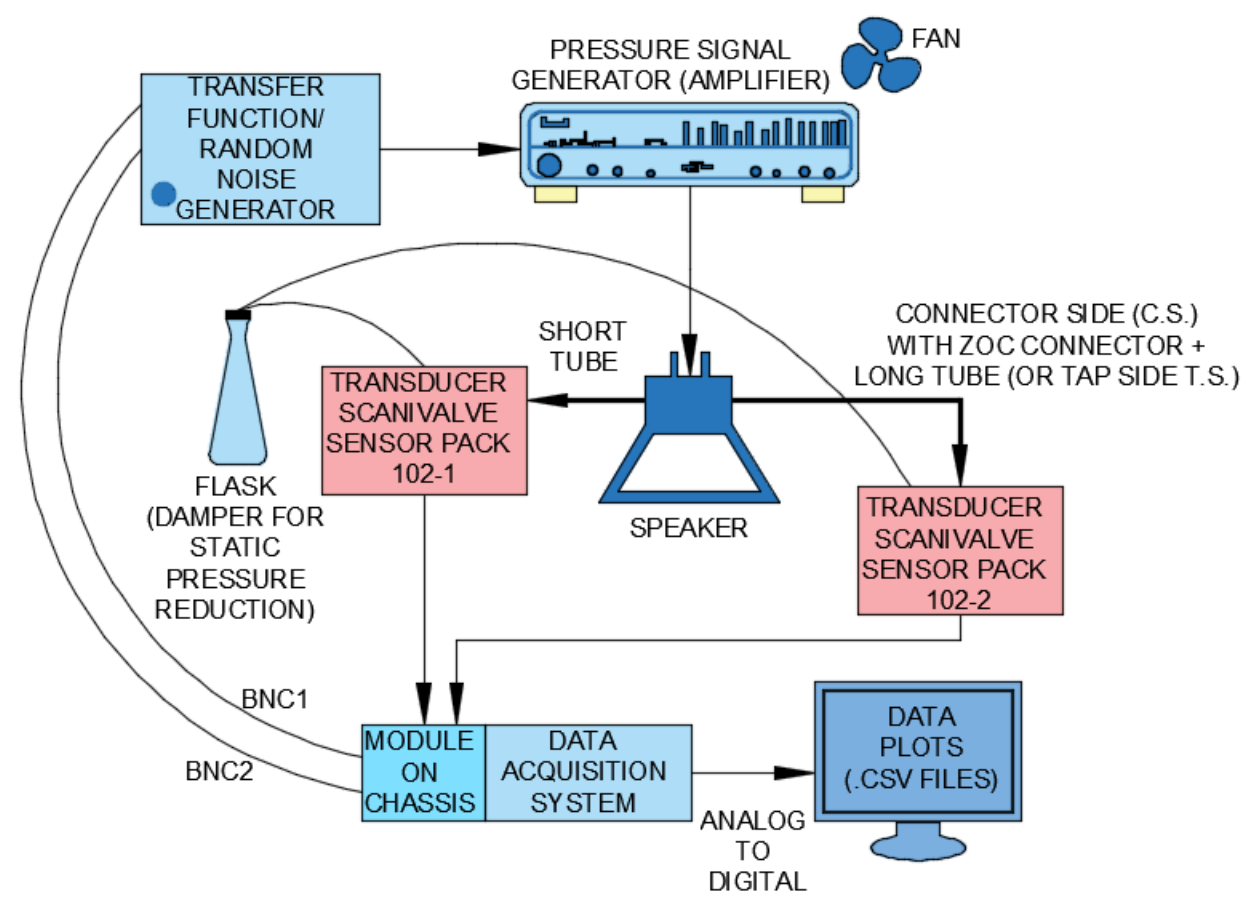

Figure 100 - Transfer function setup block diagram

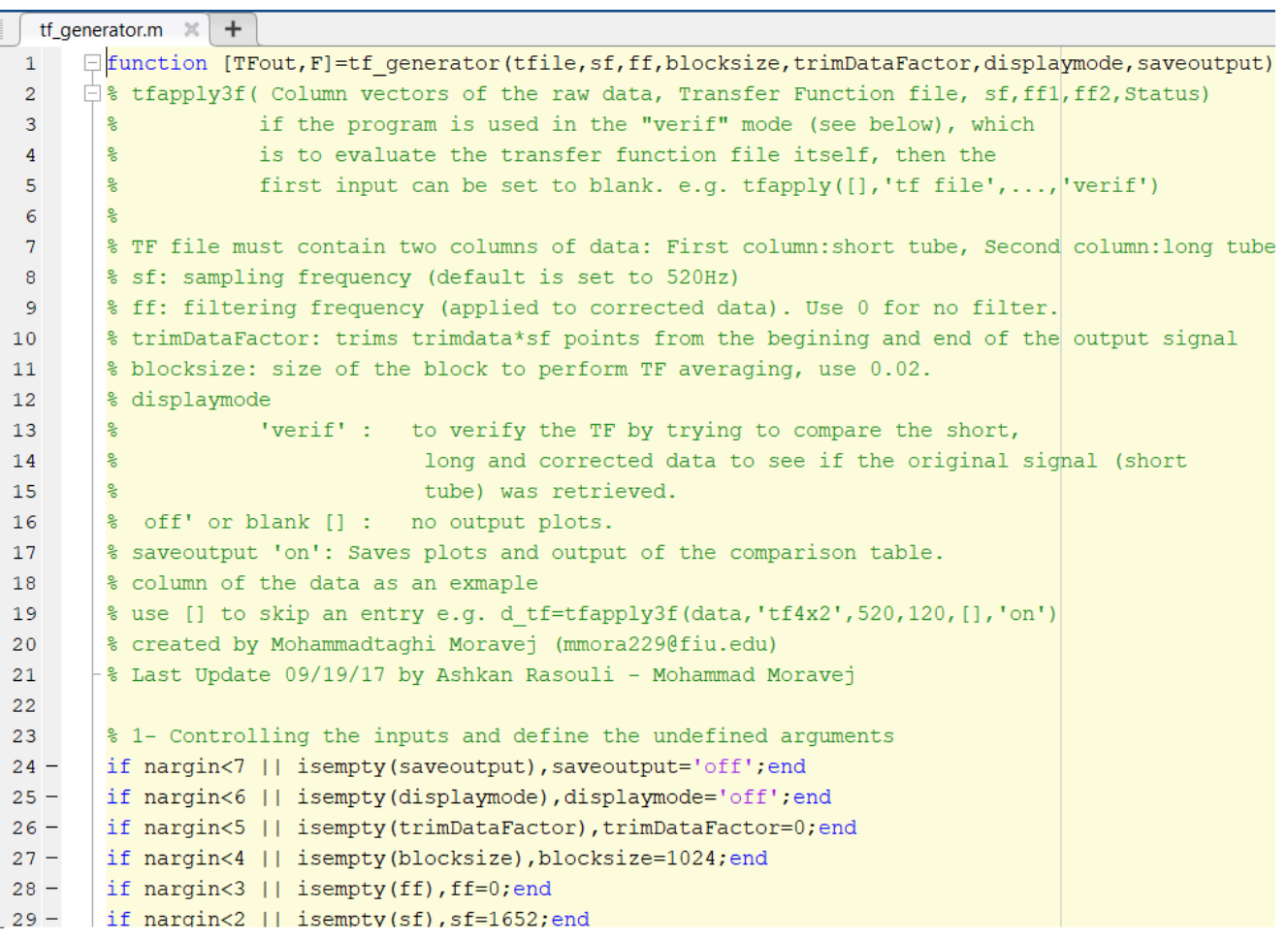

Figure 101 - MATLAB program to generate a transfer function from short and long tube data 

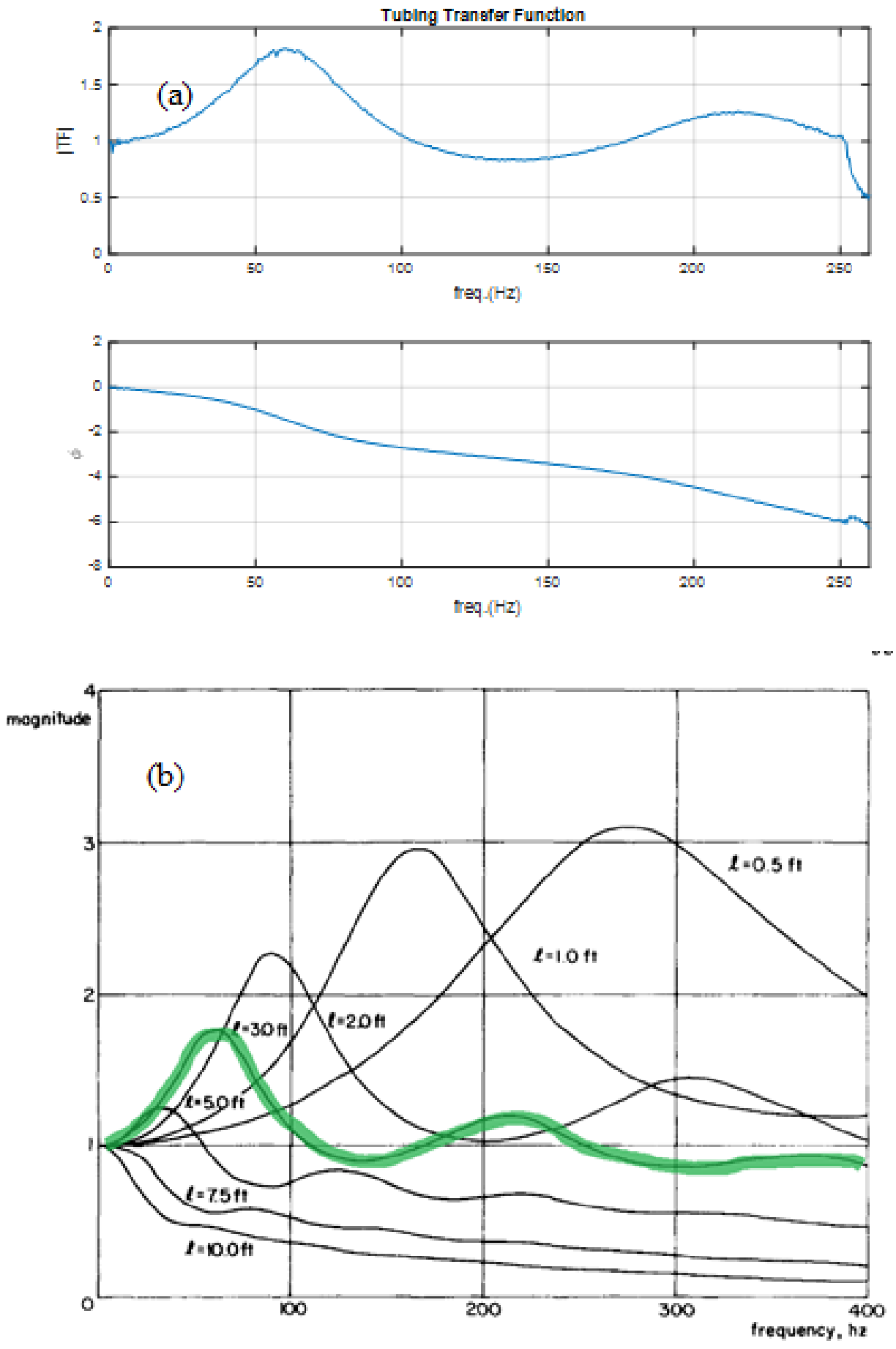

Figure 102 - Transfer function used in WOW (a) vs. TF calculated by Irwin [57] (b) 

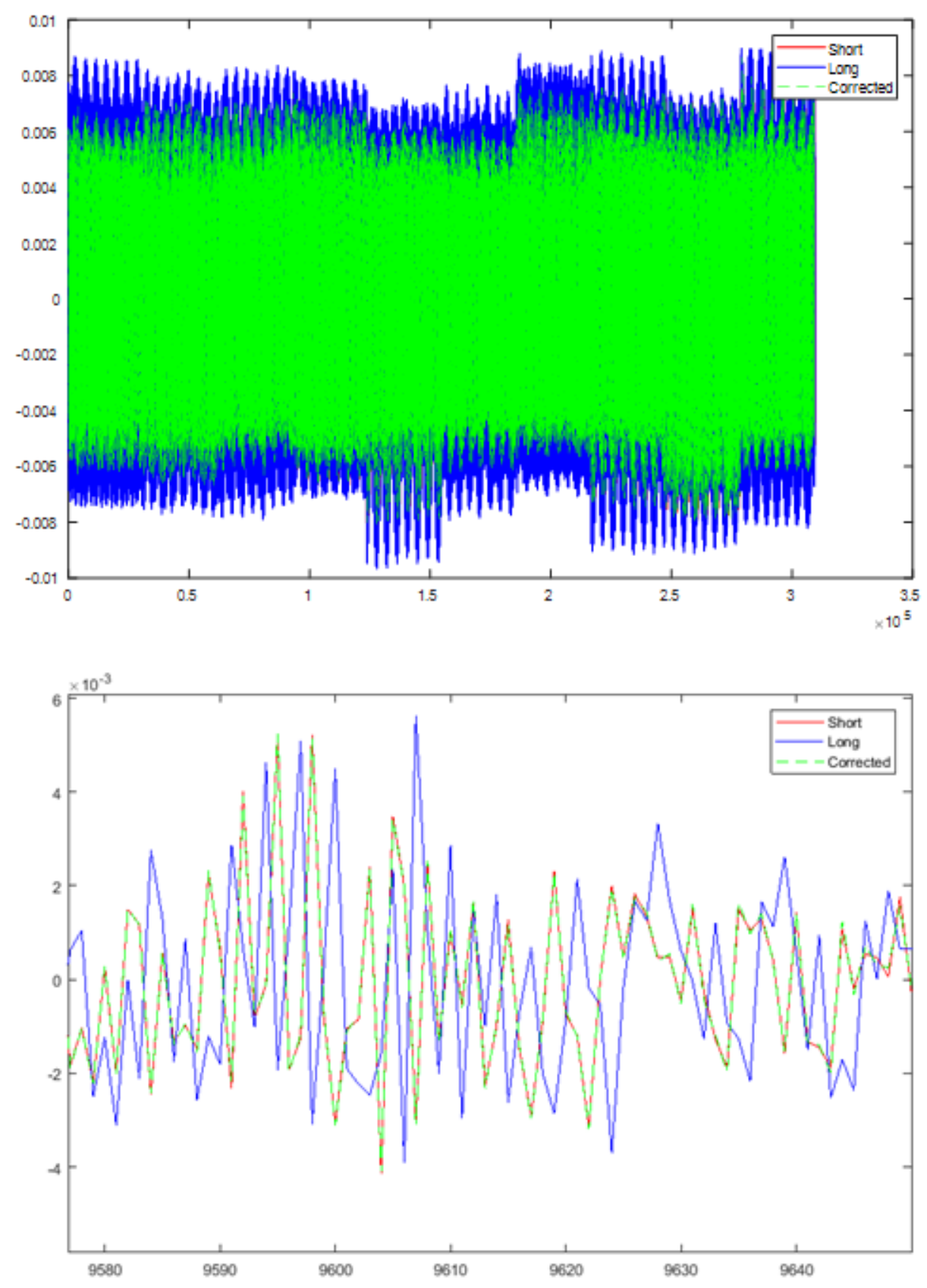

Figure 103 - Corrected signal versus the long tube and short tube signals 


\subsection{Suggested PTS Application Procedure for Taller Buildings with an Example}

Tall building models in wind tunnels are usually of very small scales, typically in the 1:200 to 1:400 range. These scales are proper enough to study the overall aerodynamic response of the structure. But if there is a need to study the wind effects on specific components or architectural features, then a large-scale study will be required which subsequently introduces the problem of deficient turbulence intensity. The application of the PTS method can be helpful to resolve this issue; however, the scaling parameters should be carefully examined to ensure the applicability of the method.

For instance, the following table (Table 5) shows the scaling parameters for a set of experiments at WOW that were conducted to study the effects of wind loads on the balcony glass handrails of a mid-rise building of $55 \mathrm{~m}$ height. Aerodynamic tests were done at three model scales and two different wind speeds. ASCE7-10 and ASCE49-12 recommend the ${ }^{\mathrm{x}} \mathrm{L}_{\mathrm{u}} / \mathrm{H}\left({ }^{\mathrm{x}} \mathrm{L}_{\mathrm{u}} / \mathrm{B}\right)$ ratio to be similar between model and prototype or greater than three. This condition was satisfied for the 1:67 and 1:180, as shown in Table 5. But considering the applicability of quasi-steady theory, and hence PTS, the ${ }^{x} \mathrm{~L}_{\mathrm{u}} / \mathrm{H}$ has been proposed to be greater than 0.6 while the 1:25 case had a smaller value. Unfortunately, there is no fullscale data available for tall buildings to be used as a benchmark to validate the results. However, since the $1: 67$ scale satisfies the ${ }^{x} L_{u} / H$ scaling requirements and has relatively a high Reynolds number, it can be considered as the benchmark model among the three cases. Since the 1:67 and 1:25 have Reynolds numbers of the same order of magnitude and a blockage ratio under $10 \%$, then any difference in the obtained results from these two models can be mainly attributed to the difference in the ${ }^{x} \mathrm{~L}_{\mathrm{u}} / \mathrm{H}$ proportion. 
Table 5 - Scaling parameters for the models of building with balcony

\begin{tabular}{|l|c|c|c|c|}
\cline { 2 - 5 } \multicolumn{1}{c|}{} & \multicolumn{4}{c|}{ SCALE } \\
\cline { 2 - 5 } \multicolumn{1}{c|}{} & $1: 180$ & $1: 67$ & $1: 25$ & Full-Scale \\
\hline $\mathrm{H}(\mathrm{m})$ & 0.31 & 0.82 & 2.21 & 55.17 \\
\hline $\mathrm{B}(\mathrm{m})$ & 0.14 & 0.36 & 0.98 & 24.38 \\
\hline${ }^{\mathrm{x}} \mathrm{L}_{\mathrm{u}} / \mathrm{H}=$ & 1.31 & 0.61 & 0.23 & 0.51 \\
\hline${ }^{\mathrm{x}} \mathrm{L}_{\mathrm{u}} / \mathrm{B}=$ & 2.95 & 1.37 & 0.51 & 1.15 \\
\hline $\mathrm{U}(\mathrm{mps})$ & 18.3 & 20.7 & 24 & 73.5 \\
\hline $\mathrm{Re}=$ & $1.66 \mathrm{E}+05$ & $1.14 \mathrm{E}+06$ & $3.55 \mathrm{E}+06$ & $2.72 \mathrm{E}+08$ \\
\hline
\end{tabular}

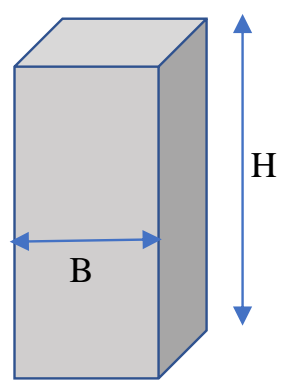




\section{VITA}

\section{MOHAMMADTAGHI MORAVEJ}

\section{EDUCATION \& PROFESSIONAL EXPERIENCE}

- Ph.D. Student \& Research Assistant, Florida Int'l University (2013 - 2018).

- Licensed supervising engineer for construction projects/ Designing buildings (20112013)

- $\quad$ Lecturer at Marvdasht Azad University \& Pishtazan Higher Ed Inst. (2006-2013)

- Master of Science: Structural Engineering, Shiraz University, Iran (2005-2009).

- Computer software instructor, Iran (2000-2013)

- Bachelor of Science: Civil Engineering, Najafabad Azad University, Iran (1999-2004).

\section{PUBLICATIONS \& PRESENTATIONS}

Moravej, M., Irwin, P., Zisis, I., Chowdhury, A.G., Hajra, B., 2017. Effects of roof height on local pressure and velocity coefficients on building roofs. Engineering Structures 150, 693710.

Arch, G., Hajra, B., Moravej, M., Zisis, I., Irwin, P., Chowdhury, A.G., Suaris, W., 2017. An experimental study to assess the effect of soffit louvered vents on wind loads and wind driven rain intrusion on low rise buildings. Sustainable Cities and Society 34, 43-55.

Gan Chowdhury, A., Zisis, I., Irwin, P., Bitsuamlak, G., Pinelli, J.-P., Hajra, B., Moravej, M., 2017. Large-Scale Experimentation Using the 12-Fan Wall of Wind to Assess and Mitigate Hurricane Wind and Rain Impacts on Buildings and Infrastructure Systems. Journal of Structural Engineering 143.

Ludena, L., Mooneghi, M.A., Moravej, M., Chowdhury, A.G., Irwin, P. Estimation of Wind Loads on the Balcony Glass Handrails of Mid-Rise Buildings, in: Structures Congress 2017. pp. 232-243.

Moravej, M., Zisis, I., Chowdhury, A.G., Irwin, P., Hajra, B., 2016. Experimental Assessment of Wind Loads on Vinyl Wall Siding. Frontiers in Built Environment, 2, 35. doi:10.3389/fbuil.2016.00035.

Moravej, M., Gan Chowdhury, A., Zisis, I., Irwin, P., Hajra, B., 2016. The effect of a shutter on the wind induced loads on a window and wind driven rain intrusion into the building through experiments at the Wall of Wind Experimental Facility. Proceedings of 8th Int'l Colloq. on Bluff Body Aerodynamics and Applications, Northeastern University, Boston.

Chowdhury, A.G., Moravej, M., Habte, F., 2016. Wall of Wind Research and Testing to Enhance Resilience of Civil Infrastructure to Hurricane Multi-Hazards, in: Multi-Hazard Approaches to Civil Infrastructure Engineering. Springer, pp. 357-379. [book chapter]

Chowdhury, A.G., Tremente, A., Moravej, M., Hajra, B., Zisis, I., Irwin, P., 2016. Active Aerodynamic Mitigation on a Building Roof, in: The Sixth US-Japan Workshop on Wind Engineering, May 12-14, Tokyo, Japan. 
Moravej, M., Chowdhury, A.G., Irwin, P.A., Zisis, I., Bitsuamlak, G., 2015. Dynamic Effects of Wind Loading on Photovoltaic Systems. Presented at the 14th. International Conference on Wind Engineering (ICWE14), Porto Alegre, Brazil.

Baheru, T., Habte, F., Moravej, M., Gan Chowdhury, A., 2014. Full-Scale Testing to Evaluate Wind Effects on Residential Tiled Roofs. Presented at the ICBEST, Aachen Germany.

Ranjbaran A., Rousta H., Ranjbaran,M., Hashemi,M., Moravej M., 2013. A necessary modification for the finite element analysis of cracked members detection, construction, and justification, Archive of Applied Mechanics, Springer-Verlag.

Ranjbaran A., Moravej M., Khosravi S., 2008. A New Method For Axial Vibration of Bars, ICASTOR journal of Engineering, Vol.2,No;3.

Moravej, M., Hajra, B., Irwin, P., Zisis, I., Chowdhury, A.G., 2016. An Experimental Investigation on the Effects of Building Height on Velocity Coefficients and Local Wind Pressure on Building Roofs. Presented at the 4th. AAWE Workshop, Miami.

Ludena, L., Chowdhury, A.G., Hajra, B., Moravej, M., Mooneghi, M.A., Irwin, P., Zisis, I., 2016. The effect of Balconies on the Wind Induced Loads on a Fifteen Story Building. Presented at the 4th. AAWE Workshop, Miami.

Arch, G., Moravej, M., Hajra, B., Zisis, I., Chowdhury, A.G., Irwin, P., Suarisa, W., 2016. Assessment of wind loads on gable and hip roof buildings through large scale testing at the Wall of Wind experimental facility. Presented at the 8th. International Colloquium on Bluff Body Aerodynamics and Applications, Boston, MA.

Lessons Learned from the NSF ICORPS project, NSF ICORPS Final workshop, Georgia Tech. (2013)

Dynamic Effects of Wind Loads on Photovoltaic Solar Panels, GSAW 2015, FIU. (2013)

Buckling and Damage Detection of Cracked Columns. 4th. National Congress of Civil Engineering. Tehran University. (2012)

On the Vertical Vibration of High Rise Buildings. 5th. International Conference of Seismology and Earthquake Engineering. International Institute of Seismology and Earthquake Engineering, Tehran. (2012)

Some of the education books:

Moravej, M, (2011), Structural Loads, Kelid pub.

Moravej, M, (2011), Applied MATLAB programming, Kelid pub.

Moravej, M, (2008), Excel for Civil Engineers, Kelid pub. 